\title{
Olive-Shaped Organic Cages: Synthesis and Remarkable Promotion of Hydrazone Condensation through Encapsulation in Water
}

Yan-Yan Xu, Hong-Kun Liu, Ze-Kun Wang, Bo Song, Dan-Wei Zhang, ${ }^{*}$ Hui Wang, Zhiming Li, * Xiaopeng Li, and Zhan-Ting Li*

E-mails: zhangdw@fudan.edu.cn,zmli@fudan.edu.cn, ztli@fudan.edu.cn

\section{Contents}

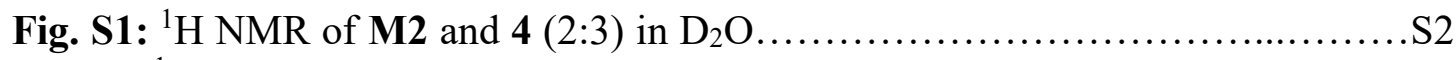

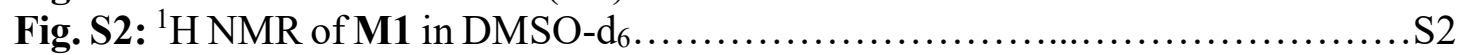

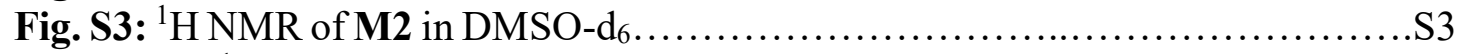

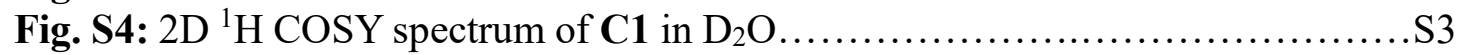

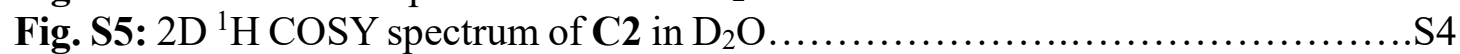

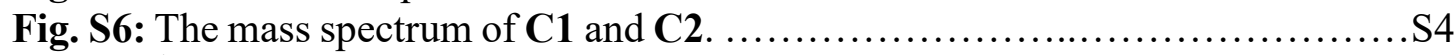

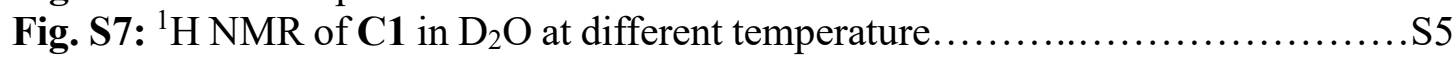

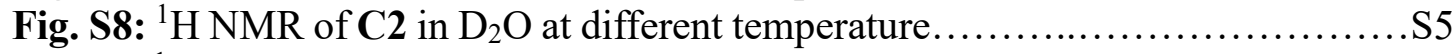

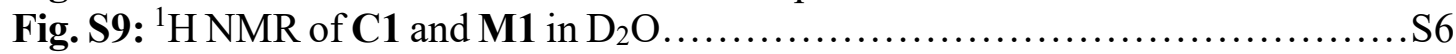

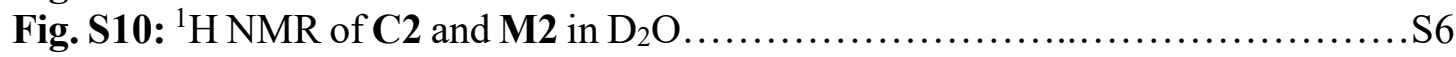

Fig. S11: Optimized low-energy conformations.................................. 7

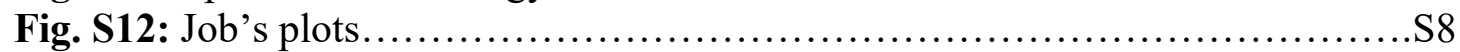

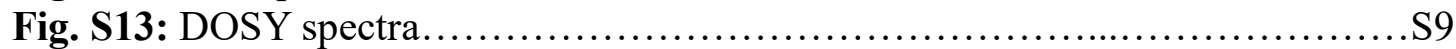

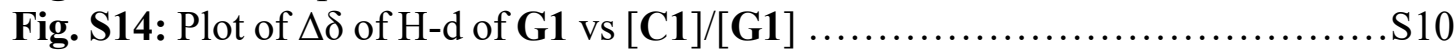

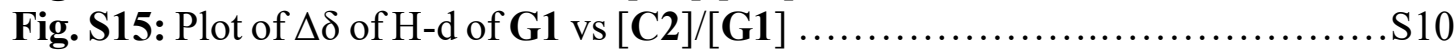

Fig. S16: ${ }^{1} \mathrm{H}$ NMR of C1, G1/C1, G1, C2/G1 and C2 in $\mathrm{D}_{2} \mathrm{O} \ldots \ldots \ldots \ldots \ldots \ldots . . . . . . . . . .11$

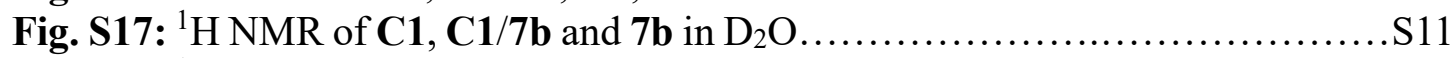

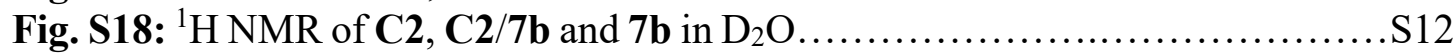

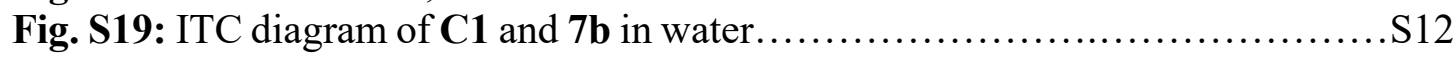

Fig. S20: ITC diagram of $\mathbf{C 2}$ and $\mathbf{7 b}$ in water.................................... 13

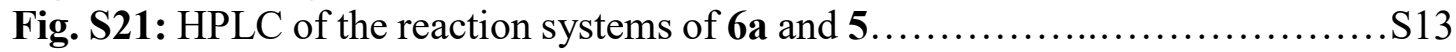

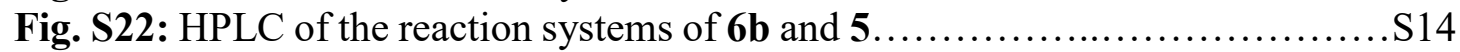

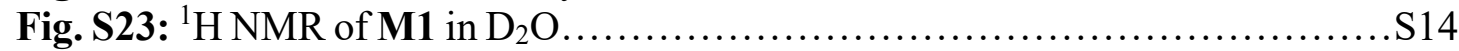

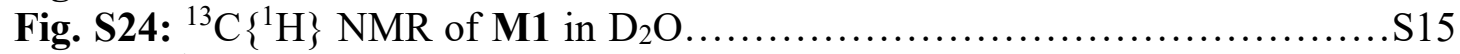

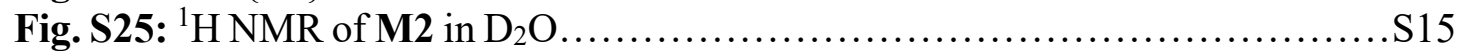

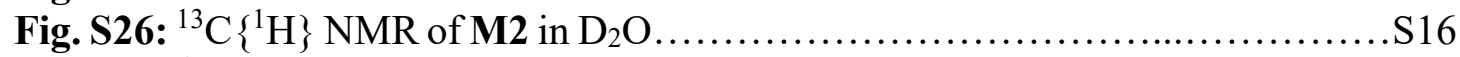

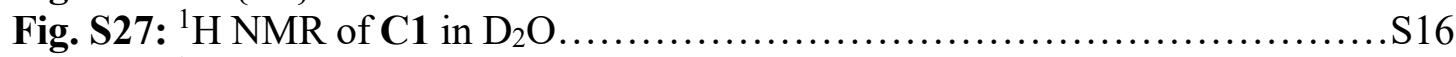

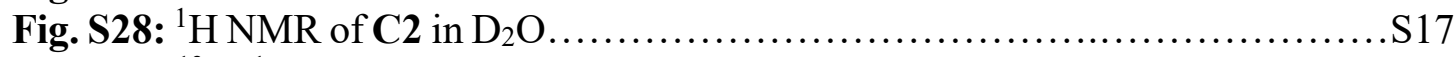

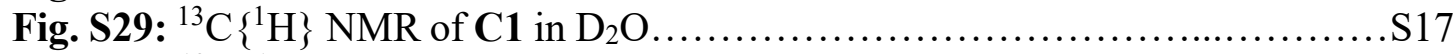

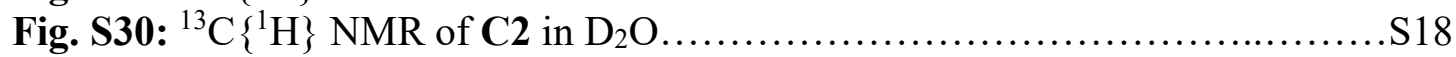

Fig. S31-S54: ${ }^{1} \mathrm{H}$ NMR of 7a, 7b, 9a-9k and 10a-10k in $\mathrm{D}_{2} \mathrm{O} \ldots \ldots \ldots \ldots \ldots . . . . . . . .518-\mathrm{S} 30$

Table S1. Electronic energies and coordinates of the optimized structures.......S31-S46

Table S2. The solubility of Products with or without the Cages in Water.............S47 


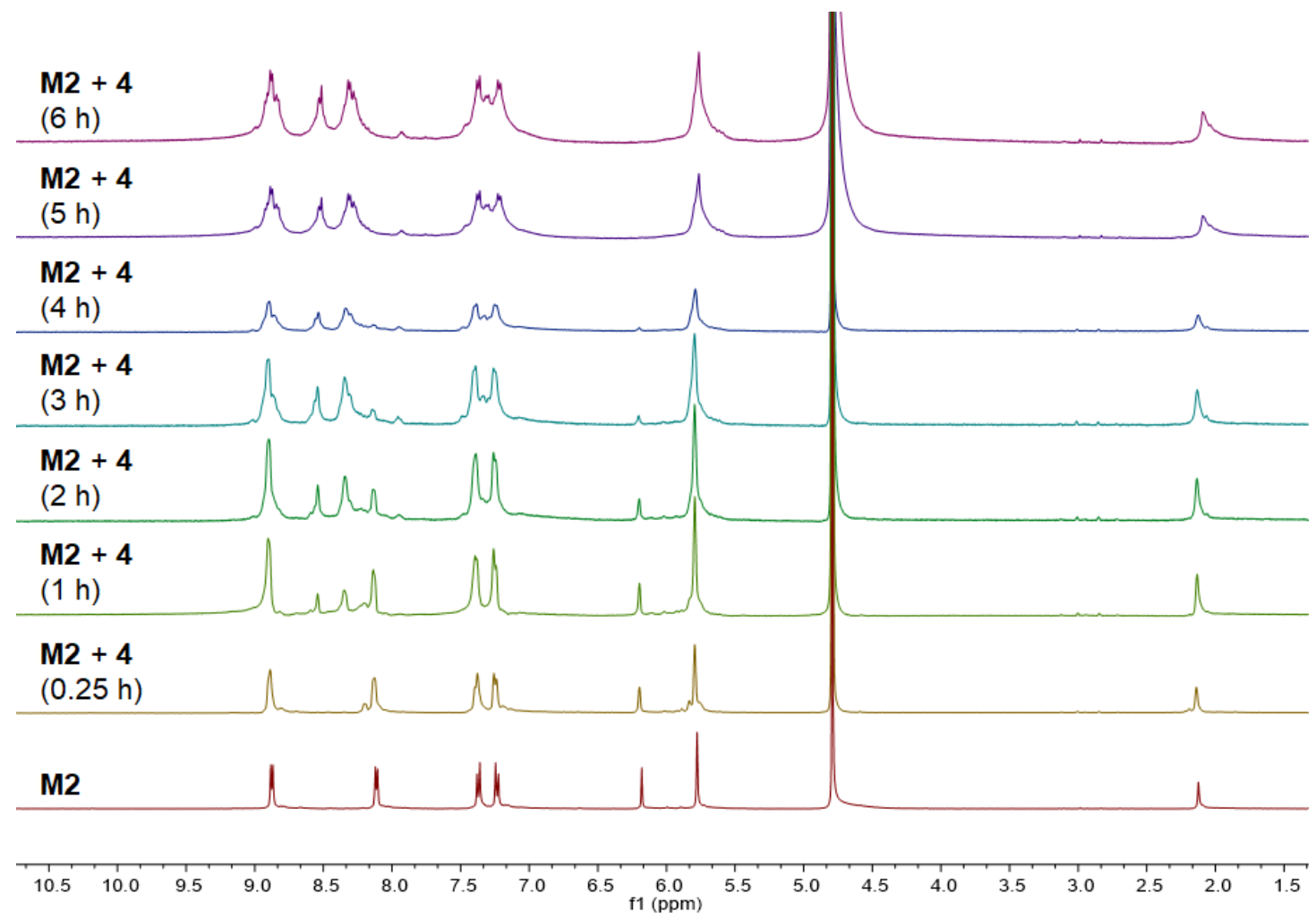

Figure S1. ${ }^{1} \mathrm{H}$ NMR spectrum (400 MHz) of the 2:3 mixture of M2 $(10 \mathrm{mM})$ and 4 (15 $\mathrm{mM}$ ) in $\mathrm{D}_{2} \mathrm{O}$ at $60{ }^{\circ} \mathrm{C}$ after $15 \mathrm{~min}, 1 \mathrm{~h}, 2 \mathrm{~h}, 3 \mathrm{~h}, 4 \mathrm{~h}, 5 \mathrm{~h}$, and $6 \mathrm{~h}$.

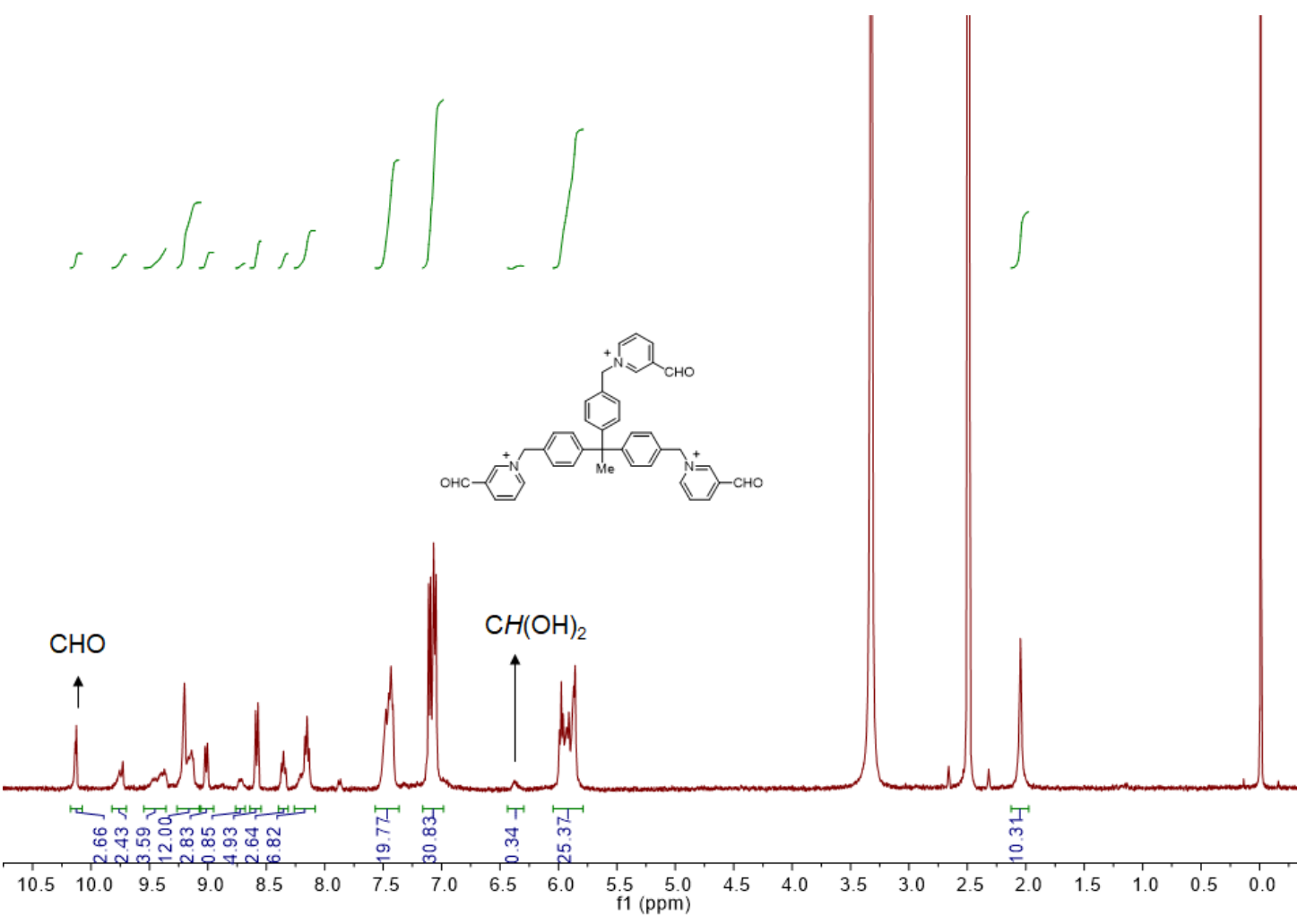

Figure S2. ${ }^{1} \mathrm{H}$ NMR spectrum $(400 \mathrm{MHz})$ of $\mathbf{M 1}$ in DMSO-d ${ }_{6}$ at $25^{\circ} \mathrm{C}$. 


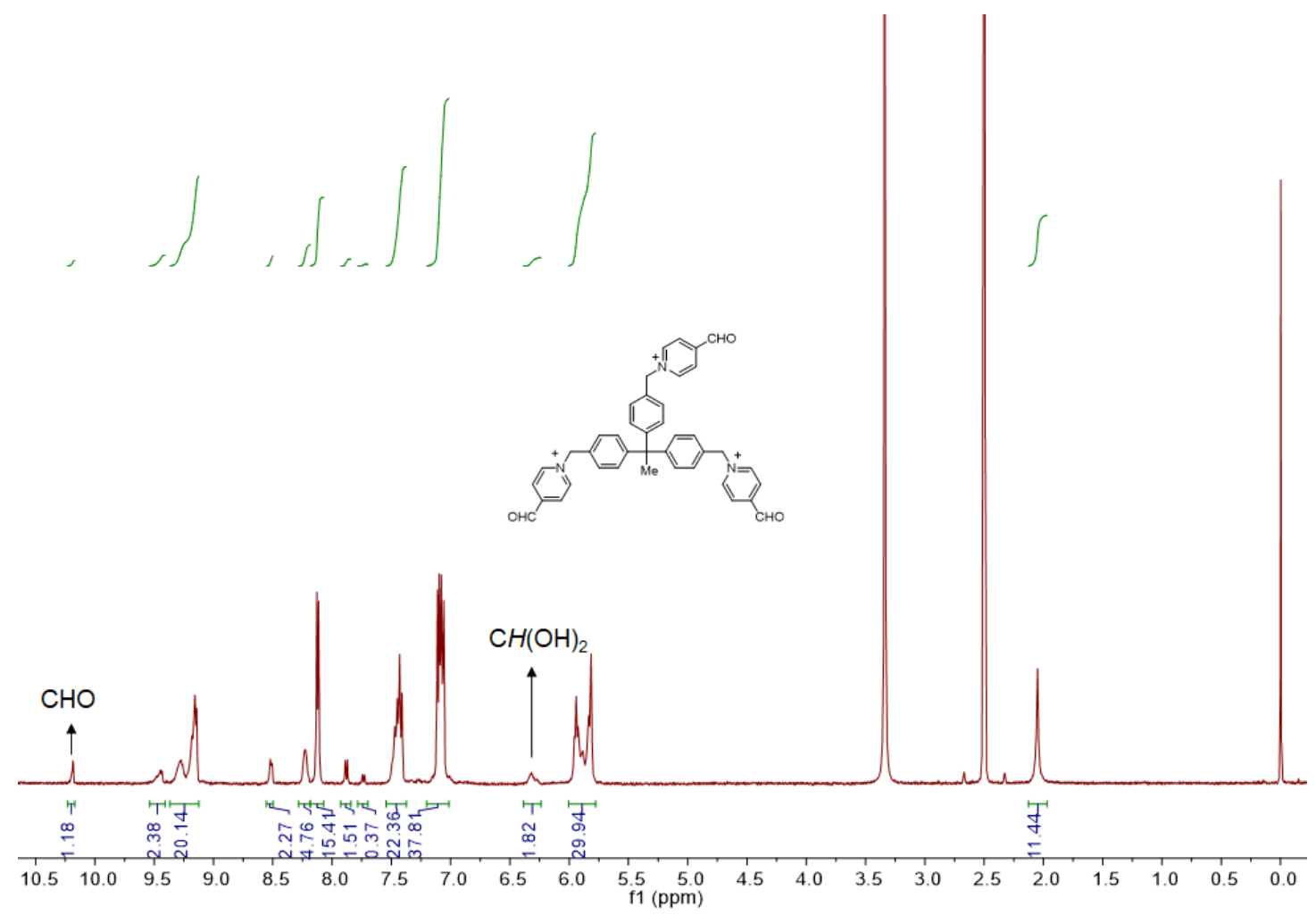

Figure S3. ${ }^{1} \mathrm{H}$ NMR spectrum $(400 \mathrm{MHz})$ of $\mathbf{M} 2$ in DMSO-d 6 at $25^{\circ} \mathrm{C}$.

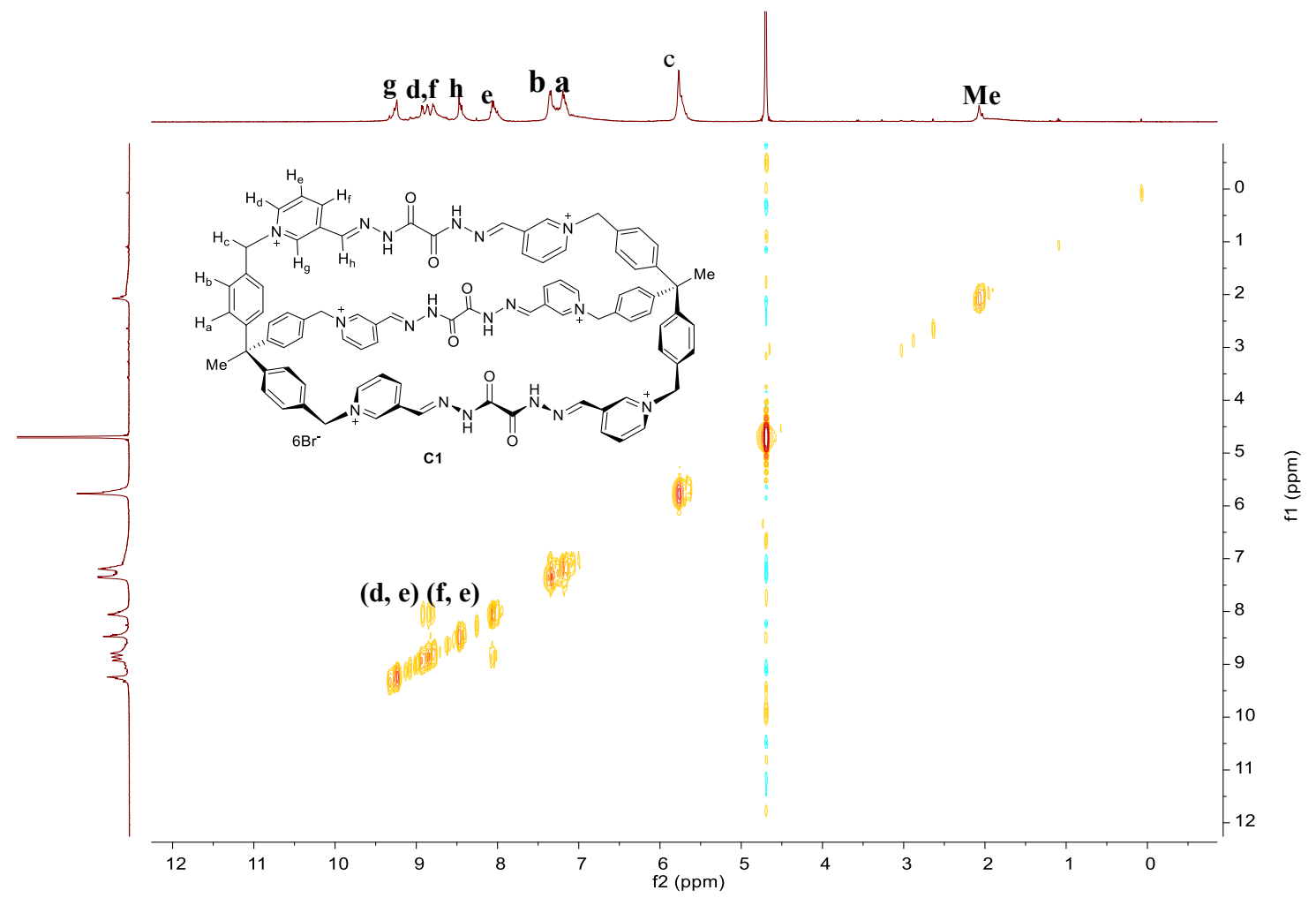

Figure S4. 2D ${ }^{1} \mathrm{H}$ COSY spectrum $(500 \mathrm{MHz})$ of $\mathbf{C 1}$ in $\mathrm{D}_{2} \mathrm{O}$ at $25{ }^{\circ} \mathrm{C}$. 


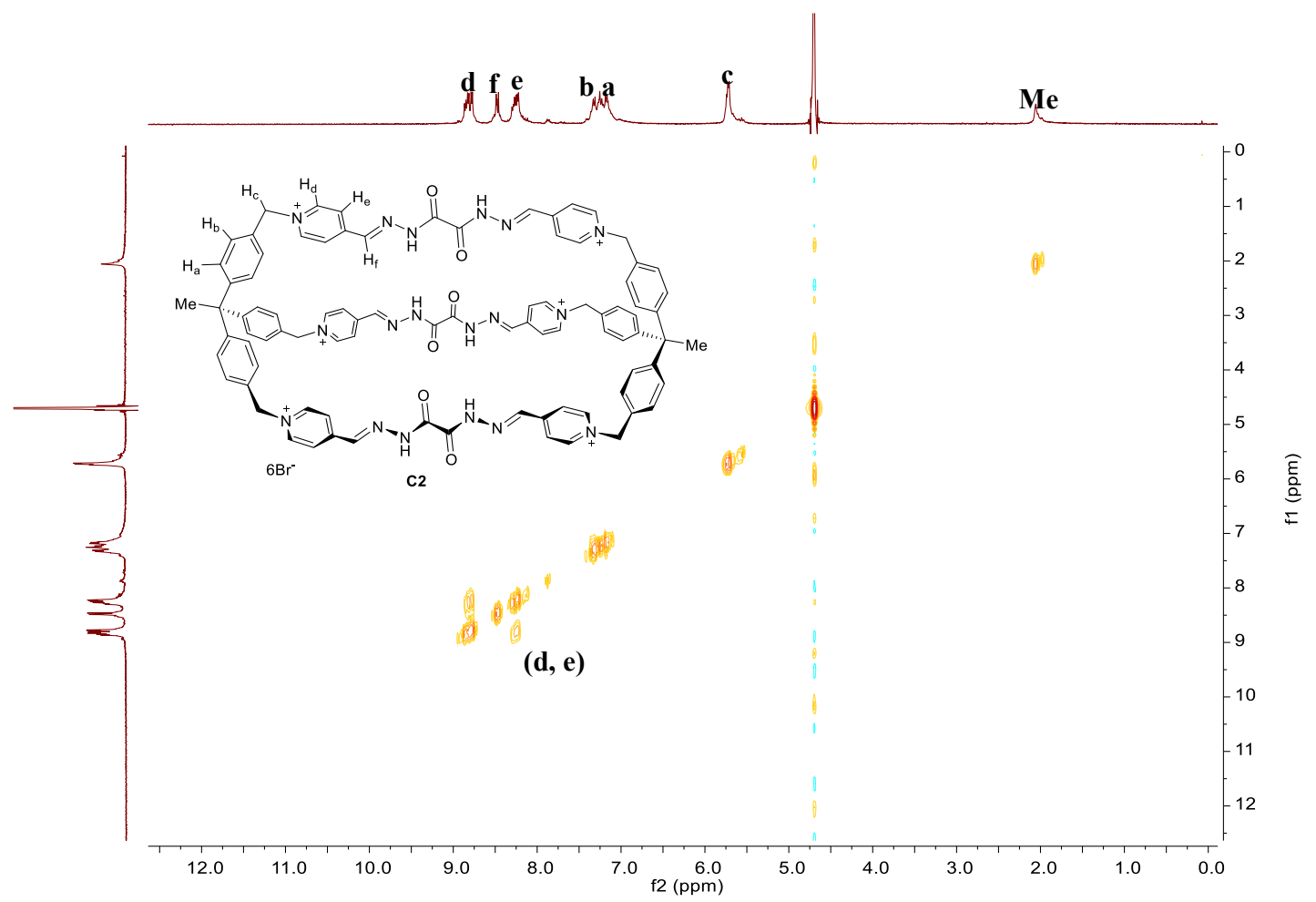

Figure S5. 2D ${ }^{1} \mathrm{H}$ COSY spectrum $(500 \mathrm{MHz})$ of $\mathbf{C 2}$ in $\mathrm{D}_{2} \mathrm{O}$ at $25^{\circ} \mathrm{C}$.

a)

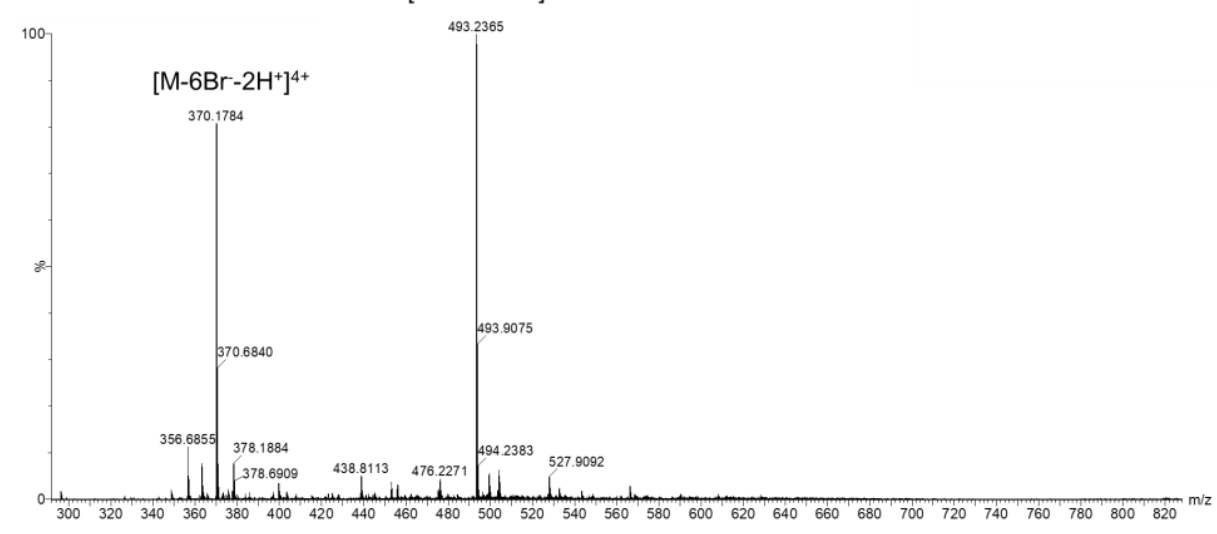

b) $\quad\left[\mathrm{M}+\mathrm{MeOH}-6 \mathrm{Br}-\mathrm{H}^{+}\right]^{5+}$

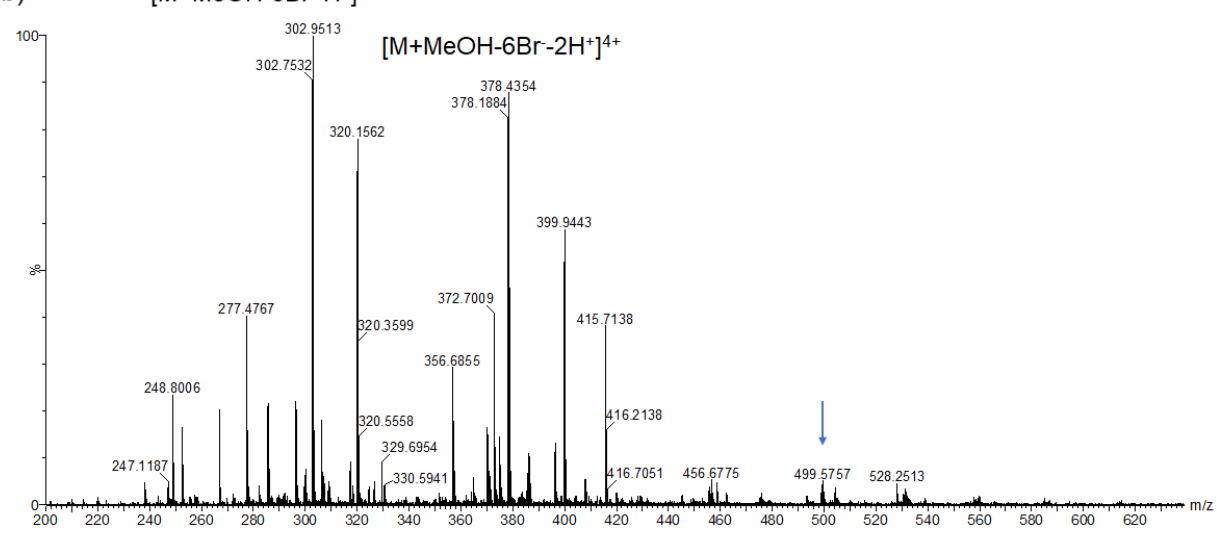

Figure S6. The mass spectrum of a) $\mathbf{C 1}$ and b) $\mathbf{C 2}$. 


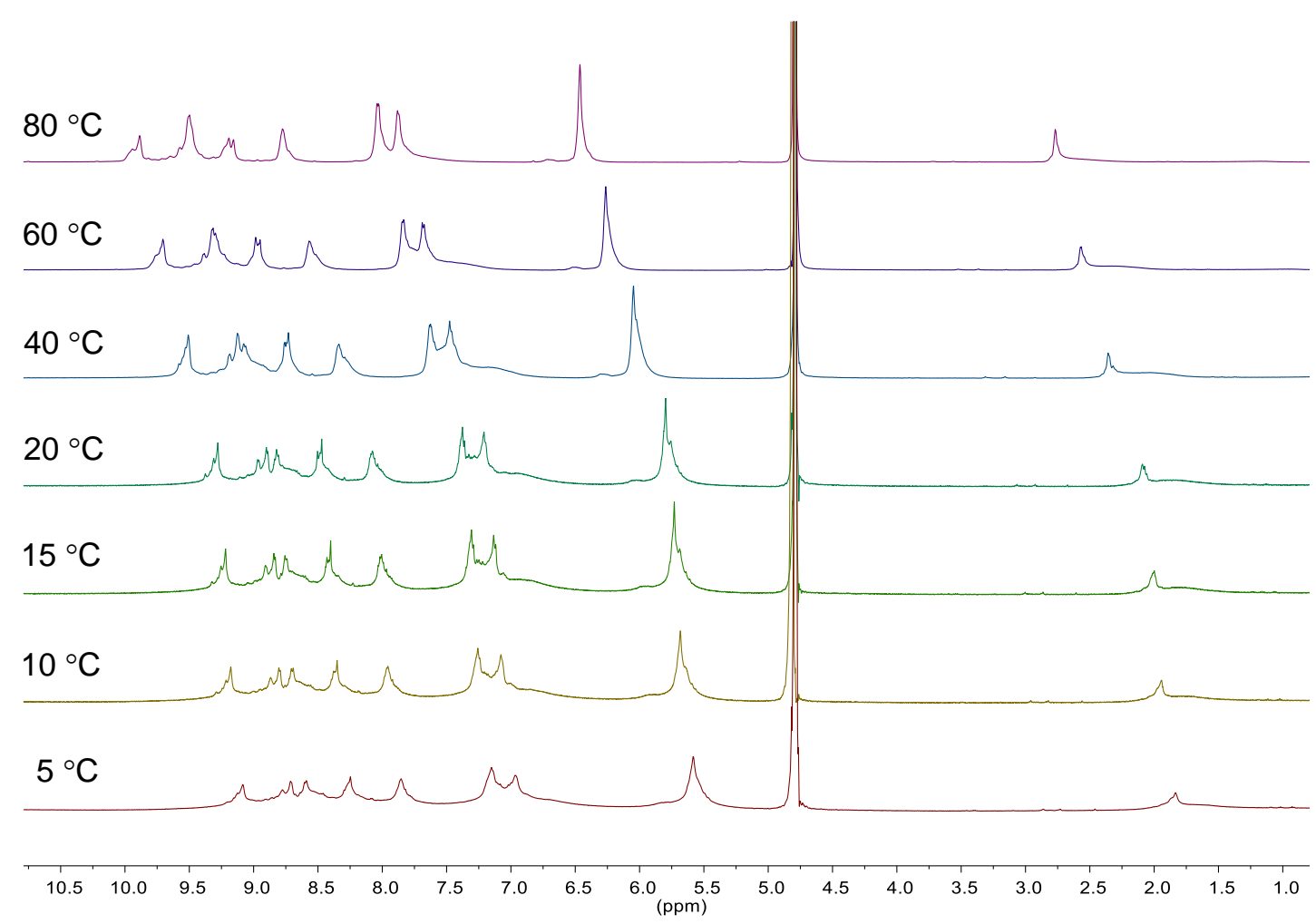

Figure S7. ${ }^{1} \mathrm{H}$ NMR spectrum $(400 \mathrm{MHz})$ of $\mathbf{C 1}$ in $\mathrm{D}_{2} \mathrm{O}$ at different temperature, including $5,10,15,20,40,60$ and $80^{\circ} \mathrm{C}$.

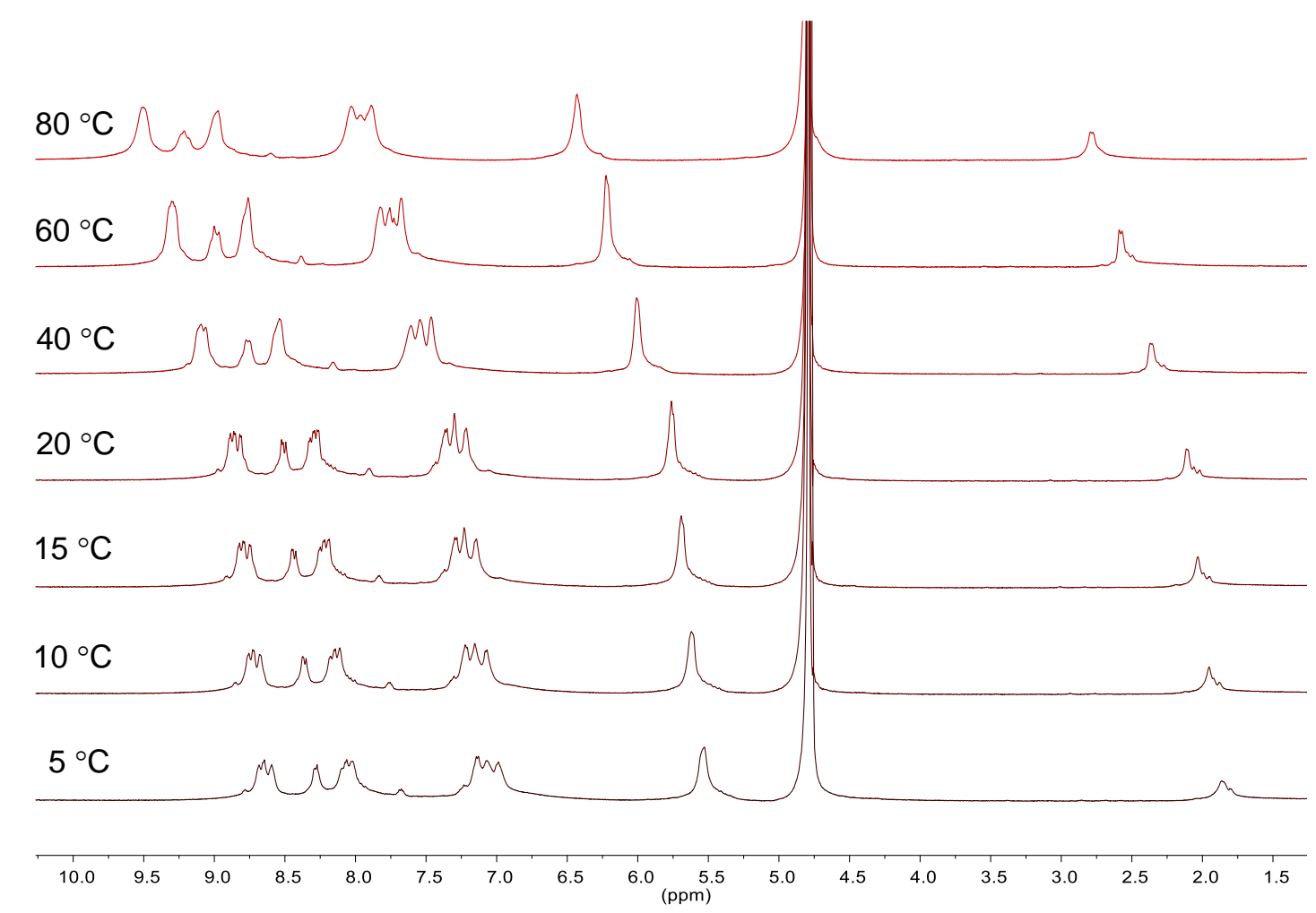

Figure S8. ${ }^{1} \mathrm{H}$ NMR spectrum $(400 \mathrm{MHz})$ of $\mathbf{C 2}$ in $\mathrm{D}_{2} \mathrm{O}$ at different temperature, including $5,10,15,20,40,60$ and $80^{\circ} \mathrm{C}$. 


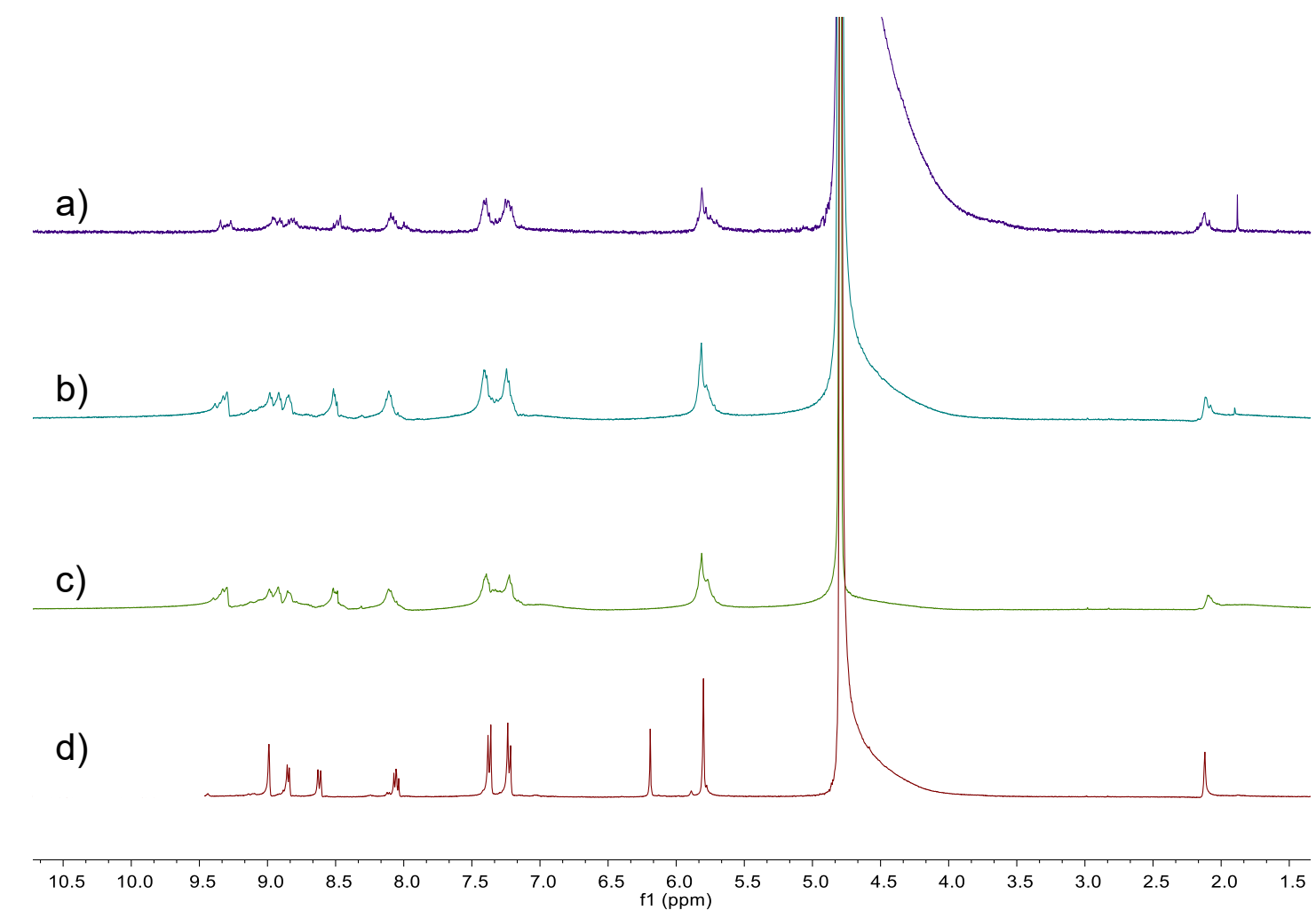

Figure S9. ${ }^{1} \mathrm{H}$ NMR $(400 \mathrm{MHz})$ of a) $\mathbf{C 1}(0.5 \mathrm{mM})$, b) $\mathbf{C 1}(2.5 \mathrm{mM})$, c) $\mathbf{C 1}(5.0 \mathrm{mM})$ and d) $\mathrm{M1}(5.0 \mathrm{mM})$ in $\mathrm{D}_{2} \mathrm{O}$ at $25^{\circ} \mathrm{C}$.

a)

b)

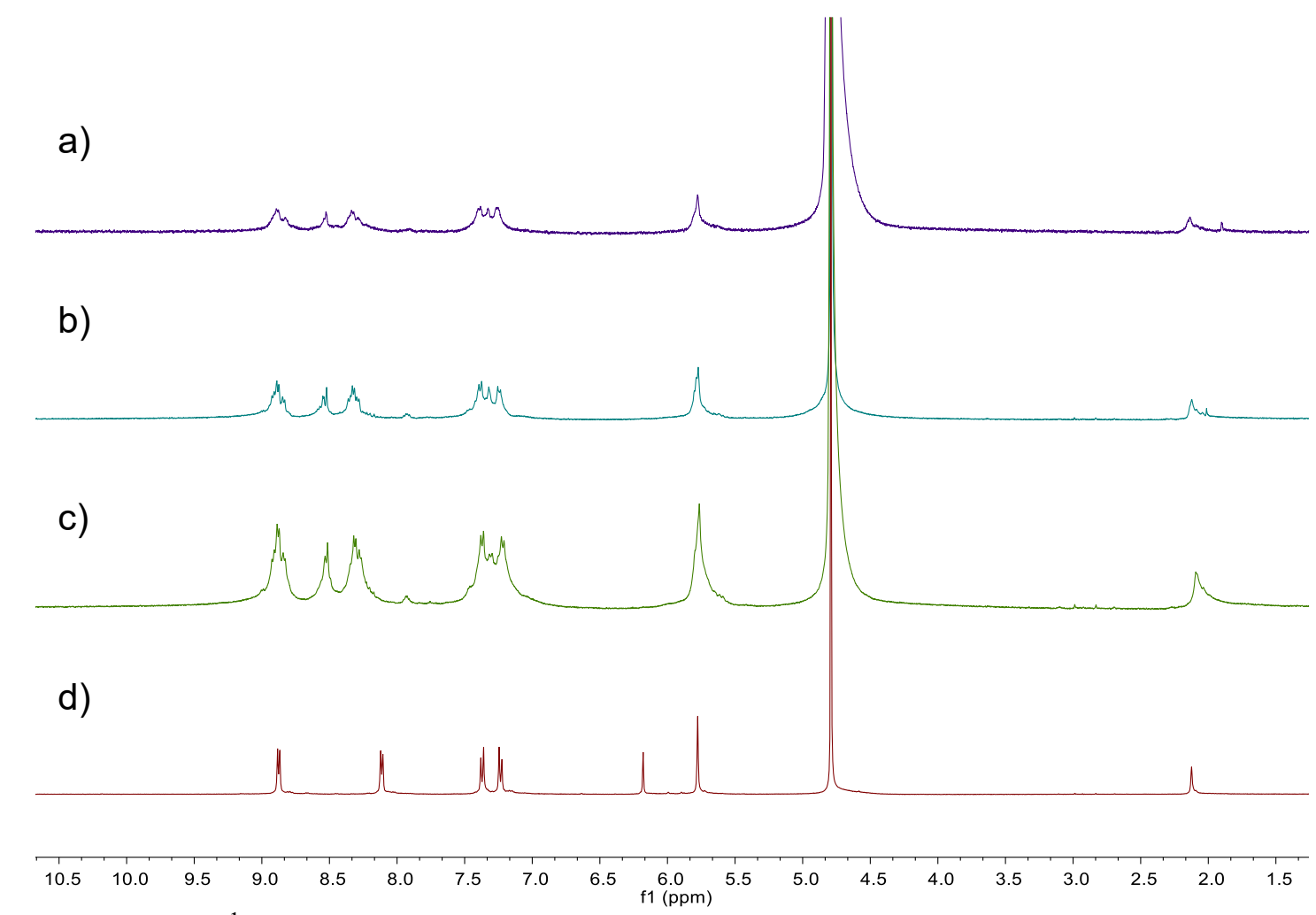

Figure S10. ${ }^{1} \mathrm{H}$ NMR spectrum $(400 \mathrm{MHz})$ of $\mathbf{C 2}(0.5 \mathrm{mM})$, b) $\mathbf{C 2}(2.5 \mathrm{mM})$, c) C2 $(5.0 \mathrm{mM})$ and d) $\mathbf{M} 2(5.0 \mathrm{mM})$ in $\mathrm{D}_{2} \mathrm{O}$ at $25^{\circ} \mathrm{C}$. 


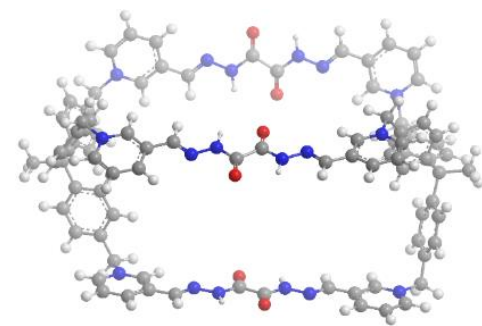

C1-1 (0.0)

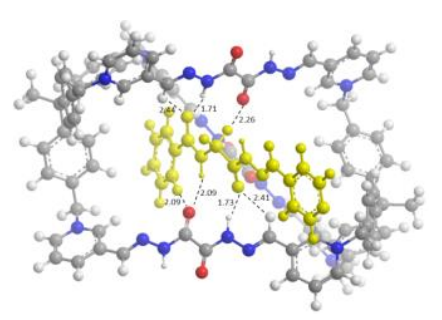

C1 $\subset 7 b-1(0.0)$

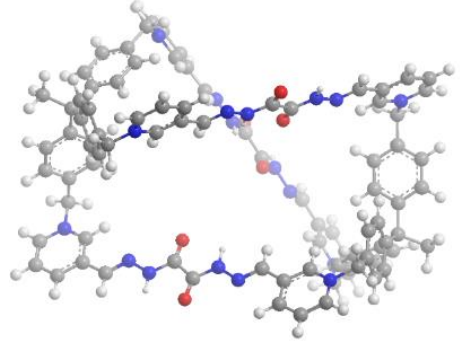

C1-2 (0.5)

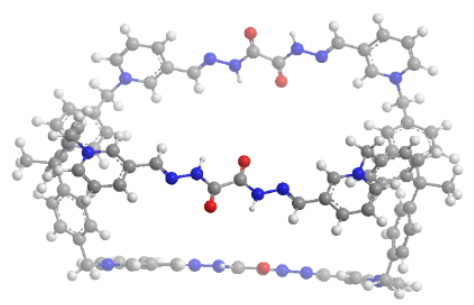

C1-3 (1.2)
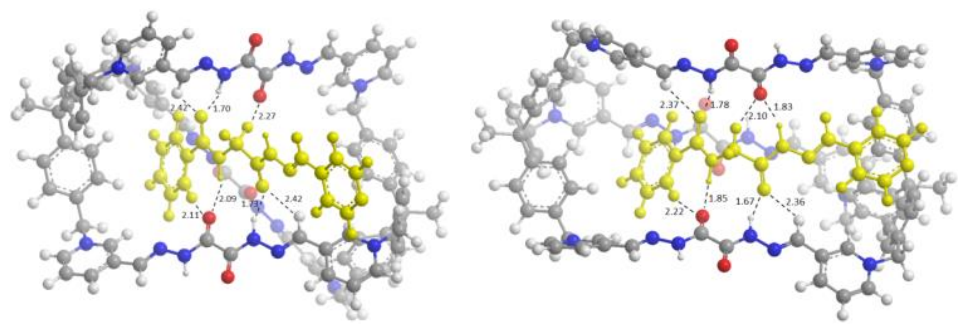

C1 $\subset 7 b-2(0.1)$

C1 $\subset 7 b-3(0.4)$

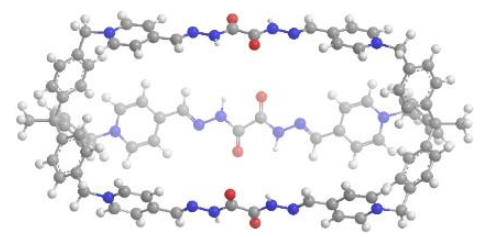

C2-1 (0.0)

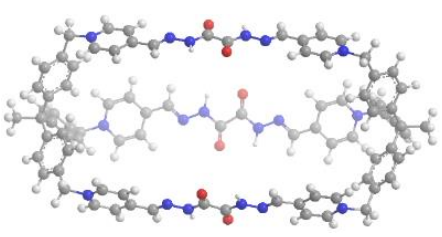

C2-2 (0.0)

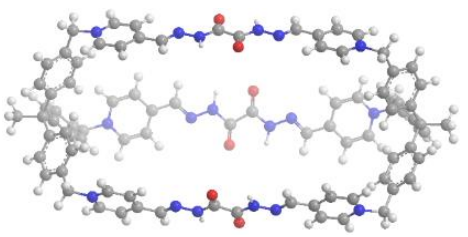

C2-3 (0.0)

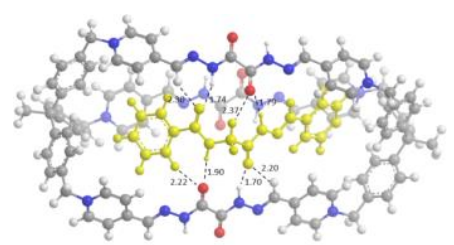

$$
\text { C2 } \subset 7 b-1(0.0)
$$

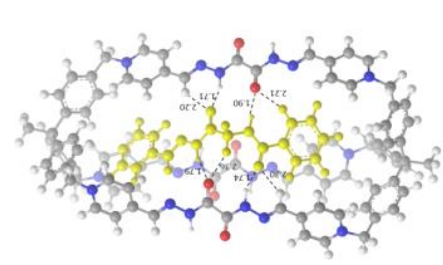

C2 $\subset 7 b-2(0.0)$

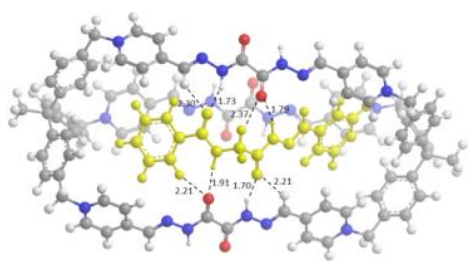

C2 $\subset 7 b-3(0.1)$

Figure S11. The optimized low-energy conformation structures of cage $\mathbf{C 1}$, complex $\mathbf{C 1} \subset \mathbf{7 b}$, cage $\mathbf{C 2}$ and complex $\mathbf{C 2} \subset \mathbf{7 b}$. The values in brackets represent the relative energies in $\mathrm{kcal} / \mathrm{mol}$. 
a) $[\mathbf{G 1}] /([\mathbf{C} 1]+[\mathbf{G} 1])$
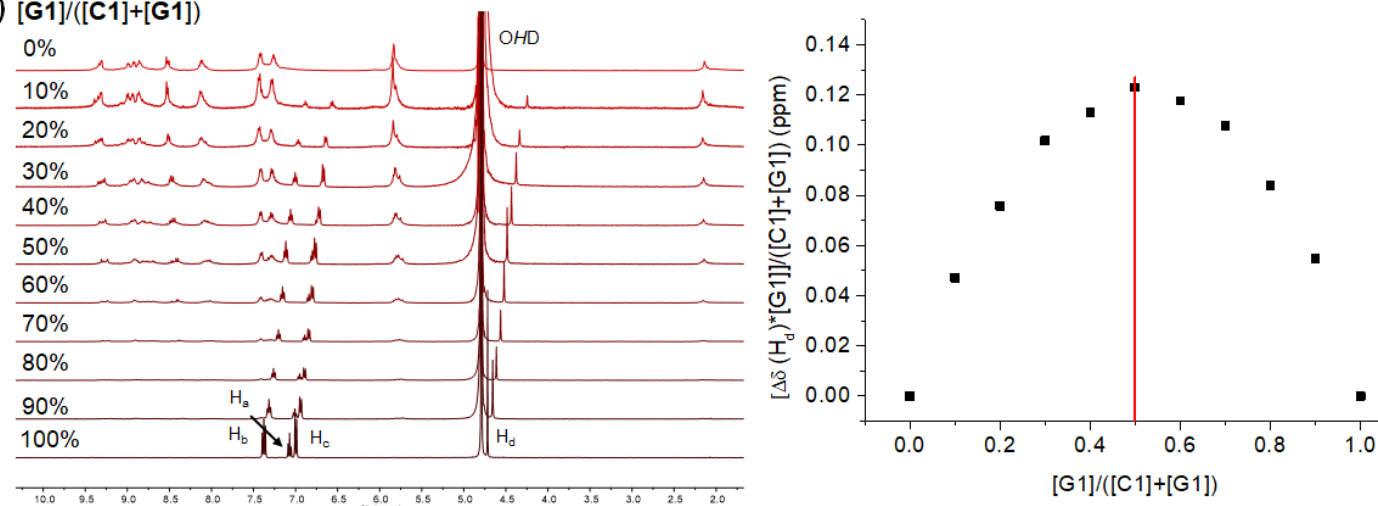

b) $[\mathbf{G} 1] /([\mathbf{C} 2]+[\mathbf{G} 1])$
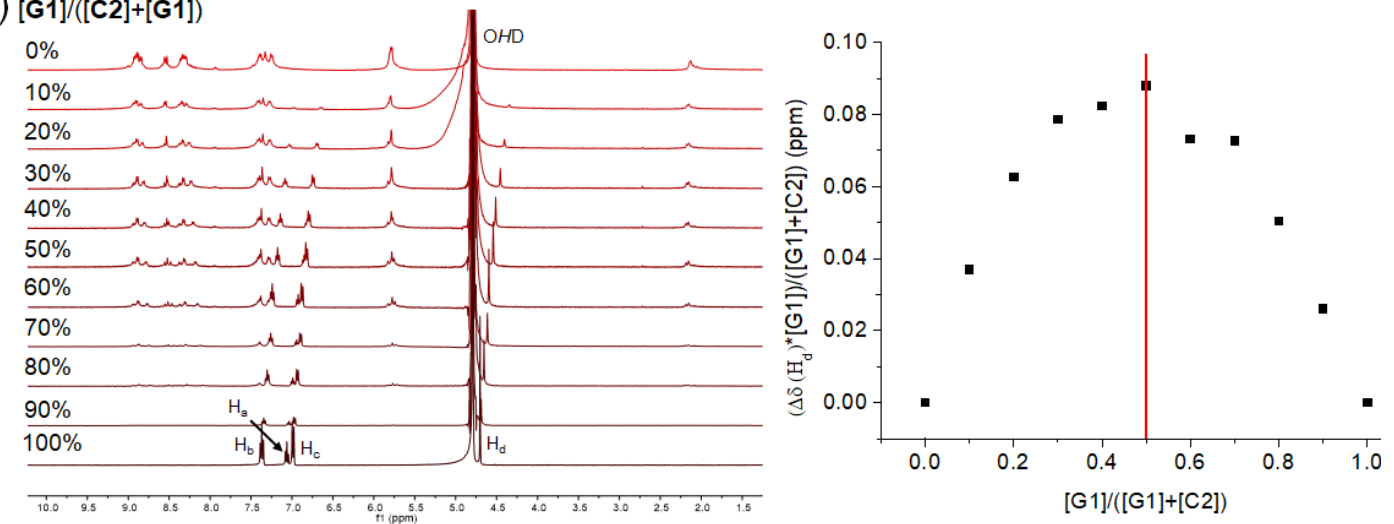

Figure S12. ${ }^{1} \mathrm{H}$ NMR Job's plot for the mixture of a) $\mathbf{C 1}$ and $\mathbf{G 1}$ and b) $\mathbf{C 2}$ and G1 $([\mathbf{C 1}]+[\mathbf{G 1}]=[\mathbf{C 1}]+[\mathbf{G} 2]=2 \mathrm{mM})$. 

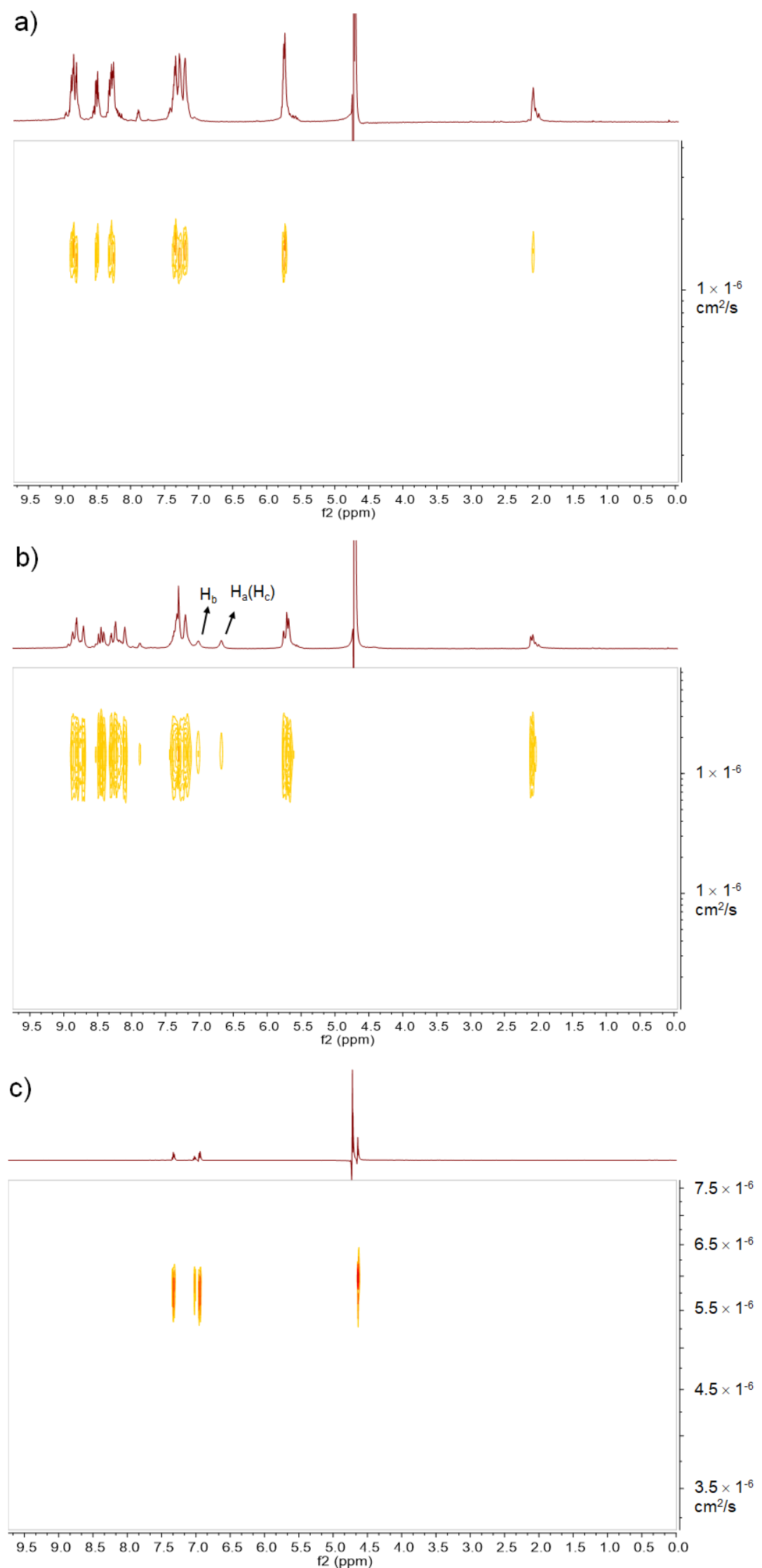

Figure S13. DOSY spectrum (400 MHz) of a) C2, b) $\mathbf{C 2}+\mathbf{G 1}$, and c) $\mathbf{G 1}$ in $\mathrm{D}_{2} \mathrm{O}$. The concentration was $2.5 \mathrm{mM}$ for all the components. 

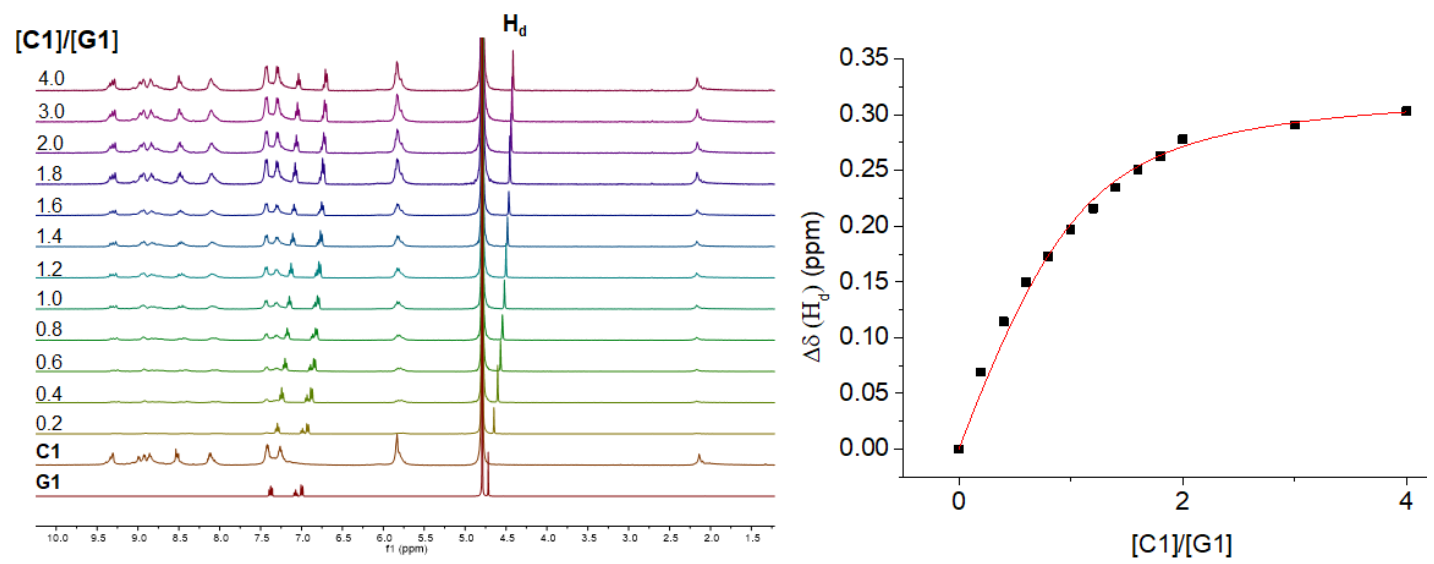

Figure S14. Plot of $\Delta \delta$ of H-d of G1 (see the mail text) versus $[$ C1 $] /[$ G1 $]([\mathbf{G 1}]=2$ $\mathrm{mM})$.
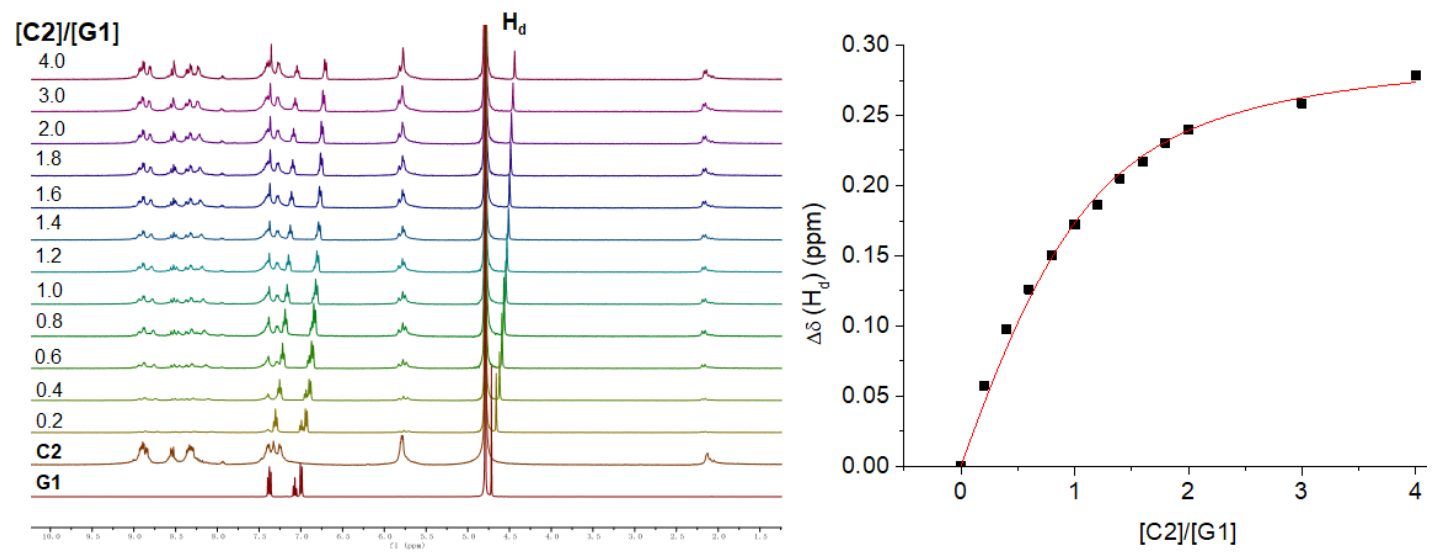

Figure S15. Plot of $\Delta \delta$ of $\mathrm{H}-\mathrm{d}\left(\mathrm{CH}_{2}\right)$ of $\mathbf{G 1}$ versus $[\mathbf{C 2}] /[\mathbf{G 1}]([\mathbf{G 1}]=2 \mathrm{mM})$. 

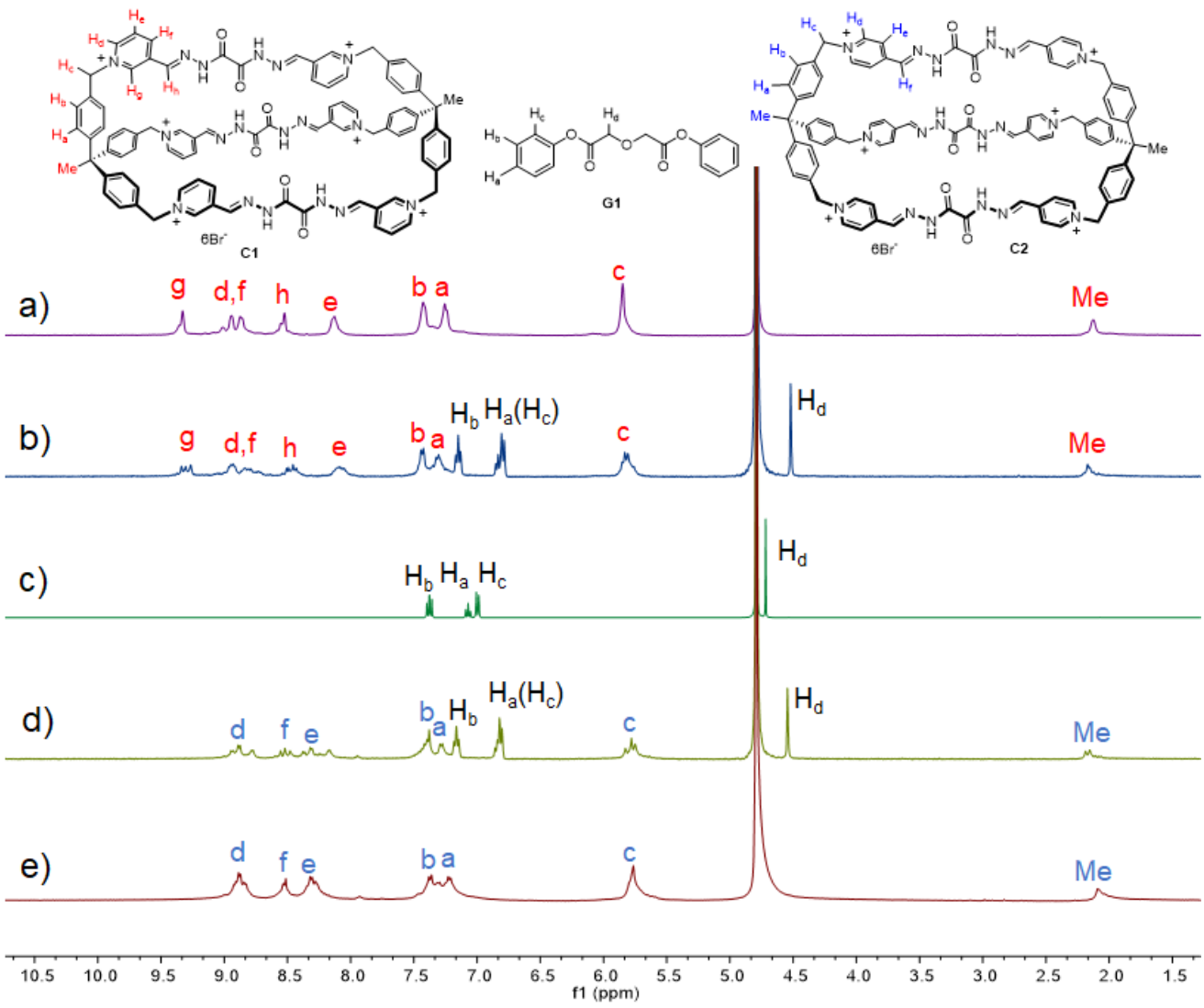

Figure S16. ${ }^{1} \mathrm{H}$ NMR spectrum $(400 \mathrm{MHz})$ of a) C1, b) $\mathbf{G 1}+\mathbf{C 1}$, c) G1, d) $\mathbf{C 2}+\mathbf{G 1}$, e) $\mathbf{C 2}([\mathrm{C} 1]=[\mathrm{C} 2]=[\mathrm{G} 1]=1.25 \mathrm{mM})$ in $\mathrm{D}_{2} \mathrm{O}$ at $25^{\circ} \mathrm{C}$.

a)

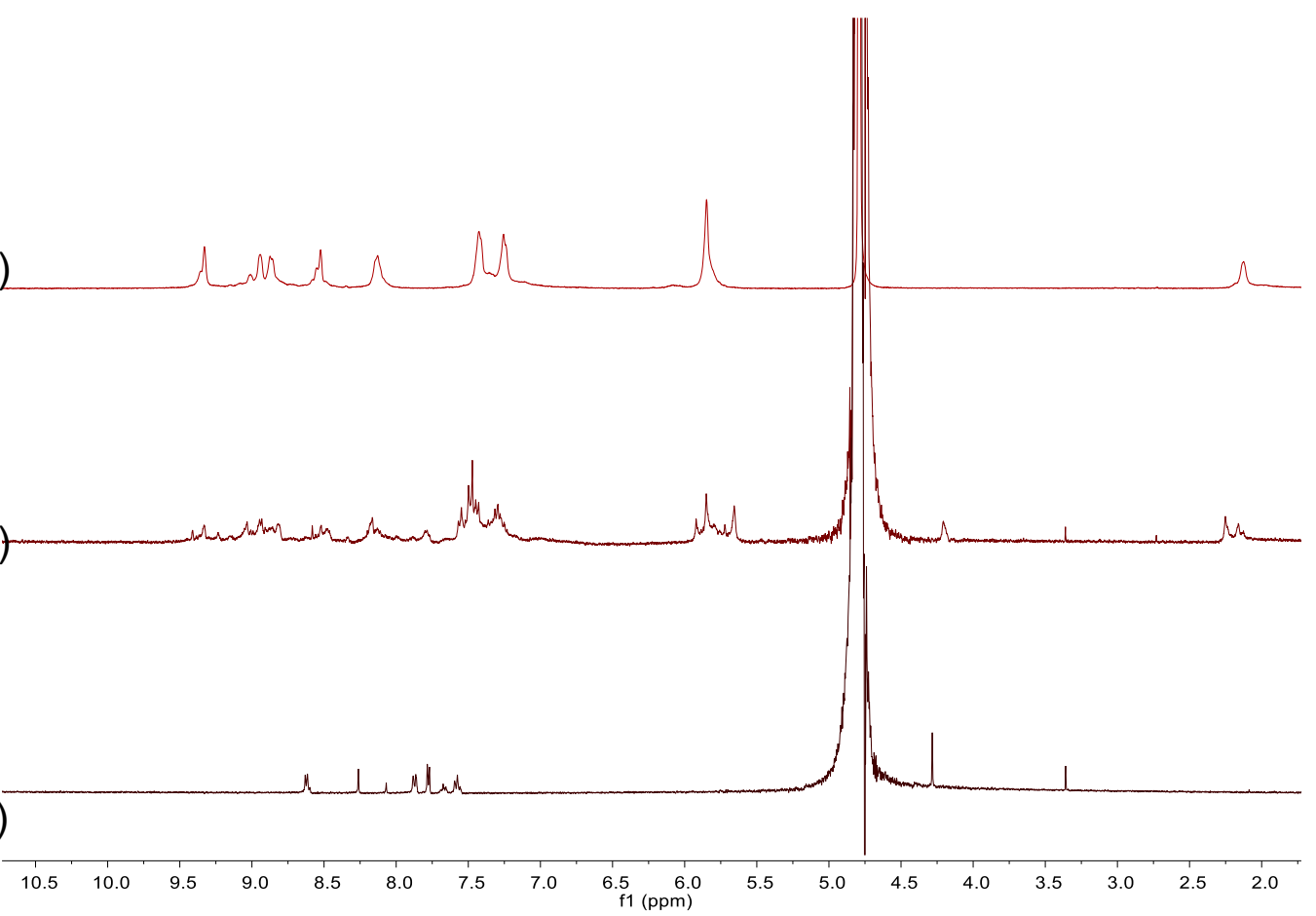

Figure S17. ${ }^{1} \mathrm{H}$ NMR spectrum $(400 \mathrm{MHz})$ of a) $\mathbf{C 1}$, b) $\mathbf{C 1}+\mathbf{7 b}([\mathbf{C 1}]=[\mathbf{7 b}]=0.083$ $\mathrm{mM}$ ) and c) $7 \mathbf{b}$ in $\mathrm{D}_{2} \mathrm{O}$ at $25^{\circ} \mathrm{C}$. 


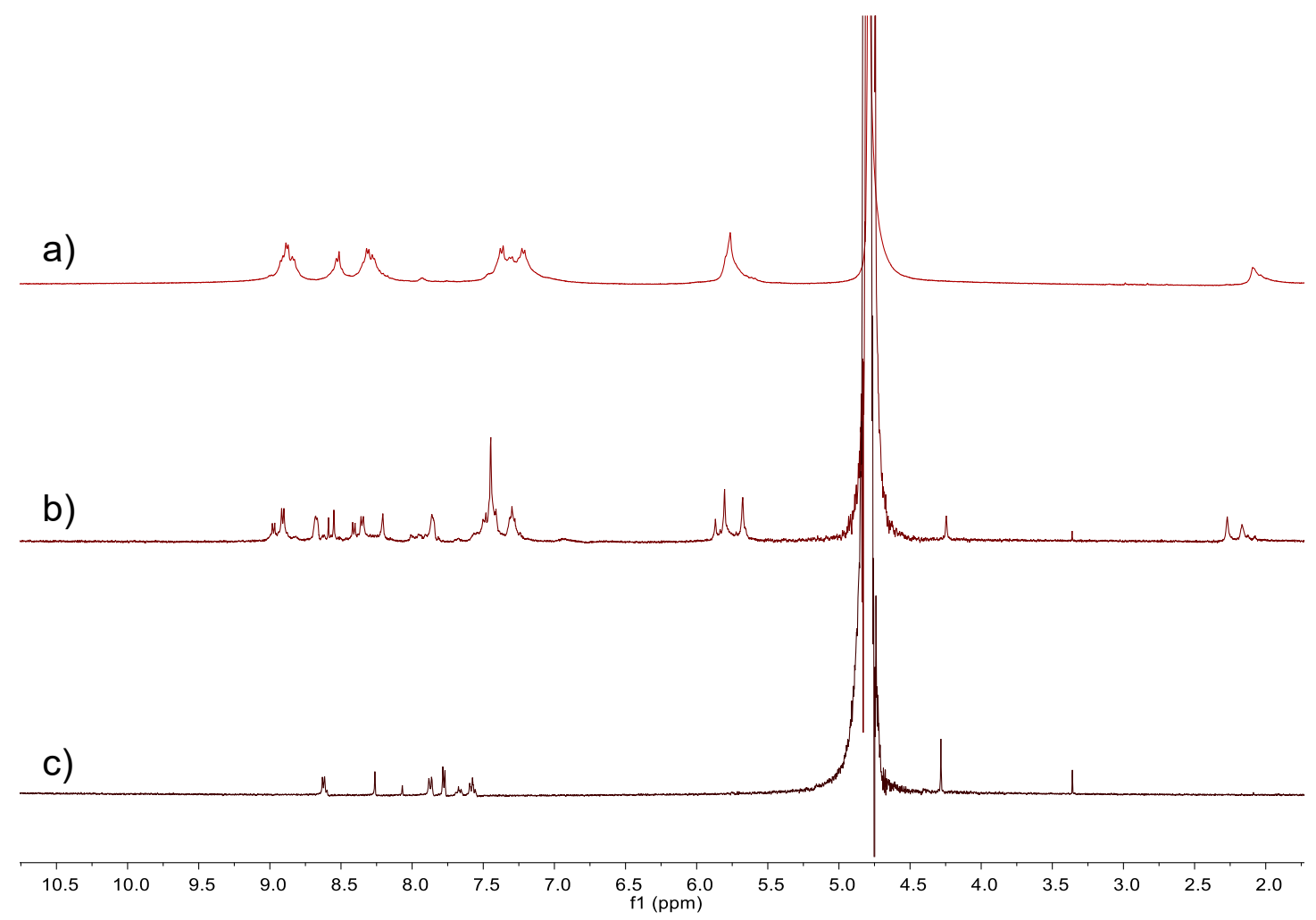

Figure S18. ${ }^{1} \mathrm{H}$ NMR spectrum $(400 \mathrm{MHz})$ of a) $\mathbf{C 2}$, b) $\mathbf{C 2}+\mathbf{7 b}([\mathbf{C 1}]=[7 \mathbf{b}]=0.083$ $\mathrm{mM})$ and c) $7 \mathbf{b}$ in $\mathrm{D}_{2} \mathrm{O}$ at $25^{\circ} \mathrm{C}$.

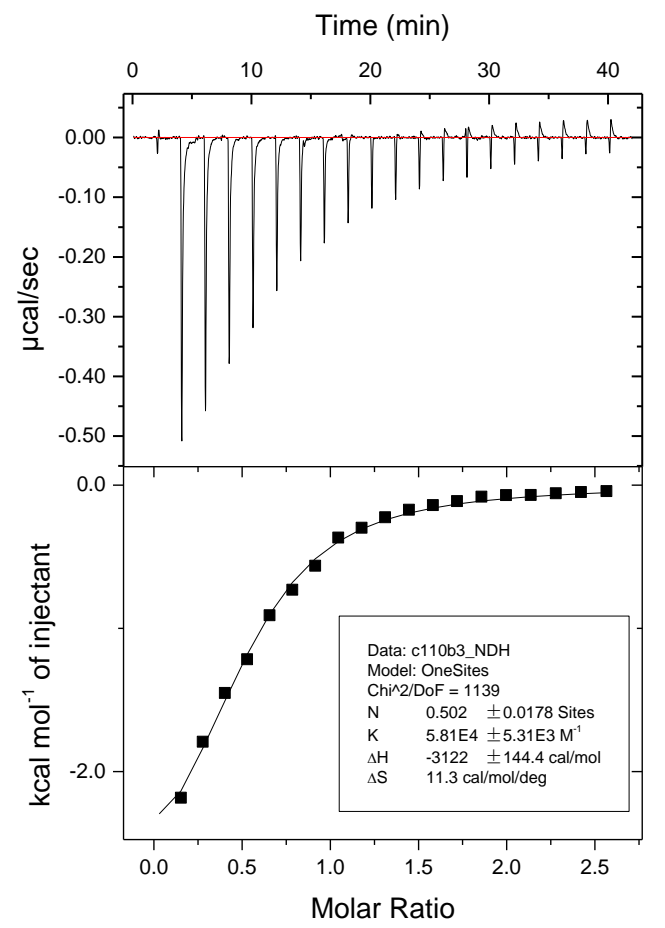

Figure S19. Isothermal titration calorimetric diagram of $\mathbf{C 1}(0.1 \mathrm{mM})$ and $7 \mathbf{b}(1.25$ $\mathrm{mM}$ ) in water at $25^{\circ} \mathrm{C}$. 


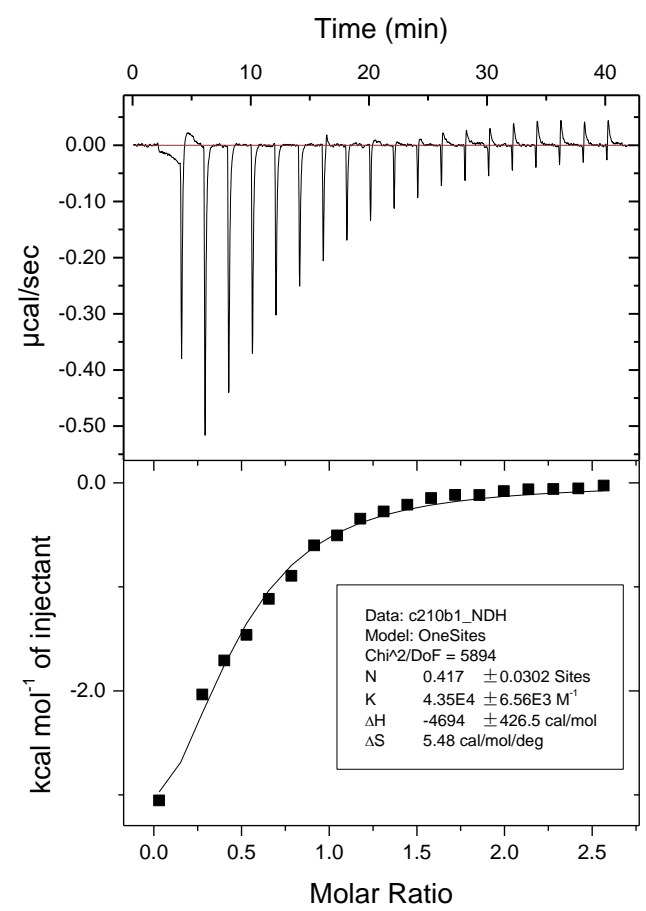

Figure S20. Isothermal titration calorimetric diagram of $\mathbf{C 2}(0.1 \mathrm{mM})$ and $7 \mathbf{b}(1.25$ $\mathrm{mM})$ in water at $25^{\circ} \mathrm{C}$.

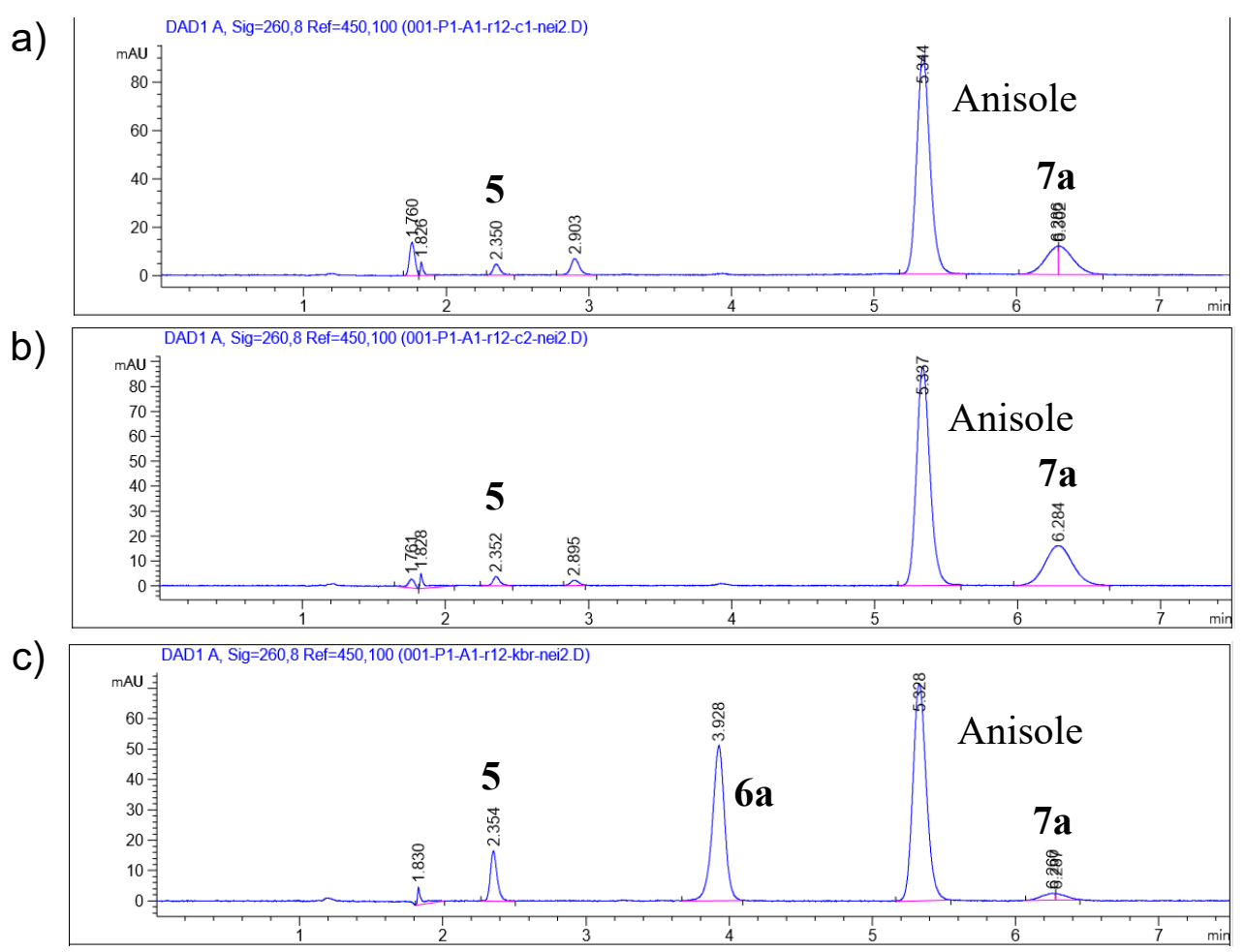

Figure S21. HPLC spectrum of the reaction systems of $\mathbf{6 a}$ and $\mathbf{5}$ in the solution of a) $\mathbf{C 1}$, b) $\mathbf{C 2}$ and c) $\mathbf{K B r}$. 
a)

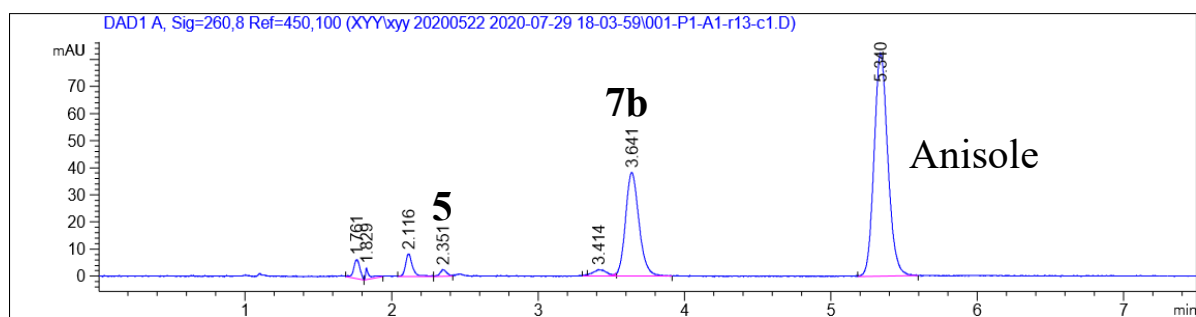

b)

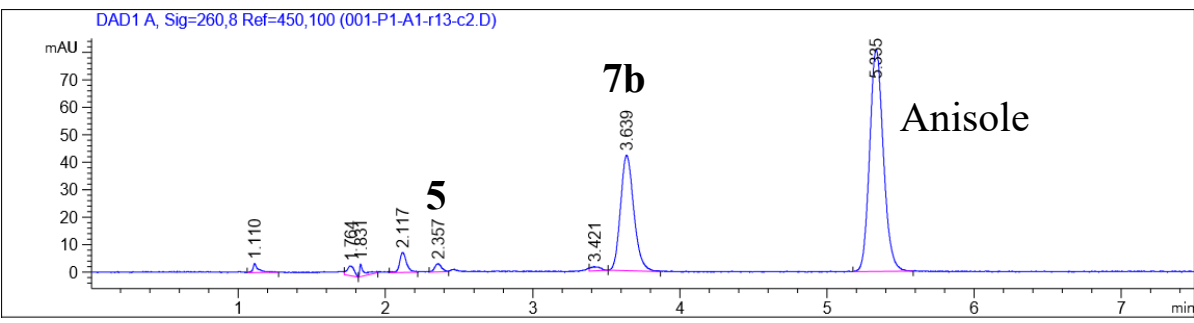

c)

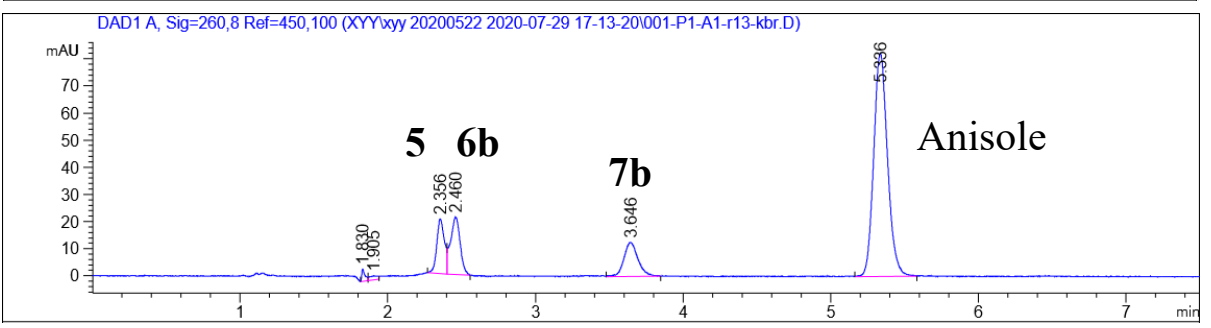

Figure S22. HPLC spectrum of the reaction system of $\mathbf{6 b}$ and $\mathbf{5}$ in the solution of a) C1, b) C2 and c) KBr.

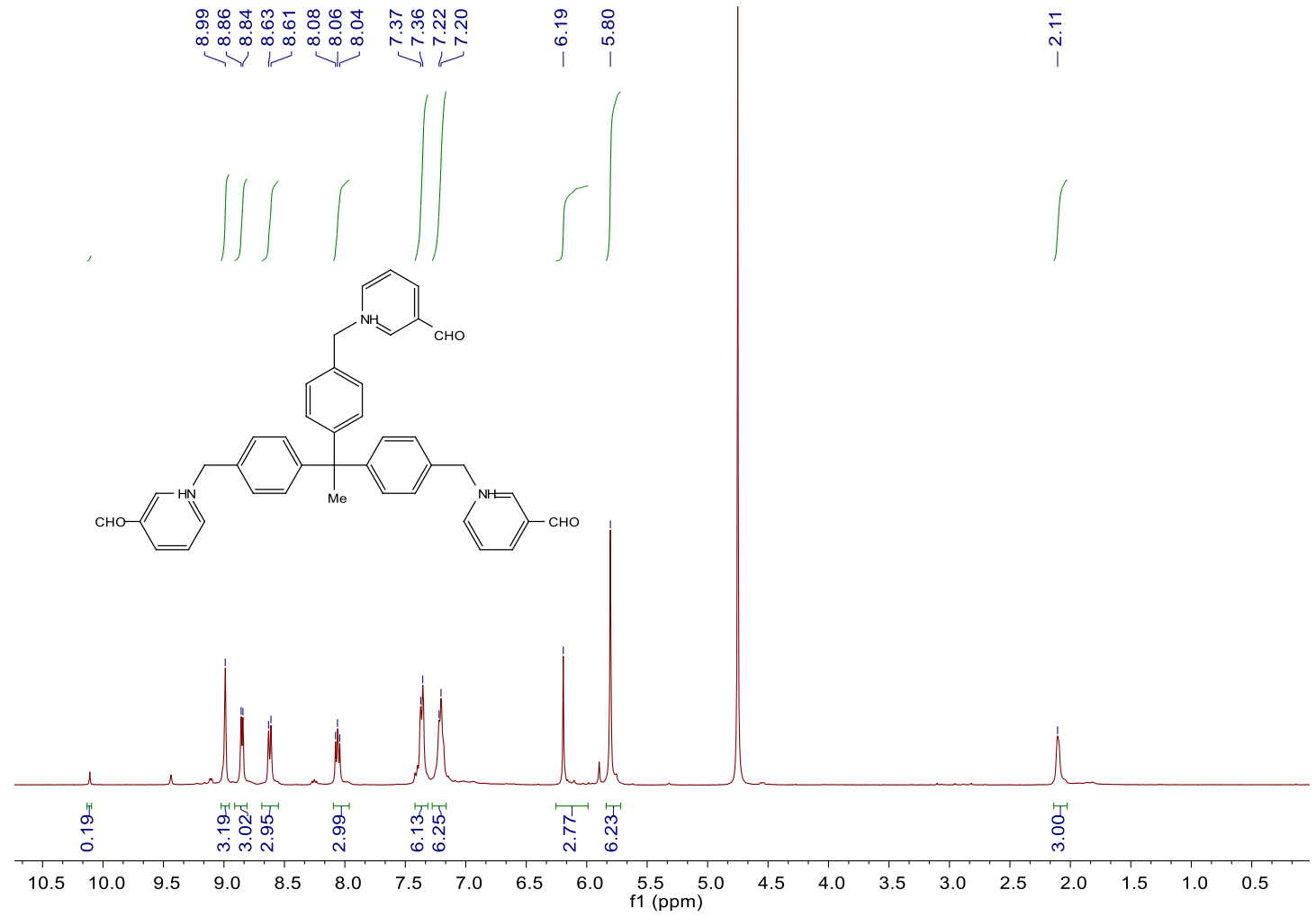

Figure S23. ${ }^{1} \mathrm{H}$ NMR spectrum $(400 \mathrm{MHz})$ of $\mathbf{M 1}$ in $\mathrm{D}_{2} \mathrm{O}$ at $25^{\circ} \mathrm{C} .94 \%$ of the aldehyde groups have been hydrated. 


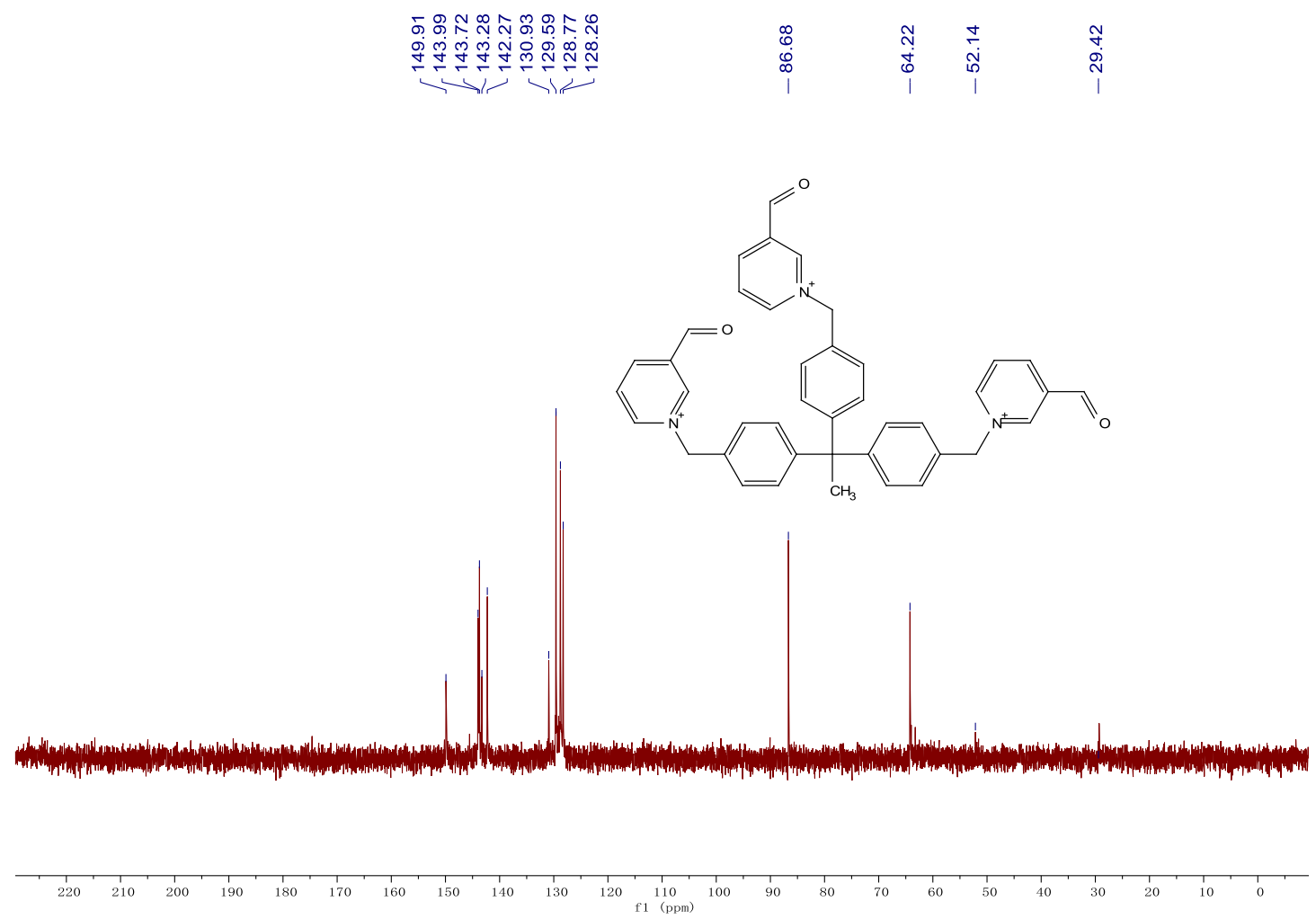

Figure S24. ${ }^{13} \mathrm{C}\left\{{ }^{1} \mathrm{H}\right\}$ NMR spectrum $(100 \mathrm{MHz})$ of $\mathbf{M 1}$ in $\mathrm{D}_{2} \mathrm{O}$ at $25{ }^{\circ} \mathrm{C}$. The signal of hydrated aldehyde corresponds to the peak with a chemical shift of $86.68 \mathrm{ppm}$.
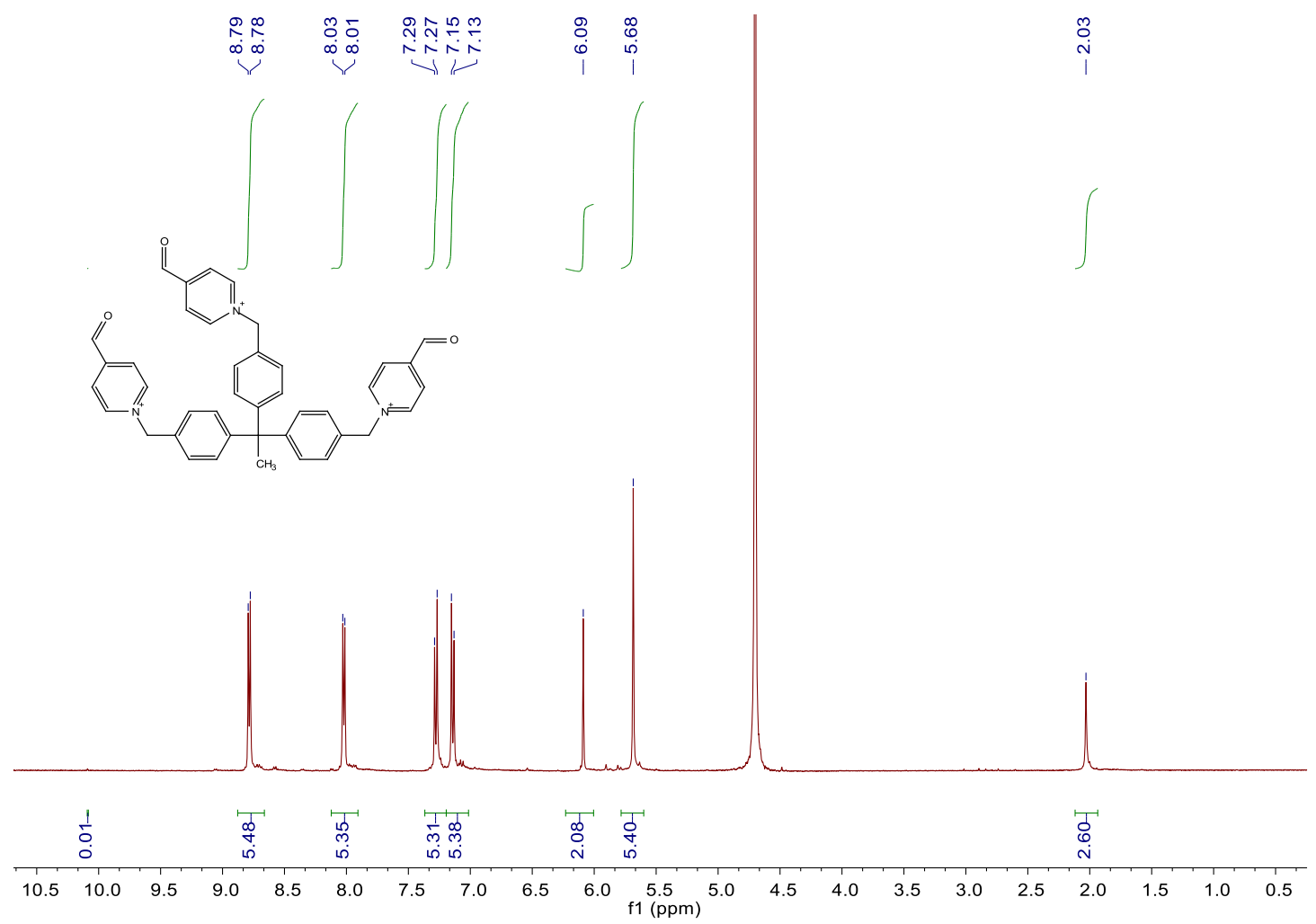

Figure S25. ${ }^{1} \mathrm{H}$ NMR spectrum $(400 \mathrm{MHz})$ of $\mathbf{M 2}$ in $\mathrm{D}_{2} \mathrm{O}$ at $25^{\circ} \mathrm{C} .99 \%$ of the aldehyde groups have been fully hydrated. 

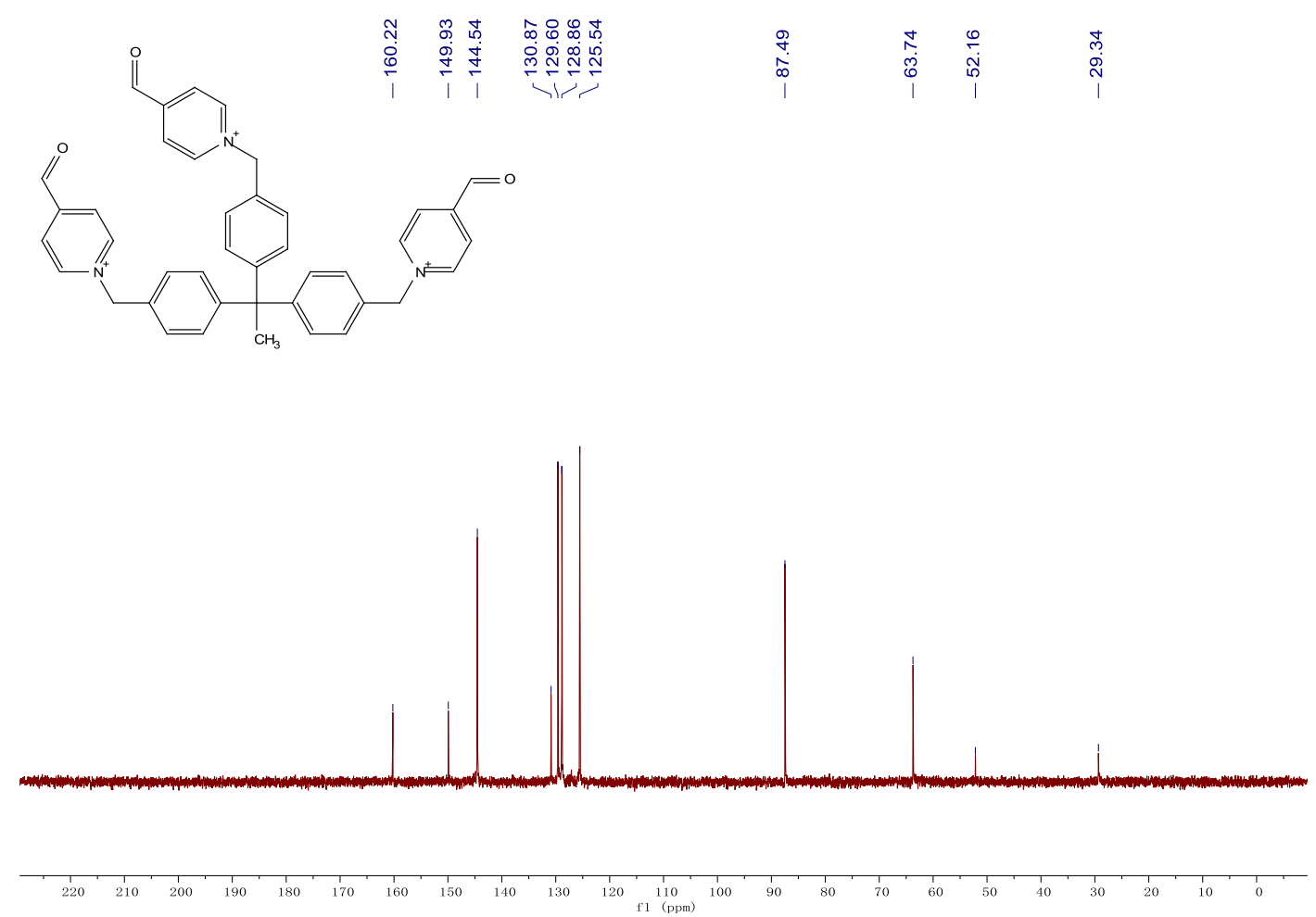

Figure S26. ${ }^{13} \mathrm{C}\left\{{ }^{1} \mathrm{H}\right\}$ NMR spectrum $(100 \mathrm{MHz})$ of $\mathbf{M} 2$ in $\mathrm{D}_{2} \mathrm{O}$ at $25{ }^{\circ} \mathrm{C}$. The signal of hydrated aldehyde corresponds to the peak with a chemical shift of $87.49 \mathrm{ppm}$.

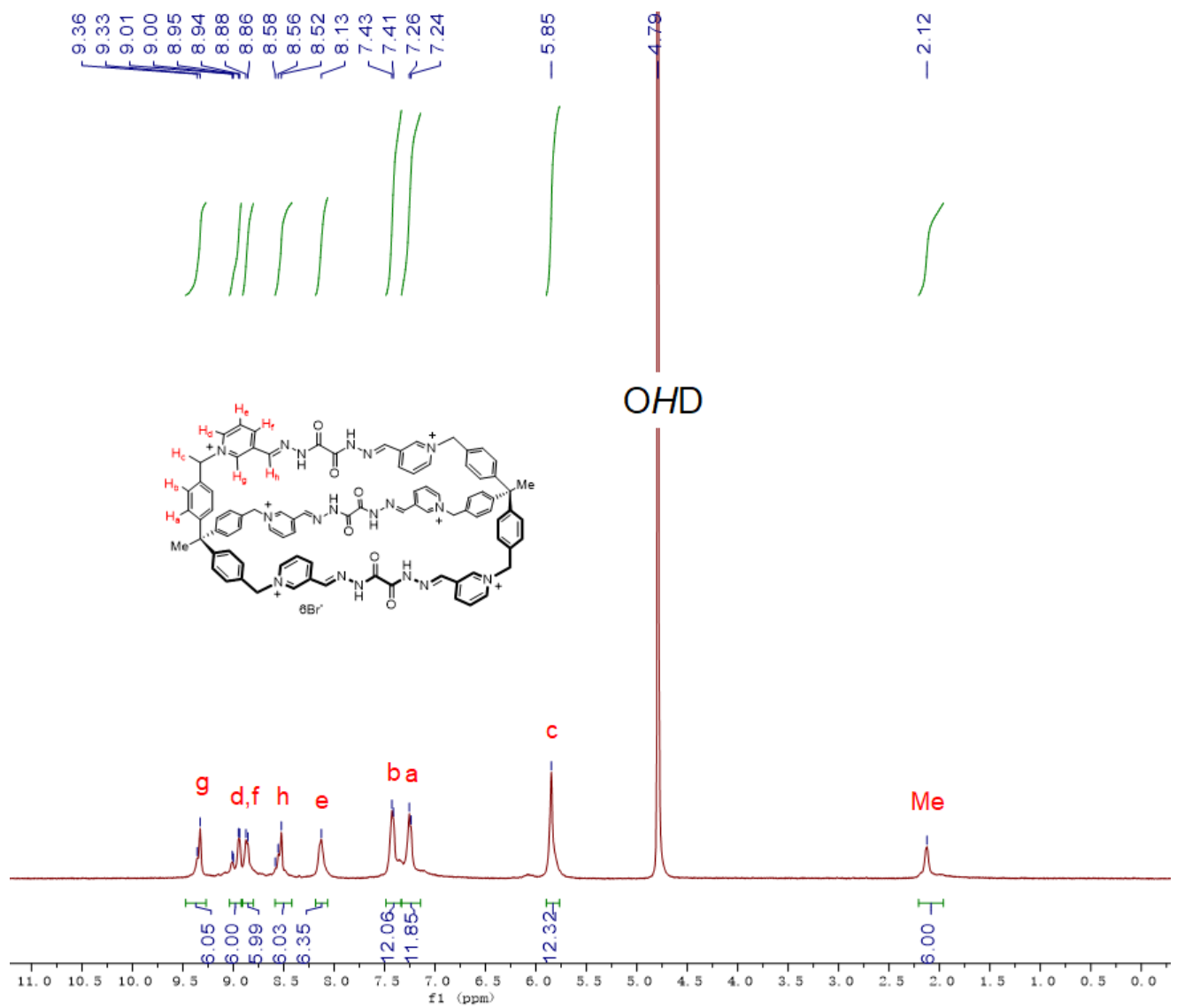

Figure S27. ${ }^{1} \mathrm{H}$ NMR spectrum $(400 \mathrm{MHz})$ of $\mathbf{C 1}$ in $\mathrm{D}_{2} \mathrm{O}$ at $25^{\circ} \mathrm{C}$. 


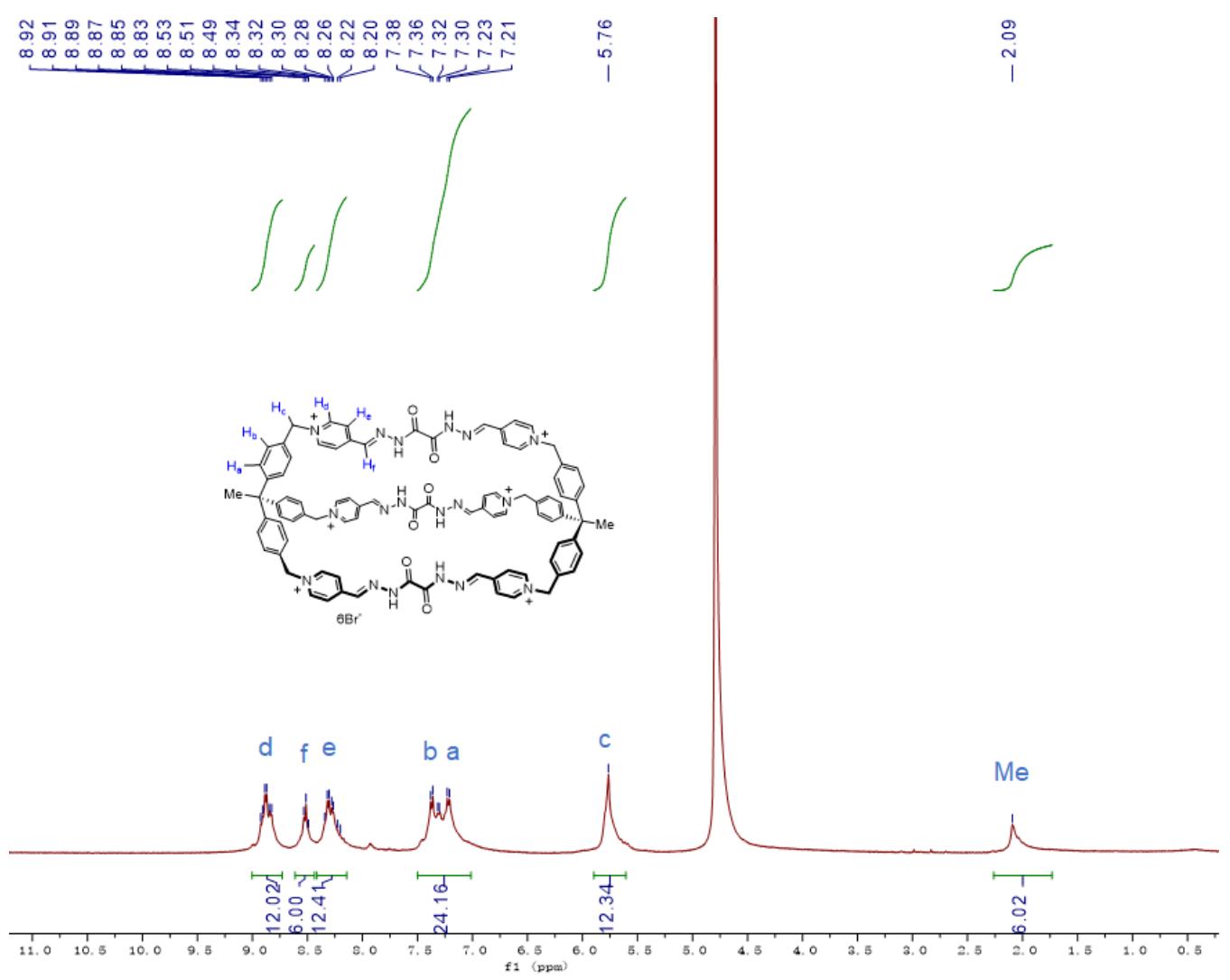

Figure S28. ${ }^{1} \mathrm{H}$ NMR spectrum $(400 \mathrm{MHz})$ of $\mathbf{C 2}$ in $\mathrm{D}_{2} \mathrm{O}$ at $25{ }^{\circ} \mathrm{C}$.
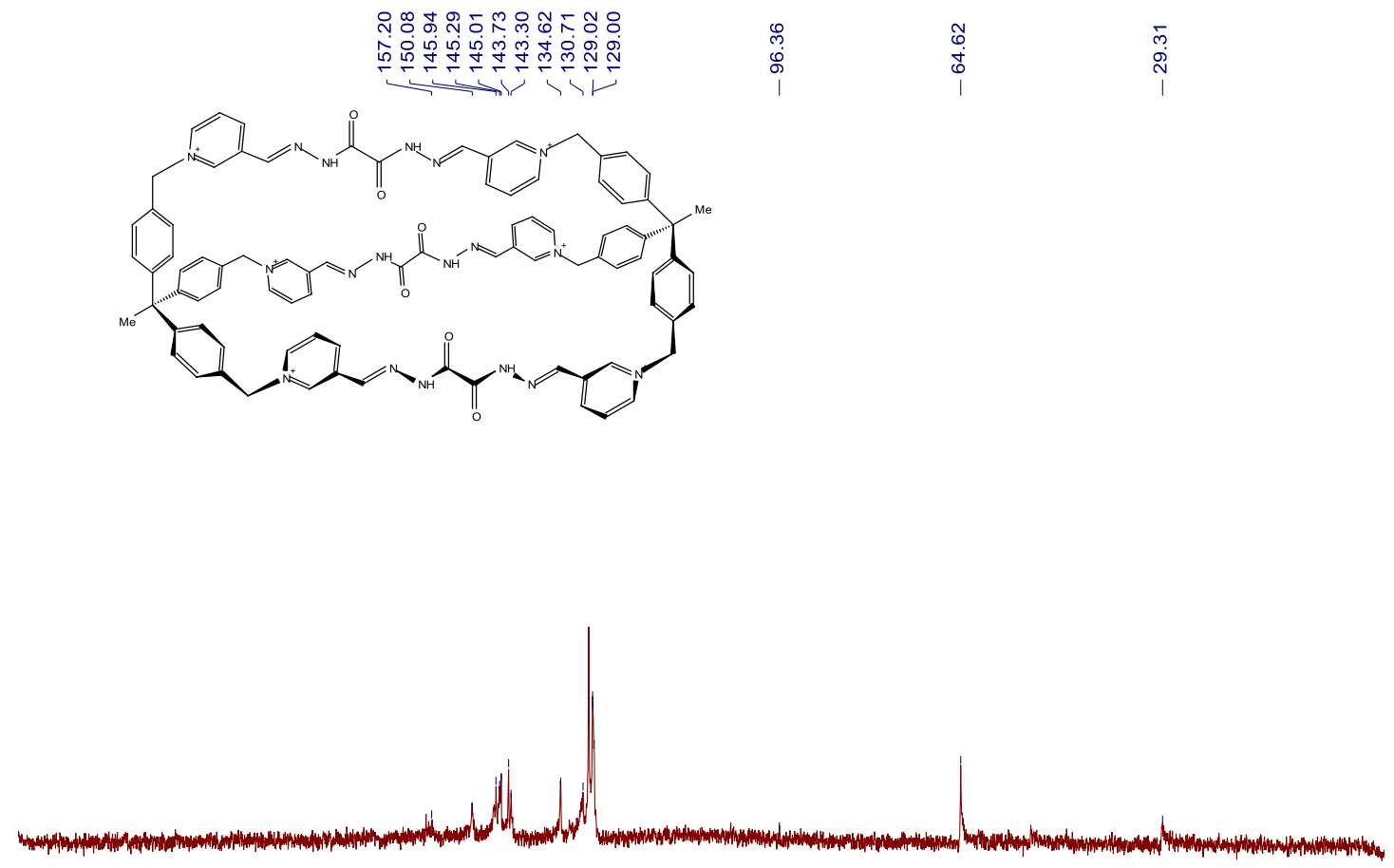

Figure S29. ${ }^{13} \mathrm{C}\left\{{ }^{1} \mathrm{H}\right\}$ NMR spectrum $(100 \mathrm{MHz})$ of $\mathbf{C 1}$ in $\mathrm{D}_{2} \mathrm{O}$ at $25{ }^{\circ} \mathrm{C}$. 

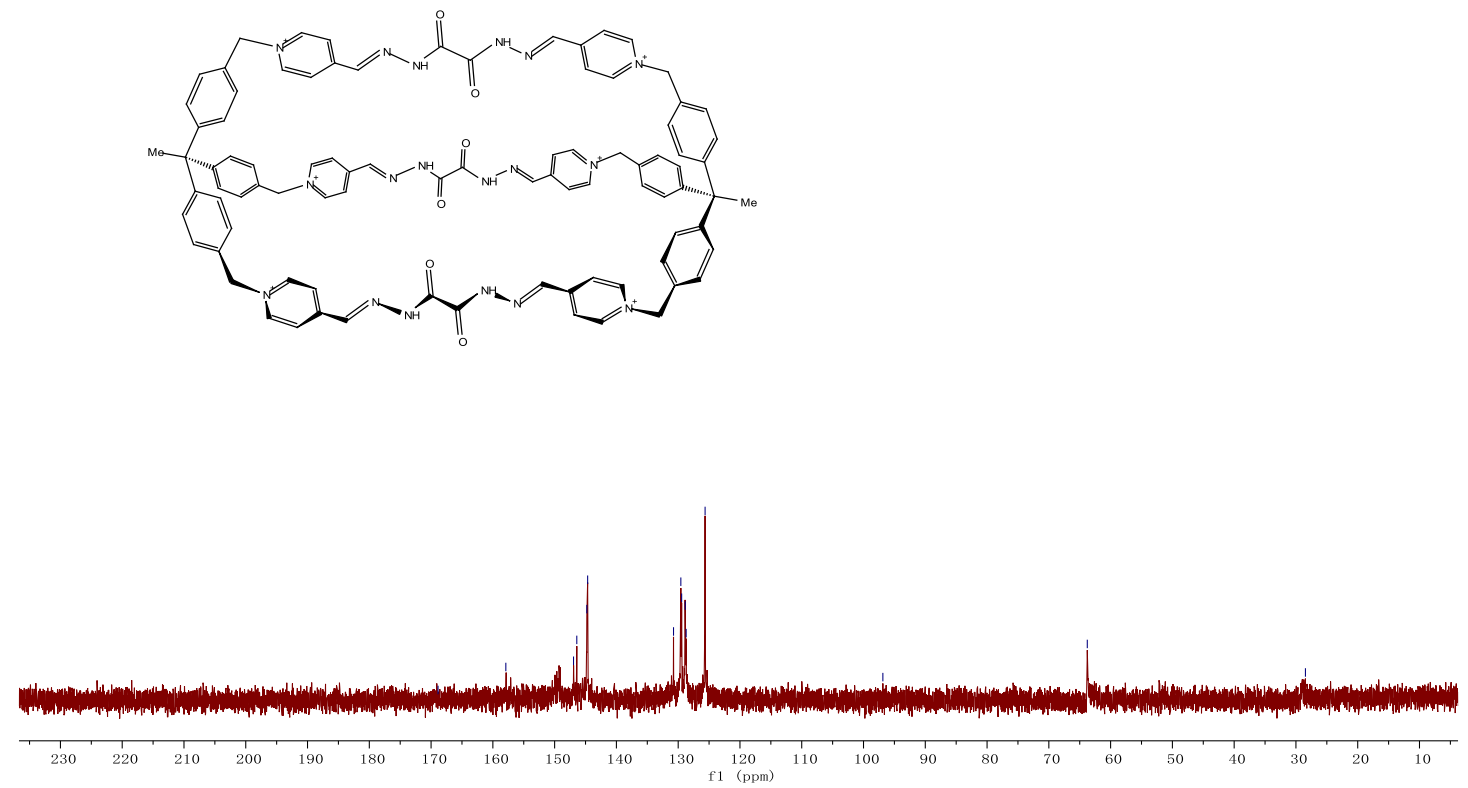

Figure S30. ${ }^{13} \mathrm{C}\left\{{ }^{1} \mathrm{H}\right\}$ NMR spectrum $(100 \mathrm{MHz})$ of $\mathbf{C} 2$ in $\mathrm{D}_{2} \mathrm{O}$ at $25{ }^{\circ} \mathrm{C}$.

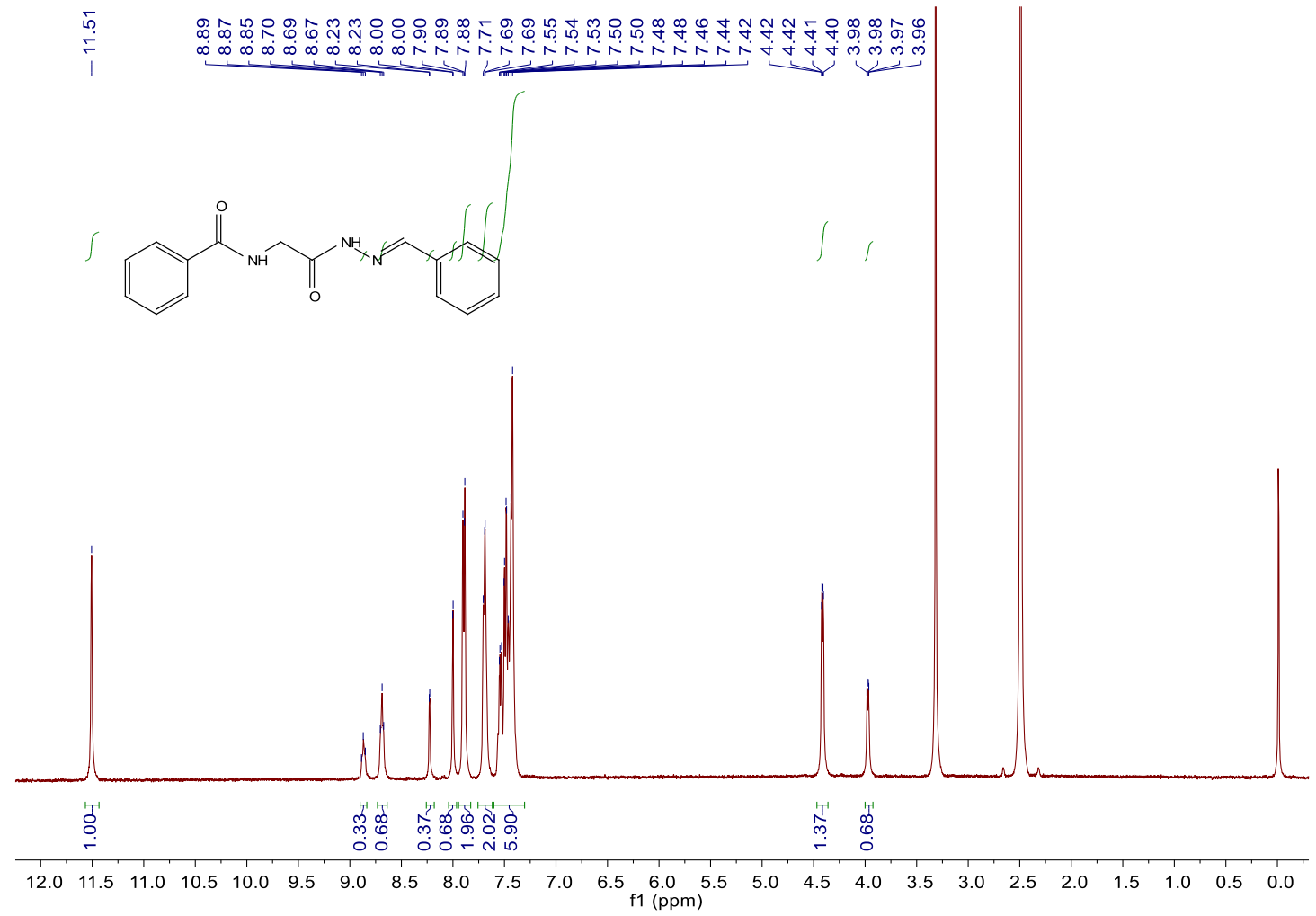

Figure S31. ${ }^{1} \mathrm{H}$ NMR spectrum $\left(400 \mathrm{MHz}, \mathrm{DMSO}_{-} \mathrm{d}_{6}, 25^{\circ} \mathrm{C}\right)$ of $7 \mathbf{a}$. 


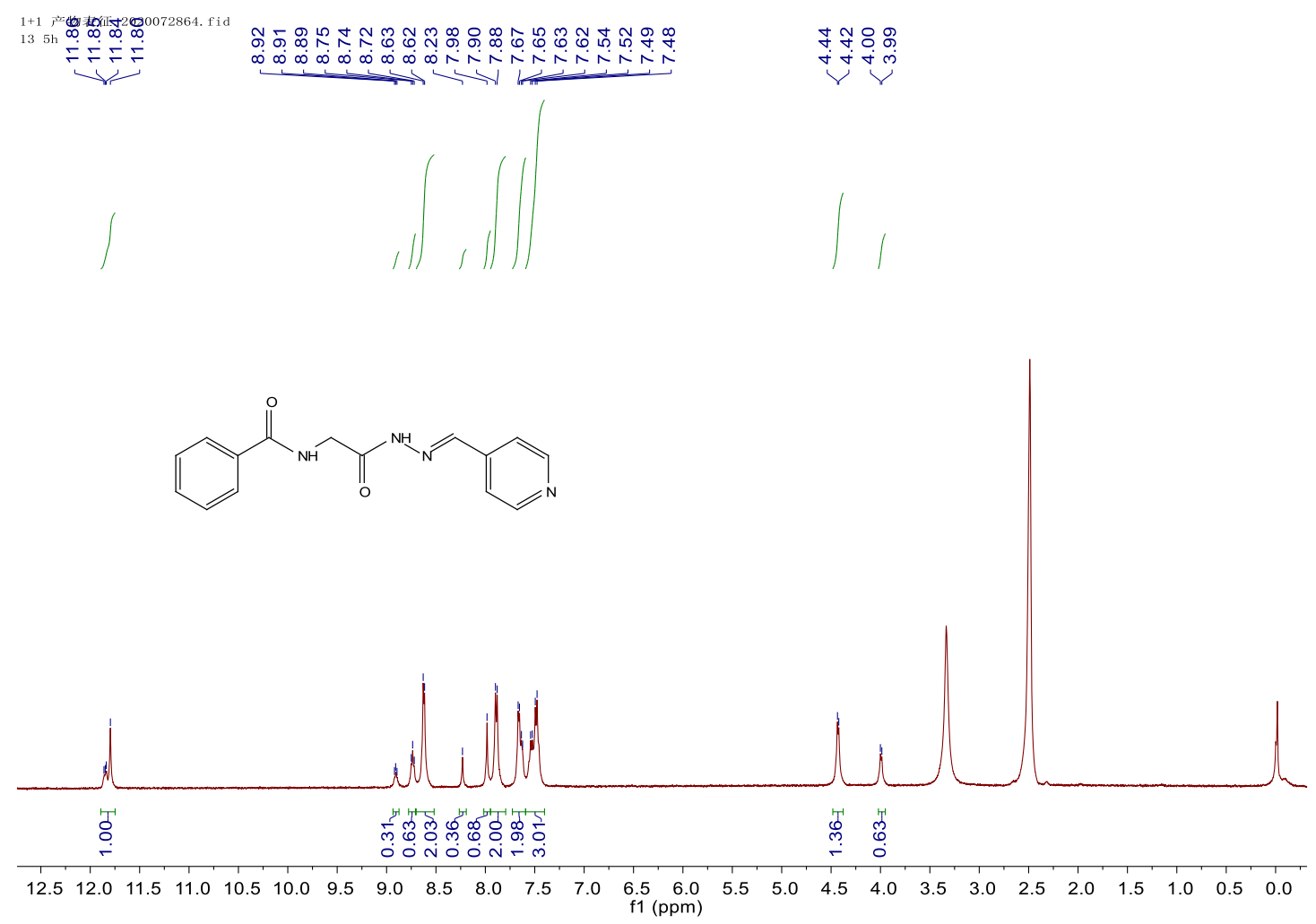

Figure S32. ${ }^{1} \mathrm{H}$ NMR spectrum (400 MHz, DMSO-d $\left.6,25^{\circ} \mathrm{C}\right)$ of $7 \mathbf{b}$.

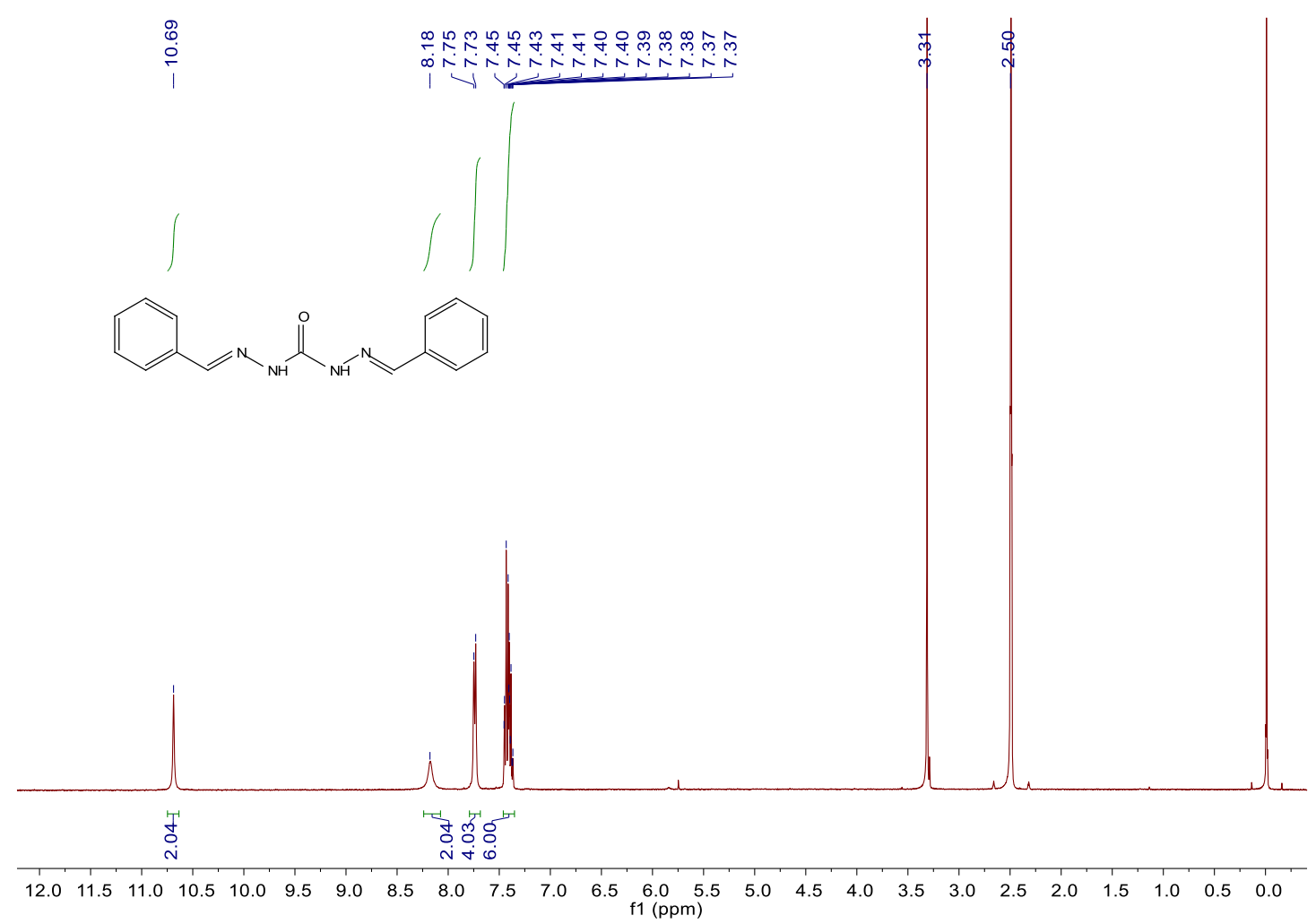

Figure S33. ${ }^{1} \mathrm{H}$ NMR spectrum (400 MHz, DMSO-d $6,25{ }^{\circ} \mathrm{C}$ ) of 9a. 


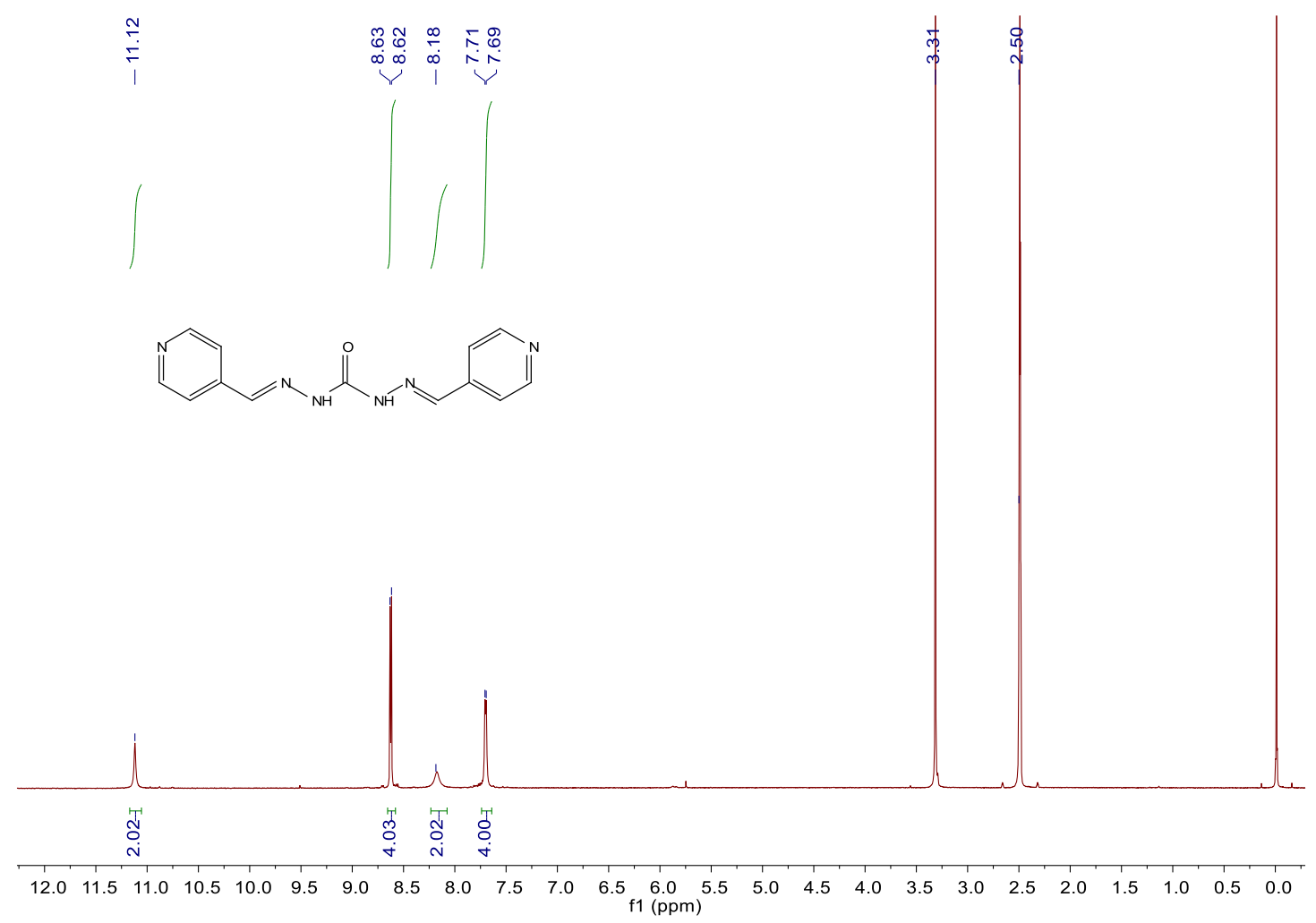

Figure S34. ${ }^{1} \mathrm{H}$ NMR spectrum (400 MHz, DMSO-d $\left.6,25^{\circ} \mathrm{C}\right)$ of $9 \mathbf{b}$.

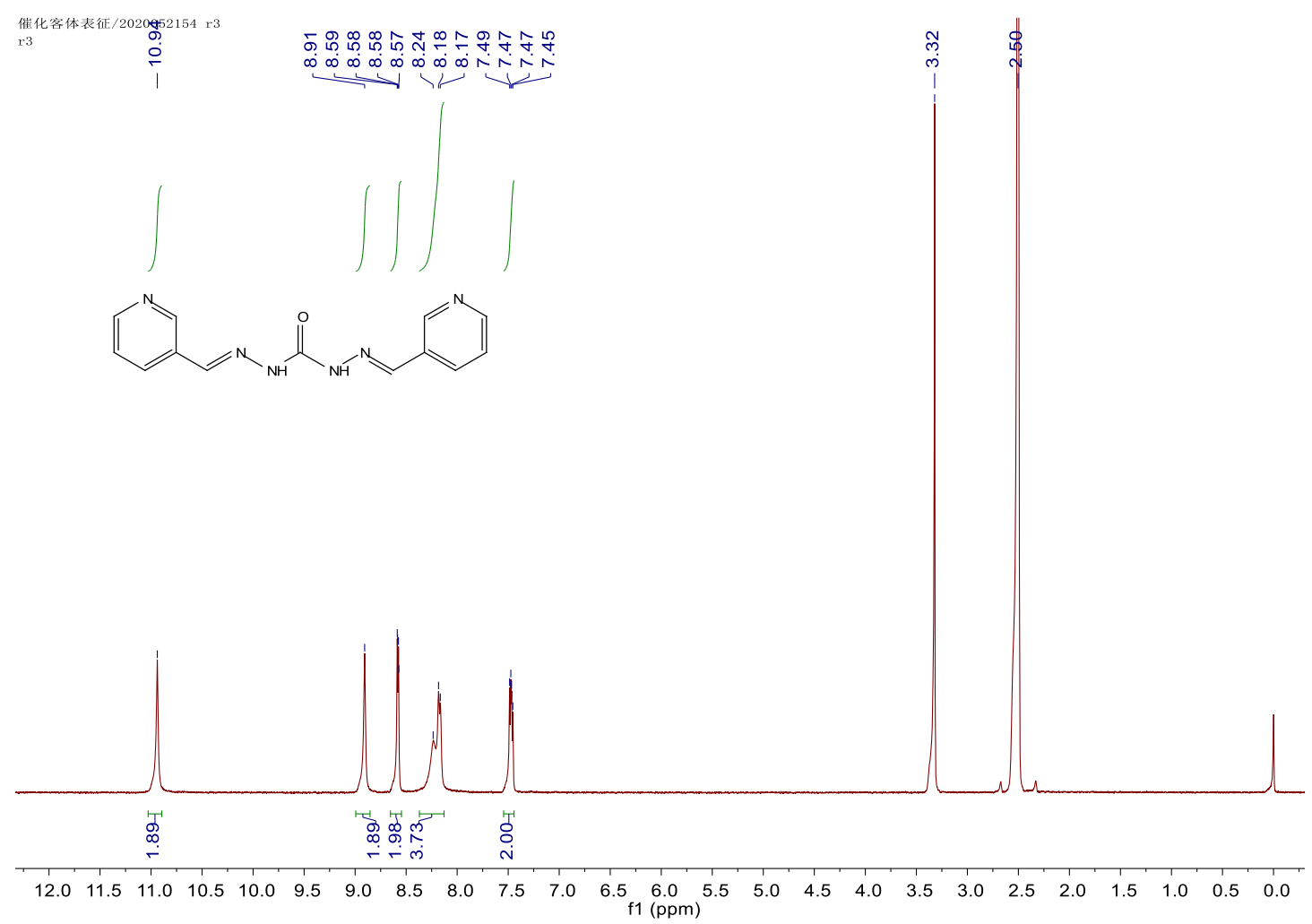

Figure S35. ${ }^{1} \mathrm{H}$ NMR spectrum $\left(400 \mathrm{MHz}, \mathrm{DMSO}-\mathrm{d}_{6}, 25^{\circ} \mathrm{C}\right)$ of $9 \mathrm{c}$. 


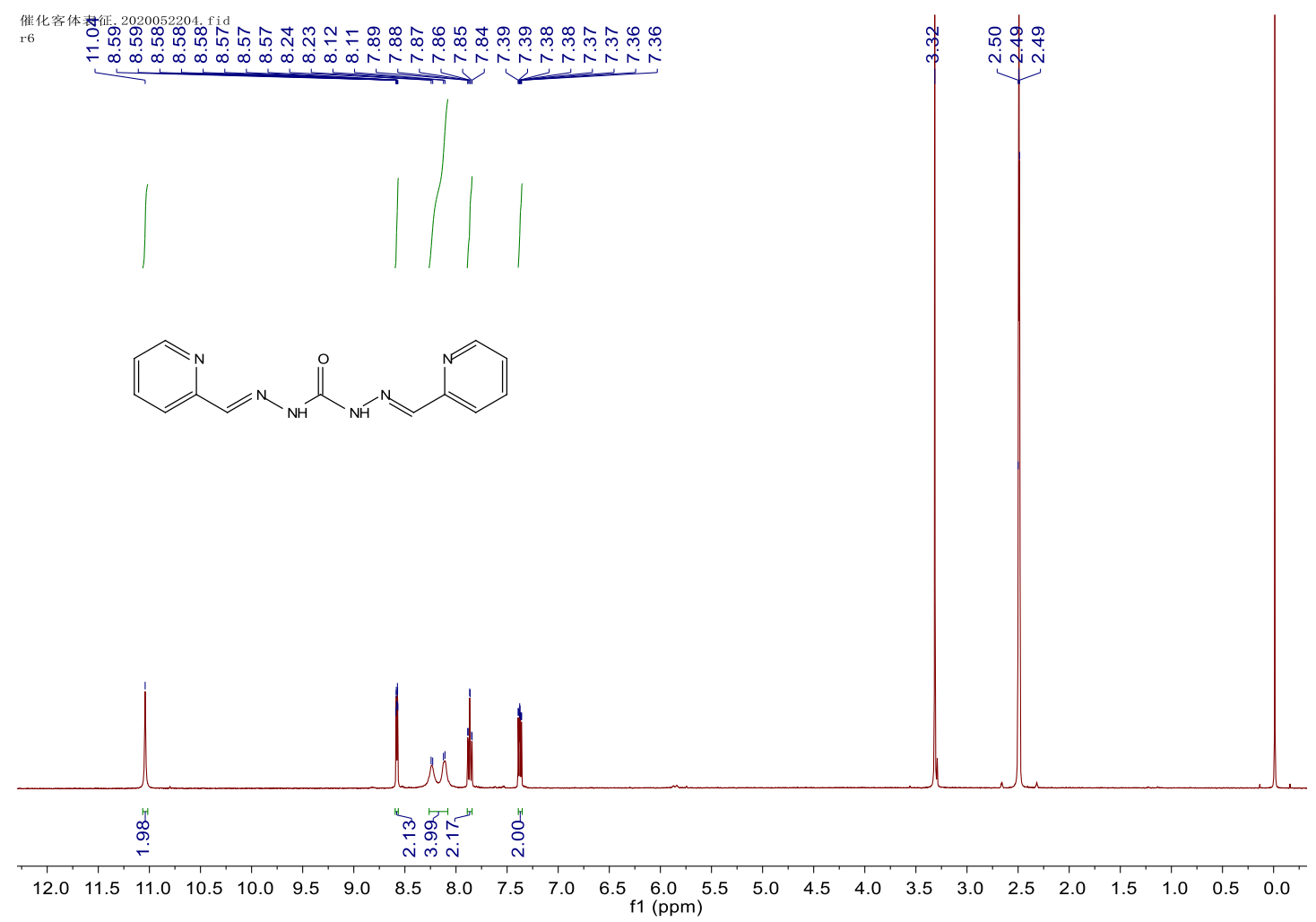

Figure S36. ${ }^{1} \mathrm{H}$ NMR spectrum $\left(400 \mathrm{MHz}, \mathrm{DMSO}-\mathrm{d}_{6}, 25^{\circ} \mathrm{C}\right)$ of $9 d$.

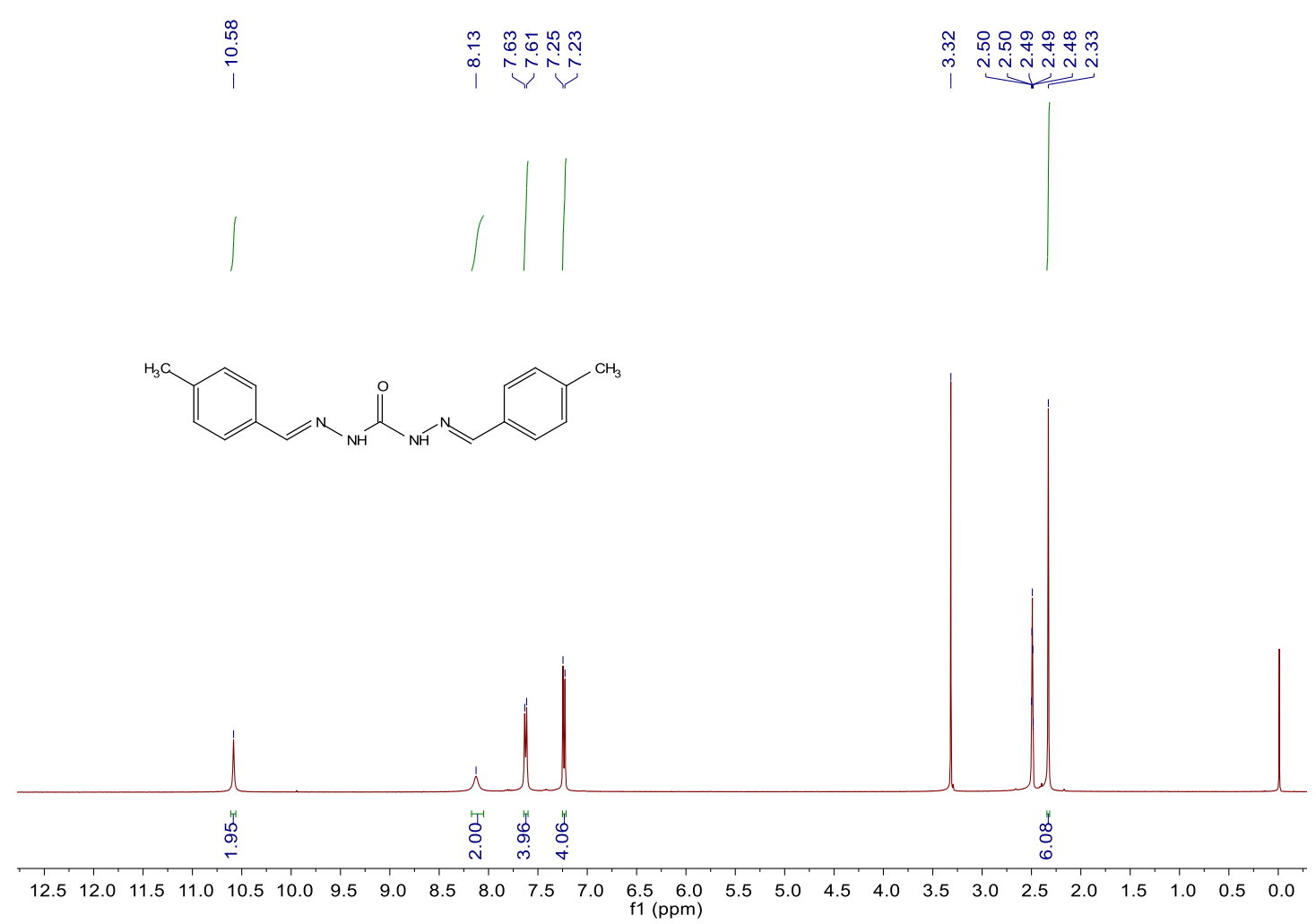

Figure S37. ${ }^{1} \mathrm{H}$ NMR spectrum $\left(400 \mathrm{MHz}\right.$, DMSO-d $\left.6,25^{\circ} \mathrm{C}\right)$ of $9 e$. 


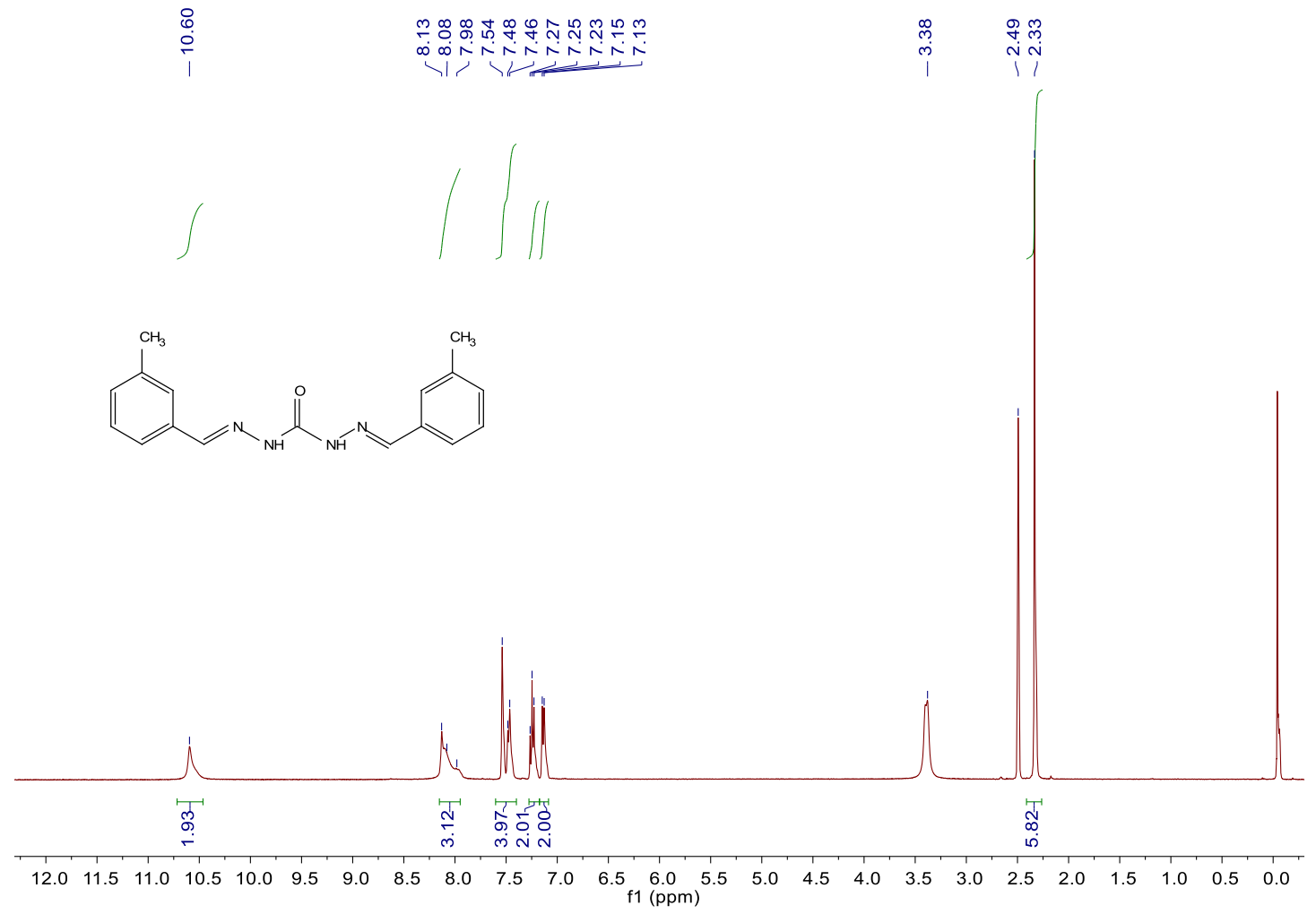

Figure S38. ${ }^{1} \mathrm{H}$ NMR spectrum (400 MHz, DMSO-d $\left.6,25^{\circ} \mathrm{C}\right)$ of $9 f$.

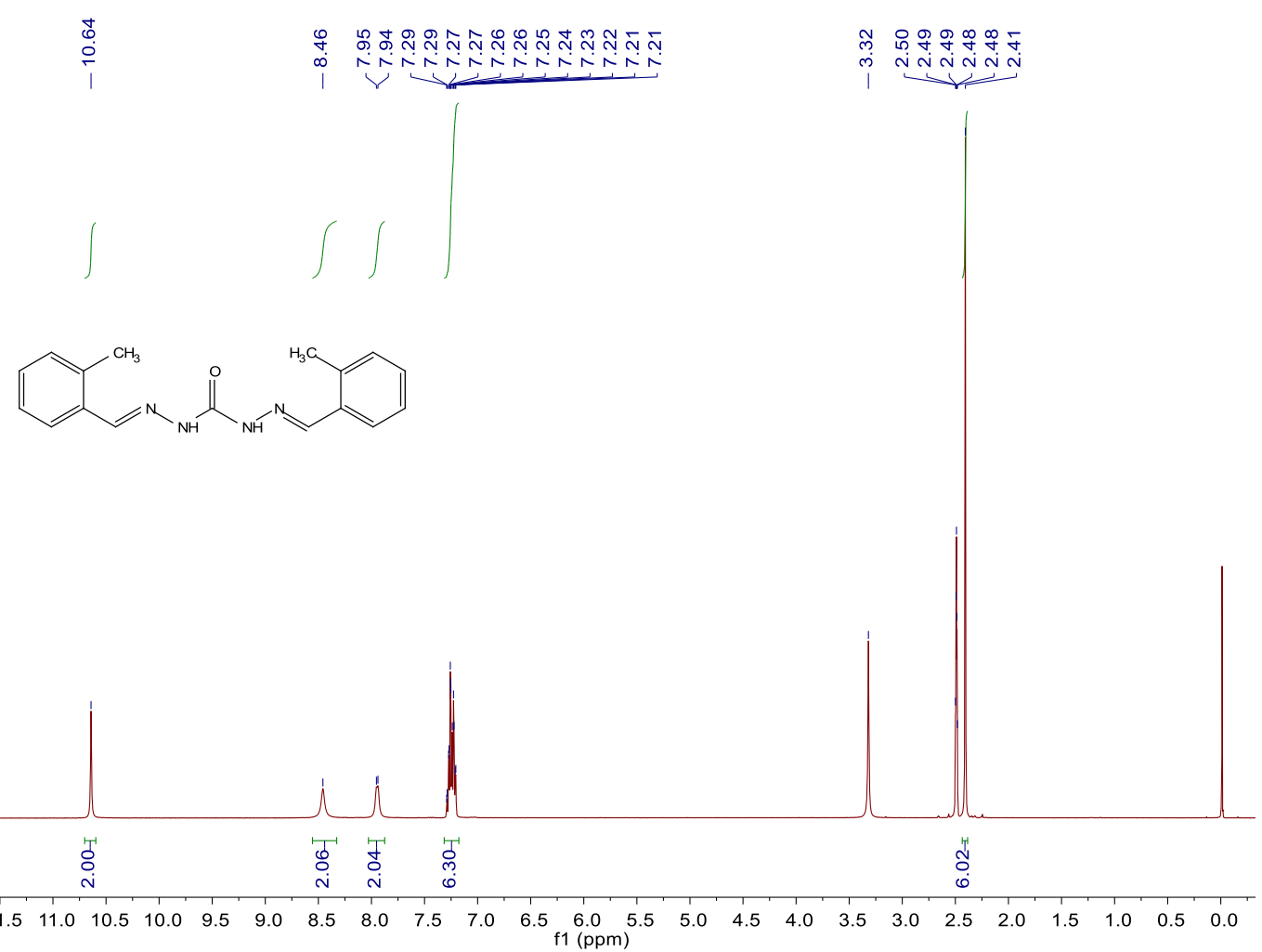

Figure S39. ${ }^{1} \mathrm{H}$ NMR spectrum $\left(400 \mathrm{MHz}, \mathrm{DMSO}-\mathrm{d}_{6}, 25^{\circ} \mathrm{C}\right)$ of $9 \mathrm{~g}$. 


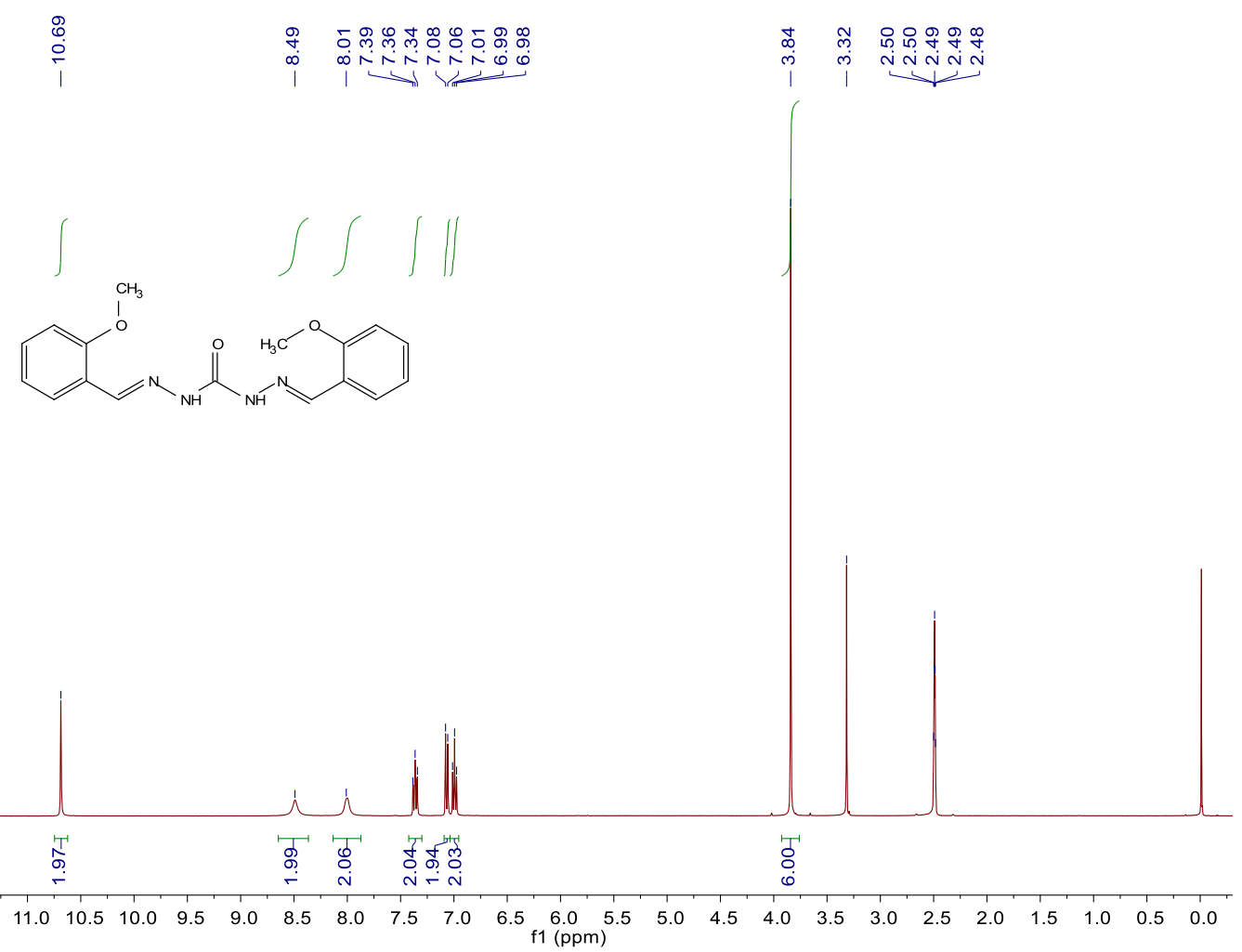

Figure S40. ${ }^{1} \mathrm{H}$ NMR spectrum $\left(400 \mathrm{MHz}, \mathrm{DMSO}_{-} \mathrm{d}_{6}, 25^{\circ} \mathrm{C}\right)$ of $\mathbf{9 h}$.

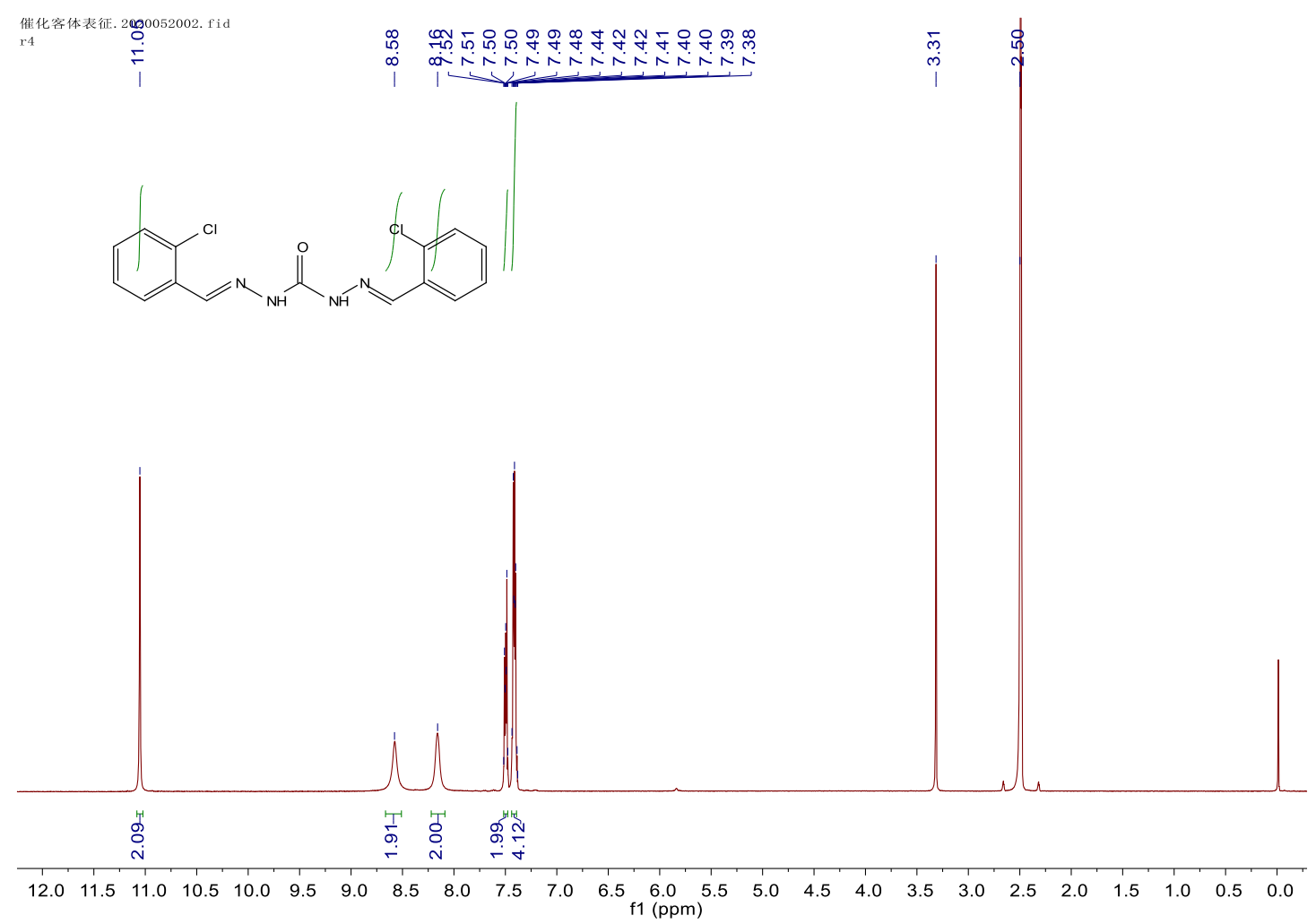

Figure S41. ${ }^{1} \mathrm{H}$ NMR spectrum $\left(400 \mathrm{MHz}, \mathrm{DMSO}-\mathrm{d}_{6}, 25^{\circ} \mathrm{C}\right)$ of $9 \mathrm{i}$. 


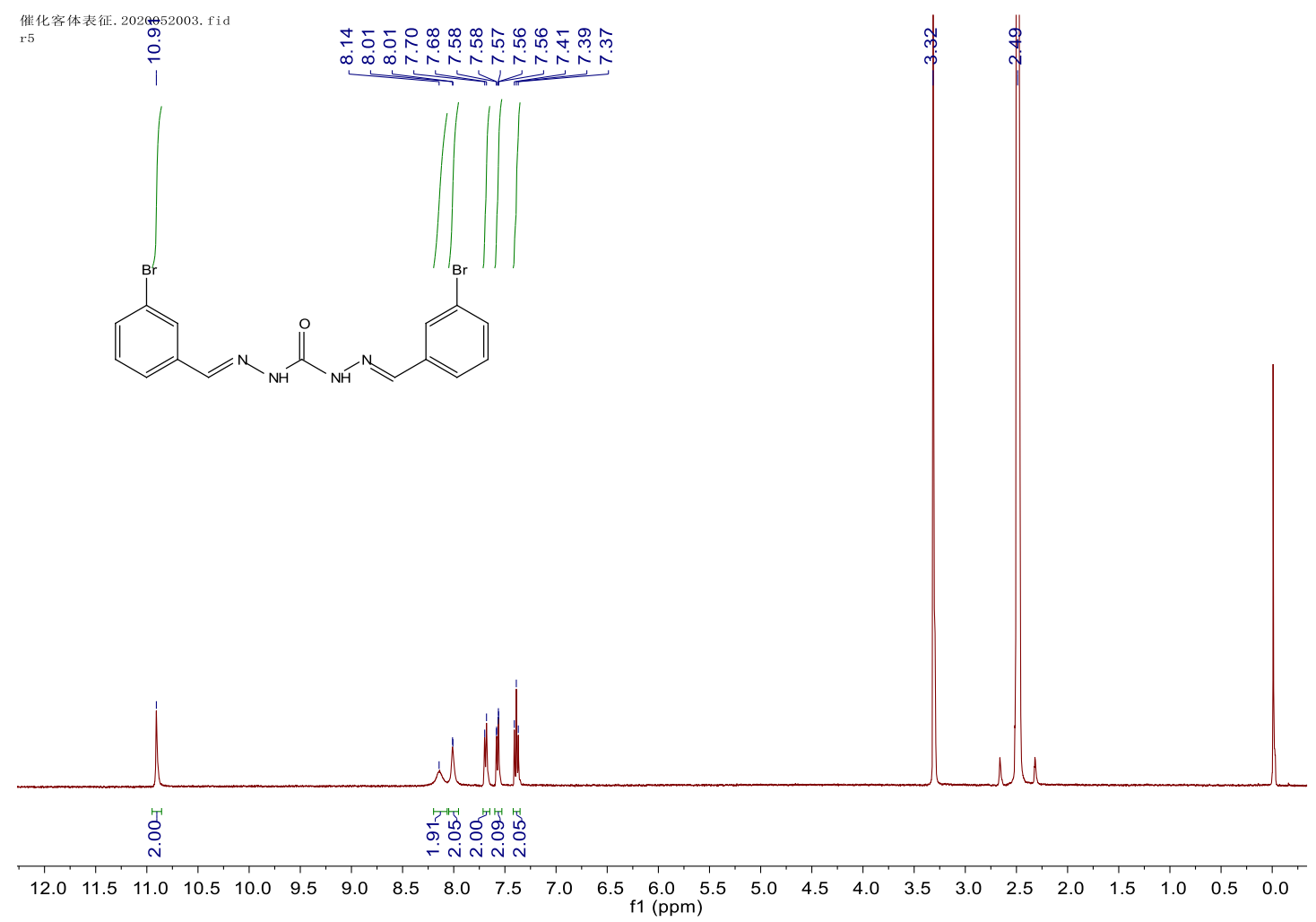

Figure S42. ${ }^{1} \mathrm{H}$ NMR spectrum (400 MHz, DMSO-d $\left.6,25^{\circ} \mathrm{C}\right)$ of $\mathbf{9 j}$.

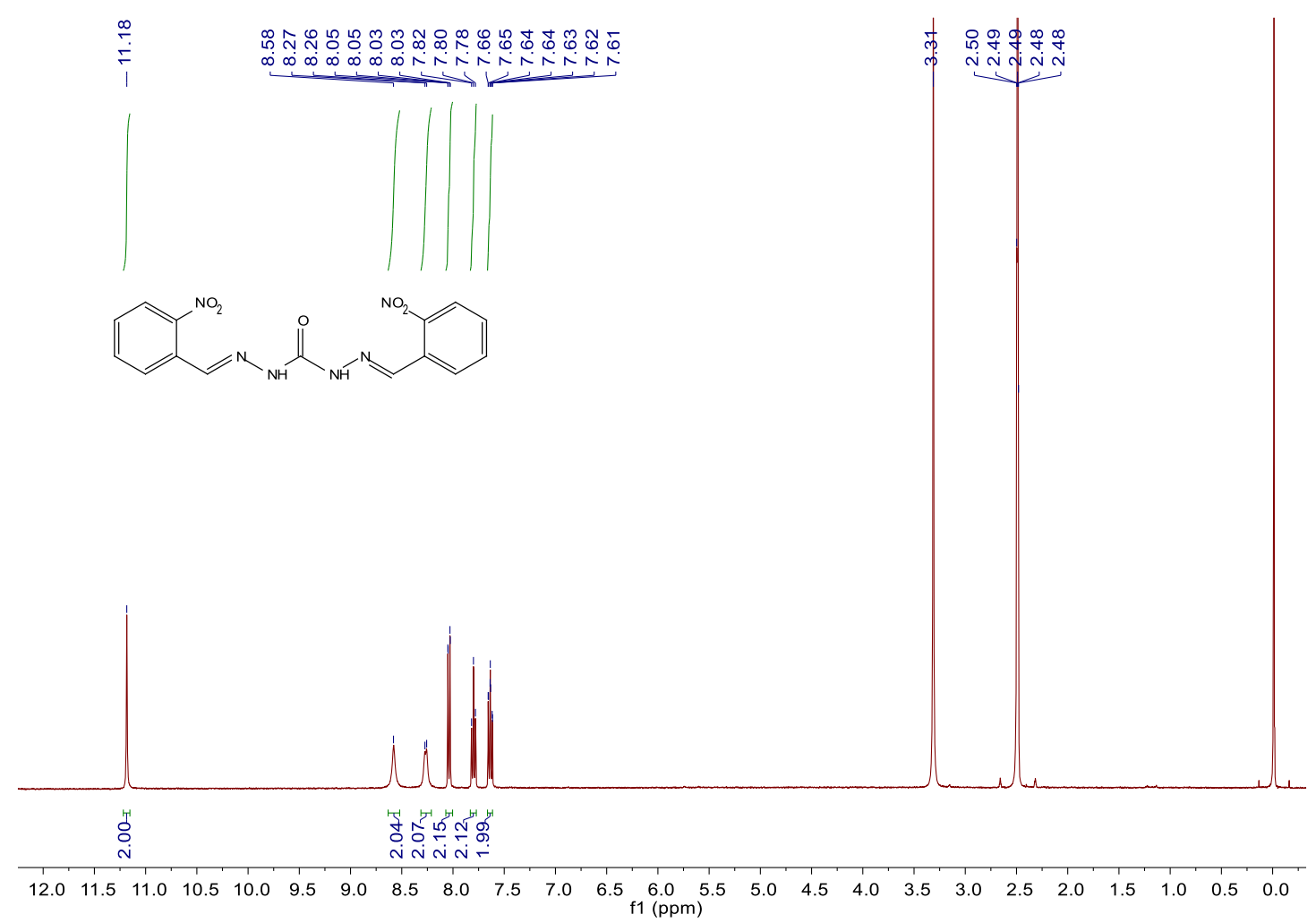

Figure S43. ${ }^{1} \mathrm{H}$ NMR spectrum (400 MHz, DMSO-d $\left.6,25{ }^{\circ} \mathrm{C}\right)$ of $9 k$. 


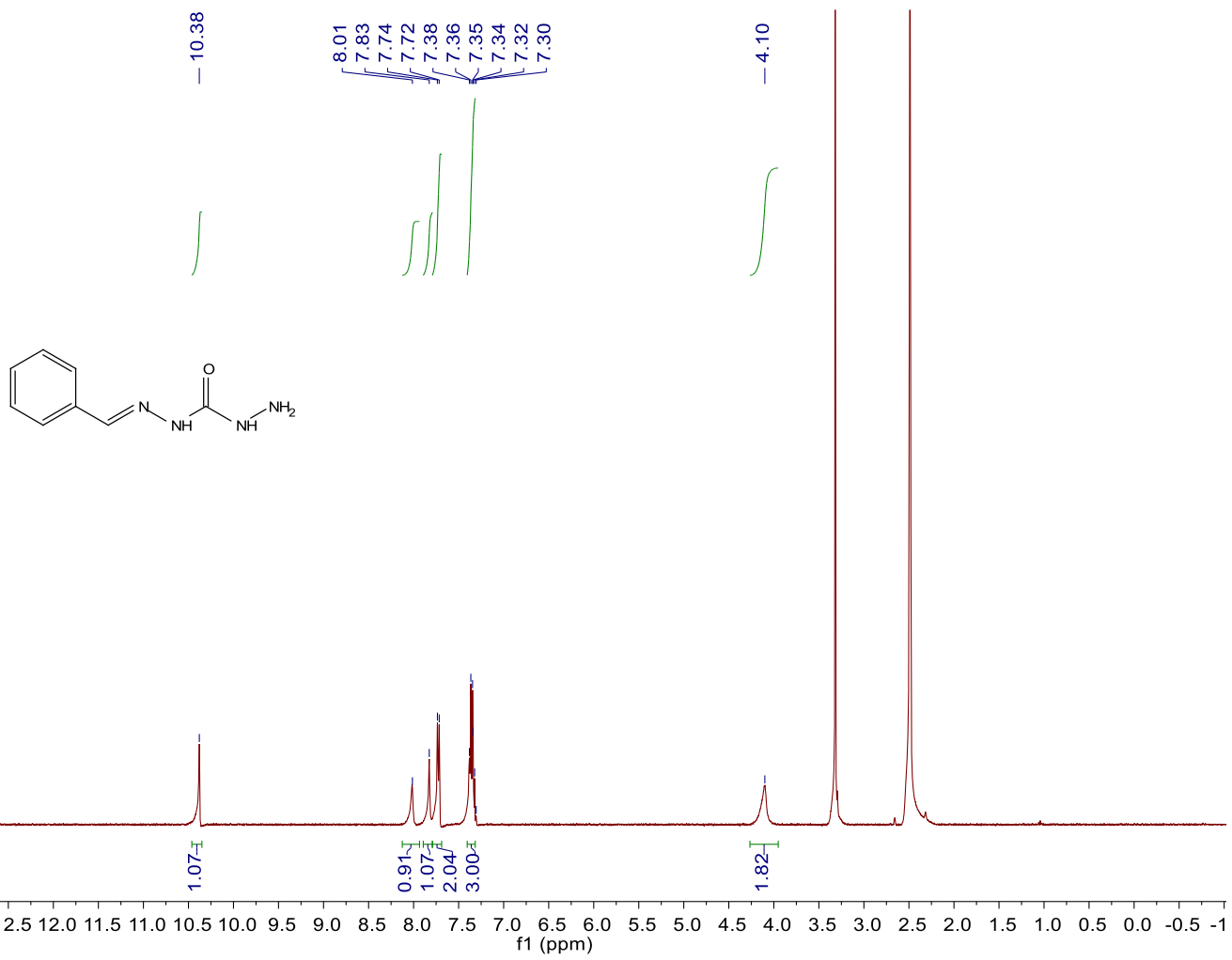

Figure S44. ${ }^{1} \mathrm{H}$ NMR spectrum (400 MHz, DMSO-d $6,25^{\circ} \mathrm{C}$ ) of 10a.

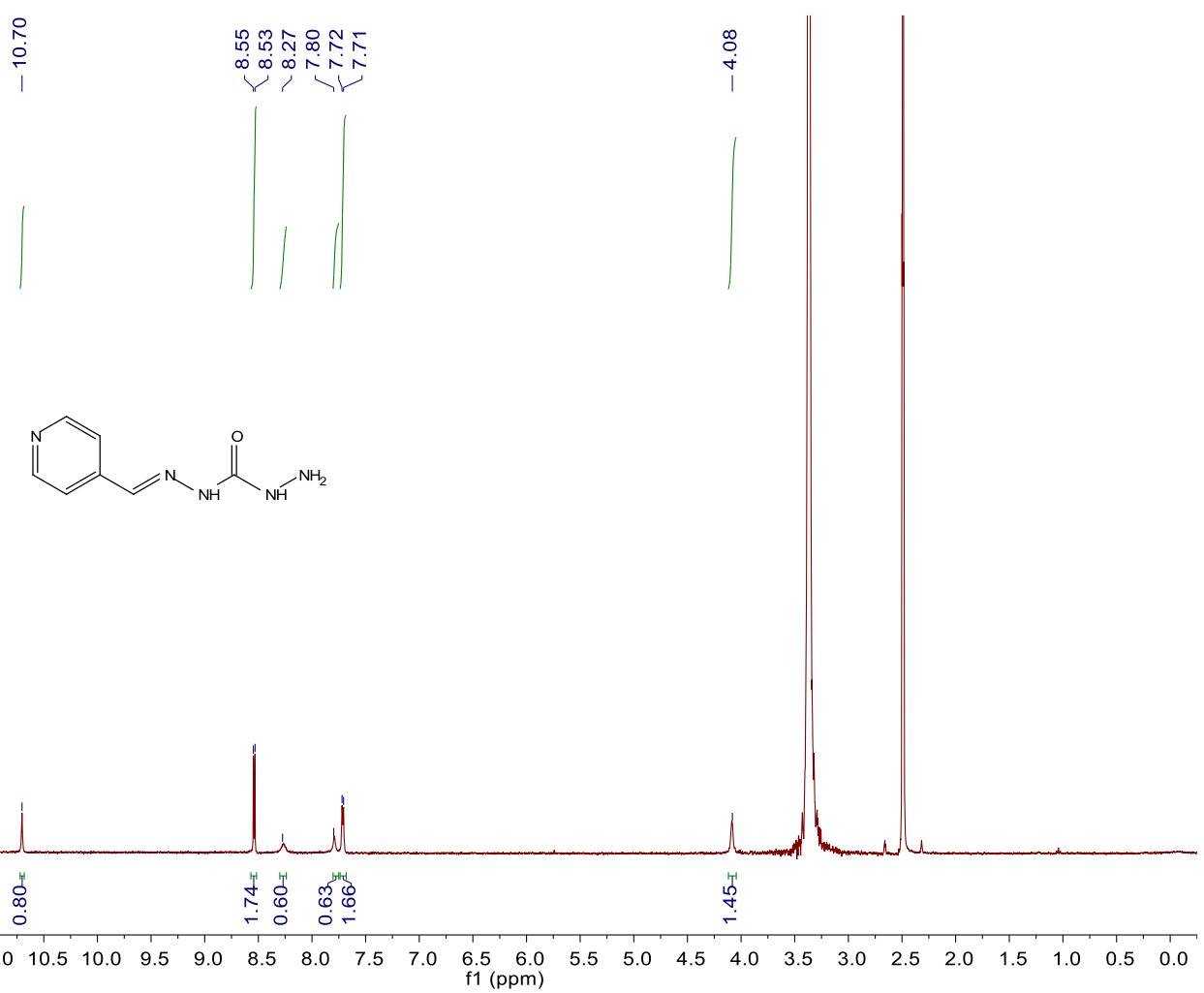

Figure S45. ${ }^{1} \mathrm{H}$ NMR spectrum (400 MHz, DMSO-d $6,25{ }^{\circ} \mathrm{C}$ ) of $\mathbf{1 0 b}$. 


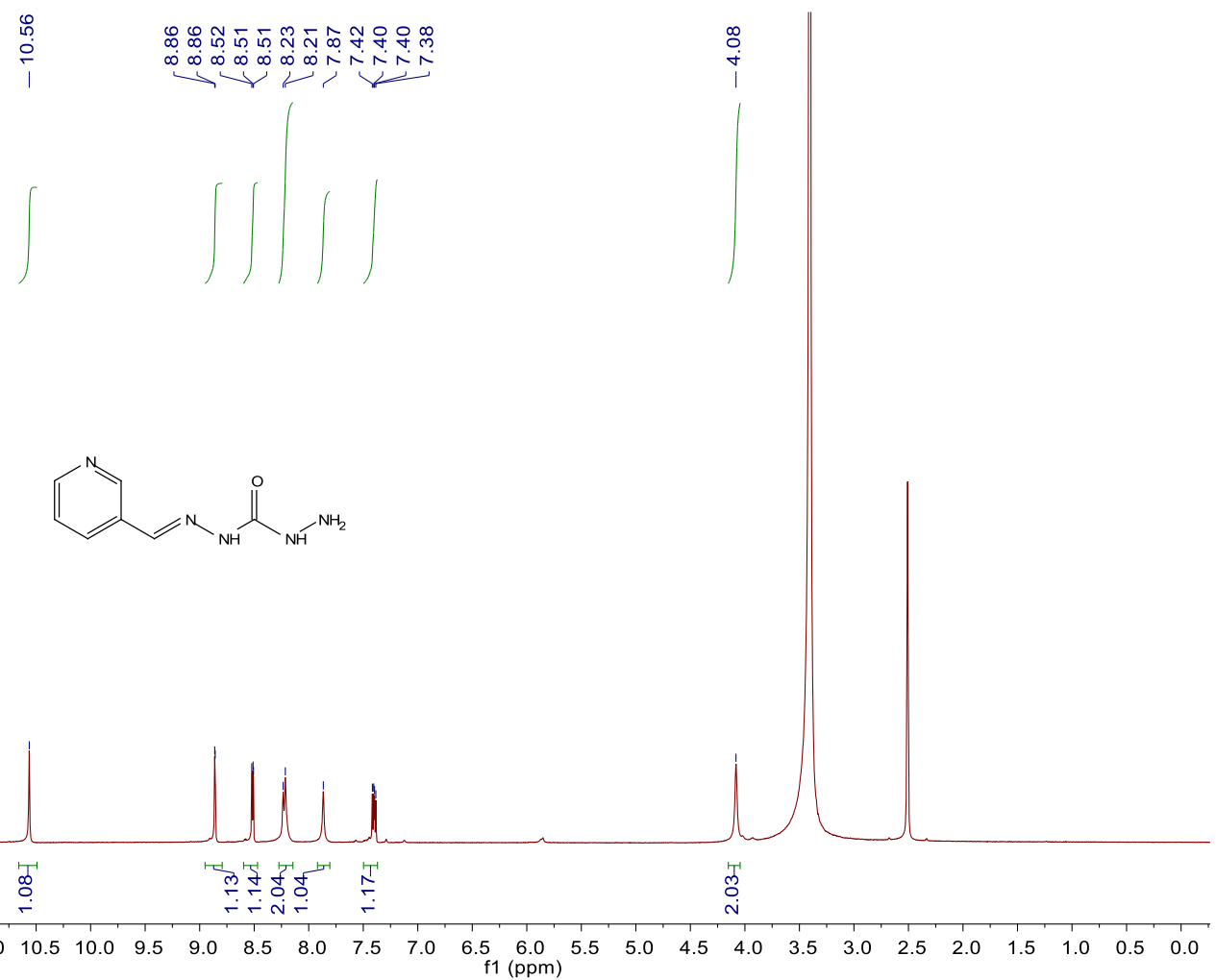

Figure S46. ${ }^{1} \mathrm{H}$ NMR spectrum (400 MHz, DMSO-d $6,25^{\circ} \mathrm{C}$ ) of 10c.

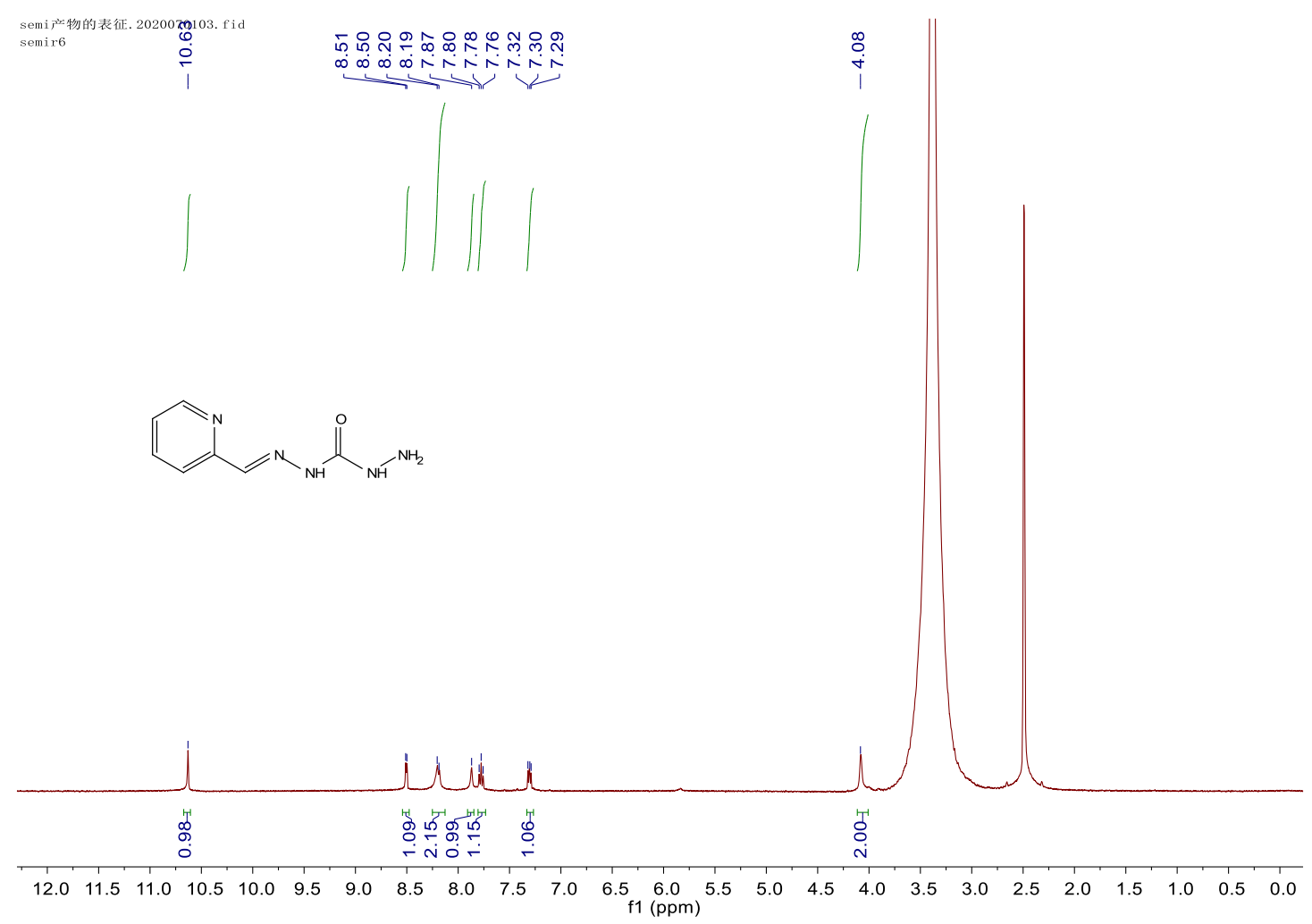

Figure S47. ${ }^{1} \mathrm{H}$ NMR spectrum (400 MHz, DMSO-d $6,25^{\circ} \mathrm{C}$ ) of $\mathbf{1 0 d}$. 


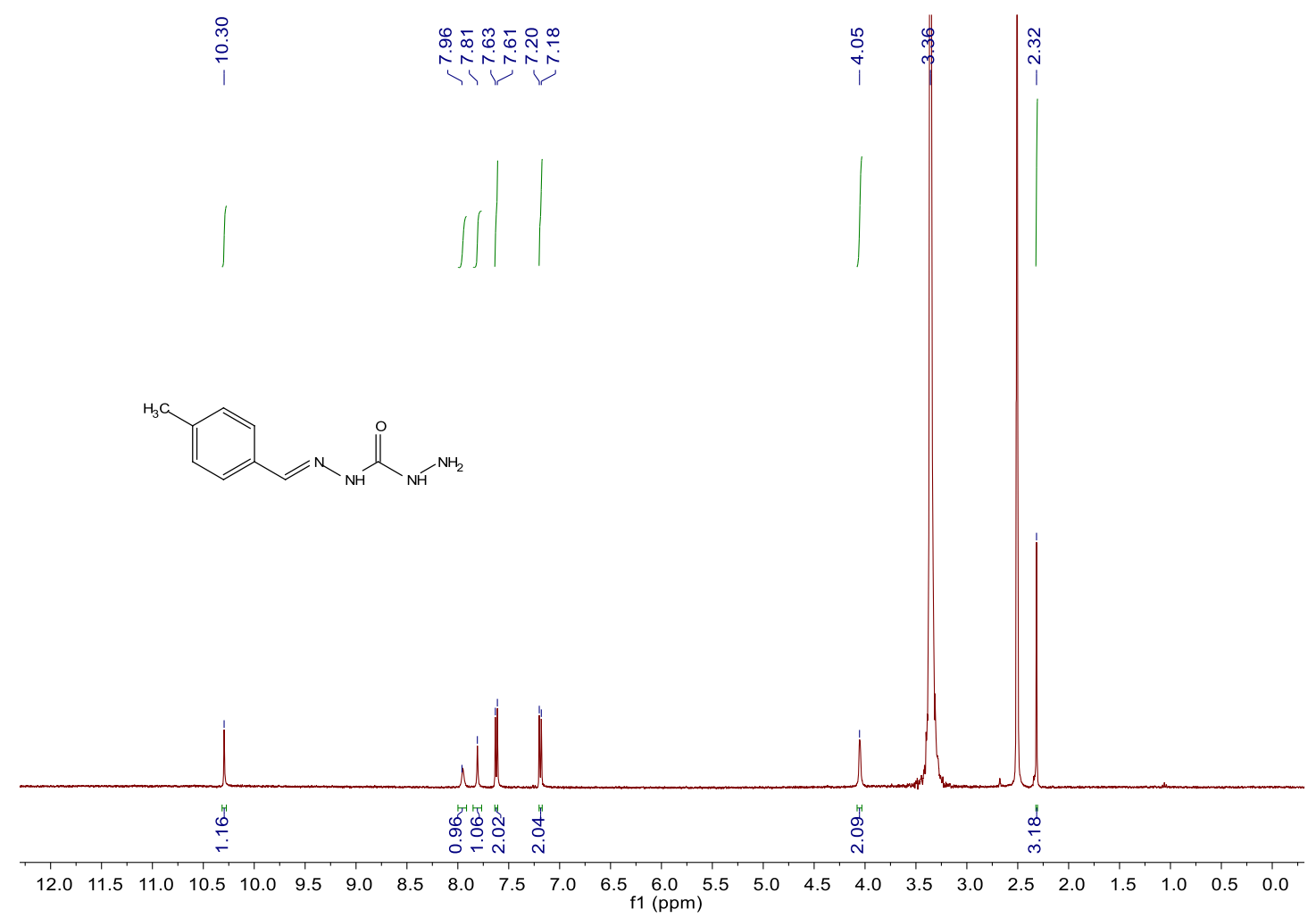

Figure S48. ${ }^{1} \mathrm{H}$ NMR spectrum (400 MHz, DMSO-d $6,25^{\circ} \mathrm{C}$ ) of $\mathbf{1 0 e}$.

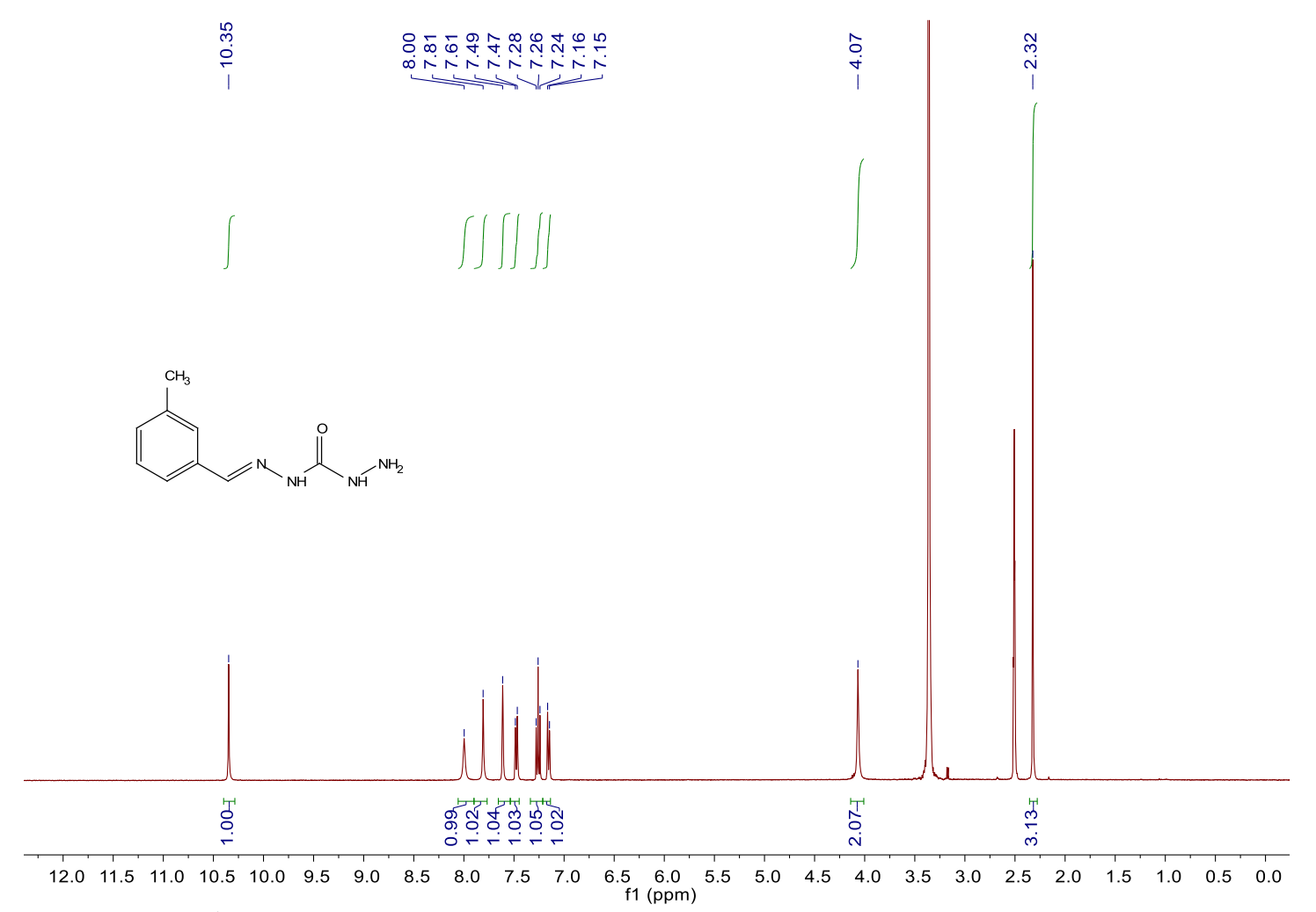

Figure S49. ${ }^{1} \mathrm{H}$ NMR spectrum $\left(400 \mathrm{MHz}, \mathrm{DMSO}-\mathrm{d}_{6}, 25{ }^{\circ} \mathrm{C}\right.$ ) of $\mathbf{1 0 f}$. 


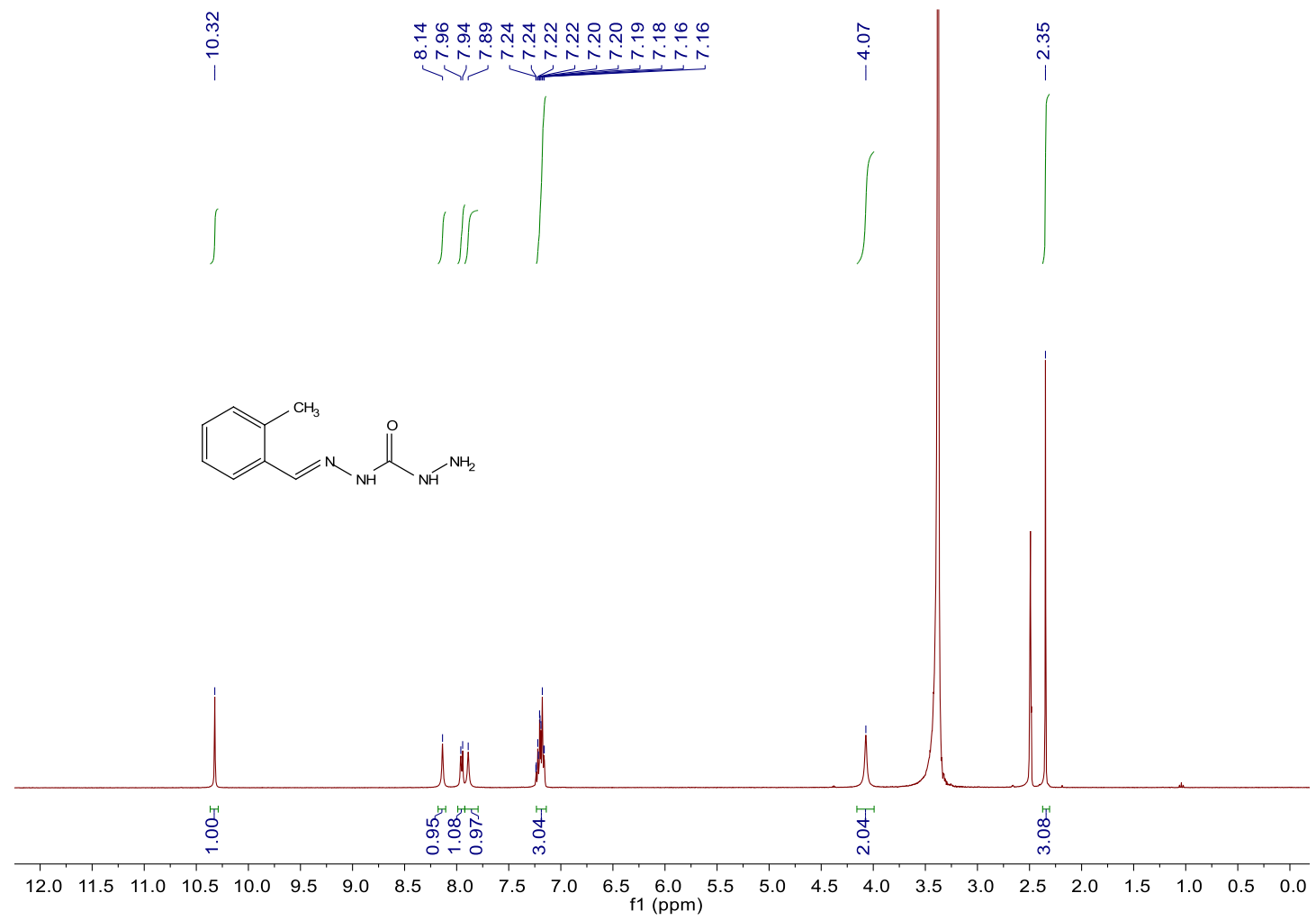

Figure S50. ${ }^{1} \mathrm{H}$ NMR spectrum (400 MHz, DMSO-d $6,25^{\circ} \mathrm{C}$ ) of $\mathbf{1 0 g}$.

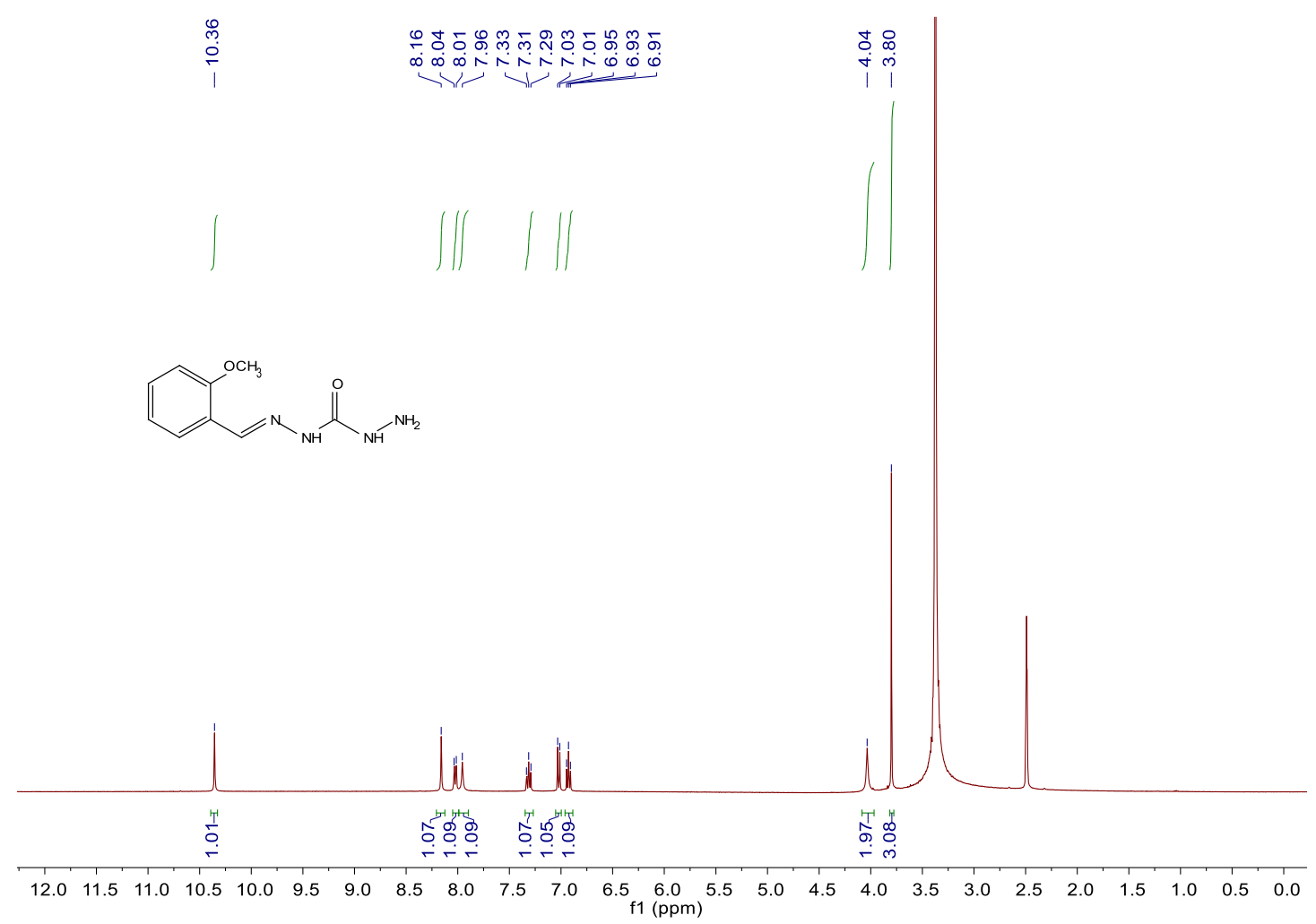

Figure S51. ${ }^{1} \mathrm{H}$ NMR spectrum $\left(400 \mathrm{MHz}, \mathrm{DMSO}_{-} \mathrm{d}_{6}, 25^{\circ} \mathrm{C}\right)$ of $\mathbf{1 0 h}$. 


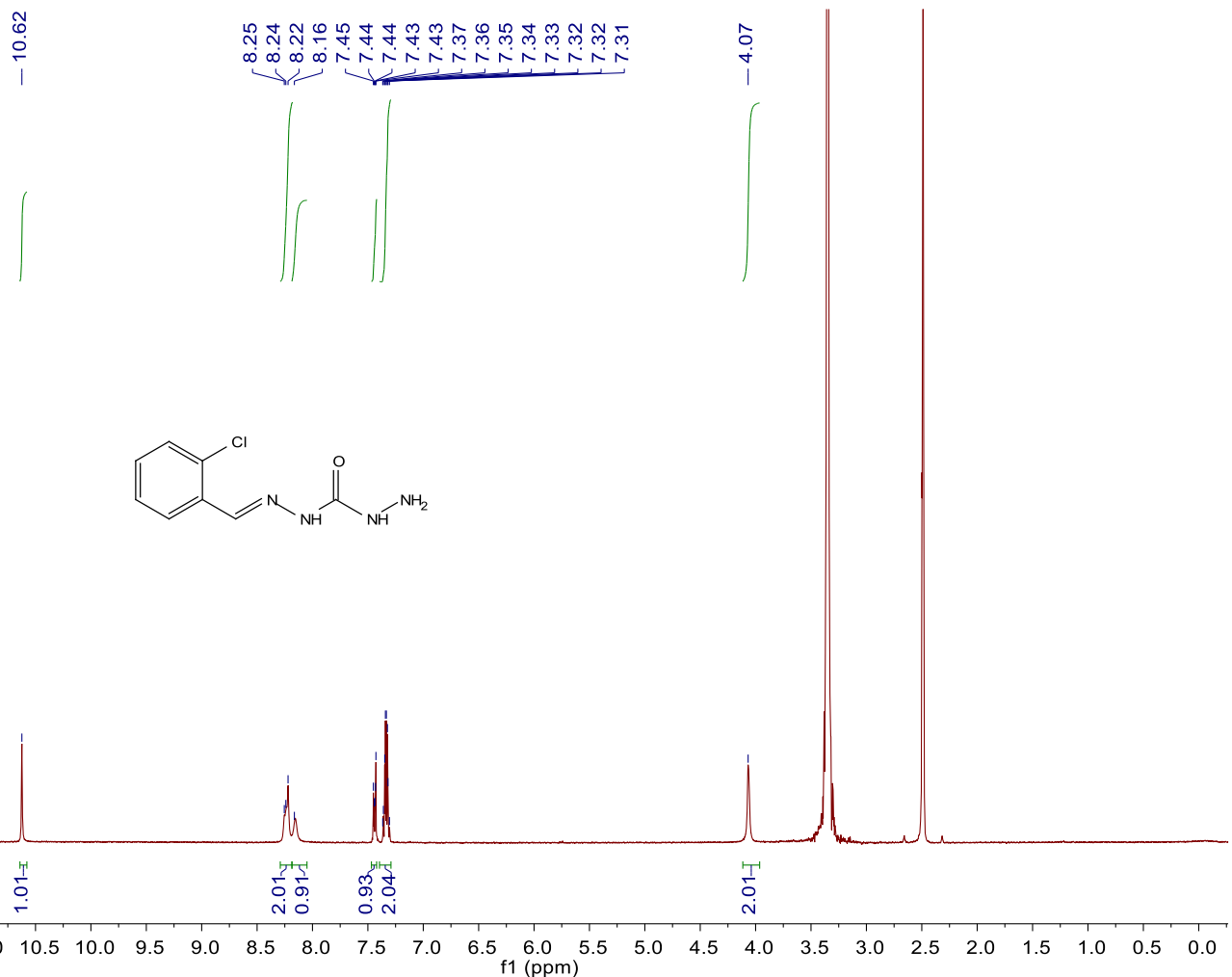

Figure S52. ${ }^{1} \mathrm{H}$ NMR spectrum $\left(400 \mathrm{MHz}, \mathrm{DMSO}-\mathrm{d}_{6}, 25^{\circ} \mathrm{C}\right)$ of $\mathbf{1 0 i}$.

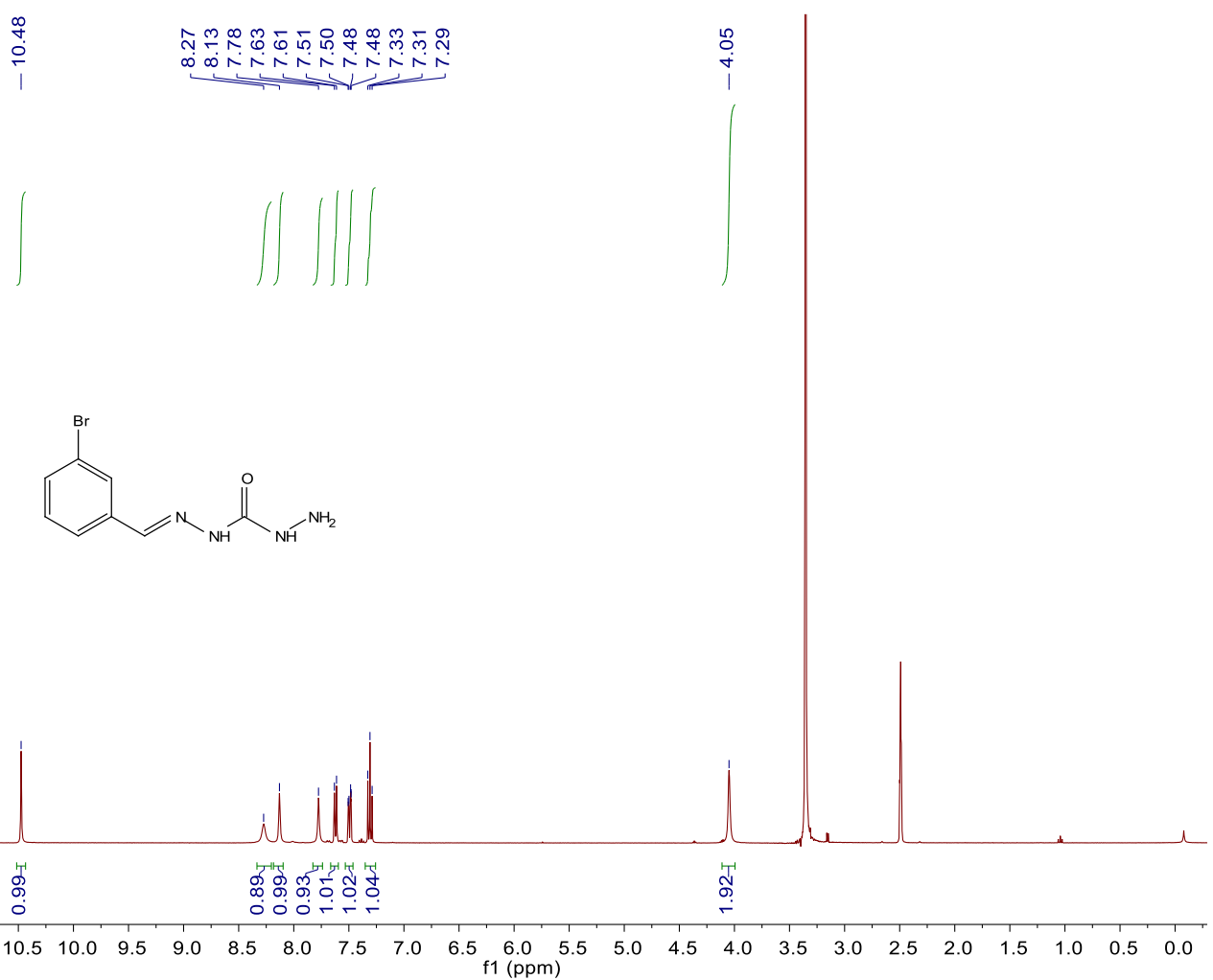

Figure S53. ${ }^{1} \mathrm{H}$ NMR spectrum (400 MHz, DMSO-d $6,25^{\circ} \mathrm{C}$ ) of 10j. 


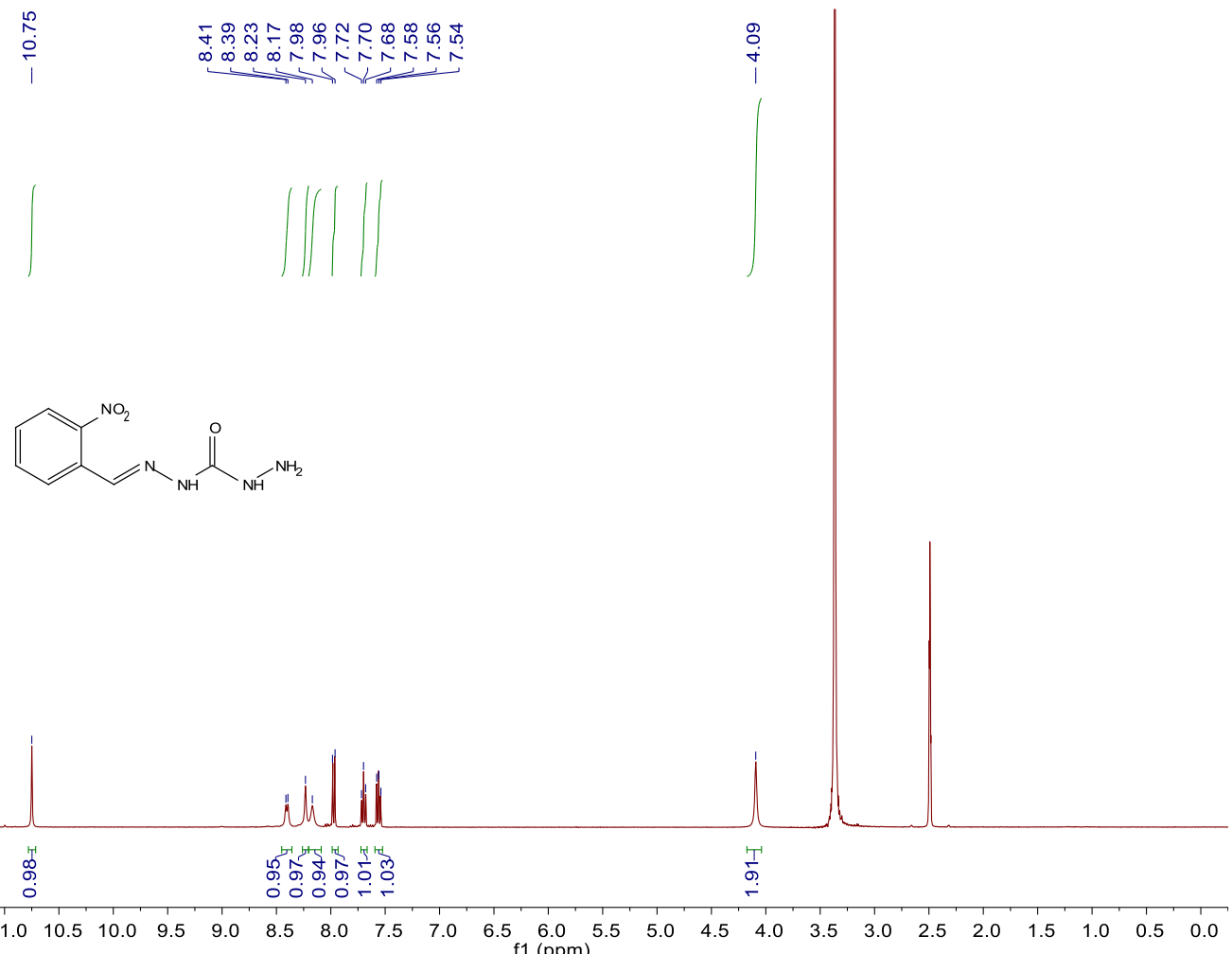

Figure S54. ${ }^{1} \mathrm{H}$ NMR spectrum (400 MHz, DMSO-d 6 , $25^{\circ} \mathrm{C}$ ) of $\mathbf{1 0 k}$. 
Table S1. The electronic energies and coordinates of the optimized structures

\begin{tabular}{|c|c|c|c|c|c|c|c|}
\hline \multicolumn{4}{|c|}{ Cage C1-1 } & $\mathrm{C}$ & -3.85155000 & 12.10963400 & 14.40933700 \\
\hline \multirow{2}{*}{\multicolumn{4}{|c|}{ E -4809.661455 a.u. (zero imaginary freq.) }} & $\mathrm{C}$ & -4.35715900 & 12.48550100 & 12.12785300 \\
\hline & & & & $\mathrm{C}$ & -5.71953300 & 12.58250100 & 12.41308300 \\
\hline $\mathrm{C}$ & -7.15132900 & 9.72296100 & -3.58991000 & $\mathrm{C}$ & -5.18945900 & 12.20161400 & 14.74204300 \\
\hline $\mathrm{C}$ & -8.07329500 & 7.38895800 & -2.29051000 & $\mathrm{C}$ & -6.67859700 & 12.82457300 & 11.33409800 \\
\hline $\mathrm{C}$ & -7.67320300 & 8.65147500 & -4.32466800 & $\mathrm{~N}$ & -6.24169000 & 13.00771400 & 10.13227800 \\
\hline $\mathrm{C}$ & -7.09959300 & 9.60562600 & -2.18821200 & $\mathrm{~N}$ & -7.19957200 & 13.20934100 & 9.14202300 \\
\hline $\mathrm{C}$ & -7.54753500 & 8.45482500 & -1.54538200 & $\mathrm{C}$ & -6.79507600 & 13.44870300 & 7.86768000 \\
\hline $\mathrm{C}$ & -8.13571300 & 7.49878000 & -3.68171700 & $\mathrm{C}$ & -7.07032600 & 12.22093800 & -3.47283800 \\
\hline $\mathrm{C}$ & -8.54123800 & 6.12320100 & -1.60999500 & $\mathrm{C}$ & -8.04335400 & 14.44190700 & -2.01725900 \\
\hline $\mathrm{C}$ & -11.99488900 & 6.87040600 & 0.80361600 & $\mathrm{C}$ & -8.43246200 & 12.57150700 & -3.51037100 \\
\hline $\mathrm{C}$ & -10.90645500 & 6.83518600 & -1.33712000 & $\mathrm{C}$ & -6.21221000 & 12.99046900 & -2.67615400 \\
\hline $\mathrm{C}$ & -9.70175200 & 6.17674700 & 0.59094700 & $\mathrm{C}$ & -6.69210700 & 14.09008600 & -1.95728800 \\
\hline $\mathrm{C}$ & -10.81266700 & 6.41357800 & 1.40216800 & $\mathrm{C}$ & -8.91257400 & 13.66821600 & -2.79951100 \\
\hline $\mathrm{C}$ & -12.03355300 & 7.08037900 & -0.57607600 & $\mathrm{C}$ & -8.56239700 & 15.63514100 & -1.24713200 \\
\hline $\mathrm{C}$ & -10.73648700 & 6.19168700 & 2.84614500 & $\mathrm{C}$ & -10.30051200 & 14.36213600 & 2.45270200 \\
\hline $\mathrm{N}$ & -9.63150700 & 5.77640300 & 3.37146500 & $\mathrm{C}$ & -10.52632900 & 14.99004900 & 0.14726000 \\
\hline $\mathrm{N}$ & -9.63355500 & 5.59464300 & 4.75229300 & $\mathrm{C}$ & -8.38085200 & 14.99494100 & 1.14696100 \\
\hline $\mathrm{C}$ & -8.50798600 & 5.15480000 & 5.37181900 & $\mathrm{C}$ & -8.91317300 & 14.57623200 & 2.36186400 \\
\hline $\mathrm{C}$ & -0.93187200 & 5.61685700 & 13.44917100 & $\mathrm{C}$ & -11.10287700 & 14.56794200 & 1.33602700 \\
\hline $\mathrm{C}$ & -2.86955900 & 3.56317200 & 13.32714100 & $\mathrm{C}$ & -8.01531600 & 14.38098500 & 3.49851000 \\
\hline $\mathrm{C}$ & -2.06942800 & 5.64319200 & 14.27979700 & $\mathrm{~N}$ & -8.50465700 & 14.07390700 & 4.65453300 \\
\hline $\mathrm{C}$ & -0.78997900 & 4.54995900 & 12.55598600 & $\mathrm{~N}$ & -7.57474100 & 13.89277500 & 5.67735500 \\
\hline $\mathrm{C}$ & -1.75074300 & 3.53408100 & 12.49223000 & $\mathrm{C}$ & -7.99619100 & 13.62047800 & 6.93923600 \\
\hline $\mathrm{C}$ & -3.02275000 & 4.63177800 & 14.22364100 & $\mathrm{~N}$ & -9.76534300 & 6.38221300 & -0.74183200 \\
\hline $\mathrm{C}$ & -3.88603300 & 2.44430800 & 13.28524000 & $\mathrm{O}$ & -7.42040200 & 4.88139900 & 4.83836900 \\
\hline $\mathrm{C}$ & -7.58431300 & 3.87015800 & 11.67475100 & $\mathrm{O}$ & -9.82796500 & 5.30207800 & 7.40875300 \\
\hline $\mathrm{C}$ & -6.27308700 & 3.11874000 & 13.53886100 & $\mathrm{~N}$ & -5.21087600 & 2.92931900 & 12.71401200 \\
\hline $\mathrm{C}$ & -5.29259000 & 3.19651200 & 11.38578800 & $\mathrm{~N}$ & -3.46023600 & 12.25727300 & 13.11043600 \\
\hline $\mathrm{C}$ & -6.47647300 & 3.66758300 & 10.83010600 & $\mathrm{~N}$ & -9.18555800 & 15.19950200 & 0.07440400 \\
\hline $\mathrm{C}$ & -7.47422800 & 3.59304300 & 13.03173800 & $\mathrm{~N}$ & 0.03018200 & 11.38492800 & -3.84551900 \\
\hline $\mathrm{C}$ & -6.53410300 & 3.92521900 & 9.39165700 & $\mathrm{~N}$ & 5.05440000 & 7.34367800 & 9.28171700 \\
\hline $\mathrm{N}$ & -7.63032700 & 4.36961200 & 8.87185200 & $\mathrm{O}$ & 3.69332300 & 11.95546300 & 2.60653800 \\
\hline $\mathrm{N}$ & -7.61912900 & 4.57546000 & 7.49406700 & $\mathrm{O}$ & 2.71429400 & 8.70679700 & 3.64033800 \\
\hline $\mathrm{C}$ & -8.74131200 & 5.02503800 & 6.87543700 & $\mathrm{O}$ & -9.16918500 & 13.51684700 & 7.33307700 \\
\hline $\mathrm{C}$ & -6.58866800 & 10.98861700 & -4.27799400 & $\mathrm{O}$ & -5.62394000 & 13.52948800 & 7.46144400 \\
\hline $\mathrm{C}$ & -7.10431800 & 11.10632400 & -5.74609700 & $\mathrm{H}$ & -7.72522800 & 8.69892200 & -5.40390000 \\
\hline $\mathrm{C}$ & 0.14990300 & 6.71101700 & 13.61933800 & $\mathrm{H}$ & -6.69893900 & 10.42254500 & -1.60051900 \\
\hline $\mathrm{C}$ & 0.71512700 & 6.54636300 & 15.06317600 & $\mathrm{H}$ & -7.49150600 & 8.38199300 & -0.46433900 \\
\hline $\mathrm{C}$ & 1.27154600 & 6.53966200 & 12.56304100 & $\mathrm{H}$ & -8.53761700 & 6.68021100 & -4.26870400 \\
\hline $\mathrm{C}$ & 3.27523200 & 6.19336400 & 10.59577700 & $\mathrm{H}$ & -7.78102100 & 5.71113700 & -0.94497900 \\
\hline $\mathrm{C}$ & 2.47391500 & 5.88623300 & 12.86635100 & $\mathrm{H}$ & -8.83161800 & 5.36888700 & -2.34252100 \\
\hline $\mathrm{C}$ & 1.08690700 & 7.00553800 & 11.24846400 & $\mathrm{H}$ & -12.87027500 & 7.05921900 & 1.41268600 \\
\hline $\mathrm{C}$ & 2.06930100 & 6.83277100 & 10.27728400 & $\mathrm{H}$ & -10.87572500 & 6.98614900 & -2.40594800 \\
\hline $\mathrm{C}$ & 3.46756600 & 5.72040500 & 11.89633800 & $\mathrm{H}$ & -8.76918300 & 5.82220100 & 1.00444800 \\
\hline $\mathrm{C}$ & 4.34906300 & 6.01908700 & 9.54561000 & $\mathrm{H}$ & -12.93173400 & 7.43241800 & -1.06258700 \\
\hline $\mathrm{C}$ & 6.26648100 & 9.78617600 & 8.88038000 & $\mathrm{H}$ & -11.64008800 & 6.39580000 & 3.41903800 \\
\hline $\mathrm{C}$ & 5.90263900 & 7.83406300 & 10.23052700 & $\mathrm{H}$ & -10.46843600 & 5.78044200 & 5.32100700 \\
\hline $\mathrm{C}$ & 4.79430000 & 8.03081600 & 8.14906000 & $\mathrm{H}$ & -2.20597800 & 6.46101200 & 14.97714600 \\
\hline $\mathrm{C}$ & 5.38910300 & 9.27136300 & 7.91589500 & $\mathrm{H}$ & 0.07414500 & 4.50219300 & 11.90782900 \\
\hline $\mathrm{C}$ & 6.52190800 & 9.05524800 & 10.04180900 & $\mathrm{H}$ & -1.61854700 & 2.71123500 & 11.79801600 \\
\hline $\mathrm{C}$ & 5.09689600 & 10.01652500 & 6.69150100 & $\mathrm{H}$ & -3.88633000 & 4.66779600 & 14.87940700 \\
\hline $\mathrm{N}$ & 4.30684200 & 9.50943900 & 5.80358200 & $\mathrm{H}$ & -4.11118900 & 2.06412100 & 14.28251100 \\
\hline $\mathrm{N}$ & 4.07078500 & 10.29050100 & 4.67379500 & $\mathrm{H}$ & -3.54971900 & 1.62532000 & 12.64828100 \\
\hline $\mathrm{C}$ & 3.27644100 & 9.81359200 & 3.68106700 & $\mathrm{H}$ & -8.51059000 & 4.23664200 & 11.25345800 \\
\hline $\mathrm{C}$ & -5.04296100 & 10.91485700 & -4.35829200 & $\mathrm{H}$ & -6.13540900 & 2.87814500 & 14.58246100 \\
\hline $\mathrm{C}$ & -2.23960500 & 10.76070300 & -4.71225200 & $\mathrm{H}$ & -4.40569500 & 3.02485700 & 10.79137300 \\
\hline $\mathrm{C}$ & -4.33202900 & 11.95756700 & -4.98366000 & $\mathrm{H}$ & -8.30820900 & 3.73578700 & 13.70363500 \\
\hline $\mathrm{C}$ & -4.31983700 & 9.80308700 & -3.90784600 & $\mathrm{H}$ & -5.63332200 & 3.71886000 & 8.81461100 \\
\hline $\mathrm{C}$ & -2.93447700 & 9.72558400 & -4.08184300 & $\mathrm{H}$ & -6.78639200 & 4.38296700 & 6.92449500 \\
\hline $\mathrm{C}$ & -2.95259900 & 11.88340600 & -5.15794000 & $\mathrm{H}$ & -6.80109600 & 12.06983500 & -6.16269300 \\
\hline $\mathrm{C}$ & -0.74730600 & 10.65960200 & -4.93695100 & $\mathrm{H}$ & -8.19400800 & 11.03324900 & -5.78794200 \\
\hline $\mathrm{C}$ & 1.41648100 & 12.69996000 & -1.85873500 & $\mathrm{H}$ & -6.67338100 & 10.31318100 & -6.36323600 \\
\hline $\mathrm{C}$ & 0.37283200 & 12.68847600 & -4.02024500 & $\mathrm{H}$ & 1.59137200 & 7.17985900 & 15.22269000 \\
\hline $\mathrm{C}$ & 0.34704200 & 10.71939300 & -2.70741700 & $\mathrm{H}$ & 1.00023400 & 5.50569800 & 15.23902600 \\
\hline $\mathrm{C}$ & 1.04992600 & 11.35277900 & -1.68739600 & $\mathrm{H}$ & -0.05527800 & 6.81865300 & 15.78927000 \\
\hline $\mathrm{C}$ & 1.06857800 & 13.36437600 & -3.02974900 & $\mathrm{H}$ & 2.65157600 & 5.49755200 & 13.85976100 \\
\hline $\mathrm{C}$ & 1.38754100 & 10.59352300 & -0.48427700 & $\mathrm{H}$ & 0.16777600 & 7.51257200 & 10.98541200 \\
\hline $\mathrm{N}$ & 2.10483500 & 11.14450900 & 0.43875200 & $\mathrm{H}$ & 1.90239900 & 7.19916900 & 9.26987300 \\
\hline $\mathrm{N}$ & 2.37029300 & 10.35397200 & 1.55634200 & $\mathrm{H}$ & 4.39063400 & 5.21303200 & 12.15471600 \\
\hline $\mathrm{C}$ & 3.14665900 & 10.84144500 & 2.55794700 & $\mathrm{H}$ & 3.93309600 & 5.70038600 & 8.58928500 \\
\hline $\mathrm{C}$ & -0.45961200 & 8.12281700 & 13.44232700 & $\mathrm{H}$ & 5.11653200 & 5.31497400 & 9.86932400 \\
\hline $\mathrm{C}$ & -1.48250100 & 10.72628200 & 13.02358400 & $\mathrm{H}$ & 6.74119300 & 10.74638300 & 8.71969600 \\
\hline $\mathrm{C}$ & 0.15510200 & 9.24130600 & 14.02361000 & $\mathrm{H}$ & 6.05226900 & 7.22860500 & 11.11213900 \\
\hline $\mathrm{C}$ & -1.59682600 & 8.33457200 & 12.64371300 & $\mathrm{H}$ & 4.11522100 & 7.59022300 & 7.43422800 \\
\hline $\mathrm{C}$ & -2.09689100 & 9.61828600 & 12.42754600 & $\mathrm{H}$ & 7.19571100 & 9.42748100 & 10.79993900 \\
\hline $\mathrm{C}$ & -0.35655000 & 10.52585000 & 13.82830100 & $\mathrm{H}$ & 5.57252300 & 10.99063000 & 6.58831200 \\
\hline $\mathrm{C}$ & -1.98738900 & 12.13059500 & 12.76919200 & $\mathrm{H}$ & 4.48134500 & 11.22629300 & 4.57030600 \\
\hline
\end{tabular}




$\begin{array}{lcc}-4.86116200 & 12.83169100 & -5.34345200 \\ -4.83657700 & 8.98402800 & -3.42772800 \\ -2.39929800 & 8.84600000 & -3.74072200 \\ -2.42840500 & 12.69184300 & -5.65600300 \\ -0.45026900 & 11.12429100 & -5.87759400 \\ -0.40980000 & 9.62332900 & -4.91457200 \\ 1.96453600 & 13.20442900 & -1.07460500 \\ 0.08572000 & 13.15099300 & -4.95306600 \\ 0.03934300 & 9.68536800 & -2.63770900 \\ 1.33560100 & 14.39935800 & -3.18774600 \\ 1.01248800 & 9.57278600 & -0.42210900 \\ 1.98957300 & 9.40438200 & 1.64731000 \\ 1.03997600 & 9.12293600 & 14.63515400 \\ -2.09293500 & 7.48982500 & 12.18372400 \\ -2.96885700 & 9.75443100 & 11.79679600 \\ 0.12884000 & 11.37525400 & 14.29618000 \\ -1.89762300 & 12.40796100 & 11.71740600 \\ -1.45548900 & 12.85883700 & 13.38333200 \\ -7.18865100 & 12.50900400 & 13.98999600 \\ -3.07486800 & 11.92292900 & 15.13628900 \\ -3.98395400 & 12.59028800 & 11.11993900 \\ -5.48502400 & 12.08901300 & 15.77513000 \\ -7.73298000 & 12.83337400 & 11.60531500 \\ -8.20492200 & 13.18100400 & 9.34919800 \\ -9.12634000 & 11.98411600 & -4.09871100 \\ -5.16104700 & 12.74178800 & -2.62205500 \\ -6.00803000 & 14.67931900 & -1.35620800 \\ -9.96427400 & 13.92766600 & -2.85464100 \\ -9.34685100 & 16.16120800 & -1.79183900 \\ -7.76067800 & 16.32959100 & -0.99493000 \\ -10.72582300 & 14.03916400 & 3.39335200 \\ -11.10215400 & 15.17231400 & -0.74811400 \\ -7.32343800 & 15.17860700 & 1.01353600 \\ -12.17088800 & 14.40943300 & 1.37543700 \\ -6.94952700 & 14.50935900 & 3.31366100 \\ -6.56444800 & 13.96295000 & 5.50468800 \\ & & \end{array}$

Cage C1-2

E -4809.660632 a.u. (zero imaginary freq.)

$\begin{array}{lcrr}\text { E - 4809.660632 a.u. (zero imaginary freq.) } & \\ \text { C } & -6.49201800 & 9.77755500 & -2.58356100 \\ \text { C } & -5.41750600 & 7.16379100 & -2.66941400 \\ \text { C } & -6.96538900 & 8.83277800 & -3.50861500 \\ \text { C } & -5.47537100 & 9.38560300 & -1.69937500 \\ \text { C } & -4.94709400 & 8.09396500 & -1.73630600 \\ \text { C } & -6.43067400 & 7.54545600 & -3.55710600 \\ \text { C } & -4.82609300 & 5.77268600 & -2.72378300 \\ \text { C } & -7.75859100 & 2.93674300 & -1.44076900 \\ \text { C } & -6.45998100 & 3.94321500 & -3.19032200 \\ \text { C } & -6.15771800 & 4.66480800 & -0.95531400 \\ \text { C } & -7.11695800 & 3.76219100 & -0.50257500 \\ \text { C } & -7.42363600 & 3.03377200 & -2.78934400 \\ \text { C } & -7.45604100 & 3.67160900 & 0.91699400 \\ \text { N } & -6.87195600 & 4.43748400 & 1.77868300 \\ \text { N } & -7.27000400 & 4.26798000 & 3.10485100 \\ \text { C } & -6.71247200 & 5.02356100 & 4.08525900 \\ \text { C } & -1.67468400 & 7.44334600 & 12.86404100 \\ \text { C } & -4.48172200 & 7.29049900 & 13.16555100 \\ \text { C } & -2.48587400 & 8.55180300 & 12.59903500 \\ \text { C } & -2.29643500 & 6.24613100 & 13.27180900 \\ \text { C } & -3.67702000 & 6.16937000 & 13.42394200 \\ \text { C } & -3.87534100 & 8.47682100 & 12.74584500 \\ \text { C } & -5.97383000 & 7.23381500 & 13.38316800 \\ \text { C } & -8.02660700 & 4.88745400 & 10.44415500 \\ \text { C } & -7.67806200 & 5.53934300 & 12.72678800 \\ \text { C } & -6.33970800 & 6.50032700 & 11.02875400 \\ \text { C } & -6.99823000 & 5.76309200 & 10.05050700 \\ \text { C } & -8.36230700 & 4.78164000 & 11.78846100 \\ \text { C } & -6.59914600 & 5.91681600 & 8.65133800 \\ \text { N } & -7.18182600 & 5.20107300 & 7.74723400 \\ \text { N } & -6.75829800 & 5.39511700 & 6.43355800 \\ \text { C } & -7.31280100 & 4.65251400 & 5.44094800 \\ \text { C } & -7.10681700 & 11.19416600 & -2.49374700 \\ \text { C } & -7.69941400 & 11.62821800 & -3.87060900 \\ \text { C } & -0.12838000 & 7.50747000 & 12.80128600 \\ \text { C } & 0.38653100 & 7.19934200 & 14.24150700 \\ \text { C } & 0.42585200 & 6.45569000 & 11.81064900 \\ \text { C } & 1.48468000 & 4.56397700 & 9.99445400 \\ \text { C } & 1.75749600 & 6.01598800 & 11.91717200 \\ \text { C } & -0.35434200 & 5.94729400 & 10.76280300 \\ \text { C } & 0.17010500 & 5.01792300 & 9.85957200\end{array}$

\begin{tabular}{|c|c|c|}
\hline 764500 & 5.07608300 & 038000 \\
\hline 2.01247700 & 3.49068800 & 9.06612500 \\
\hline 5.57918500 & 4.76326900 & 7.08716400 \\
\hline 47513800 & 3.36784400 & 8.69878100 \\
\hline 0269700 & 4.90932100 & 7.42907500 \\
\hline 4.34338200 & 5.34581000 & 6.75997200 \\
\hline 5.63797900 & 3.77415400 & 8.06661200 \\
\hline 4.25860200 & 6.37831400 & 5.72718700 \\
\hline 3.13053800 & 6.95934700 & 5.48431500 \\
\hline 3.13724400 & 7.90743400 & 4.46204000 \\
\hline 1.99808100 & 8.58342100 & 4.16520600 \\
\hline-5.98918800 & 12.19035600 & -2.09616200 \\
\hline-3.89949700 & 13.97580400 & -1.43663300 \\
\hline-4.96115400 & 12.47319700 & -3.01537100 \\
\hline-5.93865700 & 12.80360900 & -0.83889200 \\
\hline-4.90524800 & 13.68794300 & -0.51156400 \\
\hline-3.93428100 & 13.35660800 & -2.69505600 \\
\hline-2.79729700 & 14.94919300 & -1.08843700 \\
\hline 0.92034400 & 12.88589100 & -0.60479700 \\
\hline-0.45706900 & 14.44771400 & -1.79868200 \\
\hline-1.32783100 & 13.35542700 & 0.11134200 \\
\hline-0.13732200 & 12.66289200 & 0.29705300 \\
\hline 0.75139800 & 13.78070100 & -1.65 \\
\hline-0.03116400 & 11.72683700 & 1.41529400 \\
\hline 1.08019600 & 11.10106900 & 1.62148300 \\
\hline 1.09180100 & 3200 & 6400 \\
\hline 2.22819100 & 9.52722200 & 2.98569200 \\
\hline 0.33508900 & 8.91041500 & 12.33747900 \\
\hline 1.09334700 & 11.49 & 11.47150900 \\
\hline 0.70868500 & 9.90131100 & 0500 \\
\hline 0.34503100 & 9.24217 & 1800 \\
\hline 0.71300900 & 10.5 & 7200 \\
\hline 1.09039300 & 11.17347000 & 9100 \\
\hline 1.52262200 & 12.86855600 & 11.00384800 \\
\hline-1.31789800 & 15.76642100 & 12.35205000 \\
\hline 0.84165200 & 14.73297000 & 12.51757900 \\
\hline-0.67008300 & 14.00520000 & 10.84675200 \\
\hline-1.62453000 & 14.92828800 & 21700 \\
\hline-0.07661200 & 15.66229300 & 5200 \\
\hline-2.92291600 & 15.03081100 & 37400 \\
\hline-3.21521300 & 14.25964800 & 9.60838500 \\
\hline-4.48144100 & 14.45063900 & 9.05382400 \\
\hline-4.88677900 & 13.69816800 & 8.00001700 \\
\hline-8.25101200 & 11.21063000 & -1.45010300 \\
\hline-10.34477600 & 11.31718300 & 0.44874400 \\
\hline-8.51560500 & 10.12347200 & -0.60946000 \\
\hline-9.06394400 & 12.35510900 & -1.32959000 \\
\hline-10.09510400 & 12.40889000 & -0.39677700 \\
\hline-9.54989300 & 10.17461500 & 0.33125500 \\
\hline-11.45885400 & 11.37402000 & 1.46636300 \\
\hline-10.50765600 & 14.00432500 & 4.71482000 \\
\hline-12.01137800 & 13.27694900 & 2.99089500 \\
\hline-9.92709400 & 12.18779700 & 3.24778300 \\
\hline-9.58961600 & 13.01758700 & 4.31079900 \\
\hline-11.72016900 & 14.12798800 & 4.04727000 \\
\hline-8.29115400 & 12.84015200 & 4.95870800 \\
\hline-7.96535700 & 13.59488400 & 45700 \\
\hline-6.70560600 & 13.36712100 & 6.50880900 \\
\hline-6.29278900 & 14.11240500 & 7.56548500 \\
\hline-5.84555500 & 4.73743000 & -2.26994300 \\
\hline-5.84141200 & 5.89906800 & 3.95448300 \\
\hline-8.18771000 & 3.77952700 & 5.56395700 \\
\hline-6.68439900 & 6.37935500 & 12.33444500 \\
\hline 0.53121200 & 13.92 & 11.46 \\
\hline-11.11820200 & 12.32496500 & 2.61382800 \\
\hline-1.46718400 & 14.22813300 & -0.91843000 \\
\hline 3.28282100 & 3.93742600 & 8.36724800 \\
\hline 3.32308900 & 9.61653800 & 2.40648200 \\
\hline 0.89924600 & 8.48301200 & 4.73558100 \\
\hline-6.92651300 & 15.01528400 & 8.13637900 \\
\hline-4.24963900 & 12.79618400 & 7.43136600 \\
\hline-7.75317400 & 9.09411300 & -4.20247400 \\
\hline-5.09351500 & 10.09445900 & -0.97565100 \\
\hline-4.16056700 & 7.81222000 & -1.04480900 \\
\hline-6.80566000 & 6.83579800 & -4.28703000 \\
\hline-3.96767000 & 5.67922500 & -2.05726900 \\
\hline-4.53591300 & 5.49046300 & -3.73644200 \\
\hline-8.50894200 & 2.22944600 & -1.11001200 \\
\hline-6.15692600 & 4.05614200 & -4.22034400 \\
\hline-5.63895900 & 5.32971700 & -0.28012700 \\
\hline
\end{tabular}




\begin{tabular}{|c|c|c|}
\hline-7.90019500 & 2.41042600 & -3.53204000 \\
\hline-8.21178100 & 2.93512700 & 1.18552600 \\
\hline-7.98325000 & 3.57624200 & 3.36636100 \\
\hline-2.03792000 & 9.48334600 & 12.28254200 \\
\hline-1.69381700 & 5.36959500 & 13.47666600 \\
\hline-4.13313400 & 5.24231200 & 13.75376800 \\
\hline-4.48544600 & 9.35094100 & 12.54582800 \\
\hline-6.41786200 & 8.22918800 & 13.32275700 \\
\hline-6.22052000 & 6.78216800 & 14.34475600 \\
\hline-8.54361400 & 4.30601500 & 9.69294700 \\
\hline-7.89865600 & 5.49425200 & 13.78328900 \\
\hline-5.53805000 & 7.18508900 & 10.79086000 \\
\hline-9.14663500 & 4.11696800 & 12.12076600 \\
\hline-5.81305800 & 6.63839900 & 8.43454600 \\
\hline-6.03504800 & 6.08384000 & 6.19432200 \\
\hline-7.97401500 & 12.68536200 & -3.84006600 \\
\hline-8.59537100 & 11.04733200 & -4.10510100 \\
\hline-6.96701000 & 11.48280500 & -4.66885000 \\
\hline 1.46243200 & 7.37421000 & 4500 \\
\hline 0.17952700 & 0100 & 51000 \\
\hline-0.12647900 & 39600 & 19700 \\
\hline 2.39702700 & 6.40149200 & 12.70073600 \\
\hline-1.38027900 & 60300 & 068200 \\
\hline 1000 & 56600 & $\$ 7100$ \\
\hline 3.30 & 5300 & 11.1 \\
\hline $1.28^{\circ}$ & 3.25 & 800 \\
\hline 2.27139800 & 2.58030700 & 6500 \\
\hline 6.47780300 & 5.08 & 38400 \\
\hline 4.457 & 2.5 & 900 \\
\hline 2.226 & 5.32 & 6000 \\
\hline 6.57 & 3.31 & 300 \\
\hline 5.18 & 6.60 & 400 \\
\hline 3.98 & 900 & 5400 \\
\hline-4.96 & 0800 & 5000 \\
\hline-6.70869100 & 12.59757100 & 40700 \\
\hline-4.88929100 & 14.15825400 & 0.46550500 \\
\hline-3.15611900 & 13.56697100 & -3.42107700 \\
\hline-2.9 & 7800 & 00 \\
\hline-2.64 & 4700 & 7400 \\
\hline 1.85 & 6000 & 0400 \\
\hline-0.64101400 & 3800 & 96000 \\
\hline-2.17681000 & 13.22670600 & 0.76874700 \\
\hline 1.54375500 & 13.96971000 & -2.36385500 \\
\hline-0.91841300 & 11.59603000 & 357400 \\
\hline 0.254 & 10.0 & \\
\hline 0.708 & 100 & 64700 \\
\hline 0.06619000 & 200 & 24200 \\
\hline 0.71491100 & 10.75142700 & 4700 \\
\hline 1.38546900 & 11.91766500 & 13.56580600 \\
\hline 1.55910200 & 12.92603300 & 9.91531000 \\
\hline 2.48 & & 11.4 \\
\hline-2.04770000 & 16.4 & 30600 \\
\hline 1.82078900 & 14.616 & 12.95763500 \\
\hline-0.85381300 & 13.32899700 & 10.02458000 \\
\hline 0.18603100 & 16.29569200 & 13.81011000 \\
\hline-3.60605700 & 15.78178900 & 10.99858300 \\
\hline-5.12255900 & 15.16541100 & \\
\hline-7.91207700 & 9.2 & -0.6 \\
\hline-8.88424100 & 13.21569900 & -1.96164800 \\
\hline-10.70908900 & 13.29991700 & -0.32248100 \\
\hline-9.73804300 & 9.31965800 & 0.97158600 \\
\hline-11.63450800 & 10.39615500 & 1.91796700 \\
\hline-12.38632900 & 11.74975200 & 1.03334600 \\
\hline-10.25558200 & 14.65876600 & 5.53817400 \\
\hline-12.93657100 & 13.33262200 & 2.43687200 \\
\hline-9.26039000 & 11.41630600 & 2.88918400 \\
\hline-12.44313000 & 14.87923300 & 4.33027800 \\
\hline-7.64177500 & 12.06319100 & 4.55685900 \\
\hline-6.07461300 & 12.64713200 & 6.13696100 \\
\hline
\end{tabular}

\begin{tabular}{|c|c|c|c|}
\hline & .14974200 & -7.47067100 & 6.70719500 \\
\hline $\mathrm{C}$ & -0.80713900 & -6.33783700 & 4.85958500 \\
\hline C & -0.27782900 & -7.27416300 & 3.97255900 \\
\hline $\mathrm{C}$ & 0.69963200 & -8.42033600 & 5.86659500 \\
\hline $\mathrm{C}$ & -0.53352600 & -7.16619700 & 2.53579600 \\
\hline $\mathrm{N}$ & -1.27608700 & -6.20906200 & 2.08670900 \\
\hline $\mathrm{N}$ & -1.49174000 & -6.18978000 & 0.71064800 \\
\hline $\mathrm{C}$ & -2.28351600 & -5.22778700 & 0.16983500 \\
\hline $\mathrm{C}$ & -4.95222800 & 2.02412800 & -8.76331400 \\
\hline $\mathrm{C}$ & -6.24013000 & -0.31571000 & -7.82945600 \\
\hline $\mathrm{C}$ & -5.19203700 & 0.93887600 & -9.62248200 \\
\hline $\mathrm{C}$ & -5.35690300 & 1.90774400 & -7.42430000 \\
\hline $\mathrm{C}$ & -5.99042400 & 0.75213800 & -6.96121800 \\
\hline $\mathrm{C}$ & -5.83363400 & -0.21160500 & -9.16576500 \\
\hline $\mathrm{C}$ & -6.94496200 & -1.55980800 & -7.33675400 \\
\hline $\mathrm{C}$ & -4.08814700 & -4.46834400 & -6.03216100 \\
\hline $\mathrm{C}$ & -5.40052000 & -3.46233100 & -7.77092100 \\
\hline $\mathrm{C}$ & -5.58188700 & -2.64301400 & -5.55765800 \\
\hline $\mathrm{C}$ & -4.65232500 & -3.57449400 & -5.10472000 \\
\hline $\mathrm{C}$ & -4.46641800 & -4.40383600 & -7.36869300 \\
\hline $\mathrm{C}$ & -4.31247900 & -3.60435600 & -3.68252200 \\
\hline $\mathrm{N}$ & -3.50165700 & -4.50933600 & -3.24294200 \\
\hline $\mathrm{N}$ & -3.23288900 & -4.48606900 & -1.87547700 \\
\hline $\mathrm{C}$ & -2.42168700 & -5.43332400 & -1.33752400 \\
\hline $\mathrm{C}$ & -4.20944600 & 3.27796000 & -9.28639500 \\
\hline $\mathrm{C}$ & -4.70631100 & 3.62975300 & -10.72306000 \\
\hline $\mathrm{C}$ & 2.61403300 & -1.13072100 & 8.64339500 \\
\hline $\mathrm{C}$ & 2.94675900 & -1.44669600 & 10.13180600 \\
\hline $\mathrm{C}$ & 3.89603800 & -1.11342800 & 7.77392400 \\
\hline $\mathrm{C}$ & 6.19081400 & -1.12393700 & 6.11801300 \\
\hline $\mathrm{C}$ & 5.11488000 & -1.62537200 & 8.23564600 \\
\hline $\mathrm{C}$ & 3.84753800 & -0.60752000 & 6.46130100 \\
\hline $\mathrm{C}$ & 4.97222400 & -0.61763200 & 5.64270200 \\
\hline $\mathrm{C}$ & 6.25263200 & -1.62315200 & 7.42052300 \\
\hline $\mathrm{C}$ & 7.41319200 & -1.15253800 & 5.22573400 \\
\hline $\mathrm{C}$ & 8.69082300 & 2.86675500 & 4.52633600 \\
\hline $\mathrm{C}$ & 8.61034200 & 0.91279000 & 5.92137200 \\
\hline $\mathrm{C}$ & 7.59322900 & 0.86062500 & 3.78428200 \\
\hline $\mathrm{C}$ & 7.96817800 & 2.18175500 & 3.53956800 \\
\hline $\mathrm{C}$ & 9.01551800 & 2.21887700 & 5.71970800 \\
\hline $\mathrm{C}$ & 7.59817800 & 2.83546300 & 2.28423200 \\
\hline $\mathrm{N}$ & 6.94570000 & 2.17207200 & 1.38796400 \\
\hline $\mathrm{N}$ & 6.60559200 & 2.86414000 & 0.22794300 \\
\hline $\mathrm{C}$ & 5.92495300 & 2.21974800 & -0.75454100 \\
\hline $\mathrm{C}$ & -2.69210800 & 2.96598800 & -9.28488800 \\
\hline $\mathrm{C}$ & 0.07741300 & 2.39238900 & -9.23743400 \\
\hline $\mathrm{C}$ & -1.85736600 & 3.35785000 & -10.33988800 \\
\hline $\mathrm{C}$ & -2.10917100 & 2.29368100 & -8.19498600 \\
\hline $\mathrm{C}$ & -0.74638000 & 2.00867500 & -8.16963200 \\
\hline $\mathrm{C}$ & -0.48842800 & 3.07306700 & -10.31815200 \\
\hline $\mathrm{C}$ & 1.55051400 & 2.05182300 & -9.23414500 \\
\hline $\mathrm{C}$ & 3.63569300 & 4.51087000 & -6.42141900 \\
\hline $\mathrm{C}$ & 2.48920900 & 4.23665600 & -8.51328900 \\
\hline $\mathrm{C}$ & 2.76449100 & 2.36971200 & -7.08520200 \\
\hline $\mathrm{C}$ & 3.43734600 & 3.14744700 & -6.14660900 \\
\hline $\mathrm{C}$ & 3.15756700 & 5.04983300 & -7.61271600 \\
\hline $\mathrm{C}$ & 3.90509300 & 2.51065200 & -4.91731500 \\
\hline $\mathrm{N}$ & 4.53904600 & 3.20747500 & -4.03279100 \\
\hline $\mathrm{N}$ & 4.93663800 & 2.51754300 & -2.88805800 \\
\hline $\mathrm{C}$ & 5.61584300 & 3.16943300 & -1.90993800 \\
\hline $\mathrm{C}$ & 1.95048100 & 0.26401700 & 8.52488400 \\
\hline $\mathrm{C}$ & 0.83405200 & 2.84588300 & 8.23269800 \\
\hline $\mathrm{C}$ & 2.39052700 & 1.33376300 & 9.31781100 \\
\hline $\mathrm{C}$ & 0.94094300 & 0.51442800 & 7.57857200 \\
\hline $\mathrm{C}$ & 0.39614300 & 1.78856100 & 7.42588000 \\
\hline $\mathrm{C}$ & 1.83151700 & 2.60658200 & 9.18295200 \\
\hline $\mathrm{C}$ & 0.26919700 & 4.23875300 & 8.05965200 \\
\hline $\mathrm{C}$ & -3.97064800 & 4.18558200 & 8.59788400 \\
\hline $\mathrm{C}$ & -1.74793400 & 3.92407500 & 9.47141100 \\
\hline $\mathrm{C}$ & -2.04411900 & 4.51535600 & 7.19843800 \\
\hline $\mathrm{C}$ & -3.43174600 & 4.49575100 & 7.34184000 \\
\hline $\mathrm{C}$ & -3.11604900 & 3.89847200 & 9.66444600 \\
\hline $\mathrm{C}$ & -4.29860800 & 4.79695000 & 6.20264200 \\
\hline $\mathrm{N}$ & -3.77509500 & 5.04337400 & 5.04746800 \\
\hline $\mathrm{N}$ & -4.66216600 & 5.33719300 & 4.01397200 \\
\hline $\mathrm{C}$ & -4.17653500 & 5.60949100 & 2.77556200 \\
\hline $\mathrm{C}$ & -4.51518200 & 4.51980500 & -8.41452800 \\
\hline $\mathrm{C}$ & -5.15208000 & 6.89599800 & -7.02204300 \\
\hline $\mathrm{C}$ & -5.85481100 & 4.91580600 & -8.23058800 \\
\hline $\mathrm{C}$ & -3.50768000 & 5.32961600 & -7.88004700 \\
\hline
\end{tabular}

Cage C1-3

$\begin{array}{cccc}\text { E }-4809.659543 \text { a.u. (zero imaginary freq.) } \\ \text { C } & 1.64666600 & -2.24218800 & 8.16810400 \\ \text { C } & -0.12934800 & -4.32771500 & 7.45597400 \\ \text { C } & 0.38444300 & -2.36104600 & 8.78091100 \\ \text { C } & 1.99535900 & -3.18640900 & 7.19906500 \\ \text { C } & 1.11717600 & -4.21812100 & 6.84111700 \\ \text { C } & -0.48878000 & -3.38614100 & 8.43349600 \\ \text { C } & -1.15009800 & -5.40382200 & 7.11673200 \\ \text { C } & 0.48848300 & -8.32899600 & 4.49046000\end{array}$




\begin{tabular}{|c|c|c|c|}
\hline $\mathrm{C}$ & -3.82202000 & 6.50542300 & -7.18864500 \\
\hline $\mathrm{C}$ & -6.17034100 & 6.08573100 & -7.54734800 \\
\hline $\mathrm{C}$ & -5.48600900 & 8.19475400 & -6.32190000 \\
\hline $\mathrm{C}$ & -7.40421600 & 7.41629600 & -2.58019300 \\
\hline $\mathrm{C}$ & -7.51306100 & 8.10242400 & -4.87739400 \\
\hline $\mathrm{C}$ & -5.41979400 & 7.52160300 & -3.93670400 \\
\hline $\mathrm{C}$ & -6.01154400 & 7.25727100 & -2.70670000 \\
\hline $\mathrm{C}$ & -8.15029000 & 7.83855200 & -3.67380400 \\
\hline $\mathrm{C}$ & -5.16528600 & 6.84130800 & -1.58951500 \\
\hline $\mathrm{N}$ & -5.70207500 & 6.58857600 & -0.44173700 \\
\hline $\mathrm{N}$ & -4.82548700 & 6.22247800 & 0.57839400 \\
\hline $\mathrm{C}$ & -5.31475700 & 5.94214200 & 1.81350800 \\
\hline $\mathrm{N}$ & -0.59834500 & -6.45271900 & 6.19073700 \\
\hline $\mathrm{N}$ & -5.94180000 & -2.60572200 & -6.86455800 \\
\hline $\mathrm{O}$ & -1.85466000 & -6.36075200 & -1.93805700 \\
\hline $\mathrm{O}$ & -2.84505600 & -4.29736000 & 0.77080900 \\
\hline $\mathrm{N}$ & -1.23769700 & 4.23957500 & 8.24547700 \\
\hline $\mathrm{N}$ & -6.16880600 & 7.94155000 & -4.98733400 \\
\hline $\mathrm{N}$ & 2.31009900 & 2.91652500 & -8.23775400 \\
\hline $\mathrm{N}$ & 7.90914900 & 0.25833900 & 4.95136300 \\
\hline $\mathrm{O}$ & 5.95740000 & 4.36341100 & -1.90364500 \\
\hline $\mathrm{O}$ & 5.57451700 & 1.02806500 & -0.75 \\
\hline $\mathrm{O}$ & -6.50579800 & 5.94665600 & 2.16503500 \\
\hline $\mathrm{O}$ & -2.98547800 & 5.60827700 & 5500 \\
\hline $\mathrm{H}$ & 0.08210500 & -1.64249600 & 9.53337900 \\
\hline $\mathrm{H}$ & 2.95741100 & -3.12514400 & 6.70955600 \\
\hline $\mathrm{H}$ & 1.42569400 & -4.926 & 0700 \\
\hline $\mathrm{H}$ & -1.45507400 & -3.4 & 8300 \\
\hline $\mathrm{H}$ & -2.01977800 & 7100 & 72300 \\
\hline $\mathrm{H}$ & -1.48232500 & 06800 & 10500 \\
\hline $\mathrm{H}$ & 0.90949000 & -9.06855800 & 0000 \\
\hline $\mathrm{H}$ & 0.27689200 & -7.48950600 & 7.77971400 \\
\hline $\mathrm{H}$ & -1.40293100 & -5.50458300 & 4.51740400 \\
\hline $\mathrm{H}$ & 1.28443300 & -9.22373600 & 6.29050000 \\
\hline $\mathrm{H}$ & -0.07973600 & -7.92719600 & 28200 \\
\hline $\mathrm{H}$ & -1.08735000 & -6.90244700 & 32700 \\
\hline $\mathrm{H}$ & -4.88186000 & 0.98502400 & -10.6 \\
\hline $\mathrm{H}$ & -5.18811700 & 2.72798400 & 10200 \\
\hline $\mathrm{H}$ & -6.30326700 & 0.68907300 & -5.92462000 \\
\hline $\mathrm{H}$ & -6.02586900 & -1.02885300 & -9.85268000 \\
\hline $\mathrm{H}$ & -7.52473500 & -2.03430300 & -8.12908000 \\
\hline $\mathrm{H}$ & 62200 & -1.34593300 & 200 \\
\hline $\mathrm{H}$ & -3.36744000 & -5.19984100 & -5.69264900 \\
\hline $\mathrm{H}$ & -5.73785500 & -3.37281800 & -8.79278400 \\
\hline $\mathrm{H}$ & -6.05445000 & -1.93343700 & -4.89290300 \\
\hline $\mathrm{H}$ & -4.05128300 & -5.07968300 & -8.10245700 \\
\hline $\mathrm{H}$ & -4.77693700 & -2.85233200 & -3.04561700 \\
\hline $\mathrm{H}$ & -3.64702900 & -3.78027000 & -1.25475700 \\
\hline $\mathrm{H}$ & -4.2 & 4.58 & 68100 \\
\hline $\mathrm{H}$ & -5.79607700 & 3.71293200 & -10.72010200 \\
\hline $\mathrm{H}$ & -4.41228500 & 2.86444800 & -11.44589400 \\
\hline $\mathrm{H}$ & 3.71646000 & -0.77649400 & 10.52342200 \\
\hline $\mathrm{H}$ & 3.30194800 & -2.47662600 & 10.22647000 \\
\hline $\mathrm{H}$ & 2.04378100 & -1.33676500 & 10.73791300 \\
\hline $\mathrm{H}$ & & -2.0 & \\
\hline $\mathrm{H}$ & 2.919 & -0.20 & 700 \\
\hline $\mathrm{H}$ & 4.90 & -0.23 & 1600 \\
\hline $\mathrm{H}$ & 7.18620600 & -2.02264600 & 7.80115500 \\
\hline $\mathrm{H}$ & 7.19317600 & -1.59179800 & 4.25175100 \\
\hline $\mathrm{H}$ & 8.23641000 & -1.69197500 & 5.69486500 \\
\hline $\mathrm{H}$ & 8.99381700 & 3.89293700 & 4.35882300 \\
\hline $\mathrm{H}$ & & 0.3 & 200 \\
\hline $\mathrm{H}$ & 7.04228200 & 18000 & 3.05537700 \\
\hline $\mathrm{H}$ & 9.57526600 & 2.72287800 & 6.49430600 \\
\hline $\mathrm{H}$ & 7.89165400 & 3.87797000 & 2.17035700 \\
\hline $\mathrm{H}$ & 6.84002900 & 3.85597700 & 0.10183600 \\
\hline $\mathrm{H}$ & -2.26147200 & 3.88480100 & -11.19313500 \\
\hline $\mathrm{H}$ & -2.73186600 & 1.97695400 & -7.36787000 \\
\hline $\mathrm{H}$ & 8600 & 1.47865200 & -7.32370500 \\
\hline $\mathrm{H}$ & 0.13571000 & 3.37266900 & -11.15295500 \\
\hline $\mathrm{H}$ & 2.00256300 & 2.23162300 & -10.21032600 \\
\hline $\mathrm{H}$ & 1.72626400 & 1.01828600 & -8.93360600 \\
\hline $\mathrm{H}$ & 4.15850900 & 5.12745600 & -5.70272000 \\
\hline $\mathrm{H}$ & 2.09273800 & 4.59968000 & -9.45028800 \\
\hline $\mathrm{H}$ & 2.58905600 & 1.31389600 & -6.93320700 \\
\hline $\mathrm{H}$ & 3.29747100 & 6.09460200 & -7.85014900 \\
\hline $\mathrm{H}$ & 3.68849300 & 1.44930600 & -4.80070300 \\
\hline $\mathrm{H}$ & 4.72397300 & 1.52102100 & -2.75860500 \\
\hline $\mathrm{H}$ & 3.17473000 & 1.18684600 & 10.04881800 \\
\hline $\mathrm{H}$ & 0.57984400 & -0.29337300 & 6.95560800 \\
\hline
\end{tabular}

$\begin{array}{cccc}\mathrm{H} & -0.37501400 & 1.95827600 & 6.68171100 \\ \mathrm{H} & 2.18001500 & 3.41721500 & 9.81345000 \\ \mathrm{H} & 0.45108900 & 4.62755300 & 7.05644600 \\ \mathrm{H} & 0.68117100 & 4.92902900 & 8.79723600 \\ \mathrm{H} & -5.04467700 & 4.16892500 & 8.73589300 \\ \mathrm{H} & -1.03796000 & 3.70580800 & 10.25552100 \\ \mathrm{H} & -1.57885300 & 4.75707700 & 6.25443200 \\ \mathrm{H} & -3.50654200 & 3.65573400 & 10.64219300 \\ \mathrm{H} & -5.37042300 & 4.80056000 & 6.39549800 \\ \mathrm{H} & -5.67750000 & 5.37156900 & 4.16538500 \\ \mathrm{H} & -6.65515700 & 4.30651000 & -8.63306700 \\ \mathrm{H} & -2.47030200 & 5.05320900 & -8.00929600 \\ \mathrm{H} & -3.02520800 & 7.12507200 & -6.79202300 \\ \mathrm{H} & -7.20871200 & 6.37623500 & -7.42680600 \\ \mathrm{H} & -6.18170600 & 8.80051300 & -6.90391100 \\ \mathrm{H} & -4.58609700 & 8.77350400 & -6.10984700 \\ \mathrm{H} & -7.87651700 & 7.21309500 & -1.62854800 \\ \mathrm{H} & -8.04107200 & 8.44659700 & -5.75412600 \\ \mathrm{H} & -4.35680600 & 7.41078100 & -4.10215600 \\ \mathrm{H} & -9.22000600 & 7.97380100 & -3.60477700 \\ \mathrm{H} & -4.09535400 & 6.77231000 & -1.78227400 \\ \mathrm{H} & -3.81011000 & 6.17842600 & 0.42957100\end{array}$

Cage C2-1

E -4809.673323 a.u. (zero imaginary freq.)

\begin{tabular}{|c|c|c|c|}
\hline $\mathrm{C}$ & -15.94234700 & 0.24585700 & -9.77445700 \\
\hline $\mathrm{C}$ & -17.25725300 & -2.00966400 & -8.68779000 \\
\hline $\mathrm{C}$ & -16.14063900 & -0.91323200 & -10.54152900 \\
\hline $\mathrm{C}$ & -16.40400100 & 0.24804100 & -8.44886600 \\
\hline $\mathrm{C}$ & -17.05774200 & -0.86183400 & -7.91248600 \\
\hline $\mathrm{C}$ & -16.78812900 & -2.02782100 & -10.00644400 \\
\hline $\mathrm{C}$ & -17.97891100 & -3.21263800 & -8.11745200 \\
\hline $\mathrm{C}$ & -15.71290000 & -4.82365200 & -4.85524900 \\
\hline $\mathrm{C}$ & -17.66258300 & -3.67930600 & -5.68527600 \\
\hline $\mathrm{C}$ & -16.01493500 & -4.42773500 & -7.21235300 \\
\hline $\mathrm{C}$ & -15.26088300 & -4.94754600 & -6.18075300 \\
\hline $\mathrm{C}$ & -16.93890600 & -4.17658200 & -4.62231300 \\
\hline $\mathrm{C}$ & -14.90080200 & -5.35205300 & -3.76481600 \\
\hline $\mathrm{N}$ & -15.28428900 & -5.18197400 & -2.53983200 \\
\hline $\mathrm{N}$ & -14.44345400 & -5.68900700 & -1.55986200 \\
\hline $\mathrm{C}$ & -14.76319800 & -5.52523700 & -0.24652700 \\
\hline $\mathrm{C}$ & -7.81952200 & -3.93236600 & 9.86351400 \\
\hline $\mathrm{C}$ & -9.13907700 & -6.24855400 & 8.91811300 \\
\hline $\mathrm{C}$ & -8.70446200 & -4.64635100 & 10.68726600 \\
\hline $\mathrm{C}$ & -7.62136400 & -4.39234800 & 8.55230900 \\
\hline $\mathrm{C}$ & -8.26649800 & -5.53834400 & 8.08596900 \\
\hline $\mathrm{C}$ & -9.35759900 & -5.78860900 & 10.22183100 \\
\hline $\mathrm{C}$ & -9.82525500 & -7.50550700 & 8.42624200 \\
\hline $\mathrm{C}$ & -12.50618600 & -6.51721200 & 5.23189400 \\
\hline $\mathrm{C}$ & -10.45657200 & -7.51344000 & 6.01096900 \\
\hline $\mathrm{C}$ & -11.94477100 & -6.53990100 & 7.57444900 \\
\hline $\mathrm{C}$ & -12.82236400 & -6.20171000 & 6.56484900 \\
\hline $\mathrm{C}$ & -11.29904900 & -7.18852100 & 4.96949500 \\
\hline $\mathrm{C}$ & -13.41831500 & -6.13036000 & 4.16017000 \\
\hline $\mathrm{N}$ & -13.09567000 & -6.37948100 & 2.93129400 \\
\hline $\mathrm{N}$ & -13.98724700 & -5.95024800 & 1.95974400 \\
\hline $\mathrm{C}$ & -13.68361600 & -6.13466000 & 0.64517500 \\
\hline $\mathrm{C}$ & -15.19509500 & 1.45818600 & -10.38217300 \\
\hline $\mathrm{C}$ & -15.74187700 & 1.72739500 & -11.82055900 \\
\hline $\mathrm{C}$ & -7.12919000 & -2.65215000 & 10.39561800 \\
\hline $\mathrm{C}$ & -6.57051600 & -2.92856100 & 11.82821000 \\
\hline $\mathrm{C}$ & -5.95248500 & -2.26014000 & 9.46900000 \\
\hline $\mathrm{C}$ & -3.78188300 & -1.60062800 & 7.77809300 \\
\hline $\mathrm{C}$ & -4.79028400 & -3.04897900 & 9.44288300 \\
\hline $\mathrm{C}$ & -6.00955700 & -1.14228900 & 8.62321300 \\
\hline $\mathrm{C}$ & -4.93767100 & -0.81247100 & 7.79247100 \\
\hline $\mathrm{C}$ & -3.71995600 & -2.72672400 & 8.60774800 \\
\hline $\mathrm{C}$ & -2.61013600 & -1.23662400 & 6.89056900 \\
\hline $\mathrm{C}$ & -3.76996200 & -1.71355400 & 2.79255400 \\
\hline $\mathrm{C}$ & -3.23271300 & -0.29550100 & 4.66289900 \\
\hline $\mathrm{C}$ & -3.10328600 & -2.64667900 & 4.91070700 \\
\hline $\mathrm{C}$ & -3.49002900 & -2.83009100 & 3.59916800 \\
\hline $\mathrm{C}$ & -3.62983700 & -0.43051400 & 3.35002800 \\
\hline $\mathrm{C}$ & -4.20881200 & -1.91029400 & 1.41539900 \\
\hline $\mathrm{N}$ & -4.51818600 & -0.88278800 & 0.69081000 \\
\hline $\mathrm{N}$ & -4.94583300 & -1.15167900 & -0.60144700 \\
\hline $\mathrm{C}$ & -5.32082500 & -0.12539800 & -1.41341000 \\
\hline $\mathrm{C}$ & -13.67966300 & 1.15168800 & -10.46615700 \\
\hline $\mathrm{C}$ & -10.90057100 & 0.64946600 & -10.61837200 \\
\hline $\mathrm{C}$ & -12.84017800 & 1.96663100 & -11.24257800 \\
\hline
\end{tabular}




\begin{tabular}{|c|c|c|c|}
\hline $\mathrm{C}$ & -13.09748200 & 0.08961700 & -9.75703400 \\
\hline $\mathrm{C}$ & -11.72763000 & -0.16354400 & -9.83539500 \\
\hline $\mathrm{C}$ & -11.46827600 & 1.72119300 & -11.31707100 \\
\hline $\mathrm{C}$ & -9.41560600 & 0.37123800 & -10.72298100 \\
\hline $\mathrm{C}$ & -7.62005300 & 0.90269500 & -6.86745400 \\
\hline $\mathrm{C}$ & -8.30461300 & -0.53308300 & -8.67568200 \\
\hline $\mathrm{C}$ & -8.63070000 & 1.80514700 & -8.85963200 \\
\hline $\mathrm{C}$ & -8.07019200 & 2.00511200 & -7.61497700 \\
\hline $\mathrm{C}$ & -7.74456500 & -0.38199600 & -7.42514400 \\
\hline $\mathrm{C}$ & -7.05543000 & 1.11353900 & -5.53877800 \\
\hline $\mathrm{N}$ & -6.68183000 & 0.09262400 & -4.83548000 \\
\hline $\mathrm{N}$ & -6.17932100 & 0.36819000 & -3.57251700 \\
\hline $\mathrm{C}$ & -5.78594900 & -0.65681200 & -2.76738000 \\
\hline $\mathrm{C}$ & -8.15836000 & -1.49679500 & 10.46086000 \\
\hline $\mathrm{C}$ & -9.99263300 & 0.65501500 & 10.55033400 \\
\hline $\mathrm{C}$ & -7.88788100 & -0.34885400 & 11.22277600 \\
\hline $\mathrm{C}$ & -9.36096300 & -1.54096400 & 9.73808600 \\
\hline $\mathrm{C}$ & -10.27003900 & -0.48412700 & 9.78595700 \\
\hline $\mathrm{C}$ & -8.79149600 & 0.71454600 & 11.26596000 \\
\hline $\mathrm{C}$ & -10.97813100 & 1.80350800 & 10.61600700 \\
\hline $\mathrm{C}$ & -11.40695000 & 3.53218200 & 6.71760700 \\
\hline $\mathrm{C}$ & -12.30712700 & 2.26225200 & 8.55485100 \\
\hline $\mathrm{C}$ & -10.12008800 & 3.15490200 & 8.71885000 \\
\hline $\mathrm{C}$ & -10.22710900 & 3.71057200 & 7.46012200 \\
\hline $\mathrm{C}$ & -12.45641400 & 2.79421600 & 7.29267800 \\
\hline $\mathrm{C}$ & -11.51875300 & 4.09623800 & 5.37572100 \\
\hline $\mathrm{N}$ & -12.59910300 & 3.88076500 & 4.69575500 \\
\hline $\mathrm{N}$ & -12.65182900 & 4.41556100 & 3.41848800 \\
\hline $\mathrm{C}$ & -13.75160200 & 4.18452200 & 2.64888200 \\
\hline $\mathrm{C}$ & -15.43109700 & 2.71269100 & -9.50620400 \\
\hline $\mathrm{C}$ & -15.92161100 & 4.97321700 & -7.87770400 \\
\hline $\mathrm{C}$ & -16.67448700 & 3.36481100 & -9.53134600 \\
\hline $\mathrm{C}$ & -14.44295200 & 3.20909200 & -8.64210400 \\
\hline $\mathrm{C}$ & -14.68012600 & 4.32810600 & -7.84313700 \\
\hline $\mathrm{C}$ & -16.91880500 & 4.47816500 & -8.72589500 \\
\hline $\mathrm{C}$ & -16.18447000 & 6.19823600 & -7.02592100 \\
\hline $\mathrm{C}$ & -15.87021700 & 5.10938500 & -2.89263200 \\
\hline $\mathrm{C}$ & -14.99744800 & 6.24201500 & -4.83016600 \\
\hline $\mathrm{C}$ & -17.07011100 & 5.10776500 & -4.98170100 \\
\hline $\mathrm{C}$ & -16.98695300 & 4.72981700 & -3.65732500 \\
\hline $\mathrm{C}$ & -14.86791600 & 5.88100000 & -3.50659800 \\
\hline $\mathrm{C}$ & -15.77042900 & 4.68915700 & -1.49934600 \\
\hline $\mathrm{N}$ & -14.71852400 & 5.00589800 & -0.81364100 \\
\hline $\mathrm{N}$ & -14.68163600 & 4.55450000 & 0.49675200 \\
\hline $\mathrm{C}$ & -13.58970700 & 4.81365200 & 1.26815500 \\
\hline $\mathrm{N}$ & -17.20002700 & -3.80740300 & -6.96023400 \\
\hline $\mathrm{O}$ & -15.76840200 & -4.96127100 & 0.21075800 \\
\hline $\mathrm{O}$ & -12.67953100 & -6.69953900 & 0.18650900 \\
\hline $\mathrm{N}$ & -10.78188800 & -7.18838900 & 7.29333400 \\
\hline $\mathrm{N}$ & -11.15126500 & 2.44493100 & 9.25237000 \\
\hline $\mathrm{N}$ & -16.08684200 & 5.85605700 & -5.55189600 \\
\hline $\mathrm{N}$ & -8.74026700 & 0.55135700 & -9.37651300 \\
\hline $\mathrm{N}$ & -2.97793400 & -1.39422400 & 5.42738800 \\
\hline $\mathrm{O}$ & -5.79460400 & -1.86578200 & -3.04329100 \\
\hline $\mathrm{O}$ & -5.31380300 & 1.08358000 & -1.13734300 \\
\hline $\mathrm{O}$ & -12.58075800 & 5.45467100 & 0.93885100 \\
\hline $\mathrm{O}$ & -14.76304800 & 3.54550000 & 2.97527900 \\
\hline $\mathrm{H}$ & -15.78052800 & -0.95843500 & -11.56139300 \\
\hline $\mathrm{H}$ & -16.25689100 & 1.12455600 & -7.83285900 \\
\hline $\mathrm{H}$ & -17.41068800 & -0.83288600 & -6.88696400 \\
\hline $\mathrm{H}$ & -16.93252500 & -2.91268400 & -10.61673300 \\
\hline $\mathrm{H}$ & -18.08931400 & -3.99931800 & -8.86554400 \\
\hline $\mathrm{H}$ & -18.96023800 & -2.94813600 & -7.72097000 \\
\hline $\mathrm{H}$ & -18.61118100 & -3.17984900 & -5.55459300 \\
\hline $\mathrm{H}$ & -15.70401800 & -4.48865700 & -8.24536700 \\
\hline $\mathrm{H}$ & -14.32533000 & -5.44210000 & -6.40431500 \\
\hline $\mathrm{H}$ & -17.31925300 & -4.06563100 & -3.61761500 \\
\hline $\mathrm{H}$ & -13.97985600 & -5.86491500 & -4.03842000 \\
\hline $\mathrm{H}$ & -13.57029100 & -6.17639400 & -1.79668100 \\
\hline $\mathrm{H}$ & -8.89890500 & -4.30951000 & 11.69771900 \\
\hline $\mathrm{H}$ & -6.95376600 & -3.85542200 & 7.89256600 \\
\hline $\mathrm{H}$ & -8.09131100 & -5.87738700 & 7.07023000 \\
\hline $\mathrm{H}$ & -10.03508100 & -6.32657300 & 10.87590300 \\
\hline $\mathrm{H}$ & -10.41544500 & -7.96961700 & 9.21783200 \\
\hline $\mathrm{H}$ & -9.11039300 & -8.22924000 & 8.03183700 \\
\hline $\mathrm{H}$ & -9.52317200 & -8.03499300 & 5.85862300 \\
\hline $\mathrm{H}$ & -12.13709200 & -6.30914300 & 8.61248400 \\
\hline $\mathrm{H}$ & -13.74287800 & -5.69034000 & 6.81159000 \\
\hline $\mathrm{H}$ & -11.02612200 & -7.45028800 & 3.95790700 \\
\hline $\mathrm{H}$ & -14.33795600 & -5.62328600 & 4.44770300 \\
\hline
\end{tabular}

\begin{tabular}{|c|c|c|c|}
\hline $\mathrm{H}$ & -14.85707100 & -5.45985300 & 2.20170200 \\
\hline $\mathrm{H}$ & -15.35316900 & 2.67325900 & -12.20619100 \\
\hline $\mathrm{H}$ & -16.83343800 & 1.77937300 & -11.80801100 \\
\hline $\mathrm{H}$ & -15.44040100 & 0.92528500 & -12.49880000 \\
\hline $\mathrm{H}$ & -5.94415100 & -2.09629400 & 12.15882100 \\
\hline $\mathrm{H}$ & -5.96772100 & -3.83996600 & 11.83088500 \\
\hline $\mathrm{H}$ & -7.39055600 & -3.05068200 & 12.54007500 \\
\hline $\mathrm{H}$ & -4.71750500 & -3.93066400 & 10.06749700 \\
\hline $\mathrm{H}$ & -6.89375300 & -0.51983000 & 8.61641700 \\
\hline $\mathrm{H}$ & -5.00450800 & 0.06300400 & 7.15488300 \\
\hline $\mathrm{H}$ & -2.83151600 & -3.34868700 & 8.60669400 \\
\hline $\mathrm{H}$ & -1.75568800 & -1.88961400 & 7.07404600 \\
\hline $\mathrm{H}$ & -2.30988800 & -0.19599400 & 7.02157400 \\
\hline $\mathrm{H}$ & -3.10568400 & 0.66900300 & 5.13198400 \\
\hline $\mathrm{H}$ & -2.89010700 & -3.47295300 & 5.57333900 \\
\hline $\mathrm{H}$ & -3.58032600 & -3.83415200 & 3.20759200 \\
\hline $\mathrm{H}$ & -3.83124100 & 0.45000000 & 2.75791900 \\
\hline $\mathrm{H}$ & -4.26967200 & -2.93635800 & 1.05641000 \\
\hline $\mathrm{H}$ & -5.00341500 & -2.11187200 & -0.96265300 \\
\hline $\mathrm{H}$ & -13.25014100 & 2.80771200 & -11.78733600 \\
\hline $\mathrm{H}$ & -13.71925800 & -0.54776400 & -9.14330500 \\
\hline $\mathrm{H}$ & -11.30290800 & -0.99508400 & -9.28294500 \\
\hline $\mathrm{H}$ & -10.83776700 & 2.36104600 & -11.92479800 \\
\hline $\mathrm{H}$ & -8.93389900 & 1.06178200 & -11.41655800 \\
\hline $\mathrm{H}$ & -9.21470300 & -0.65554100 & -11.03214200 \\
\hline $\mathrm{H}$ & -8.41746500 & -1.49926800 & -9.14474800 \\
\hline $\mathrm{H}$ & -9.00329900 & 2.61874200 & -9.46519600 \\
\hline $\mathrm{H}$ & -7.98959800 & 3.01045000 & -7.22474600 \\
\hline $\mathrm{H}$ & -7.40763500 & -1.25196700 & -6.88105000 \\
\hline $\mathrm{H}$ & -6.98268900 & 2.14175600 & -5.18816900 \\
\hline $\mathrm{H}$ & -6.11798100 & 1.32883000 & -3.21312900 \\
\hline $\mathrm{H}$ & -6.96404400 & -0.27025700 & 11.78165000 \\
\hline $\mathrm{H}$ & -9.59238900 & -2.41022300 & 9.13784000 \\
\hline $\mathrm{H}$ & -11.19455800 & -0.54550600 & 9.22144200 \\
\hline $\mathrm{H}$ & -8.56290100 & 1.59215700 & 11.86095700 \\
\hline $\mathrm{H}$ & -10.62946200 & 2.58413100 & 11.29363900 \\
\hline $\mathrm{H}$ & -11.97024300 & 1.47003600 & 10.92377200 \\
\hline $\mathrm{H}$ & -13.08880800 & 1.69457300 & 9.03733700 \\
\hline $\mathrm{H}$ & -9.23093400 & 3.25513500 & 9.32423300 \\
\hline $\mathrm{H}$ & -9.39700700 & 4.27496000 & 7.05767200 \\
\hline $\mathrm{H}$ & -13.37816200 & 2.64076900 & 6.75129400 \\
\hline $\mathrm{H}$ & -10.67304900 & 4.66912400 & 4.99936200 \\
\hline $\mathrm{H}$ & -11.87450300 & 4.96086500 & 3.02593200 \\
\hline $\mathrm{H}$ & -17.46785000 & 3.00102700 & -10.17208800 \\
\hline $\mathrm{H}$ & -13.47890700 & 2.72133500 & -8.59631000 \\
\hline $\mathrm{H}$ & -13.89555100 & 4.69532500 & -7.18993700 \\
\hline $\mathrm{H}$ & -17.88631800 & 4.96684700 & -8.76054600 \\
\hline $\mathrm{H}$ & -17.18910800 & 6.58875200 & -7.19576700 \\
\hline $\mathrm{H}$ & -15.45264200 & 6.98680500 & -7.20890600 \\
\hline $\mathrm{H}$ & -14.25553700 & 6.83768100 & -5.34128700 \\
\hline $\mathrm{H}$ & -17.90266500 & 4.83146800 & -5.61234600 \\
\hline $\mathrm{H}$ & -17.78304100 & 4.14085400 & -3.22268500 \\
\hline $\mathrm{H}$ & -13.99713400 & 6.19332600 & -2.94925400 \\
\hline $\mathrm{H}$ & -16.59667300 & 4.10695800 & -1.09454500 \\
\hline $\mathrm{H}$ & -15.45029300 & 4.00422900 & 0.89979200 \\
\hline
\end{tabular}

Cage C2-2

$\begin{array}{lccc}\text { E -4809.673278 a.u. (zero imaginary freq.) } \\ \text { C } & -5.58056200 & 1.00569300 & -9.77662900 \\ \text { C } & -6.88421300 & -1.28383800 & -8.74870300 \\ \text { C } & -5.75938900 & -0.13978000 & -10.56853800 \\ \text { C } & -6.05752800 & 0.97783600 & -8.45689800 \\ \text { C } & -6.70600300 & -0.14884300 & -7.94969100 \\ \text { C } & -6.40121400 & -1.27102300 & -10.06255600 \\ \text { C } & -7.59702800 & -2.50682400 & -8.20944300 \\ \text { C } & -5.33510100 & -4.15763800 & -4.96423100 \\ \text { C } & -7.28203800 & -3.00036000 & -5.78258300 \\ \text { C } & -5.63709900 & -3.73899300 & -7.31743100 \\ \text { C } & -4.88368100 & -4.26979400 & -6.29091800 \\ \text { C } & -6.55915600 & -3.50920900 & -4.72463300 \\ \text { C } & -4.52473800 & -4.69988700 & -3.87929800 \\ \text { N } & -4.91016500 & -4.54472700 & -2.65298100 \\ \text { N } & -4.07159900 & -5.06410600 & -1.67744300 \\ \text { C } & -4.39603200 & -4.91727500 & -0.36322800 \\ \text { C } & 2.48587000 & -3.45877800 & 9.82114100 \\ \text { C } & 1.17266700 & -5.75997100 & 8.83021300 \\ \text { C } & 1.58432800 & -4.17758000 & 10.62267500 \\ \text { C } & 2.70489900 & -3.90704500 & 8.50937500 \\ \text { C } & 2.06350600 & -5.04606900 & 8.02101300 \\ \text { C } & 0.93420800 & -5.31200900 & 10.13477100\end{array}$




\begin{tabular}{|c|c|c|c|}
\hline $\mathrm{C}$ & 0.48930700 & -7.00893100 & 8.31474900 \\
\hline $\mathrm{C}$ & -2.17055400 & -5.97852900 & 5.11507800 \\
\hline $\mathrm{C}$ & -0.11960400 & -6.97247600 & 5.89326800 \\
\hline $\mathrm{C}$ & -1.63052100 & -6.04305300 & 7.46186700 \\
\hline $\mathrm{C}$ & -2.50147800 & -5.69188800 & 6.45093900 \\
\hline $\mathrm{C}$ & -0.95526200 & -6.63426900 & 4.85070900 \\
\hline $\mathrm{C}$ & -3.07624200 & -5.57864100 & 4.04257900 \\
\hline $\mathrm{N}$ & -2.74186600 & -5.80484100 & 2.81234400 \\
\hline $\mathrm{N}$ & -3.62868800 & -5.36596200 & 1.84083800 \\
\hline $\mathrm{C}$ & -3.31719900 & -5.53216100 & 0.52559700 \\
\hline $\mathrm{C}$ & -4.83513400 & 2.23469700 & -10.35173100 \\
\hline $\mathrm{C}$ & -5.37229500 & 2.53267200 & -11.78815000 \\
\hline $\mathrm{C}$ & 3.17214900 & -2.18755000 & 10.37970400 \\
\hline $\mathrm{C}$ & 3.72209500 & -2.49074700 & 11.81027500 \\
\hline $\mathrm{C}$ & 4.35395100 & -1.77688700 & 9.46751600 \\
\hline $\mathrm{C}$ & 6.53252700 & -1.08302100 & 7.80090900 \\
\hline $\mathrm{C}$ & 5.51828400 & -2.56249500 & 9.43444600 \\
\hline $\mathrm{C}$ & 4.29939000 & -0.64409200 & 8.64167000 \\
\hline $\mathrm{C}$ & 5.37526200 & -0.29717600 & 7.82333000 \\
\hline $\mathrm{C}$ & 6.59239200 & -2.22345200 & 8.61084800 \\
\hline $\mathrm{C}$ & 7.70705200 & -0.70194200 & 6.92437700 \\
\hline $\mathrm{C}$ & 6.56588700 & -1.12217500 & 2.81478500 \\
\hline $\mathrm{C}$ & 7.09612700 & 0.26996500 & 4.70658300 \\
\hline $\mathrm{C}$ & 7.22190300 & -2.08453000 & 4.92311500 \\
\hline $\mathrm{C}$ & 6.84105400 & -2.24982900 & 3.60736100 \\
\hline $\mathrm{C}$ & 6.70485000 & 0.15296400 & 3.39029400 \\
\hline $\mathrm{C}$ & 6.13225800 & -1.29859600 & 1.43311100 \\
\hline $\mathrm{N}$ & 5.82563900 & -0.25965700 & 0.72391600 \\
\hline $\mathrm{N}$ & 5.40135900 & -0.50550000 & -0.57370300 \\
\hline $\mathrm{C}$ & 5.02920100 & 0.53621800 & -1.36739300 \\
\hline $\mathrm{C}$ & -3.31781400 & 1.93553700 & -10.42856700 \\
\hline $\mathrm{C}$ & -0.53663400 & 1.44009900 & -10.56814400 \\
\hline $\mathrm{C}$ & -2.47646900 & 2.75401000 & -11.19906500 \\
\hline $\mathrm{C}$ & -2.73610300 & 0.87440500 & -9.71726500 \\
\hline $\mathrm{C}$ & -1.36541300 & 0.62455000 & -9.78939500 \\
\hline $\mathrm{C}$ & -1.10353900 & 2.51175400 & -11.26736500 \\
\hline $\mathrm{C}$ & 0.94852800 & 1.16218600 & -10.67189400 \\
\hline $\mathrm{C}$ & 2.73941700 & 1.65113900 & -6.80884300 \\
\hline $\mathrm{C}$ & 2.05388100 & 0.23525400 & -8.63237900 \\
\hline $\mathrm{C}$ & 1.73362400 & 2.57603800 & -8.79327300 \\
\hline $\mathrm{C}$ & 2.29274600 & 2.76204200 & -7.54573400 \\
\hline $\mathrm{C}$ & 2.61230300 & 0.37245000 & -7.37960600 \\
\hline $\mathrm{C}$ & 3.30347700 & 1.84603000 & -5.47731200 \\
\hline $\mathrm{N}$ & 3.67593000 & 0.81554000 & -4.78763900 \\
\hline $\mathrm{N}$ & 4.17755200 & 1.07051300 & -3.52018400 \\
\hline $\mathrm{C}$ & 4.56890300 & 0.03076900 & -2.73279300 \\
\hline $\mathrm{C}$ & 2.14148700 & -1.03457500 & 10.45947700 \\
\hline $\mathrm{C}$ & 0.30572600 & 1.11506200 & 10.57214500 \\
\hline $\mathrm{C}$ & 2.40303900 & 0.09762100 & 11.24758000 \\
\hline $\mathrm{C}$ & 0.94709900 & -1.06389100 & 9.72238300 \\
\hline $\mathrm{C}$ & 0.03726500 & -0.00835000 & 9.78165000 \\
\hline $\mathrm{C}$ & 1.49878200 & 1.16008100 & 11.30207400 \\
\hline $\mathrm{C}$ & -0.68095100 & 2.26187600 & 10.65070700 \\
\hline $\mathrm{C}$ & -1.11155200 & 4.03131200 & 6.77124500 \\
\hline $\mathrm{C}$ & -2.01257600 & 2.74525900 & 8.59681900 \\
\hline $\mathrm{C}$ & 0.17773000 & 3.62915400 & 8.76603900 \\
\hline $\mathrm{C}$ & 0.07034200 & 4.19782000 & 7.51330300 \\
\hline $\mathrm{C}$ & -2.16233400 & 3.29047100 & 7.34020200 \\
\hline $\mathrm{C}$ & -1.22335200 & 4.61295100 & 5.43702100 \\
\hline $\mathrm{N}$ & -2.30454800 & 4.41085200 & 4.75444300 \\
\hline $\mathrm{N}$ & -2.35565800 & 4.96687000 & 3.48596600 \\
\hline $\mathrm{C}$ & -3.45578200 & 4.75094600 & 2.71266400 \\
\hline $\mathrm{C}$ & -5.08561500 & 3.47017900 & -9.45322500 \\
\hline $\mathrm{C}$ & -5.60109100 & 5.70367400 & -7.79568100 \\
\hline $\mathrm{C}$ & -6.33691400 & 4.10763000 & -9.46863900 \\
\hline $\mathrm{C}$ & -4.10286600 & 3.96625600 & -8.58307600 \\
\hline $\mathrm{C}$ & -4.35261400 & 5.07200000 & -7.76944800 \\
\hline $\mathrm{C}$ & -6.59351000 & 5.20769000 & -8.64907100 \\
\hline $\mathrm{C}$ & -5.87612900 & 6.91631500 & -6.93031700 \\
\hline $\mathrm{C}$ & -5.57456100 & 5.77480800 & -2.81072000 \\
\hline $\mathrm{C}$ & -4.69783900 & 6.93562200 & -4.72965600 \\
\hline $\mathrm{C}$ & -6.76826200 & 5.80034800 & -4.90298300 \\
\hline $\mathrm{C}$ & -6.68869800 & 5.40459000 & -3.58359600 \\
\hline $\mathrm{C}$ & -4.57200200 & 6.55711200 & -3.41061600 \\
\hline $\mathrm{C}$ & -5.47635000 & 5.33233700 & -1.42431900 \\
\hline $\mathrm{N}$ & -4.42400300 & 5.63624600 & -0.73350400 \\
\hline $\mathrm{N}$ & -4.38676800 & 5.16143100 & 0.56853700 \\
\hline $\mathrm{C}$ & -3.29496300 & 5.40727200 & 1.34437900 \\
\hline $\mathrm{N}$ & -6.82070900 & -3.11847800 & -7.05910000 \\
\hline $\mathrm{O}$ & -5.40514300 & -4.36276500 & 0.09706900 \\
\hline
\end{tabular}

\begin{tabular}{|c|c|c|c|}
\hline $\mathrm{O}$ & -2.30817700 & -6.08630300 & 0.06478000 \\
\hline $\mathrm{N}$ & -0.46000000 & -6.67662000 & 7.17898300 \\
\hline $\mathrm{N}$ & -0.85510800 & 2.91767800 & 9.29429900 \\
\hline $\mathrm{N}$ & -5.78382700 & 6.55675800 & -5.46027800 \\
\hline $\mathrm{N}$ & 1.62212600 & 1.32779900 & -9.32303600 \\
\hline $\mathrm{N}$ & 7.34610200 & -0.83933500 & 5.45723400 \\
\hline $\mathrm{O}$ & 4.56198800 & -1.17272700 & -3.03164300 \\
\hline $\mathrm{O}$ & 5.03514200 & 1.73962700 & -1.06806900 \\
\hline $\mathrm{O}$ & -2.28693800 & 6.05564300 & 1.02691500 \\
\hline $\mathrm{O}$ & -4.46729200 & 4.10621400 & 3.02749800 \\
\hline $\mathrm{H}$ & -5.38762000 & -0.16138700 & -11.58498200 \\
\hline $\mathrm{H}$ & -5.92603000 & 1.84369800 & -7.82254100 \\
\hline $\mathrm{H}$ & -7.07079700 & -0.14322000 & -6.92796400 \\
\hline $\mathrm{H}$ & -6.52955600 & -2.14492300 & -10.69180000 \\
\hline $\mathrm{H}$ & -7.69225600 & -3.27817100 & -8.97539300 \\
\hline $\mathrm{H}$ & -8.58453400 & -2.26208600 & -7.81547400 \\
\hline $\mathrm{H}$ & -8.22943500 & -2.49994000 & -5.64703600 \\
\hline $\mathrm{H}$ & -5.32679200 & -3.79113800 & -8.35110500 \\
\hline $\mathrm{H}$ & -3.94940500 & -4.76450100 & -6.51943400 \\
\hline $\mathrm{H}$ & -6.93871100 & -3.40660100 & -3.71874800 \\
\hline $\mathrm{H}$ & -3.60405500 & -5.21033800 & -4.15821100 \\
\hline $\mathrm{H}$ & -3.19696900 & -5.54690700 & -1.91814800 \\
\hline $\mathrm{H}$ & 1.37451600 & -3.85031700 & 11.63323700 \\
\hline $\mathrm{H}$ & 3.38593600 & -3.36707200 & 7.86620500 \\
\hline $\mathrm{H}$ & 2.25576000 & -5.37650900 & 7.00555500 \\
\hline $\mathrm{H}$ & 0.24373400 & -5.85366600 & 10.77199900 \\
\hline $\mathrm{H}$ & -0.10728800 & -7.48383300 & 9.09502700 \\
\hline $\mathrm{H}$ & 1.20659000 & -7.72774900 & 7.91570600 \\
\hline $\mathrm{H}$ & 0.81987300 & -7.48247900 & 5.73925800 \\
\hline $\mathrm{H}$ & -1.83465900 & -5.83468300 & 8.50235600 \\
\hline $\mathrm{H}$ & -3.42834200 & -5.19266800 & 6.69880200 \\
\hline $\mathrm{H}$ & -0.67079400 & -6.87336200 & 3.83669000 \\
\hline $\mathrm{H}$ & -4.00160000 & -5.08269900 & 4.33126200 \\
\hline $\mathrm{H}$ & -4.50263400 & -4.88344800 & 2.08394500 \\
\hline $\mathrm{H}$ & -4.98604700 & 3.48891600 & -12.14973700 \\
\hline $\mathrm{H}$ & -6.46409000 & 2.57907200 & -11.78221400 \\
\hline $\mathrm{H}$ & -5.06181200 & 1.74724800 & -12.48172900 \\
\hline $\mathrm{H}$ & 4.34480400 & -1.66430300 & 12.16173000 \\
\hline $\mathrm{H}$ & 4.32635600 & -3.40106200 & 11.79888400 \\
\hline $\mathrm{H}$ & 2.89724200 & -2.62857400 & 12.51365200 \\
\hline $\mathrm{H}$ & 5.58958700 & -3.45475900 & 10.04405000 \\
\hline $\mathrm{H}$ & 3.41397000 & -0.02335400 & 8.64113300 \\
\hline $\mathrm{H}$ & 5.31067600 & 0.58954900 & 7.20132000 \\
\hline $\mathrm{H}$ & 7.48207900 & -2.84357500 & 8.60354500 \\
\hline $\mathrm{H}$ & 8.56295400 & -1.35469400 & 7.10184400 \\
\hline $\mathrm{H}$ & 8.00314700 & 0.33764200 & 7.07175500 \\
\hline $\mathrm{H}$ & 7.22185600 & 1.22797800 & 5.18915200 \\
\hline $\mathrm{H}$ & 7.43159700 & -2.91982900 & 5.57550700 \\
\hline $\mathrm{H}$ & 6.75159600 & -3.24847800 & 3.20196200 \\
\hline $\mathrm{H}$ & 6.50700000 & 1.04147000 & 2.80903800 \\
\hline $\mathrm{H}$ & 6.07262500 & -2.31904400 & 1.05859400 \\
\hline $\mathrm{H}$ & 5.34451900 & -1.45879500 & -0.95280000 \\
\hline $\mathrm{H}$ & -2.88554400 & 3.59461700 & -11.74507300 \\
\hline $\mathrm{H}$ & -3.35928900 & 0.23448700 & -9.10754600 \\
\hline $\mathrm{H}$ & -0.94155900 & -0.20704900 & -9.23638000 \\
\hline $\mathrm{H}$ & -0.47178200 & 3.15377400 & -11.87150200 \\
\hline $\mathrm{H}$ & 1.43176900 & 1.85912400 & -11.35797100 \\
\hline $\mathrm{H}$ & 1.14918900 & 0.13838500 & -10.99106900 \\
\hline $\mathrm{H}$ & 1.93925700 & -0.72597500 & -9.11109700 \\
\hline $\mathrm{H}$ & 1.36385900 & 3.39659400 & -9.39116900 \\
\hline $\mathrm{H}$ & 2.37509400 & 3.76328000 & -7.14540500 \\
\hline $\mathrm{H}$ & 2.94604500 & -0.50362700 & -6.84342000 \\
\hline $\mathrm{H}$ & 3.37674700 & 2.86973300 & -5.11394800 \\
\hline $\mathrm{H}$ & 4.23710200 & 2.02452200 & -3.14343300 \\
\hline $\mathrm{H}$ & 3.32084300 & 0.16532800 & 11.81765700 \\
\hline $\mathrm{H}$ & 0.72273500 & -1.92043300 & 9.10146100 \\
\hline $\mathrm{H}$ & -0.88051400 & -0.05830400 & 9.20522000 \\
\hline $\mathrm{H}$ & 1.72091000 & 2.02562600 & 11.91684500 \\
\hline $\mathrm{H}$ & -0.33271900 & 3.03509700 & 11.33703900 \\
\hline $\mathrm{H}$ & -1.67269700 & 1.92411700 & 10.95498400 \\
\hline $\mathrm{H}$ & -2.79495900 & 2.17508100 & 9.07522700 \\
\hline $\mathrm{H}$ & 1.06837400 & 3.71997000 & 9.37073800 \\
\hline $\mathrm{H}$ & 0.90148300 & 4.76363900 & 7.11505100 \\
\hline $\mathrm{H}$ & -3.08558400 & 3.14576700 & 6.79897500 \\
\hline $\mathrm{H}$ & -0.37617900 & 5.18864300 & 5.06834400 \\
\hline $\mathrm{H}$ & -1.57787500 & 5.51867300 & 3.10351500 \\
\hline $\mathrm{H}$ & -7.12646900 & 3.74249000 & -10.11343000 \\
\hline $\mathrm{H}$ & -3.13289200 & 3.48978300 & -8.54473400 \\
\hline $\mathrm{H}$ & -3.57158700 & 5.44029600 & -7.11261000 \\
\hline $\mathrm{H}$ & -7.56660500 & 5.68561300 & -8.67697500 \\
\hline
\end{tabular}




$\begin{array}{lrrr}\mathrm{H} & -6.88223200 & 7.30270500 & -7.10064000 \\ \mathrm{H} & -5.14812600 & 7.71142200 & -7.09979400 \\ \mathrm{H} & -3.95531700 & 7.53908100 & -5.23057500 \\ \mathrm{H} & -7.59829400 & 5.53123000 & -5.53992600 \\ \mathrm{H} & -7.48504700 & 4.80840500 & -3.15938500 \\ \mathrm{H} & -3.70360900 & 6.86311600 & -2.84605400 \\ \mathrm{H} & -6.30319800 & 4.74423200 & -1.02936700 \\ \mathrm{H} & -5.15473400 & 4.60276200 & 0.96131900\end{array}$

Cage C2-3

E -4809.673262 a.u. (zero imaginary freq.)

\begin{tabular}{|c|c|c|c|}
\hline $\mathrm{C}$ & -15.79263300 & 0.55849500 & -9.89756700 \\
\hline$C$ & -17.07115400 & -1.73150600 & -8.83952700 \\
\hline $\mathrm{C}$ & -15.95486900 & -0.60081700 & -10.67287800 \\
\hline$C$ & -16.27147500 & 0.54341900 & -8.57821700 \\
\hline $\mathrm{C}$ & -16.90753700 & -0.58353700 & -8.05611100 \\
\hline $\mathrm{C}$ & -16.58444300 & -1.73215000 & -10.15199500 \\
\hline $\mathrm{C}$ & -17.77416300 & -2.95380100 & -8.28624800 \\
\hline $\mathrm{C}$ & -15.52828900 & -4.53057100 & -4.99376300 \\
\hline $\mathrm{C}$ & -17.47363400 & -3.39658700 & -5.84800100 \\
\hline $\mathrm{C}$ & -15.81449900 & -4.15724600 & $-7.3565420 c$ \\
\hline $\mathrm{C}$ & -15.06684900 & -4.66639400 & $-6.3148130 c$ \\
\hline $\mathrm{C}$ & -16.75673400 & -3.88287700 & $-4.7755530 \mathrm{C}$ \\
\hline $\mathrm{C}$ & -14.72438600 & -5.04734000 & $-3.8915460 c$ \\
\hline $\mathrm{N}$ & -15.11854100 & -4.86424300 & -2.67182200 \\
\hline $\mathrm{N}$ & -14.28668800 & -5.36062700 & -1.6791480 \\
\hline $\mathrm{C}$ & -14.61706600 & -5.18193400 & -0.37033400 \\
\hline $\mathrm{C}$ & -7.80324800 & -3.49633500 & 9.82633400 \\
\hline $\mathrm{C}$ & -9.08381300 & -5.82480000 & 8.85767000 \\
\hline $\mathrm{C}$ & -8.65273100 & -4.24955800 & 10.65183800 \\
\hline $\mathrm{C}$ & -7.62044900 & -3.92379300 & 8.50159500 \\
\hline $\mathrm{C}$ & -8.24679100 & -5.07498700 & 15500 \\
\hline $\mathrm{C}$ & -9.28669600 & -5.39808900 & 10.17490500 \\
\hline $\mathrm{C}$ & -9.74773900 & -7.08994700 & 8.35542600 \\
\hline $\mathrm{C}$ & -12.40657000 & -6.13038900 & 5.1347940 \\
\hline $\mathrm{C}$ & -10.36933000 & -7.13235600 & 5.9384330 \\
\hline $\mathrm{C}$ & -11.85463400 & -6.12027200 & 7.4 \\
\hline $\mathrm{C}$ & -12.72537700 & 5700 & 770 \\
\hline $\mathrm{C}$ & -11.20473800 & 0700 & 560 \\
\hline $\mathrm{C}$ & -13.30971100 & -5.75066900 & 9420 \\
\hline $\mathrm{N}$ & -12.97902000 & -6.01297000 & 2.8289630 \\
\hline $\mathrm{N}$ & -13.86095700 & -5.58879800 & 1.8465450 \\
\hline $\mathrm{C}$ & -13.54742000 & -5.78676200 & 2430 \\
\hline $\mathrm{C}$ & -15.06531500 & 1.79026700 & -10.4902470 \\
\hline $\mathrm{C}$ & -15.60718000 & 2.05968100 & -11. \\
\hline $\mathrm{C}$ & -7.13827900 & -2.20632200 & 10.36 \\
\hline $\mathrm{C}$ & -6.58662000 & -2.47086000 & 11.80380700 \\
\hline $\mathrm{C}$ & -5.96073700 & -1.79726500 & 9.44813800 \\
\hline $\mathrm{C}$ & -3.78754300 & -1.10937500 & 7.77228100 \\
\hline $\mathrm{C}$ & -4.79023000 & -2.57384300 & 9.42622800 \\
\hline $\mathrm{C}$ & -6.02 & -0.67 & 4400 \\
\hline $\mathrm{C}$ & -4.95 & -0.33 & 7.78202400 \\
\hline $\mathrm{C}$ & -3.71845300 & -2.23751500 & 8.59862200 \\
\hline $\mathrm{C}$ & -2.61487000 & -0.72935000 & 6.89293300 \\
\hline $\mathrm{C}$ & -3.73392400 & -1.20753800 & 2.78338800 \\
\hline $\mathrm{C}$ & -3.22130700 & 0.21092100 & 4.66031600 \\
\hline $\mathrm{C}$ & -3.08954700 & -2.14005400 & 4.90866200 \\
\hline $\mathrm{C}$ & -3.46133700 & -2.323 & 3.59290900 \\
\hline $\mathrm{C}$ & -3.60363600 & 0.07550400 & 3.34304100 \\
\hline $\mathrm{C}$ & -4.15459600 & -1.40521600 & 1.40080100 \\
\hline $\mathrm{N}$ & -4.46038900 & -0.37899200 & 0.67285200 \\
\hline $\mathrm{N}$ & -4.87077700 & -0.65025000 & -0.62462100 \\
\hline $\mathrm{C}$ & -5.24626600 & 0.37326500 & -1.43983100 \\
\hline $\mathrm{C}$ & -13.54350100 & 1.51426400 & -10.5 \\
\hline $\mathrm{C}$ & -10.75394500 & 1.06649600 & -10.6924370 \\
\hline $\mathrm{C}$ & -12.71387200 & 2.34471500 & -11.3342200 \\
\hline $\mathrm{C}$ & -12.94631400 & 0.46465900 & -9.8482980 \\
\hline $\mathrm{C}$ & -11.57124400 & 0.23826800 & -9.9151490 \\
\hline $\mathrm{C}$ & -11.33677900 & 2.12610200 & -11.39702700 \\
\hline $\mathrm{C}$ & -9.26293700 & 0.81744300 & -10.78428400 \\
\hline $\mathrm{C}$ & -7.50752600 & 1.37761400 & -6.91405200 \\
\hline $\mathrm{C}$ & -8.15512000 & -0.06863700 & -8.72753100 \\
\hline $\mathrm{C}$ & -8.51781100 & 2.26402900 & -8.91358300 \\
\hline $\mathrm{C}$ & -7.96970400 & 2.47288600 & -7.66480000 \\
\hline $\mathrm{C}$ & -7.60720400 & 0.09122200 & -7.47277800 \\
\hline $\mathrm{C}$ & -6.95671800 & 1.59596400 & -5.58071700 \\
\hline $\mathrm{N}$ & -6.57764800 & 0.57932300 & -4.87418100 \\
\hline $\mathrm{N}$ & -6.08984300 & 0.85989300 & -3.60670000 \\
\hline $\mathrm{C}$ & -5.69383100 & -0.16154700 & -2.79831200 \\
\hline $\mathrm{C}$ & -8.18576700 & -1.06700500 & 10.4267360 \\
\hline
\end{tabular}

\begin{tabular}{|c|c|c|}
\hline & & \\
\hline & 0.097 & \\
\hline .40077200 & .14384300 & \\
\hline 0.32729400 & -0.10196900 & 765590 \\
\hline 100 & 1.14 & 11.20875300 \\
\hline 1.05730100 & 2.18756600 & 10.57664700 \\
\hline & 3.89814700 & 667011800 \\
\hline 12.38346100 & 2.62643500 & 3600 \\
\hline 0.20613100 & 0000 & 4900 \\
\hline 63000 & 2900 & 73700 \\
\hline & & 98000 \\
\hline & & 1700 \\
\hline & & 3500 \\
\hline & 300 & 4500 \\
\hline & & 6600 \\
\hline 00 & & 7200 \\
\hline & 100 & 9900 \\
\hline & & \\
\hline & & \\
\hline & & 100 \\
\hline & & \\
\hline & & 500 \\
\hline & & 300 \\
\hline & & \\
\hline & & \\
\hline & & \\
\hline & & \\
\hline & & \\
\hline & & 100 \\
\hline & & 5800 \\
\hline & & \\
\hline & & \\
\hline & & \\
\hline & & \\
\hline & & \\
\hline & & 2000 \\
\hline & & 000 \\
\hline-8.6 & 0 & 00 \\
\hline & & \\
\hline & & \\
\hline & & \\
\hline & & \\
\hline & & 000 \\
\hline-15 . & 500 & 300 \\
\hline-16.1 & & 2000 \\
\hline & & \\
\hline & & \\
\hline & & \\
\hline & & \\
\hline & 00 & 00 \\
\hline & -4.2 & \\
\hline & & \\
\hline & & \\
\hline & & \\
\hline & & 600 \\
\hline & & \\
\hline & & \\
\hline & & \\
\hline & & \\
\hline & & \\
\hline & -7.8 & \\
\hline-9 . & -7.6 & \\
\hline & & 300 \\
\hline & & 5100 \\
\hline & & \\
\hline & & \\
\hline & & \\
\hline-1 & & 500 \\
\hline & & 900 \\
\hline & 3700 & 15800 \\
\hline & -1.62 & \\
\hline & & \\
\hline & & \\
\hline & & \\
\hline & -0.0 & \\
\hline & & \\
\hline & -2.84 & 1800 \\
\hline & -1.37210200 & 7.08025200 \\
\hline & & \\
\hline 3.10151800 & 1.17559200 & 5.13089300 \\
\hline
\end{tabular}




$\begin{array}{lcc}-2.88188900 & -2.96616700 & 5.57323900 \\ -3.54521200 & -3.32791500 & 3.20001100 \\ -3.80070200 & 0.95583100 & 2.74917500 \\ -4.20603900 & -2.43136300 & 1.04060900 \\ -4.91768000 & -1.61074700 & -0.98666900 \\ -13.13558200 & 3.17709100 & -11.88329200 \\ -13.56053400 & -0.18400500 & -9.23882000 \\ -11.13486600 & -0.58412100 & -9.35811900 \\ -10.71378900 & 2.77753200 & -12.00017500 \\ -8.78861100 & 1.51819100 & -11.47275200 \\ -9.03976000 & -0.20479100 & -11.09321300 \\ -8.24848600 & -1.03634900 & -9.19758200 \\ -8.89920600 & 3.07161000 & -9.52169700 \\ -7.90807000 & 3.47932200 & -7.27397500 \\ -7.26034900 & -0.77328600 & -6.92621200 \\ -6.89901600 & 2.62490800 & -5.22959000 \\ -6.04103000 & 1.82105200 & -3.24678600 \\ -6.98818600 & 0.20072500 & 11.70519200 \\ -9.62797600 & -2.02678800 & 9.14705900 \\ -11.26159900 & -0.18840200 & 9.23168500 \\ -8.61804500 & 2.03696100 & 11.78541000 \\ -10.72338200 & 2.97523800 & 11.25345000 \\ -12.04591600 & 1.84016200 & 10.87989900 \\ -13.16137700 & 2.05255400 & 8.98992600 \\ -9.32050000 & 3.65254500 & 9.28638900 \\ -9.48753900 & 4.66240900 & 7.01546000 \\ -13.45114500 & 2.98757700 & 6.69958300 \\ -10.75691100 & 5.03358900 & 4.95025900 \\ -11.94767900 & 5.30551100 & 2.97084300 \\ -17.37134000 & 3.28302600 & -10.28554300 \\ -13.38847800 & 3.07662200 & -8.68302500 \\ -13.85522300 & 5.03328700 & -7.26944000 \\ -17.83994300 & 5.23209600 & -8.86650000 \\ -17.18370600 & 6.86440200 & -7.29578500 \\ -15.45419500 & 7.29242200 & -7.28899500 \\ -14.26285200 & 7.13845100 & -5.41596700 \\ -17.89556500 & 5.11096000 & -5.71841700 \\ -17.79223200 & 4.42017400 & -3.32837400 \\ -14.02190800 & 6.49643200 & -3.02131300 \\ -16.62563900 & 4.39626700 & -1.18832000 \\ -15.49917600 & 4.31247600 & 0.82062800\end{array}$

\section{Complex C1•7b-1}

E -5753.971728 a.u. (zero imaginary freq.)

$\begin{array}{cccc}\text { E }-5753.971728 \text { a.u. (zero imaginary freq. } & \\ \mathrm{C} & -6.57087100 & 10.08108800 & -2.72168700 \\ \mathrm{C} & -5.72104300 & 7.40499100 & -2.38701000 \\ \mathrm{C} & -6.80687600 & 9.12067400 & -3.71726900 \\ \mathrm{C} & -5.91498500 & 9.67074700 & -1.55018500 \\ \mathrm{C} & -5.49915400 & 8.35250500 & -1.38168000 \\ \mathrm{C} & -6.38142200 & 7.79928900 & -3.55613300 \\ \mathrm{C} & -5.20734700 & 5.99356500 & -2.21745500 \\ \mathrm{C} & -8.20746400 & 3.32378100 & -0.74639200 \\ \mathrm{C} & -7.01430500 & 4.32224800 & -2.56994100 \\ \mathrm{C} & -6.50158600 & 4.97461900 & -0.34997600 \\ \mathrm{C} & -7.46350800 & 4.10224300 & 0.15588500 \\ \mathrm{C} & -7.98595200 & 3.44862800 & -2.11566300 \\ \mathrm{C} & -7.68349900 & 3.94224100 & 1.59268500 \\ \mathrm{~N} & -7.19675100 & 4.77374500 & 2.45537200 \\ \mathrm{~N} & -7.43884300 & 4.41783800 & 3.79051200 \\ \mathrm{C} & -7.08755900 & 5.22557500 & 4.81534700 \\ \mathrm{C} & -1.34053000 & 7.05730200 & 13.24441700 \\ \mathrm{C} & -4.01155600 & 6.38083000 & 13.87162400 \\ \mathrm{C} & -2.30800200 & 8.05254300 & 13.43718400 \\ \mathrm{C} & -1.72781200 & 5.71550200 & 13.40592700 \\ \mathrm{C} & -3.04314100 & 5.37961400 & 13.71722700 \\ \mathrm{C} & -3.63248100 & 7.71926100 & 13.73413700 \\ \mathrm{C} & -5.44397300 & 6.02230100 & 14.19795000 \\ \mathrm{C} & -7.06413200 & 3.57366400 & 11.08849000 \\ \mathrm{C} & -6.44353900 & 3.88604000 & 13.38565900 \\ \mathrm{C} & -6.14326000 & 5.66184400 & 11.84849000 \\ \mathrm{C} & -6.65024700 & 4.88965500 & 10.80905500 \\ \mathrm{C} & -6.96284800 & 3.07939200 & 12.38303100 \\ \mathrm{C} & -6.71894900 & 5.46108400 & 9.46343300 \\ \mathrm{~N} & -7.05376700 & 4.69211400 & 8.47775400 \\ \mathrm{~N} & -7.03366600 & 5.28266300 & 7.20777500 \\ \mathrm{C} & -7.33479000 & 4.50417900 & 6.14311300 \\ \mathrm{C} & -7.04342300 & 11.54756600 & -2.86656600 \\ \mathrm{C} & -7.28156300 & 11.91784500 & -4.36071100 \\ \mathrm{C} & 0.14839400 & 7.41231200 & 13.02397900 \\ \mathrm{C} & 0.75213700 & 7.47496300 & 14.45654000\end{array}$

\begin{tabular}{|c|c|c|c|}
\hline $\mathrm{C}$ & 0.85494000 & 6.34394900 & 12.14848700 \\
\hline $\mathrm{C}$ & 2.14478100 & 4.45847200 & 10.47882300 \\
\hline $\mathrm{C}$ & 2.12621500 & 5.84465900 & 12.47057400 \\
\hline $\mathrm{C}$ & 0.24212800 & 5.87763200 & 10.97317500 \\
\hline $\mathrm{C}$ & 0.87707000 & 4.95184600 & 10.14676600 \\
\hline $\mathrm{C}$ & 2.76198400 & 4.91087000 & 11.64963300 \\
\hline $\mathrm{C}$ & 2.81845200 & 3.43400300 & 9.59479500 \\
\hline $\mathrm{C}$ & 6.11459400 & 5.16369400 & 7.48482000 \\
\hline $\mathrm{C}$ & 5.26801300 & 3.70817400 & 9.19499800 \\
\hline $\mathrm{C}$ & 3.74868200 & 4.93166600 & 7.85244800 \\
\hline $\mathrm{C}$ & 4.79726900 & 5.51371100 & 7.14438800 \\
\hline $\mathrm{C}$ & 6.34353800 & 4.26056800 & 8.52020200 \\
\hline $\mathrm{C}$ & 4.54587700 & 6.47657600 & 6.07348800 \\
\hline $\mathrm{N}$ & 3.34768000 & 6.89929300 & 5.83660600 \\
\hline $\mathrm{N}$ & 3.23555900 & 7.81973800 & 4.79423300 \\
\hline $\mathrm{C}$ & 2.04076100 & 8.40010200 & 4.51418900 \\
\hline $\mathrm{C}$ & -5.94520600 & 12.45898200 & -2.26005400 \\
\hline $\mathrm{C}$ & -3.87648900 & 14.03714000 & -1.15024000 \\
\hline $\mathrm{C}$ & -4.77207400 & 12.72904900 & -2.98695800 \\
\hline $\mathrm{C}$ & -6.05200100 & 12.98013100 & -0.96290000 \\
\hline $\mathrm{C}$ & -5.02761100 & 13.75455100 & -0.41016000 \\
\hline $\mathrm{C}$ & -3.75656900 & 13.51466000 & -2.44558700 \\
\hline $\mathrm{C}$ & -2.80376300 & 14.93382900 & -0.57191600 \\
\hline $\mathrm{C}$ & 0.86425800 & 12.79134400 & -0.09240000 \\
\hline $\mathrm{C}$ & -0.45465800 & 14.43443200 & -1.23878100 \\
\hline $\mathrm{C}$ & -1.39640900 & 13.25622100 & 0.58777300 \\
\hline $\mathrm{C}$ & -0.21892500 & 12.54028000 & 0.77156300 \\
\hline $\mathrm{C}$ & 0.73804300 & 13.73920500 & -1.09942600 \\
\hline $\mathrm{C}$ & -0.13150100 & 11.55674300 & 1.85289600 \\
\hline $\mathrm{N}$ & 0.98720100 & 10.93906800 & 2.04212900 \\
\hline $\mathrm{N}$ & 1.02575700 & 10.00892200 & 3.07873700 \\
\hline $\mathrm{C}$ & 2.19733000 & 9.37670400 & 3.34849800 \\
\hline $\mathrm{C}$ & 0.32337200 & 8.75976200 & 12.27587600 \\
\hline $\mathrm{C}$ & 0.69133600 & 11.16678600 & 10.83219400 \\
\hline $\mathrm{C}$ & 1.33177800 & 9.66725400 & 12.62951100 \\
\hline $\mathrm{C}$ & -0.49132100 & 9.07826500 & 11.17358200 \\
\hline $\mathrm{C}$ & -0.31309100 & 10.26233800 & 10.46289200 \\
\hline $\mathrm{C}$ & 1.51418400 & 10.85681400 & 11.91824100 \\
\hline $\mathrm{C}$ & 0.86117900 & 12.46643300 & 10.07989500 \\
\hline $\mathrm{C}$ & -1.89715200 & 15.36458100 & 11.60073400 \\
\hline $\mathrm{C}$ & 0.21375800 & 14.22664800 & 11.71988600 \\
\hline $\mathrm{C}$ & -1.29952100 & 13.68980400 & 9.98158000 \\
\hline $\mathrm{C}$ & -2.22404000 & 14.61774000 & 10.45939200 \\
\hline $\mathrm{C}$ & -0.66947900 & 15.15969500 & 12.23113500 \\
\hline $\mathrm{C}$ & -3.49926300 & 14.81837300 & 9.77208600 \\
\hline $\mathrm{N}$ & -3.82326700 & 14.05632900 & 8.78010100 \\
\hline $\mathrm{N}$ & -5.03731100 & 14.34581300 & 8.15892000 \\
\hline $\mathrm{C}$ & -5.47780300 & 13.56598800 & 7.13798200 \\
\hline $\mathrm{C}$ & -8.38085200 & 11.75599100 & -2.11759500 \\
\hline $\mathrm{C}$ & -10.78047300 & 12.24367900 & -0.70963500 \\
\hline $\mathrm{C}$ & -9.03145300 & 10.72447700 & -1.43239000 \\
\hline $\mathrm{C}$ & -8.97570100 & 13.03250400 & -2.12173000 \\
\hline $\mathrm{C}$ & -10.15604200 & 13.27541400 & -1.42758600 \\
\hline $\mathrm{C}$ & -10.21974800 & 10.96446500 & -0.73405300 \\
\hline $\mathrm{C}$ & -11.99538300 & 12.53078900 & 0.13476400 \\
\hline $\mathrm{C}$ & -10.90184800 & 14.57823400 & 3.74385700 \\
\hline $\mathrm{C}$ & -12.40351000 & 14.25334500 & 1.89928900 \\
\hline $\mathrm{C}$ & -10.45582100 & 12.92037800 & 2.06117200 \\
\hline $\mathrm{C}$ & -10.07349600 & 13.56214400 & 3.23336900 \\
\hline $\mathrm{C}$ & -12.06971000 & 14.91705300 & 3.07061000 \\
\hline $\mathrm{C}$ & -8.81755100 & 13.17838900 & 3.87923600 \\
\hline $\mathrm{N}$ & -8.44085400 & 13.82327600 & 4.93553100 \\
\hline $\mathrm{N}$ & -7.22620900 & 13.42113400 & 5.50648000 \\
\hline $\mathrm{C}$ & -6.79411400 & 14.11245300 & 6.59045500 \\
\hline $\mathrm{N}$ & -6.28739400 & 5.05894700 & -1.68504700 \\
\hline $\mathrm{O}$ & -6.60691300 & 6.37258300 & 4.74151400 \\
\hline $\mathrm{O}$ & -7.73909900 & 3.32668900 & 6.16862300 \\
\hline $\mathrm{N}$ & -6.04362500 & 5.15286900 & 13.10324200 \\
\hline $\mathrm{N}$ & -0.11333200 & 13.51552100 & 10.60366600 \\
\hline $\mathrm{N}$ & -11.59745400 & 13.27067200 & 1.41853600 \\
\hline $\mathrm{N}$ & -1.49179900 & 14.18725500 & -0.39705600 \\
\hline $\mathrm{N}$ & 3.99599500 & 4.04455400 & 8.84450100 \\
\hline $\mathrm{O}$ & 3.28094400 & 9.54132900 & 2.76376600 \\
\hline $\mathrm{O}$ & 0.95763200 & 8.20918000 & 5.09067400 \\
\hline $\mathrm{O}$ & -7.35238400 & 15.09227000 & 7.12023800 \\
\hline $\mathrm{O}$ & -4.92069400 & 12.54558600 & 6.69605300 \\
\hline $\mathrm{H}$ & -7.31348300 & 9.39414500 & -4.63318000 \\
\hline $\mathrm{H}$ & -5.70985300 & 10.39030300 & -0.76806000 \\
\hline $\mathrm{H}$ & -4.97614000 & 8.07078000 & -0.47671600 \\
\hline $\mathrm{H}$ & -6.55342500 & 7.07991500 & -4.34958200 \\
\hline
\end{tabular}




\begin{tabular}{|c|c|c|c|}
\hline $\mathrm{H}$ & -4.38837700 & 5.95142500 & -1.49837600 \\
\hline $\mathrm{H}$ & -4.88468800 & 5.56591800 & -3.16674500 \\
\hline $\mathrm{H}$ & -8.94818300 & 2.62886700 & -0.37047100 \\
\hline $\mathrm{H}$ & -6.78187600 & 4.44436900 & -3.61721700 \\
\hline $\mathrm{H}$ & -5.88481600 & 5.58449400 & 0.29513200 \\
\hline $\mathrm{H}$ & -8.54825700 & 2.86566600 & -2.83052500 \\
\hline $\mathrm{H}$ & -8.27114100 & 3.07121800 & 1.88130900 \\
\hline $\mathrm{H}$ & -7.84085600 & 3.50173800 & 4.02984400 \\
\hline $\mathrm{H}$ & -2.03457000 & 9.09541700 & 13.34282000 \\
\hline $\mathrm{H}$ & -0.99370100 & 4.92903900 & 13.28520300 \\
\hline $\mathrm{H}$ & -3.31942400 & 4.33717700 & 13.83479100 \\
\hline $\mathrm{H}$ & -4.36966100 & 8.50402800 & 13.86354800 \\
\hline $\mathrm{H}$ & -6.06904900 & 6.91331400 & 14.27438100 \\
\hline $\mathrm{H}$ & -5.52053700 & 5.44180000 & 15.11818200 \\
\hline $\mathrm{H}$ & -7.45794800 & 2.96312100 & 10.28734100 \\
\hline $\mathrm{H}$ & -6.33821300 & 3.55064200 & 14.40680500 \\
\hline $\mathrm{H}$ & -5.80365300 & 6.67753800 & 11.69872100 \\
\hline $\mathrm{H}$ & -7.27593700 & 2.07431100 & 12.62631800 \\
\hline $\mathrm{H}$ & -6.48056000 & 6.51413700 & 9.34502800 \\
\hline $\mathrm{H}$ & -6.78444500 & 6.28501500 & 7.10237500 \\
\hline $\mathrm{H}$ & -7.47424800 & 12.99002700 & -4.44899400 \\
\hline $\mathrm{H}$ & -8.15033700 & 11.37955300 & -4.74922200 \\
\hline $\mathrm{H}$ & -6.41160600 & 11.66675100 & -4.97312500 \\
\hline $\mathrm{H}$ & 1.81976500 & 7.70502300 & 14.45517200 \\
\hline $\mathrm{H}$ & 0.60622700 & 6.51084700 & 14.95236800 \\
\hline $\mathrm{H}$ & 0.23173300 & 8.24688800 & 15.03105500 \\
\hline $\mathrm{H}$ & 2.63297600 & 6.17214700 & 13.36760900 \\
\hline $\mathrm{H}$ & -0.74606300 & 6.22708400 & 10.70393600 \\
\hline $\mathrm{H}$ & 0.38083000 & 4.60052800 & 9.24872700 \\
\hline $\mathrm{H}$ & 3.74033100 & 4.53175600 & 11.92527600 \\
\hline $\mathrm{H}$ & 2.13424500 & 3.05012600 & 8.83682000 \\
\hline $\mathrm{H}$ & 3.22815300 & 2.60379500 & 10.17102300 \\
\hline $\mathrm{H}$ & 6.94344400 & 5.60227500 & 6.94302600 \\
\hline $\mathrm{H}$ & 5.38697900 & 2.99828200 & 9.99974000 \\
\hline $\mathrm{H}$ & 2.71564100 & 5.16054200 & 7.63324500 \\
\hline $\mathrm{H}$ & 7.34651400 & 3.97955100 & 8.80705700 \\
\hline $\mathrm{H}$ & 5.41882200 & 6.81277000 & 5.51496400 \\
\hline $\mathrm{H}$ & 4.05066900 & 8.09293600 & 4.23169600 \\
\hline $\mathrm{H}$ & -4.64535500 & 12.32695000 & -3.98424200 \\
\hline $\mathrm{H}$ & -6.93978400 & 12.78386100 & -0.37689200 \\
\hline $\mathrm{H}$ & -5.13375500 & 14.15067900 & 0.59387700 \\
\hline $\mathrm{H}$ & -2.86806900 & 13.72344600 & -3.03196800 \\
\hline $\mathrm{H}$ & -3.08821000 & 15.30244800 & 0.41464300 \\
\hline $\mathrm{H}$ & -2.58731500 & 15.77957300 & -1.22659700 \\
\hline $\mathrm{H}$ & 1.78456300 & 12.23960000 & 0.04272300 \\
\hline $\mathrm{H}$ & -0.60294000 & 15.18877600 & -1.99705100 \\
\hline $\mathrm{H}$ & -2.26582300 & 13.11313900 & 1.21496200 \\
\hline $\mathrm{H}$ & 1.55244200 & 13.95167600 & -1.77681200 \\
\hline $\mathrm{H}$ & -1.02358900 & 11.39215500 & 2.45460100 \\
\hline $\mathrm{H}$ & 0.19925000 & 9.79334200 & 3.64839700 \\
\hline $\mathrm{H}$ & 1.99038300 & 9.45949700 & 13.46131800 \\
\hline $\mathrm{H}$ & -1.28141000 & 8.40253400 & 10.87439200 \\
\hline $\mathrm{H}$ & -0.95546200 & 10.48534600 & 9.61774800 \\
\hline $\mathrm{H}$ & 2.30275100 & 11.54149400 & 12.21077500 \\
\hline $\mathrm{H}$ & 0.64378200 & 12.35371100 & 9.01731900 \\
\hline $\mathrm{H}$ & 1.86052400 & 12.88276000 & 10.21121600 \\
\hline $\mathrm{H}$ & -2.59789000 & 16.09487200 & 11.98619200 \\
\hline $\mathrm{H}$ & 1.17892600 & 14.02695100 & 12.16215800 \\
\hline $\mathrm{H}$ & -1.49647600 & 13.09771000 & 9.10033000 \\
\hline $\mathrm{H}$ & -0.39234500 & 15.71994500 & 13.11247900 \\
\hline $\mathrm{H}$ & -4.12540900 & 15.63056200 & 10.13884700 \\
\hline $\mathrm{H}$ & -5.61275800 & 15.14833900 & 8.44286700 \\
\hline $\mathrm{H}$ & -8.60548700 & 9.73106500 & -1.42407900 \\
\hline $\mathrm{H}$ & -8.49794500 & 13.84792800 & -2.65113200 \\
\hline $\mathrm{H}$ & -10.58791400 & 14.27013300 & -1.42897500 \\
\hline $\mathrm{H}$ & -10.70100600 & 10.15558800 & -0.19486300 \\
\hline $\mathrm{H}$ & -12.49250100 & 11.61190000 & 0.45050800 \\
\hline $\mathrm{H}$ & -12.70736000 & 13.18177500 & -0.37308900 \\
\hline $\mathrm{H}$ & -10.61310700 & 15.08796100 & 4.65303600 \\
\hline $\mathrm{H}$ & -13.29226400 & 14.48562600 & 1.33118600 \\
\hline $\mathrm{H}$ & -9.86070600 & 12.13614300 & 1.61648800 \\
\hline $\mathrm{H}$ & -12.72198800 & 15.69673900 & 3.43692000 \\
\hline $\mathrm{H}$ & -8.24231800 & 12.37212000 & 3.43069000 \\
\hline $\mathrm{H}$ & -6.68924800 & 12.62687200 & 5.08246900 \\
\hline $\mathrm{C}$ & -3.35492700 & 9.96445300 & 1.27981200 \\
\hline $\mathrm{C}$ & -3.12321700 & 8.58943300 & 1.16046000 \\
\hline $\mathrm{C}$ & -3.83816800 & 7.69259100 & 1.96060300 \\
\hline $\mathrm{C}$ & -4.75975900 & 8.16076100 & 2.90041800 \\
\hline $\mathrm{C}$ & -4.96064700 & 9.54089500 & 3.05141600 \\
\hline $\mathrm{C}$ & -4.26954900 & 10.43604400 & 2.22024400 \\
\hline
\end{tabular}

$\begin{array}{llrc}\mathrm{C} & -5.84269500 & 10.15572800 & 4.09148500 \\ \mathrm{~N} & -6.30744500 & 9.38745100 & 5.09373100 \\ \mathrm{O} & -6.09155800 & 11.40403700 & 4.04313600 \\ \mathrm{C} & -7.03318000 & 10.02729500 & 6.19389100 \\ \mathrm{C} & -7.18280100 & 9.06262500 & 7.35767200 \\ \mathrm{~N} & -8.01179300 & 9.54580300 & 8.31783100 \\ \mathrm{O} & -6.58148000 & 7.96777500 & 7.42537300 \\ \mathrm{~N} & -8.30708100 & 8.77811200 & 9.46052100 \\ \mathrm{C} & -9.08527500 & 9.35749600 & 10.31653300 \\ \mathrm{C} & -9.52565100 & 8.67137900 & 11.53093600 \\ \mathrm{C} & -10.21694700 & 9.39054900 & 12.51673900 \\ \mathrm{C} & -10.63175400 & 8.72664000 & 13.66803600 \\ \mathrm{~N} & -10.40618000 & 7.41030200 & 13.89212100 \\ \mathrm{C} & -9.74265700 & 6.72417600 & 12.92630600 \\ \mathrm{C} & -9.29249800 & 7.30384100 & 11.74712900 \\ \mathrm{H} & -2.82313400 & 10.66334500 & 0.64564000 \\ \mathrm{H} & -2.40284900 & 8.21957400 & 0.44000800 \\ \mathrm{H} & -3.68044700 & 6.62542400 & 1.85301200 \\ \mathrm{H} & -5.33119200 & 7.44438900 & 3.47701200 \\ \mathrm{H} & -4.45305400 & 11.49691600 & 2.33195400 \\ \mathrm{H} & -6.17235000 & 8.37540800 & 5.14591100 \\ \mathrm{H} & -8.01838600 & 10.38441900 & 5.87442500 \\ \mathrm{H} & -6.44934900 & 10.88634900 & 6.54429600 \\ \mathrm{H} & -8.44795800 & 10.46788500 & 8.20900500 \\ \mathrm{H} & -9.45520700 & 10.37456700 & 10.17970400 \\ \mathrm{H} & -10.42367800 & 10.44594400 & 12.38674500 \\ \mathrm{H} & -11.16481200 & 9.26757300 & 14.44093500 \\ \mathrm{H} & -9.57114900 & 5.66977000 & 13.10956100 \\ \mathrm{H} & -8.78293700 & 6.71356700 & 10.99963200\end{array}$

\section{Complex C1•7b -2}

\begin{tabular}{|c|c|c|c|}
\hline \multicolumn{4}{|c|}{ E -5753.97152 a.u. (zero imaginary freq.) } \\
\hline $\mathrm{C}$ & -6.48452800 & 10.08396800 & -2.63075500 \\
\hline $\mathrm{C}$ & -5.56687300 & 7.44840400 & -2.18468500 \\
\hline $\mathrm{C}$ & -6.57078400 & 9.11313800 & -3.63804300 \\
\hline $\mathrm{C}$ & -5.94169900 & 9.70626500 & -1.39070100 \\
\hline $\mathrm{C}$ & -5.49441400 & 8.40773900 & -1.16677200 \\
\hline $\mathrm{C}$ & -6.11041500 & 7.81044900 & -3.42078300 \\
\hline $\mathrm{C}$ & -5.02223900 & 6.05854400 & -1.95010200 \\
\hline $\mathrm{C}$ & -8.04998500 & 3.36017600 & -0.59232200 \\
\hline $\mathrm{C}$ & -6.80137100 & 4.37225300 & -2.37132200 \\
\hline $\mathrm{C}$ & -6.36244800 & 5.01302100 & -0.13232500 \\
\hline $\mathrm{C}$ & -7.33871600 & 4.13634800 & 0.33729800 \\
\hline $\mathrm{C}$ & -7.78268900 & 3.49166800 & -1.95313800 \\
\hline $\mathrm{C}$ & -7.60723500 & 3.97792200 & 1.76560500 \\
\hline $\mathrm{N}$ & -7.13829500 & 4.80687700 & 2.64045200 \\
\hline $\mathrm{N}$ & -7.42574900 & 4.46270200 & 3.96862500 \\
\hline $\mathrm{C}$ & -7.08662000 & 5.26985800 & 4.99803800 \\
\hline $\mathrm{C}$ & -1.32299300 & 7.04295000 & 13.20558800 \\
\hline $\mathrm{C}$ & -3.96315100 & 6.37543100 & 13.96011600 \\
\hline $\mathrm{C}$ & -2.23827900 & 8.03773400 & 13.57842700 \\
\hline $\mathrm{C}$ & -1.74331800 & 5.70348100 & 13.25531800 \\
\hline $\mathrm{C}$ & -3.04288600 & 5.37105000 & 13.63220900 \\
\hline $\mathrm{C}$ & -3.54902600 & 7.71074200 & 13.93472200 \\
\hline $\mathrm{C}$ & -5.38343800 & 6.02589500 & 14.34913700 \\
\hline $\mathrm{C}$ & -7.11676800 & 3.61960900 & 11.27206200 \\
\hline $\mathrm{C}$ & -6.45003800 & 3.91563500 & 13.55877900 \\
\hline $\mathrm{C}$ & -6.14755900 & 5.68951100 & 12.02007200 \\
\hline $\mathrm{C}$ & -6.68097600 & 4.92743700 & 10.98719200 \\
\hline $\mathrm{C}$ & -7.00344400 & 3.12136300 & 12.56416700 \\
\hline $\mathrm{C}$ & -6.74672100 & 5.49891800 & 9.64191900 \\
\hline $\mathrm{N}$ & -7.11492700 & 4.74089300 & 8.65960900 \\
\hline $\mathrm{N}$ & -7.07599200 & 5.33125700 & 7.38994100 \\
\hline $\mathrm{C}$ & -7.38543500 & 4.56134400 & 6.32192600 \\
\hline $\mathrm{C}$ & -6.97262000 & 11.53831600 & -2.83435500 \\
\hline $\mathrm{C}$ & -7.27168000 & 11.83915000 & -4.33170100 \\
\hline $\mathrm{C}$ & 0.15351000 & 7.38976300 & 12.90177400 \\
\hline $\mathrm{C}$ & 0.84923600 & 7.40644500 & 14.29331000 \\
\hline $\mathrm{C}$ & 0.78261200 & 6.33325100 & 11.95583000 \\
\hline $\mathrm{C}$ & 1.89370300 & 4.43385200 & 10.17650200 \\
\hline $\mathrm{C}$ & 1.96608800 & 5.65179800 & 12.27513500 \\
\hline $\mathrm{C}$ & 0.16483000 & 6.04091700 & 10.72742300 \\
\hline $\mathrm{C}$ & 0.71107300 & 5.10876800 & 9.84772100 \\
\hline $\mathrm{C}$ & 2.51464400 & 4.71279900 & 11.39771600 \\
\hline $\mathrm{C}$ & 2.46870400 & 3.40196500 & 9.23323500 \\
\hline $\mathrm{C}$ & 5.96595500 & 4.89229700 & 7.27026600 \\
\hline $\mathrm{C}$ & 4.94225800 & 3.45708400 & 8.90024300 \\
\hline $\mathrm{C}$ & 3.58123700 & 4.87297400 & 7.57847400 \\
\hline $\mathrm{C}$ & 4.69668000 & 5.37743000 & 6.91397600 \\
\hline $\mathrm{C}$ & 6.08142300 & 3.93118100 & 8.27260800 \\
\hline
\end{tabular}




\begin{tabular}{|c|c|c|c|c|c|c|c|}
\hline $\mathrm{C}$ & 4.55261300 & 6.38552400 & 5.86310100 & $\mathrm{H}$ & -5.99478800 & 6.92233400 & 14.46454500 \\
\hline $\mathrm{N}$ & 3.39199700 & 6.89116900 & 5.60391700 & $\mathrm{H}$ & -5.42527100 & 5.43601600 & 15.26597200 \\
\hline $\mathrm{N}$ & 3.35172000 & 7.82972300 & 4.57322500 & $\mathrm{H}$ & -7.53252300 & 3.01663300 & 10.47625200 \\
\hline $\mathrm{C}$ & 2.18377800 & 8.45355700 & 4.27184600 & $\mathrm{H}$ & -6.32716200 & 3.57433300 & 14.57605400 \\
\hline $\mathrm{C}$ & -5.84423600 & 12.46682700 & -2.31409300 & $\mathrm{H}$ & -5.79088200 & 6.69883800 & 11.86636700 \\
\hline $\mathrm{C}$ & -3.71280300 & 14.05954200 & -1.35313800 & $\mathrm{H}$ & -7.32914000 & 2.12111100 & 12.81077100 \\
\hline $\mathrm{C}$ & -4.69562000 & 12.68703100 & -3.09568800 & $\mathrm{H}$ & -6.47167100 & 6.54225900 & 9.51916700 \\
\hline $\mathrm{C}$ & -5.89424200 & 13.04565000 & -1.03824700 & $\mathrm{H}$ & -6.80189000 & 6.32789600 & 7.28782600 \\
\hline $\mathrm{C}$ & -4.83922600 & 13.82770200 & -0.55948800 & $\mathrm{H}$ & -7.51209100 & 12.89887300 & -4.44982500 \\
\hline $\mathrm{C}$ & -3.64918800 & 13.47923100 & -2.62795700 & $\mathrm{H}$ & -8.13016800 & 11.25092300 & -4.66877800 \\
\hline $\mathrm{C}$ & -2.60601000 & 14.96286500 & -0.85582400 & $\mathrm{H}$ & -6.41452300 & 11.60251500 & -4.96734900 \\
\hline $\mathrm{C}$ & 1.06305000 & 12.81196100 & -0.40681900 & $\mathrm{H}$ & 1.92083900 & 7.60700900 & 14.22392700 \\
\hline $\mathrm{C}$ & -0.26741600 & 14.44742400 & -1.55230800 & $\mathrm{H}$ & 0.70913000 & 6.43776900 & 14.78211200 \\
\hline $\mathrm{C}$ & -1.19249100 & 13.27975500 & 0.28816200 & $\mathrm{H}$ & 0.38729500 & 8.18063200 & 14.91277800 \\
\hline $\mathrm{C}$ & -0.01280700 & 12.56600800 & 0.46755700 & $\mathrm{H}$ & 2.46986400 & 5.83635600 & 13.21397900 \\
\hline $\mathrm{C}$ & 0.92642200 & 13.75232200 & -1.41967300 & $\mathrm{H}$ & -0.75890700 & 6.53536600 & 10.45623300 \\
\hline $\mathrm{C}$ & 0.08050500 & 11.59379300 & 1.55773200 & $\mathrm{H}$ & 0.21167800 & 4.89456800 & 8.90923400 \\
\hline $\mathrm{N}$ & 1.19885700 & 10.97810400 & 1.75817700 & $\mathrm{H}$ & 3.42622500 & 4.19244800 & 11.67187300 \\
\hline $\mathrm{N}$ & 1.22331400 & 10.06234200 & 2.80878800 & $\mathrm{H}$ & 1.75988800 & 3.14509900 & 8.44539000 \\
\hline $\mathrm{C}$ & 2.38090200 & 9.41351400 & 3.09888300 & $\mathrm{H}$ & 2.77644500 & 2.49707500 & 9.75802000 \\
\hline $\mathrm{C}$ & 0.29988700 & 8.75799900 & 12.18600500 & $\mathrm{H}$ & 6.84744700 & 5.26653900 & 6.76455900 \\
\hline $\mathrm{C}$ & 0.62317600 & 11.20192400 & 10.79339700 & $\mathrm{H}$ & 4.96962600 & 2.70374700 & 9.67372400 \\
\hline $\mathrm{C}$ & 1.40139100 & 9.58994700 & 12.43170400 & $\mathrm{H}$ & 2.58062700 & 5.20697900 & 7.34576500 \\
\hline $\mathrm{C}$ & -0.63376300 & 9.17254600 & 11.21775100 & $\mathrm{H}$ & 7.04546800 & 3.54330000 & 8.56821700 \\
\hline $\mathrm{C}$ & -0.47471700 & 10.37247200 & 10.52924000 & $\mathrm{H}$ & 5.46319400 & 6.66631000 & 5.33532800 \\
\hline $\mathrm{C}$ & 1.55983000 & 10.79912700 & 11.74867500 & $\mathrm{H}$ & 4.18831600 & 8.06357200 & 4.02502900 \\
\hline $\mathrm{C}$ & 0.78506600 & 12.51530600 & 10.06176600 & $\mathrm{H}$ & -4.61075000 & 12.23650000 & -4.07643000 \\
\hline $\mathrm{C}$ & -1.92013900 & 15.39776100 & 11.70082100 & $\mathrm{H}$ & -6.76254700 & 12.89106700 & -0.41200600 \\
\hline $\mathrm{C}$ & 0.18364600 & 14.23950300 & 11.75601100 & $\mathrm{H}$ & -4.90219700 & 14.26827700 & 0.42950600 \\
\hline $\mathrm{C}$ & -1.36279600 & 13.76124400 & 10.03000900 & $\mathrm{H}$ & -2.77960400 & 13.64751200 & -3.25455200 \\
\hline $\mathrm{C}$ & -2.27333200 & 14.68136900 & 10.54831200 & $\mathrm{H}$ & -2.84530000 & 15.37719500 & 0.12473000 \\
\hline $\mathrm{C}$ & -0.68275000 & 15.16756300 & 12.30401300 & $\mathrm{H}$ & -2.40456400 & 15.77724900 & -1.55342900 \\
\hline $\mathrm{C}$ & -3.56247500 & 14.89415500 & 9.88949900 & $\mathrm{H}$ & 1.98526500 & 12.26218700 & -0.27628300 \\
\hline $\mathrm{N}$ & -3.90937400 & 14.12694100 & 8.90940500 & $\mathrm{H}$ & -0.42205800 & 15.19665000 & -2.31433100 \\
\hline $\mathrm{N}$ & -5.13052000 & 14.41155000 & 8.30245400 & $\mathrm{H}$ & -2.05765200 & 13.13681800 & 0.92133800 \\
\hline $\mathrm{C}$ & -5.58288600 & 13.61413900 & 7.29970300 & $\mathrm{H}$ & 1.73411100 & 13.95992800 & -2.10652900 \\
\hline $\mathrm{C}$ & -8.28945100 & 11.78381000 & -2.06062600 & $\mathrm{H}$ & -0.81148600 & 11.43507900 & 2.16105700 \\
\hline $\mathrm{C}$ & -10.69338400 & 12.32099400 & -0.67553300 & $\mathrm{H}$ & 0.38916400 & 9.86286100 & 3.37364000 \\
\hline $\mathrm{C}$ & -8.98095600 & 10.76158000 & -1.40180500 & $\mathrm{H}$ & 2.15261900 & 9.30615300 & 13.15550300 \\
\hline $\mathrm{C}$ & -8.84523700 & 13.07790800 & -2.04906600 & $\mathrm{H}$ & -1.49893300 & 8.55807400 & 11.00664900 \\
\hline $\mathrm{C}$ & -10.02700100 & 13.34538600 & -1.36608600 & $\mathrm{H}$ & -1.20868900 & 10.66741100 & 9.78687700 \\
\hline $\mathrm{C}$ & -10.16987800 & 11.02649500 & -0.71351500 & $\mathrm{H}$ & 2.41987800 & 11.42568700 & 11.95815200 \\
\hline $\mathrm{C}$ & -11.92446000 & 12.62447500 & 0.14003200 & $\mathrm{H}$ & 0.54221800 & 12.42455600 & 9.00240500 \\
\hline $\mathrm{C}$ & -10.91915800 & 14.66033500 & 3.77964500 & $\mathrm{H}$ & 1.79180600 & 12.91846700 & 10.17647400 \\
\hline $\mathrm{C}$ & -12.36198000 & 14.35599300 & 1.88614100 & $\mathrm{H}$ & -2.60808900 & 16.12295400 & 12.11765600 \\
\hline $\mathrm{C}$ & -10.44101600 & 12.99389700 & 2.11364800 & $\mathrm{H}$ & 1.15340000 & 14.01832000 & 12.17687500 \\
\hline $\mathrm{C}$ & -10.09002300 & 13.63049600 & 3.29859600 & $\mathrm{H}$ & -1.57982000 & 13.19526800 & 9.13629200 \\
\hline $\mathrm{C}$ & -12.05797300 & 15.01656600 & 3.06715800 & $\mathrm{H}$ & -0.38607200 & 15.70364700 & 13.19401500 \\
\hline $\mathrm{C}$ & -8.86300600 & 13.22999900 & 3.98818900 & $\mathrm{H}$ & -4.17594300 & 15.71169700 & 10.26530000 \\
\hline $\mathrm{N}$ & -8.51227700 & 13.87389300 & 5.05434800 & $\mathrm{H}$ & -5.69799400 & 15.22277400 & 8.57687600 \\
\hline $\mathrm{N}$ & -7.31862600 & 13.46018800 & 5.66034200 & $\mathrm{H}$ & -8.58851200 & 9.75440300 & -1.40837800 \\
\hline $\mathrm{C}$ & -6.89346200 & 14.16159500 & 6.74031500 & $\mathrm{H}$ & -8.33766000 & 13.88514600 & -2.56325600 \\
\hline $\mathrm{N}$ & -6.10664000 & 5.10701400 & -1.45885900 & $\mathrm{H}$ & -10.42988300 & 14.35219500 & -1.35923400 \\
\hline $\mathrm{O}$ & -6.58006100 & 6.40611900 & 4.93091700 & $\mathrm{H}$ & -10.68368700 & 10.22306300 & -0.19678300 \\
\hline $\mathrm{O}$ & -7.82641500 & 3.39686800 & 6.34155500 & $\mathrm{H}$ & -12.43922800 & 11.71104800 & 0.44340700 \\
\hline $\mathrm{N}$ & -6.03610000 & 5.17683200 & 13.27252200 & $\mathrm{H}$ & -12.61681700 & 13.28143500 & -0.38717800 \\
\hline $\mathrm{N}$ & -0.16792000 & 13.56089700 & 10.62691800 & $\mathrm{H}$ & -10.65366500 & 15.16639200 & 4.69790500 \\
\hline $\mathrm{N}$ & -11.55492600 & 13.36128100 & 1.43274600 & $\mathrm{H}$ & -13.22833300 & 14.59994700 & 1.28917300 \\
\hline $\mathrm{N}$ & -1.29683100 & 14.20580800 & -0.69998700 & $\mathrm{H}$ & -9.84291500 & 12.20089800 & 1.68876900 \\
\hline $\mathrm{N}$ & 3.71731400 & 3.92836500 & 8.53737400 & $\mathrm{H}$ & -12.71059000 & 15.80642600 & 3.41027300 \\
\hline $\mathrm{O}$ & 3.47435800 & 9.54752300 & 2.52539600 & $\mathrm{H}$ & -8.28386000 & 12.41499500 & 3.56110900 \\
\hline $\mathrm{O}$ & 1.08754900 & 8.30362000 & 4.83597800 & $\mathrm{H}$ & -6.77880700 & 12.65952100 & 5.25164700 \\
\hline $\mathrm{O}$ & -7.44804800 & 15.15221100 & 7.25354300 & $\mathrm{C}$ & -3.34775500 & 10.08446700 & 1.51355700 \\
\hline $\mathrm{O}$ & -5.03445400 & 12.58084400 & 6.87776900 & $\mathrm{C}$ & -3.09784600 & 8.71456300 & 1.37579900 \\
\hline $\mathrm{H}$ & -6.98181300 & 9.36310900 & -4.60675200 & $\mathrm{C}$ & -3.81286500 & 7.79623900 & 2.15150400 \\
\hline $\mathrm{H}$ & -5.84835400 & 10.43920500 & -0.59904100 & $\mathrm{C}$ & -4.75490300 & 8.23753400 & 3.08441800 \\
\hline $\mathrm{H}$ & -5.05922000 & 8.14792200 & -0.20995000 & $\mathrm{C}$ & -4.97636100 & 9.61273600 & 3.25080400 \\
\hline $\mathrm{H}$ & -6.16340700 & 7.08224500 & -4.22297500 & $\mathrm{C}$ & -4.28342400 & 10.52916900 & 2.44547500 \\
\hline $\mathrm{H}$ & -4.24730900 & 6.05868600 & -1.18268700 & $\mathrm{C}$ & -5.88771500 & 10.20342900 & 4.27852000 \\
\hline $\mathrm{H}$ & -4.62964400 & 5.61923500 & -2.86710300 & $\mathrm{~N}$ & -6.34901100 & 9.42197400 & 5.27098600 \\
\hline $\mathrm{H}$ & -8.80167200 & 2.66239300 & -0.24463700 & $\mathrm{O}$ & -6.16618700 & 11.44521500 & 4.22732500 \\
\hline $\mathrm{H}$ & -6.53725100 & 4.50264700 & -3.41034800 & $\mathrm{C}$ & -7.12067500 & 10.03726100 & 6.35533400 \\
\hline $\mathrm{H}$ & -5.76921700 & 5.62123900 & 0.53580100 & $\mathrm{C}$ & -7.27442200 & 9.05540700 & 7.50420400 \\
\hline $\mathrm{H}$ & -8.31844100 & 2.91008400 & -2.68925600 & $\mathrm{~N}$ & -8.19901700 & 9.46025000 & 8.41098000 \\
\hline $\mathrm{H}$ & -8.21315000 & 3.11408700 & 2.03684700 & $\mathrm{O}$ & -6.59205600 & 8.01022700 & 7.60354900 \\
\hline $\mathrm{H}$ & -7.85328200 & 3.55665700 & 4.20215200 & $\mathrm{~N}$ & -8.49069200 & 8.66220900 & 9.53331400 \\
\hline $\mathrm{H}$ & -1.93613700 & 9.07716200 & 13.57397400 & $\mathrm{C}$ & -9.36244500 & 9.16228700 & 10.34797600 \\
\hline $\mathrm{H}$ & -1.04861600 & 4.91524500 & 12.99621200 & $\mathrm{C}$ & -9.79512200 & 8.42587400 & 11.53616800 \\
\hline $\mathrm{H}$ & -3.34358000 & 4.32928400 & 13.66132900 & $\mathrm{C}$ & -10.60765400 & 9.05962000 & 12.48752300 \\
\hline $\mathrm{H}$ & -4.24918100 & 8.49700800 & 14.19467200 & $\mathrm{C}$ & -11.00425100 & 8.34684000 & 13.61627100 \\
\hline
\end{tabular}




$\begin{array}{llrc}\mathrm{N} & -10.64901600 & 7.06117100 & 13.85009700 \\ \mathrm{C} & -9.86928400 & 6.45707300 & 12.91667800 \\ \mathrm{C} & -9.42796300 & 7.08973200 & 11.76238100 \\ \mathrm{H} & -2.81398500 & 10.80038800 & 0.90027000 \\ \mathrm{H} & -2.36354100 & 8.36464500 & 0.65957800 \\ \mathrm{H} & -3.63925400 & 6.73300200 & 2.02980100 \\ \mathrm{H} & -5.32367400 & 7.50547200 & 3.64428400 \\ \mathrm{H} & -4.48201300 & 11.58510500 & 2.57345000 \\ \mathrm{H} & -6.18123500 & 8.41505900 & 5.32647700 \\ \mathrm{H} & -8.10366900 & 10.37388700 & 6.00920800 \\ \mathrm{H} & -6.56647500 & 10.90709300 & 6.72738600 \\ \mathrm{H} & -8.70780700 & 10.34122500 & 8.27758400 \\ \mathrm{H} & -9.81541800 & 10.14295500 & 10.19631400 \\ \mathrm{H} & -10.92087500 & 10.08734500 & 12.34978600 \\ \mathrm{H} & -11.63016600 & 8.82277800 & 14.36184100 \\ \mathrm{H} & -9.59405800 & 5.42600500 & 13.10626400 \\ \mathrm{H} & -8.81989100 & 6.56153900 & 11.04344900\end{array}$

Complex C1•7b -3

\begin{tabular}{|c|c|c|c|}
\hline \multicolumn{4}{|c|}{ E -5753.971074 a.u. (zero imaginary freq.) } \\
\hline $\mathrm{C}$ & -6.89186000 & 9.66817000 & -3.47176100 \\
\hline $\mathrm{C}$ & -7.31280600 & 7.11412500 & -2.33854900 \\
\hline $\mathrm{C}$ & -7.20121100 & 8.56438100 & -4.28016200 \\
\hline $\mathrm{C}$ & -6.81357000 & 9.47196400 & -2.08298100 \\
\hline $\mathrm{C}$ & -7.02299100 & 8.21383900 & -1.52188200 \\
\hline $\mathrm{C}$ & -7.40893200 & 7.30119000 & -3.72139500 \\
\hline $\mathrm{C}$ & -7.47059500 & 5.73781200 & -1.73665000 \\
\hline $\mathrm{C}$ & -11.24875000 & 5.15828400 & 0.18051900 \\
\hline $\mathrm{C}$ & -9.94426700 & 5.51915500 & -1.80113800 \\
\hline $\mathrm{C}$ & -8.86593400 & 5.46426900 & 0.30589300 \\
\hline $\mathrm{C}$ & -10.08162600 & 5.24433100 & 0.95254600 \\
\hline $\mathrm{C}$ & -11.17387200 & 5.31542300 & -1.20414100 \\
\hline $\mathrm{C}$ & -10.12505100 & 5.02879100 & 2.39902400 \\
\hline $\mathrm{N}$ & -9.12859800 & 5.37785200 & 3.14519000 \\
\hline $\mathrm{N}$ & -9.23456000 & 4.98202300 & 4.48606200 \\
\hline $\mathrm{C}$ & -8.33857800 & 5.40014600 & 5.40552400 \\
\hline $\mathrm{C}$ & -1.39674200 & 6.08493400 & 13.56180100 \\
\hline $\mathrm{C}$ & -3.64794900 & 4.41287200 & 13.91613600 \\
\hline $\mathrm{C}$ & -2.40948700 & 6.42171300 & 14.47471400 \\
\hline $\mathrm{C}$ & -1.53317700 & 4.90036800 & 12.82549800 \\
\hline $\mathrm{C}$ & -2.64133500 & 4.06646400 & 13.00571700 \\
\hline $\mathrm{C}$ & -3.52121900 & 5.60295200 & 14.64551700 \\
\hline $\mathrm{C}$ & -4.80346300 & 3.49276100 & 14.22324300 \\
\hline $\mathrm{C}$ & -7.51488700 & 2.60003400 & 11.03566100 \\
\hline $\mathrm{C}$ & -6.44495700 & 2.04284300 & 13.10136500 \\
\hline $\mathrm{C}$ & -5.88587200 & 4.07660900 & 12.01846200 \\
\hline $\mathrm{C}$ & -6.77370000 & 3.79482700 & 10.98041500 \\
\hline $\mathrm{C}$ & -7.35028200 & 1.72804800 & 12.10331800 \\
\hline $\mathrm{C}$ & -6.89710800 & 4.69170600 & 9.82275200 \\
\hline $\mathrm{N}$ & -7.66882200 & 4.29723500 & 8.86065500 \\
\hline $\mathrm{N}$ & -7.72181800 & 5.07237800 & 7.70019900 \\
\hline $\mathrm{C}$ & -8.54571300 & 4.64005700 & 6.71543300 \\
\hline $\mathrm{C}$ & -6.54120900 & 11.03908200 & -4.09374600 \\
\hline $\mathrm{C}$ & -7.32368500 & 11.25777400 & -5.42701400 \\
\hline $\mathrm{C}$ & -0.13725000 & 6.97923900 & 13.49350800 \\
\hline $\mathrm{C}$ & 0.61243500 & 6.75921100 & 14.84402100 \\
\hline $\mathrm{C}$ & 0.77268800 & 6.59615000 & 12.29650400 \\
\hline $\mathrm{C}$ & 2.46715600 & 5.96077900 & 10.11826200 \\
\hline $\mathrm{C}$ & 1.85882600 & 5.72241600 & 12.45611100 \\
\hline $\mathrm{C}$ & 0.53082300 & 7.11430500 & 11.01147600 \\
\hline $\mathrm{C}$ & 1.36275600 & 6.80360100 & 9.93829600 \\
\hline $\mathrm{C}$ & 2.69869600 & 5.41167800 & 11.38252200 \\
\hline $\mathrm{C}$ & 3.41117100 & 5.67962800 & 8.97061200 \\
\hline $\mathrm{C}$ & 5.79822700 & 9.16933000 & 8.31797300 \\
\hline $\mathrm{C}$ & 5.22910700 & 7.24069600 & 9.62860600 \\
\hline $\mathrm{C}$ & 4.07601100 & 7.65044900 & 7.60126000 \\
\hline $\mathrm{C}$ & 4.82548000 & 8.80582900 & 7.37555600 \\
\hline $\mathrm{C}$ & 5.99989300 & 8.37312200 & 9.44603800 \\
\hline $\mathrm{C}$ & 4.60146400 & 9.62701200 & 6.18649700 \\
\hline $\mathrm{N}$ & 3.78261500 & 9.23334600 & 5.26795300 \\
\hline $\mathrm{N}$ & 3.62580500 & 10.09575700 & 4.18290600 \\
\hline $\mathrm{C}$ & 2.84362800 & 9.72570800 & 3.13712800 \\
\hline $\mathrm{C}$ & -5.03042900 & 11.08640800 & -4.43679700 \\
\hline $\mathrm{C}$ & -2.29774600 & 11.21752000 & -5.15425600 \\
\hline $\mathrm{C}$ & -4.49359200 & 12.24045400 & -5.03892100 \\
\hline $\mathrm{C}$ & -4.17402900 & 10.00233600 & -4.20839600 \\
\hline $\mathrm{C}$ & -2.82468100 & 10.06392200 & -4.56793000 \\
\hline $\mathrm{C}$ & -3.14717100 & 12.30880000 & -5.38798500 \\
\hline $\mathrm{C}$ & -0.83344600 & 11.29291600 & -5.52214300 \\
\hline $\mathrm{C}$ & 1.39548600 & 13.03004800 & -2.30443600 \\
\hline
\end{tabular}

\begin{tabular}{|c|c|c|c|}
\hline $\mathrm{C}$ & 0.40018700 & 13.20256800 & -4.48353100 \\
\hline $\mathrm{C}$ & 0.23448800 & 11.16781600 & -3.28769700 \\
\hline $\mathrm{C}$ & 0.94608300 & 11.69952200 & -2.21800000 \\
\hline $\mathrm{C}$ & 1.11679300 & 13.77711200 & -3.44316600 \\
\hline $\mathrm{C}$ & 1.19744400 & 10.85359800 & -1.05227300 \\
\hline $\mathrm{N}$ & 1.88836700 & 11.31154400 & -0.06117400 \\
\hline $\mathrm{N}$ & 2.06461100 & 10.43810300 & 1.01212900 \\
\hline $\mathrm{C}$ & 2.81460600 & 10.82421700 & 2.07573400 \\
\hline $\mathrm{C}$ & -0.54909200 & 8.46379300 & 13.34516400 \\
\hline $\mathrm{C}$ & -1.27701200 & 11.17627800 & 13.01093600 \\
\hline $\mathrm{C}$ & 0.23251900 & 9.49128100 & 13.89009900 \\
\hline $\mathrm{C}$ & -1.70139200 & 8.82248400 & 12.62159500 \\
\hline $\mathrm{C}$ & -2.05399200 & 10.15801700 & 12.44276600 \\
\hline $\mathrm{C}$ & -0.13304400 & 10.83126900 & 13.73644700 \\
\hline $\mathrm{C}$ & -1.62888300 & 12.63314700 & 12.80705900 \\
\hline $\mathrm{C}$ & -5.51062100 & 13.59865700 & 14.33536800 \\
\hline $\mathrm{C}$ & -3.19799000 & 13.04386100 & 14.69768800 \\
\hline $\mathrm{C}$ & -4.03734000 & 13.14273800 & 12.49058900 \\
\hline $\mathrm{C}$ & -5.31283700 & 13.46377200 & 12.95545400 \\
\hline $\mathrm{C}$ & -4.44208600 & 13.36996100 & 15.20630700 \\
\hline $\mathrm{C}$ & -6.38847700 & 13.66999000 & 11.97807800 \\
\hline $\mathrm{N}$ & -6.23950100 & 13.14701200 & 10.80663500 \\
\hline $\mathrm{N}$ & -7.18747300 & 13.46273100 & 9.82917000 \\
\hline $\mathrm{C}$ & -7.04975000 & 12.88817900 & 8.61334400 \\
\hline $\mathrm{C}$ & -6.90998400 & 12.15879600 & -3.09078900 \\
\hline $\mathrm{C}$ & -7.63136100 & 14.22404700 & -1.29931600 \\
\hline $\mathrm{C}$ & -8.25597400 & 12.53184200 & -2.92062000 \\
\hline $\mathrm{C}$ & -5.94229600 & 12.80704300 & -2.31210700 \\
\hline $\mathrm{C}$ & -6.29711200 & 13.82860400 & -1.42676800 \\
\hline $\mathrm{C}$ & -8.61256100 & 13.55480800 & -2.04493900 \\
\hline $\mathrm{C}$ & -7.98585900 & 15.41724800 & -0.44436800 \\
\hline $\mathrm{C}$ & -10.10894100 & 14.58474000 & 3.17045500 \\
\hline $\mathrm{C}$ & -10.04363200 & 15.50442900 & 0.95720100 \\
\hline $\mathrm{C}$ & -8.15778400 & 14.33497200 & 1.78583900 \\
\hline $\mathrm{C}$ & -8.79973900 & 14.09904000 & 2.99630600 \\
\hline $\mathrm{C}$ & -10.73399900 & 15.27101000 & 2.13635200 \\
\hline $\mathrm{C}$ & -8.07853200 & 13.43468400 & 4.08799900 \\
\hline $\mathrm{N}$ & -8.47934400 & 13.66893200 & 5.29529200 \\
\hline $\mathrm{N}$ & -7.74717600 & 13.05921600 & 6.32742600 \\
\hline $\mathrm{C}$ & -7.97427500 & 13.52024000 & 7.58458100 \\
\hline $\mathrm{N}$ & -8.81476900 & 5.57918000 & -1.03933100 \\
\hline $\mathrm{O}$ & -7.43861700 & 6.24643300 & 5.23855900 \\
\hline $\mathrm{O}$ & -9.37548800 & 3.71404100 & 6.78843400 \\
\hline $\mathrm{N}$ & -5.73394200 & 3.20152700 & 13.04630900 \\
\hline $\mathrm{N}$ & -3.01175000 & 12.95699500 & 13.34935900 \\
\hline $\mathrm{N}$ & -8.77284000 & 15.04842600 & 0.80668100 \\
\hline $\mathrm{N}$ & -0.02262000 & 11.91517500 & -4.38981800 \\
\hline $\mathrm{N}$ & 4.28573200 & 6.89917000 & 8.70446300 \\
\hline $\mathrm{O}$ & 3.41176900 & 11.90414800 & 2.21623300 \\
\hline $\mathrm{O}$ & 2.22359700 & 8.65704200 & 3.00932000 \\
\hline $\mathrm{O}$ & -8.79387900 & 14.39498400 & 7.92028800 \\
\hline $\mathrm{O}$ & -6.25423600 & 11.96394100 & 8.33897500 \\
\hline $\mathrm{H}$ & -7.26113300 & 8.67378600 & -5.35482500 \\
\hline $\mathrm{H}$ & -6.56775800 & 10.30518200 & -1.43751400 \\
\hline $\mathrm{H}$ & -6.94135800 & 8.08290100 & -0.44897700 \\
\hline $\mathrm{H}$ & -7.62766600 & 6.45638300 & -4.36539000 \\
\hline $\mathrm{H}$ & -6.71255300 & 5.54198300 & -0.97770700 \\
\hline $\mathrm{H}$ & -7.43130300 & 4.96087600 & -2.50198300 \\
\hline $\mathrm{H}$ & -12.20044700 & 4.96860700 & 0.66149000 \\
\hline $\mathrm{H}$ & -9.82024700 & 5.62289200 & -2.86923300 \\
\hline $\mathrm{H}$ & -7.93555000 & 5.51651200 & 0.85171600 \\
\hline $\mathrm{H}$ & -12.05850800 & 5.26234900 & -1.82203200 \\
\hline $\mathrm{H}$ & -11.01551100 & 4.53442000 & 2.78485200 \\
\hline $\mathrm{H}$ & -9.93275300 & 4.28775500 & 4.78225100 \\
\hline $\mathrm{H}$ & -2.33337100 & 7.33563100 & 15.05121200 \\
\hline $\mathrm{H}$ & -0.76342300 & 4.61664100 & 12.11998700 \\
\hline $\mathrm{H}$ & -2.71314000 & 3.14277000 & 12.44215600 \\
\hline $\mathrm{H}$ & -4.28963600 & 5.88856300 & 15.35245000 \\
\hline $\mathrm{H}$ & -5.43590000 & 3.92081800 & 15.00373500 \\
\hline $\mathrm{H}$ & -4.44559900 & 2.51398100 & 14.54915300 \\
\hline $\mathrm{H}$ & -8.20014600 & 2.37374800 & 10.23081400 \\
\hline $\mathrm{H}$ & -6.26318900 & 1.39844800 & 13.94868800 \\
\hline $\mathrm{H}$ & -5.28248900 & 4.97222200 & 12.04033100 \\
\hline $\mathrm{H}$ & -7.90521800 & 0.80319300 & 12.16649900 \\
\hline $\mathrm{H}$ & -6.33083800 & 5.61915800 & 9.80715300 \\
\hline $\mathrm{H}$ & -7.15040000 & 5.94472800 & 7.61619600 \\
\hline $\mathrm{H}$ & -7.18023300 & 12.28432100 & -5.77420200 \\
\hline $\mathrm{H}$ & -8.39285000 & 11.07866800 & -5.28670800 \\
\hline $\mathrm{H}$ & -6.95340100 & 10.57990700 & -6.20045300 \\
\hline $\mathrm{H}$ & 1.59439700 & 7.23983900 & 14.83771800 \\
\hline
\end{tabular}




\begin{tabular}{|c|c|c|c|}
\hline $\mathrm{H}$ & 0.74785500 & 5.69050200 & 15.02936900 \\
\hline $\mathrm{H}$ & 0.01623100 & 7.17400300 & 15.66118100 \\
\hline $\mathrm{H}$ & 2.07203800 & 5.28134800 & 13.41999900 \\
\hline $\mathrm{H}$ & -0.30129700 & 7.78668300 & 10.85212500 \\
\hline $\mathrm{H}$ & 1.16236700 & 7.22957200 & 8.96082200 \\
\hline $\mathrm{H}$ & 3.53937200 & 4.74352300 & 11.53424800 \\
\hline $\mathrm{H}$ & 2.88042700 & 5.47764900 & 8.04025600 \\
\hline $\mathrm{H}$ & 4.08633600 & 4.85406700 & 9.19888700 \\
\hline $\mathrm{H}$ & 6.38921800 & 10.06378900 & 8.16411100 \\
\hline $\mathrm{H}$ & 5.33114000 & 6.59372900 & 10.48724400 \\
\hline $\mathrm{H}$ & 3.31364700 & 7.32500400 & 6.90888200 \\
\hline $\mathrm{H}$ & 6.74693400 & 8.62788100 & 10.18362800 \\
\hline $\mathrm{H}$ & 5.15860100 & 10.56114200 & 6.13368100 \\
\hline $\mathrm{H}$ & 4.09591700 & 11.00838200 & 4.14984600 \\
\hline $\mathrm{H}$ & -5.13128500 & 13.09549300 & -5.22784400 \\
\hline $\mathrm{H}$ & -4.55734400 & 9.09916100 & -3.75470600 \\
\hline $\mathrm{H}$ & -2.18348000 & 9.20666000 & -4.39300800 \\
\hline $\mathrm{H}$ & -2.75380400 & 13.20820700 & -5.84918200 \\
\hline $\mathrm{H}$ & -0.66726700 & 11.92166100 & -6.39662500 \\
\hline $\mathrm{H}$ & -0.40896000 & 10.30266200 & -5.69313500 \\
\hline $\mathrm{H}$ & 1.95293000 & 13.45672600 & -1.48157700 \\
\hline $\mathrm{H}$ & 0.15881200 & 13.73759700 & -5.39016800 \\
\hline $\mathrm{H}$ & -0.13066300 & 10.15045900 & -3.28484100 \\
\hline $\mathrm{H}$ & 1.44873400 & 14.80105800 & -3.53675400 \\
\hline $\mathrm{H}$ & 0.77928200 & 9.84831600 & -1.07552200 \\
\hline $\mathrm{H}$ & 1.63473900 & 9.50544000 & 1.02455900 \\
\hline $\mathrm{H}$ & 1.13146200 & 9.25947600 & 14.44558500 \\
\hline $\mathrm{H}$ & -2.32970800 & 8.05003500 & 12.19715800 \\
\hline $\mathrm{H}$ & -2.93971500 & 10.40249100 & 11.86544400 \\
\hline $\mathrm{H}$ & 0.47935500 & 11.61024600 & 14.17723900 \\
\hline $\mathrm{H}$ & -1.64957600 & 12.89986800 & 11.74883100 \\
\hline $\mathrm{H}$ & -0.92715800 & 13.28450100 & 13.32931100 \\
\hline $\mathrm{H}$ & -6.48591600 & 13.86807300 & 14.72118400 \\
\hline $\mathrm{H}$ & -2.33526000 & 12.86504600 & 15.32276400 \\
\hline $\mathrm{H}$ & -3.82879400 & 13.06510600 & 11.43434000 \\
\hline $\mathrm{H}$ & -4.56852000 & 13.45124100 & 16.27627000 \\
\hline $\mathrm{H}$ & -7.24074000 & 14.27656500 & 12.27862000 \\
\hline $\mathrm{H}$ & -7.89939300 & 14.19071400 & 9.96347000 \\
\hline $\mathrm{H}$ & -9.03296100 & 12.03133600 & -3.48462700 \\
\hline $\mathrm{H}$ & -4.90150400 & 12.52989700 & -2.40789800 \\
\hline $\mathrm{H}$ & -5.52821600 & 14.33479100 & -0.85328300 \\
\hline $\mathrm{H}$ & -9.65330900 & 13.84638900 & -1.95149100 \\
\hline $\mathrm{H}$ & -8.61129400 & 16.12462800 & -0.99058200 \\
\hline $\mathrm{H}$ & -7.08698300 & 15.92809500 & -0.09501700 \\
\hline $\mathrm{H}$ & -10.61544700 & 14.41403900 & 4.11060900 \\
\hline $\mathrm{H}$ & -10.47004800 & 16.05813400 & 0.13456800 \\
\hline $\mathrm{H}$ & -7.14874400 & 13.99846600 & 1.59140800 \\
\hline $\mathrm{H}$ & -11.74400300 & 15.64147400 & 2.23655200 \\
\hline $\mathrm{H}$ & -7.22657000 & 12.80530400 & 3.84504100 \\
\hline $\mathrm{H}$ & -7.05078000 & 12.31770300 & 6.09495400 \\
\hline $\mathrm{C}$ & -4.33846200 & 9.90237500 & 1.58991100 \\
\hline $\mathrm{C}$ & -4.55885000 & 8.61586300 & 1.08787700 \\
\hline $\mathrm{C}$ & -5.35469800 & 7.71599400 & 1.80524100 \\
\hline $\mathrm{C}$ & -5.92025900 & 8.08793700 & 3.02674600 \\
\hline $\mathrm{C}$ & -5.68511900 & 9.37373400 & 3.53859300 \\
\hline $\mathrm{C}$ & -4.90443500 & 10.27933900 & 2.80593900 \\
\hline $\mathrm{C}$ & -6.21659800 & 9.86065200 & 4.85130000 \\
\hline $\mathrm{N}$ & -6.67917000 & 8.93548100 & 5.71676300 \\
\hline $\mathrm{O}$ & -6.18810800 & 11.10469000 & 5.11918300 \\
\hline $\mathrm{C}$ & -7.17653800 & 9.32029800 & 7.03668800 \\
\hline $\mathrm{C}$ & -6.53399700 & 8.53121600 & 8.16982100 \\
\hline $\mathrm{N}$ & -6.28878500 & 9.30420800 & 9.26186200 \\
\hline $\mathrm{O}$ & -6.32788800 & 7.29421200 & 8.14331000 \\
\hline $\mathrm{N}$ & -6.13298300 & 8.67410500 & 10.50861900 \\
\hline $\mathrm{C}$ & -5.91012700 & 9.45199600 & 11.51808500 \\
\hline $\mathrm{C}$ & -5.92138800 & 8.88891400 & 12.87262500 \\
\hline $\mathrm{C}$ & -5.42039000 & 9.61937500 & 13.95981400 \\
\hline $\mathrm{C}$ & -5.48678800 & 9.05795600 & 15.23365000 \\
\hline $\mathrm{N}$ & -6.03385500 & 7.84606000 & 15.49045000 \\
\hline $\mathrm{C}$ & -6.52369700 & 7.15318400 & 14.42903900 \\
\hline $\mathrm{C}$ & -6.48220100 & 7.62436800 & 13.12461700 \\
\hline $\mathrm{H}$ & -3.72673700 & 10.60533900 & 1.03712700 \\
\hline $\mathrm{H}$ & -4.12132900 & 8.31776300 & 0.14250600 \\
\hline $\mathrm{H}$ & -5.53724100 & 6.72197000 & 1.41236500 \\
\hline $\mathrm{H}$ & -6.54591200 & 7.37811900 & 3.55365300 \\
\hline $\mathrm{H}$ & -4.73887400 & 11.26926700 & 3.21026600 \\
\hline $\mathrm{H}$ & -6.74306300 & 7.94018800 & 5.48547800 \\
\hline $\mathrm{H}$ & -8.25737100 & 9.13632300 & 7.10805700 \\
\hline $\mathrm{H}$ & -6.99488000 & 10.37884300 & 7.17853900 \\
\hline $\mathrm{H}$ & -6.26625700 & 10.33730200 & 9.18193300 \\
\hline
\end{tabular}

$\begin{array}{rrrr}\mathrm{H} & -5.76575300 & 10.52880500 & 11.42151700 \\ \mathrm{H} & -4.99014300 & 10.60296500 & 13.81938100 \\ \mathrm{H} & -5.09614200 & 9.60531200 & 16.08349400 \\ \mathrm{H} & -6.96572000 & 6.18679100 & 14.64176300 \\ \mathrm{H} & -6.89518200 & 7.04046300 & 12.31334500\end{array}$

\section{Complex C2•7b-1}

E -5753.993883 a.u. (zero imaginary freq.)

\begin{tabular}{|c|c|c|c|}
\hline $\mathrm{C}$ & -16.10909000 & 0.28441600 & -9.58685200 \\
\hline $\mathrm{C}$ & -17.40634600 & -1.68282200 & -8.02270400 \\
\hline $\mathrm{C}$ & -16.64014400 & -0.87033500 & -10.17752700 \\
\hline $\mathrm{C}$ & -16.22032700 & 0.42530700 & -8.19149900 \\
\hline $\mathrm{C}$ & -16.86201300 & -0.54110800 & -7.41842900 \\
\hline $\mathrm{C}$ & -17.28136100 & -1.84266800 & -9.40585500 \\
\hline C & -18.14884200 & -2.71502700 & -7.20384500 \\
\hline $\mathrm{C}$ & -15.54655600 & -4.65187800 & -4.39770500 \\
\hline $\mathrm{C}$ & -17.50381100 & -3.33275700 & -4.87369200 \\
\hline$C$ & -16.13729700 & -4.07043700 & -6.66073300 \\
\hline $\mathrm{C}$ & -15.28623200 & -4.70435500 & -5.77830000 \\
\hline $\mathrm{C}$ & -16.68070600 & -3.94839500 & -3.95582300 \\
\hline $\mathrm{C}$ & -14.64181600 & -5.31056700 & -3.46036500 \\
\hline $\mathrm{N}$ & -14.91018800 & -5.27790800 & -2.19433600 \\
\hline $\mathrm{N}$ & -14.00277700 & -5.89982900 & -1.34941200 \\
\hline $\mathrm{C}$ & -14.23962600 & -5.89696300 & -0.00840200 \\
\hline $\mathrm{C}$ & -7.67142400 & -4.10354800 & 9.67485600 \\
\hline $\mathrm{C}$ & -8.39953700 & -6.69801800 & 8.81441600 \\
\hline $\mathrm{C}$ & -8.44694600 & -4.94423200 & 10.49002700 \\
\hline $\mathrm{C}$ & -7.26195000 & -4.59154800 & 8.42448800 \\
\hline $\mathrm{C}$ & -7.61666700 & -5.87244600 & 8.00045100 \\
\hline $\mathrm{C}$ & -8.80874000 & -6.22398300 & 10.06655200 \\
\hline $\mathrm{C}$ & -8.83700000 & -8.06351800 & 8.32617900 \\
\hline $\mathrm{C}$ & -11.72616500 & -7.31896900 & 5.25177900 \\
\hline $\mathrm{C}$ & -9.53558900 & -8.05599500 & 5.92720300 \\
\hline $\mathrm{C}$ & -11.13271200 & -7.48790700 & 7.58074900 \\
\hline $\mathrm{C}$ & -12.06883500 & -7.19826200 & 6.60993300 \\
\hline $\mathrm{C}$ & -10.43726000 & -7.77431400 & 4.92254800 \\
\hline $\mathrm{C}$ & -12.69525100 & -6.92967100 & 4.23360400 \\
\hline $\mathrm{N}$ & -12.38392000 & -7.00872100 & 2.97916700 \\
\hline $\mathrm{N}$ & -13.34252700 & -6.54280900 & 2.08935200 \\
\hline $\mathrm{C}$ & -13.09634900 & -6.56721500 & 0.75046600 \\
\hline $\mathrm{C}$ & -15.36945300 & 1.34354300 & -10.43742400 \\
\hline $\mathrm{C}$ & -15.99684000 & 1.42488100 & -11.86549400 \\
\hline $\mathrm{C}$ & -7.35814900 & -2.65833300 & 10.13269200 \\
\hline $\mathrm{C}$ & -6.87337100 & -2.67337400 & 11.61661100 \\
\hline $\mathrm{C}$ & -6.23421400 & -2.04656100 & 9.25822800 \\
\hline $\mathrm{C}$ & -4.14455500 & -0.95396500 & 7.69405600 \\
\hline $\mathrm{C}$ & -4.93172100 & -2.56932000 & 9.32554800 \\
\hline $\mathrm{C}$ & -6.46934600 & -0.97445400 & 8.38542100 \\
\hline $\mathrm{C}$ & -5.43874400 & -0.42899900 & 7.61912300 \\
\hline $\mathrm{C}$ & -3.90111100 & -2.03480700 & 8.55072400 \\
\hline $\mathrm{C}$ & -3.01534500 & -0.34855300 & 6.88466900 \\
\hline $\mathrm{C}$ & -3.85577100 & -0.86053200 & 2.71345400 \\
\hline $\mathrm{C}$ & -3.78643100 & 0.52674400 & 4.67910800 \\
\hline $\mathrm{C}$ & -3.02578900 & -1.71173600 & 4.80854800 \\
\hline $\mathrm{C}$ & -3.29768400 & -1.90600500 & 3.46884500 \\
\hline $\mathrm{C}$ & -4.09195000 & 0.37374600 & 3.34318900 \\
\hline $\mathrm{C}$ & -4.19228900 & -1.08190500 & 1.30922100 \\
\hline $\mathrm{N}$ & -4.94649400 & -0.22724600 & 0.69489900 \\
\hline $\mathrm{N}$ & -5.20055500 & -0.49226300 & -0.64754600 \\
\hline $\mathrm{C}$ & -6.01720200 & 0.32346500 & -1.35522900 \\
\hline $\mathrm{C}$ & -13.87263100 & 0.96065600 & -10.55827200 \\
\hline $\mathrm{C}$ & -11.11947500 & 0.33561500 & -10.76705100 \\
\hline $\mathrm{C}$ & -13.06992000 & 1.54611900 & -11.55144100 \\
\hline $\mathrm{C}$ & -13.26756700 & 0.05038200 & -9.67621300 \\
\hline $\mathrm{C}$ & -11.91212600 & -0.26357400 & -9.78059400 \\
\hline $\mathrm{C}$ & -11.71021900 & 1.24431800 & -11.65067500 \\
\hline $\mathrm{C}$ & -9.63903100 & 0.02695800 & -10.86446300 \\
\hline $\mathrm{C}$ & -8.00952800 & 0.92526700 & -7.01253100 \\
\hline $\mathrm{C}$ & -8.37081200 & -0.62924700 & -8.81431900 \\
\hline $\mathrm{C}$ & -9.06511200 & 1.62175200 & -9.06174800 \\
\hline $\mathrm{C}$ & -8.59407000 & 1.93012200 & -7.80388400 \\
\hline $\mathrm{C}$ & -7.87996500 & -0.36530400 & -7.55274500 \\
\hline $\mathrm{C}$ & -7.64210600 & 1.23065000 & -5.63459400 \\
\hline $\mathrm{N}$ & -7.04652900 & 0.33239500 & -4.91414900 \\
\hline $\mathrm{N}$ & -6.86907800 & 0.67300100 & -3.56583300 \\
\hline $\mathrm{C}$ & -6.07030300 & -0.12271400 & -2.81493500 \\
\hline $\mathrm{C}$ & -8.63781500 & -1.79534800 & 10.00318200 \\
\hline $\mathrm{C}$ & -10.87555400 & -0.10773800 & 9.61821900 \\
\hline $\mathrm{C}$ & -8.73471100 & -0.56275200 & 10.66823300 \\
\hline $\mathrm{C}$ & -9.69134500 & -2.17427200 & 9.15635800 \\
\hline
\end{tabular}




\begin{tabular}{|c|c|c|c|}
\hline $\mathrm{C}$ & -10.80041200 & -1.34873600 & 8.97301100 \\
\hline $\mathrm{C}$ & -9.83394800 & 0.27557500 & 10.47177100 \\
\hline $\mathrm{C}$ & -12.03913300 & 0.81763000 & 9.36380000 \\
\hline $\mathrm{C}$ & -12.02828300 & 2.39470000 & 5.35896000 \\
\hline $\mathrm{C}$ & -13.18926700 & 1.80290900 & 7.38449200 \\
\hline $\mathrm{C}$ & -10.85031800 & 1.47520800 & 7.25190400 \\
\hline $\mathrm{C}$ & -10.83325300 & 1.97383100 & 5.96391700 \\
\hline $\mathrm{C}$ & -13.21881900 & 2.31520000 & 6.10639900 \\
\hline $\mathrm{C}$ & -11.99839100 & 2.86880600 & 3.97958800 \\
\hline $\mathrm{N}$ & -13.07022200 & 3.37749100 & 3.45459200 \\
\hline $\mathrm{N}$ & -12.95706900 & 3.78380800 & 2.12115600 \\
\hline $\mathrm{C}$ & -14.05322700 & 4.31198500 & 1.52321600 \\
\hline $\mathrm{C}$ & -15.53329100 & 2.74069400 & -9.78593400 \\
\hline $\mathrm{C}$ & -15.92915500 & 5.32107500 & -8.69643700 \\
\hline $\mathrm{C}$ & -16.82856200 & 3.25799800 & -9.60093100 \\
\hline $\mathrm{C}$ & -14.44445400 & 3.53555200 & -9.40592900 \\
\hline $\mathrm{C}$ & -14.63856900 & 4.81386800 & -8.87410600 \\
\hline $\mathrm{C}$ & -17.02575400 & 4.52724900 & -9.06102900 \\
\hline $\mathrm{C}$ & -16.14212800 & 6.69044300 & -8.08519800 \\
\hline $\mathrm{C}$ & -16.05148400 & 6.11931900 & -3.83958000 \\
\hline $\mathrm{C}$ & -14.92959100 & 6.56794900 & -5.92066200 \\
\hline $\mathrm{C}$ & -17.29126600 & 6.40667100 & -5.88521900 \\
\hline $\mathrm{C}$ & -17.27735500 & 6.18542800 & -4.52017600 \\
\hline $\mathrm{C}$ & -14.86628400 & 6.33922600 & -4.56512500 \\
\hline $\mathrm{C}$ & -16.01924200 & 5.78319900 & -2.41756700 \\
\hline $\mathrm{N}$ & -14.88602600 & 5.46950200 & -1.87658300 \\
\hline $\mathrm{N}$ & -14.91215400 & 5.13157300 & -0.52938000 \\
\hline $\mathrm{C}$ & -13.78481500 & 4.66051400 & 0.06035700 \\
\hline $\mathrm{N}$ & -17.23055600 & -3.39930500 & -6.20678500 \\
\hline $\mathrm{O}$ & -15.22679700 & -5.41474100 & 0.56715000 \\
\hline $\mathrm{O}$ & -12.10032800 & -7.03964100 & 0.18298300 \\
\hline $\mathrm{N}$ & -9.88300800 & -7.90073700 & 7.23568100 \\
\hline $\mathrm{N}$ & -12.01580400 & 1.38316500 & 7.94218700 \\
\hline $\mathrm{N}$ & -16.12930100 & 6.58457300 & -6.56767000 \\
\hline $\mathrm{N}$ & -8.96472800 & 0.35337900 & -9.54663500 \\
\hline $\mathrm{N}$ & -3.26921500 & -0.50923400 & 5.39670400 \\
\hline $\mathrm{O}$ & -5.41709300 & -1.10704700 & -3.20568400 \\
\hline $\mathrm{O}$ & -6.64887200 & 1.30364600 & -0.91571600 \\
\hline $\mathrm{O}$ & -12.67989600 & 4.50526500 & -0.48950500 \\
\hline $\mathrm{O}$ & -15.17114700 & 4.50226900 & 2.03373600 \\
\hline $\mathrm{H}$ & -16.55970700 & -1.02533200 & -11.24526500 \\
\hline $\mathrm{H}$ & -15.80571500 & 1.30222700 & -7.70923900 \\
\hline $\mathrm{H}$ & -16.94431700 & -0.40629700 & -6.34510200 \\
\hline $\mathrm{H}$ & -17.69422000 & -2.72361000 & -9.88494800 \\
\hline $\mathrm{H}$ & -18.56527600 & -3.49712200 & -7.84119300 \\
\hline $\mathrm{H}$ & -18.94855300 & -2.26383800 & -6.61477000 \\
\hline $\mathrm{H}$ & -18.38725700 & -2.78474700 & -4.58111800 \\
\hline $\mathrm{H}$ & -15.97456400 & -4.07802500 & -7.72866900 \\
\hline $\mathrm{H}$ & -14.42510300 & -5.23531800 & -6.16083700 \\
\hline $\mathrm{H}$ & -16.91052900 & -3.88456000 & -2.90261700 \\
\hline $\mathrm{H}$ & -13.76536400 & -5.80448600 & -3.87617700 \\
\hline $\mathrm{H}$ & -13.14778800 & -6.34442700 & -1.70505000 \\
\hline $\mathrm{H}$ & -8.79375100 & -4.59695800 & 11.45519700 \\
\hline $\mathrm{H}$ & -6.67103900 & -3.96495500 & 7.77083400 \\
\hline $\mathrm{H}$ & -7.29358800 & -6.22257700 & 7.02555900 \\
\hline $\mathrm{H}$ & -9.41443400 & -6.85344100 & 10.70932600 \\
\hline $\mathrm{H}$ & -9.29580900 & -8.64628100 & 9.12580900 \\
\hline $\mathrm{H}$ & -8.01457300 & -8.62522400 & 7.88248000 \\
\hline $\mathrm{H}$ & -8.53103300 & -8.39730500 & 5.72455500 \\
\hline $\mathrm{H}$ & -11.34151900 & -7.38604000 & 8.63599900 \\
\hline $\mathrm{H}$ & -13.05417300 & -6.86384900 & 6.90502400 \\
\hline $\mathrm{H}$ & -10.14153400 & -7.88896600 & 3.89007300 \\
\hline $\mathrm{H}$ & -13.65293100 & -6.55041900 & 4.58652300 \\
\hline $\mathrm{H}$ & -14.22050600 & -6.12158500 & 2.41788000 \\
\hline $\mathrm{H}$ & -15.59225400 & 2.28525900 & -12.40365200 \\
\hline $\mathrm{H}$ & -17.08085100 & 1.54501100 & -11.79063000 \\
\hline $\mathrm{H}$ & -15.77740200 & 0.52178300 & -12.44117700 \\
\hline $\mathrm{H}$ & -6.51360800 & -1.68406900 & 11.91030800 \\
\hline $\mathrm{H}$ & -6.05457500 & -3.38741900 & 11.73687200 \\
\hline $\mathrm{H}$ & -7.68956200 & -2.95868300 & 12.28529300 \\
\hline $\mathrm{H}$ & -4.71759800 & -3.40797800 & 9.97686200 \\
\hline $\mathrm{H}$ & -7.46243000 & -0.55572600 & 8.30634400 \\
\hline $\mathrm{H}$ & -5.65098500 & 0.40894200 & 6.96402900 \\
\hline $\mathrm{H}$ & -2.90287600 & -2.45332500 & 8.61818800 \\
\hline $\mathrm{H}$ & -2.06616300 & -0.84094800 & 7.09927000 \\
\hline $\mathrm{H}$ & -2.91866500 & 0.72339100 & 7.06458600 \\
\hline $\mathrm{H}$ & -3.93789400 & 1.45935800 & 5.20232400 \\
\hline $\mathrm{H}$ & -2.61556500 & -2.48973500 & 5.43587400 \\
\hline $\mathrm{H}$ & -3.09001300 & -2.86740100 & 3.01886500 \\
\hline $\mathrm{H}$ & -4.49973000 & 1.20703300 & 2.79032400 \\
\hline
\end{tabular}

\begin{tabular}{|c|c|c|c|}
\hline $\mathrm{H}$ & -3.79393600 & -1.97700700 & 0.83463700 \\
\hline $\mathrm{H}$ & -4.75574400 & -1.27853100 & -1.13967600 \\
\hline $\mathrm{H}$ & -13.49445200 & 2.26070200 & -12.24465100 \\
\hline $\mathrm{H}$ & -13.85931100 & -0.41976200 & -8.90282700 \\
\hline $\mathrm{H}$ & -11.47179100 & -0.97352000 & -9.08826400 \\
\hline $\mathrm{H}$ & -11.10699900 & 1.71964100 & -12.41638300 \\
\hline $\mathrm{H}$ & -9.15946000 & 0.63037800 & -11.63687300 \\
\hline $\mathrm{H}$ & -9.44914700 & -1.03065600 & -11.05417200 \\
\hline $\mathrm{H}$ & -8.32389300 & -1.61204600 & -9.25922200 \\
\hline $\mathrm{H}$ & -9.54689900 & 2.35166100 & -9.69574600 \\
\hline $\mathrm{H}$ & -8.70150700 & 2.93866400 & -7.42869600 \\
\hline $\mathrm{H}$ & -7.43523500 & -1.16041400 & -6.97249300 \\
\hline $\mathrm{H}$ & -7.94030400 & 2.19908700 & -5.24841200 \\
\hline $\mathrm{H}$ & -7.45035700 & 1.43437900 & -3.15797100 \\
\hline $\mathrm{H}$ & -7.93720500 & -0.23152100 & 11.32084500 \\
\hline $\mathrm{H}$ & -9.63171500 & -3.10722300 & 8.61464300 \\
\hline $\mathrm{H}$ & -11.60071900 & -1.66368900 & 8.31198500 \\
\hline $\mathrm{H}$ & -9.87833000 & 1.23397300 & 10.97729300 \\
\hline $\mathrm{H}$ & -12.02014600 & 1.67470200 & 10.03991900 \\
\hline $\mathrm{H}$ & -12.99607000 & 0.30133500 & 9.45580800 \\
\hline $\mathrm{H}$ & -14.07913400 & 1.69930300 & 7.98857200 \\
\hline $\mathrm{H}$ & -9.95689600 & 1.13382600 & 7.75099300 \\
\hline $\mathrm{H}$ & -9.89516600 & 2.02348000 & 5.42897900 \\
\hline $\mathrm{H}$ & -14.15820800 & 2.63201400 & 5.67740300 \\
\hline $\mathrm{H}$ & -11.06916100 & 2.74811500 & 3.43036200 \\
\hline $\mathrm{H}$ & -12.06357700 & 3.63863100 & 1.60349900 \\
\hline $\mathrm{H}$ & -17.69079200 & 2.66201400 & -9.87529300 \\
\hline $\mathrm{H}$ & -13.43715900 & 3.16552400 & -9.53646200 \\
\hline $\mathrm{H}$ & -13.77859300 & 5.41650000 & -8.60193600 \\
\hline $\mathrm{H}$ & -18.03291200 & 4.90757100 & -8.92928000 \\
\hline $\mathrm{H}$ & -17.10842700 & 7.11153900 & -8.36316700 \\
\hline $\mathrm{H}$ & -15.34381400 & 7.38367600 & -8.35221900 \\
\hline $\mathrm{H}$ & -14.04775900 & 6.73231600 & -6.52178900 \\
\hline $\mathrm{H}$ & -18.20743200 & 6.43596900 & -6.45662500 \\
\hline $\mathrm{H}$ & -18.21266200 & 6.03965600 & -3.99722400 \\
\hline $\mathrm{H}$ & -13.90695100 & 6.32170400 & -4.07051200 \\
\hline $\mathrm{H}$ & -16.96476100 & 5.78437000 & -1.87780900 \\
\hline $\mathrm{H}$ & -15.76951400 & 5.19397100 & 0.03464100 \\
\hline $\mathrm{C}$ & -12.06055200 & 1.64259300 & -5.70961000 \\
\hline $\mathrm{C}$ & -13.29169500 & 2.27444700 & -5.50848000 \\
\hline $\mathrm{C}$ & -13.47310700 & 3.09596400 & -4.39237300 \\
\hline $\mathrm{C}$ & -12.43197700 & 3.30109200 & -3.48342500 \\
\hline $\mathrm{C}$ & -11.19616800 & 2.66810900 & -3.68196600 \\
\hline $\mathrm{C}$ & -11.02269100 & 1.83878800 & -4.80089300 \\
\hline $\mathrm{C}$ & -10.01335200 & 2.83003600 & -2.77501100 \\
\hline $\mathrm{N}$ & -10.20875800 & 3.39072000 & -1.56206100 \\
\hline $\mathrm{O}$ & -8.86643500 & 2.44154900 & -3.15366700 \\
\hline $\mathrm{C}$ & -9.09616500 & 3.50184600 & -0.60220400 \\
\hline $\mathrm{C}$ & -9.45992000 & 2.75720700 & 0.67816800 \\
\hline $\mathrm{N}$ & -8.54809400 & 1.83118300 & 1.06420200 \\
\hline $\mathrm{O}$ & -10.51348100 & 2.99240600 & 1.31443400 \\
\hline $\mathrm{N}$ & -8.84295700 & 1.04854800 & 2.19818000 \\
\hline $\mathrm{C}$ & -7.95942300 & 0.16020200 & 2.51825600 \\
\hline $\mathrm{C}$ & -8.21751600 & -0.75192500 & 3.63634200 \\
\hline $\mathrm{C}$ & -9.47471500 & -0.80811600 & 4.26206200 \\
\hline $\mathrm{C}$ & -9.66928900 & -1.70325200 & 5.30459900 \\
\hline $\mathrm{N}$ & -8.70371000 & -2.54442600 & 5.75403500 \\
\hline $\mathrm{C}$ & -7.49484800 & -2.47942400 & 5.14879700 \\
\hline $\mathrm{C}$ & -7.20936100 & -1.61161700 & 4.09669700 \\
\hline $\mathrm{H}$ & -11.91101800 & 1.00557300 & -6.57393600 \\
\hline $\mathrm{H}$ & -14.09982200 & 2.12974700 & -6.21728100 \\
\hline $\mathrm{H}$ & -14.42266200 & 3.58863500 & -4.22672400 \\
\hline $\mathrm{H}$ & -12.60296200 & 3.95189400 & -2.63601800 \\
\hline $\mathrm{H}$ & -10.07484100 & 1.34116700 & -4.94205800 \\
\hline $\mathrm{H}$ & -11.12819000 & 3.69105100 & -1.22708100 \\
\hline $\mathrm{H}$ & -8.92248900 & 4.55215800 & -0.34621700 \\
\hline $\mathrm{H}$ & -8.20873700 & 3.08380600 & -1.06793500 \\
\hline $\mathrm{H}$ & -7.71398200 & 1.61799500 & 0.48500200 \\
\hline $\mathrm{H}$ & -7.01886200 & 0.03734300 & 1.98206200 \\
\hline $\mathrm{H}$ & -10.27770100 & -0.16741000 & 3.92587300 \\
\hline $\mathrm{H}$ & -10.63200400 & -1.75915700 & 5.79891900 \\
\hline $\mathrm{H}$ & -6.72848100 & -3.14970600 & 5.52005600 \\
\hline $\mathrm{H}$ & -6.22548500 & -1.60142200 & 3.64789400 \\
\hline
\end{tabular}

\section{Complex C2•7b -2}

E -5753.993841 a.u. (zero imaginary freq.)

$\begin{array}{llll}\text { C } & -16.20035800 & 0.32175700 & -9.33524200\end{array}$

$\begin{array}{llll}\text { C } & -17.54062800 & -1.58752700 & -7.73534700\end{array}$

$\begin{array}{llll}\text { C } & -16.78095800 & -0.81778100 & -9.90855500\end{array}$ 


\begin{tabular}{|c|c|c|c|}
\hline $\mathrm{C}$ & -16.28481300 & 0.47650600 & -7.93954200 \\
\hline $\mathrm{C}$ & -16.94737600 & -0.46089700 & -7.14883400 \\
\hline $\mathrm{C}$ & -17.44334200 & -1.76137000 & -9.11918900 \\
\hline $\mathrm{C}$ & -18.30318500 & -2.58895500 & -6.89683800 \\
\hline $\mathrm{C}$ & -15.72167900 & -4.58546500 & -4.11255800 \\
\hline $\mathrm{C}$ & -17.66737700 & -3.24474000 & -4.57482600 \\
\hline $\mathrm{C}$ & -16.30424700 & -3.97084800 & -6.36894500 \\
\hline $\mathrm{C}$ & -15.46023400 & -4.62347200 & -5.49337100 \\
\hline $\mathrm{C}$ & -16.85125900 & -3.87917100 & -3.66365500 \\
\hline $\mathrm{C}$ & -14.82097000 & -5.25844000 & -3.18154900 \\
\hline $\mathrm{N}$ & -15.08528100 & -5.22973600 & -1.91453300 \\
\hline $\mathrm{N}$ & -14.17979800 & -5.86246600 & -1.07570600 \\
\hline $\mathrm{C}$ & -14.40954300 & -5.86179200 & 0.26653000 \\
\hline $\mathrm{C}$ & -7.70719800 & -4.12803500 & 9.86549600 \\
\hline $\mathrm{C}$ & -8.49170700 & -6.71320600 & 9.02818000 \\
\hline $\mathrm{C}$ & -8.50934800 & -4.94026200 & 10.68385200 \\
\hline $\mathrm{C}$ & -7.30236400 & -4.63844400 & 8.62286500 \\
\hline $\mathrm{C}$ & -7.68559100 & -5.91503900 & 8.20977300 \\
\hline $\mathrm{C}$ & -8.89796200 & -6.21571400 & 10.27231200 \\
\hline $\mathrm{C}$ & -8.95478200 & -8.07500900 & 8.55423800 \\
\hline $\mathrm{C}$ & -11.86611000 & -7.31058600 & 5.50490100 \\
\hline $\mathrm{C}$ & -9.67310400 & -8.05764800 & 6.16123100 \\
\hline $\mathrm{C}$ & -11.25516200 & -7.48906600 & 7.82859800 \\
\hline $\mathrm{C}$ & -12.19771300 & -7.19296300 & 6.86594100 \\
\hline $\mathrm{C}$ & -10.58147800 & -7.76986000 & 5.16442100 \\
\hline $\mathrm{C}$ & -12.84150400 & -6.91433100 & 4.49544100 \\
\hline $\mathrm{N}$ & -12.54063200 & -6.99265100 & 3.23848200 \\
\hline $\mathrm{N}$ & -13.50357300 & -6.52006300 & 2.35693500 \\
\hline $\mathrm{C}$ & -13.26653800 & -6.54244200 & 1.01640400 \\
\hline $\mathrm{C}$ & -15.43660600 & 1.34912900 & -10.20304800 \\
\hline $\mathrm{C}$ & -16.07252000 & 1.43539900 & -11.62672400 \\
\hline $\mathrm{C}$ & -7.35707900 & -2.68983200 & 10.31915600 \\
\hline $\mathrm{C}$ & -6.84667400 & -2.72130500 & 11.79430500 \\
\hline $\mathrm{C}$ & -6.23256100 & -2.09292500 & 9.43549400 \\
\hline $\mathrm{C}$ & -4.13351300 & -1.01380500 & 7.87498700 \\
\hline $\mathrm{C}$ & -4.94750800 & -2.66171300 & 9.45943000 \\
\hline $\mathrm{C}$ & -6.44451800 & -0.97864200 & 8.61080800 \\
\hline $\mathrm{C}$ & -5.40910700 & -0.44147800 & 7.84515000 \\
\hline $\mathrm{C}$ & -3.91261800 & -2.13416900 & 8.68599500 \\
\hline $\mathrm{C}$ & -3.00304300 & -0.42071300 & 7.05930600 \\
\hline $\mathrm{C}$ & -3.87722500 & -0.95327700 & 2.89740200 \\
\hline $\mathrm{C}$ & -3.79019000 & 0.44481800 & 4.85476100 \\
\hline $\mathrm{C}$ & -3.03094700 & -1.79394000 & 4.98994900 \\
\hline $\mathrm{C}$ & -3.31496700 & -1.99555000 & 3.65384800 \\
\hline $\mathrm{C}$ & -4.10715900 & 0.28469000 & 3.52244000 \\
\hline $\mathrm{C}$ & -4.22203900 & -1.18098900 & 1.49635800 \\
\hline $\mathrm{N}$ & -4.97194500 & -0.32473100 & 0.87909600 \\
\hline $\mathrm{N}$ & -5.23224700 & -0.59696500 & -0.46072400 \\
\hline $\mathrm{C}$ & -6.04277700 & 0.22206900 & -1.17146700 \\
\hline $\mathrm{C}$ & -13.95250900 & 0.92152400 & -10.33070500 \\
\hline $\mathrm{C}$ & -11.21806000 & 0.22008700 & -10.54583600 \\
\hline $\mathrm{C}$ & -13.13818400 & 1.47829700 & -11.33090000 \\
\hline $\mathrm{C}$ & -13.36932700 & -0.00059600 & -9.44635600 \\
\hline $\mathrm{C}$ & -12.02356500 & -0.35229400 & -9.55394600 \\
\hline $\mathrm{C}$ & -11.78746700 & 1.13907500 & -11.43311800 \\
\hline $\mathrm{C}$ & -9.74696000 & -0.12886400 & -10.64445100 \\
\hline $\mathrm{C}$ & -8.06073100 & 0.79820000 & -6.82241000 \\
\hline $\mathrm{C}$ & -8.43893200 & -0.76628400 & -8.61216800 \\
\hline $\mathrm{C}$ & -9.15241800 & 1.47875300 & -8.85807300 \\
\hline $\mathrm{C}$ & -8.66165800 & 1.79620300 & -7.61004700 \\
\hline $\mathrm{C}$ & -7.93036700 & -0.49371100 & -7.35939100 \\
\hline $\mathrm{C}$ & -7.67903700 & 1.11274800 & -5.45048500 \\
\hline $\mathrm{N}$ & -7.08601800 & 0.21591400 & -4.72617800 \\
\hline $\mathrm{N}$ & -6.89736500 & 0.56661100 & -3.38200600 \\
\hline $\mathrm{C}$ & -6.10534500 & -0.23298400 & -2.62790700 \\
\hline $\mathrm{C}$ & -8.62364400 & -1.80746600 & 10.20137500 \\
\hline $\mathrm{C}$ & -10.82480400 & -0.07272800 & 9.81786300 \\
\hline $\mathrm{C}$ & -8.76049100 & -0.63826100 & 10.96347100 \\
\hline $\mathrm{C}$ & -9.61710400 & -2.09969900 & 9.25239800 \\
\hline $\mathrm{C}$ & -10.71075900 & -1.25473100 & 9.07335500 \\
\hline $\mathrm{C}$ & -9.84046500 & 0.22626100 & 10.76597800 \\
\hline $\mathrm{C}$ & -11.96900700 & 0.88104500 & 9.57371500 \\
\hline $\mathrm{C}$ & -11.97299700 & 2.43206600 & 5.56071800 \\
\hline $\mathrm{C}$ & -13.12473800 & 1.86140200 & 7.59703300 \\
\hline $\mathrm{C}$ & -10.78660000 & 1.52939900 & 7.45624100 \\
\hline $\mathrm{C}$ & -10.77538700 & 2.01413400 & 6.16310300 \\
\hline $\mathrm{C}$ & -13.15942400 & 2.36408000 & 6.31535600 \\
\hline $\mathrm{C}$ & -11.95012200 & 2.89089300 & 4.17646600 \\
\hline $\mathrm{N}$ & -13.01960900 & 3.40971800 & 3.65630300 \\
\hline $\mathrm{N}$ & -12.91152100 & 3.80154700 & 2.31830700 \\
\hline
\end{tabular}

\begin{tabular}{|c|c|c|c|}
\hline $\mathrm{C}$ & -14.00057300 & 4.34947100 & 1.72476400 \\
\hline $\mathrm{C}$ & -15.55266200 & 2.75698400 & -9.56342400 \\
\hline $\mathrm{C}$ & -15.85963200 & 5.35801400 & -8.49496900 \\
\hline $\mathrm{C}$ & -16.82997300 & 3.31397000 & -9.36836200 \\
\hline 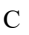 & -14.43670300 & 3.52271000 & -9.20331200 \\
\hline $\mathrm{C}$ & -14.58686100 & 4.81122800 & -8.68198400 \\
\hline 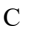 & -16.98327500 & 4.59356400 & -8.83857400 \\
\hline $\mathrm{C}$ & -16.02462200 & 6.73913000 & -7.89589600 \\
\hline$C$ & -15.94871100 & 6.19329200 & -3.64746400 \\
\hline $\mathrm{C}$ & -14.81231700 & 6.57234400 & -5.73468300 \\
\hline $\mathrm{C}$ & -17.17882000 & 6.52785700 & -5.69190100 \\
\hline 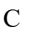 & -17.17173700 & 6.31343000 & -4.32575000 \\
\hline $\mathrm{C}$ & -14.75612100 & 6.34999700 & -4.37777200 \\
\hline $\mathrm{C}$ & -15.92983500 & 5.86545800 & -2.22311000 \\
\hline $\mathrm{N}$ & -14.80929900 & 5.51463500 & -1.67903000 \\
\hline $\mathrm{N}$ & -14.84622300 & 5.18417200 & -0.33033900 \\
\hline $\mathrm{C}$ & -13.73154400 & 4.68321200 & 0.25885800 \\
\hline $\mathrm{N}$ & -17.39208300 & -3.29580800 & -5.90822200 \\
\hline $\mathrm{O}$ & -15.38989900 & -5.37410000 & 0.84903800 \\
\hline $\mathrm{O}$ & -12.27721200 & -7.01977500 & 27800 \\
\hline $\mathrm{N}$ & -10. & 6900 & 89800 \\
\hline $\mathrm{N}$ & -11.94954800 & 1.44379200 & 8.15265400 \\
\hline $\mathrm{N}$ & -16.01174800 & 6.64532200 & -6.37829600 \\
\hline $\mathrm{N}$ & -9.05387900 & 0.20838700 & -9.33791800 \\
\hline $\mathrm{N}$ & -3.26 & -0.58 & 6200 \\
\hline $\mathrm{O}$ & -5.46486300 & -1.227 & -3.01 \\
\hline $\mathrm{O}$ & -6.66329300 & 1.211 & -0.73 \\
\hline $\mathrm{O}$ & 386300 & 64500 & 64000 \\
\hline $\mathrm{O}$ & -15.11029100 & 4.56818100 & 2.24132200 \\
\hline $\mathrm{H}$ & -16.72288700 & -0.98379000 & -10.97611400 \\
\hline $\mathrm{H}$ & -15.83154500 & 3200 & 97100 \\
\hline $\mathrm{H}$ & 7600 & -0.31414900 & -6.07575000 \\
\hline $\mathrm{H}$ & 5700 & 3000 & 1100 \\
\hline $\mathrm{H}$ & -18.75458700 & 500 & 7600 \\
\hline $\mathrm{H}$ & 860600 & 8600 & 81800 \\
\hline $\mathrm{H}$ & -18.54604800 & -2.69244700 & -4.27632000 \\
\hline $\mathrm{H}$ & -16.13909900 & -3.96600200 & -7.43652200 \\
\hline $\mathrm{H}$ & -14.60285100 & -5.15647300 & -5.88141700 \\
\hline $\mathrm{H}$ & 6500 & -3.8 & 04100 \\
\hline $\mathrm{H}$ & 54700 & 9400 & 4500 \\
\hline $\mathrm{H}$ & -13.32980800 & 8100 & 3500 \\
\hline $\mathrm{H}$ & -8.85155200 & -4.573 & 11.64364600 \\
\hline $\mathrm{H}$ & -6.69067300 & -4.03338300 & 7.96807200 \\
\hline $\mathrm{H}$ & -7.36496900 & -6.28419800 & 7.24102400 \\
\hline $\mathrm{H}$ & -9.52112700 & -6.82436000 & 10.91839400 \\
\hline $\mathrm{H}$ & -9.41450000 & -8.645 & 8500 \\
\hline $\mathrm{H}$ & -8.14 & 6000 & 7800 \\
\hline $\mathrm{H}$ & -8.67 & -8.40 & 3200 \\
\hline $\mathrm{H}$ & -11.45623200 & -7.39015800 & 8.88561700 \\
\hline $\mathrm{H}$ & -13.17934500 & -6.85576800 & 7.17006200 \\
\hline $\mathrm{H}$ & -10.29419100 & -7.88272600 & 4.12936700 \\
\hline $\mathrm{H}$ & -13 & -6.5 & 1100 \\
\hline $\mathrm{H}$ & 39900 & 23100 & 01300 \\
\hline $\mathrm{H}$ & 97500 & 2.27706300 & -12.17728000 \\
\hline $\mathrm{H}$ & -17.15174600 & 1.58954800 & -11.54479200 \\
\hline $\mathrm{H}$ & -15.88585600 & 0.52000200 & -12.19444700 \\
\hline $\mathrm{H}$ & -6.44946500 & -1.74477200 & 12.08346100 \\
\hline $\mathrm{H}$ & -6.04720000 & -3.45984200 & 11.89587200 \\
\hline $\mathrm{H}$ & & -2.98557300 & 12.47766000 \\
\hline $\mathrm{H}$ & -4.75174600 & -3.52998400 & 10.07 \\
\hline $\mathrm{H}$ & -7.42191600 & -0.51936300 & 8.56937200 \\
\hline $\mathrm{H}$ & -5.60152500 & 0.42860500 & 7.22681400 \\
\hline $\mathrm{H}$ & -2.92842300 & -2.58844700 & 8.71928600 \\
\hline $\mathrm{H}$ & -2.05645300 & -0.91952200 & 7.27031600 \\
\hline $\mathrm{H}$ & -2.89798000 & 0.65127200 & 7.23310100 \\
\hline $\mathrm{H}$ & -3.9 & 1.38 & 65000 \\
\hline $\mathrm{H}$ & -2.61670200 & -2.56904300 & 5.61820900 \\
\hline $\mathrm{H}$ & -3.11239000 & -2.95972600 & 3.20752300 \\
\hline $\mathrm{H}$ & -4.51830400 & 1.11540700 & 2.96821800 \\
\hline $\mathrm{H}$ & -3.83195400 & -2.08188600 & 1.02583400 \\
\hline $\mathrm{H}$ & -4.79590800 & -1.39062200 & -0.94850100 \\
\hline $\mathrm{H}$ & & 2.20025600 & -12.02689600 \\
\hline $\mathrm{H}$ & -13.97042500 & -0.45 & -8.66810900 \\
\hline $\mathrm{H}$ & -11.60090600 & -1.07050300 & -8.85933500 \\
\hline $\mathrm{H}$ & -11.17454000 & 1.59337900 & -12.20383500 \\
\hline $\mathrm{H}$ & -9.25635700 & 0.44368700 & -11.43334300 \\
\hline $\mathrm{H}$ & -9.58692100 & -1.19535100 & -10.81049700 \\
\hline $\mathrm{H}$ & -8.39065200 & -1.75014000 & -9.05459800 \\
\hline $\mathrm{H}$ & -9.64882600 & 2.20284000 & -9.48725900 \\
\hline $\mathrm{H}$ & -8.76845700 & 2.80600700 & -7.23810000 \\
\hline
\end{tabular}




\begin{tabular}{|c|c|c|c|}
\hline H & -7.47124600 & -1.28343900 & -6.78300200 \\
\hline 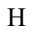 & -7.96625800 & 2.08694500 & -5.07034900 \\
\hline & -7.46764000 & 1.33836100 & -2.97784800 \\
\hline & -8.00971000 & -0.37222300 & 11.69555000 \\
\hline & -9.51170600 & -2.96680500 & 8.61614500 \\
\hline & -11.46222100 & -1.50082200 & 8.33110400 \\
\hline & -9.91136300 & 1.14055900 & 11.34487600 \\
\hline & -11.92160900 & 1.73593300 & 10.25124000 \\
\hline & -12.93762700 & 0.38860700 & 9.67786400 \\
\hline & -14.01215400 & 1.76313800 & 8.20553500 \\
\hline & -9.89087900 & 1.19145000 & 7.95347300 \\
\hline & -9.84029100 & 2.05571600 & 5.62219300 \\
\hline & -14.10019300 & 2.67951600 & 5.88836100 \\
\hline $\mathrm{H}$ & -11.02821900 & 2.75081100 & 3.61966200 \\
\hline $\mathrm{H}$ & -12.02551800 & 3.63487700 & 1.79455000 \\
\hline & -17.71238700 & 2.74109800 & -9.62738900 \\
\hline & -13.44234700 & 3.12209900 & -9.34173200 \\
\hline & -13.70646200 & 5.39043200 & -8.42460900 \\
\hline $\mathrm{H}$ & -17.97711500 & 5.00449000 & -8.69800700 \\
\hline $\mathrm{H}$ & -16.97683200 & 7.19032400 & 13800 \\
\hline $\mathrm{H}$ & -15.20401400 & 7.40256700 & 47300 \\
\hline I & -13.92529300 & 6.68843700 & -6.33925500 \\
\hline $\mathrm{H}$ & -18.09439300 & 6.60109400 & -6.26027100 \\
\hline $\mathrm{H}$ & -18.11153700 & 6.21617000 & -3.79972100 \\
\hline $\mathrm{H}$ & -13.79708600 & 30800 & 19400 \\
\hline H & -16.87560400 & 47400 & 8700 \\
\hline $\mathrm{H}$ & -15.70088500 & 5.27 & 46900 \\
\hline $\mathrm{C}$ & -12.08911000 & 1.61008000 & 91500 \\
\hline $\mathrm{C}$ & -13.31166900 & 2.25523900 & -5.29418600 \\
\hline $\mathrm{C}$ & -13.47578800 & 3.07798600 & -4.17634600 \\
\hline $\mathrm{C}$ & -12.42520400 & 3.27236000 & -3.27588700 \\
\hline $\mathrm{C}$ & -11.19790400 & 8100 & 72800 \\
\hline $\mathrm{C}$ & -11.04248700 & 9000 & 70600 \\
\hline $\mathrm{C}$ & -10.00613500 & 2.77329800 & 48300 \\
\hline $\mathrm{N}$ & -10.18471000 & 1300 & 14800 \\
\hline $\mathrm{O}$ & -8.86783200 & 2.36681100 & -2.97125500 \\
\hline $\mathrm{C}$ & -9.06569300 & 3.43757600 & -0.41905300 \\
\hline $\mathrm{C}$ & -9.43777500 & 2.70531500 & 0.86613500 \\
\hline $\mathrm{N}$ & -8.53794000 & 1.77 & 1500 \\
\hline $\mathrm{O}$ & -10.48878200 & 28900 & 10000 \\
\hline $\mathrm{N}$ & -8.84794700 & 0.99856200 & 1100 \\
\hline $\mathrm{C}$ & -7.97897700 & 0.10018000 & 2.72851700 \\
\hline $\mathrm{C}$ & -8.26018300 & -0.80360400 & 3.84836600 \\
\hline $\mathrm{C}$ & -9.52839300 & -0.84977200 & 4.45276100 \\
\hline $\mathrm{C}$ & -9.74755100 & -1.74179100 & 5.49298100 \\
\hline $\mathrm{N}$ & -8.7 & -2.5 & \\
\hline $\mathrm{C}$ & 56800 & -2.53022300 & 5.3 \\
\hline $\mathrm{C}$ & -7.26562500 & -1.66754300 & 4.32976900 \\
\hline $\mathrm{H}$ & -11.95301400 & 0.97186300 & -6.37059800 \\
\hline $\mathrm{H}$ & -14.12670300 & 2.12000700 & -5.99678400 \\
\hline $\mathrm{H}$ & -14.41944600 & 3.57960300 & -4.00290900 \\
\hline $\mathrm{H}$ & -12.58259200 & 8500 & -2.42829800 \\
\hline $\mathrm{H}$ & -10.10163000 & 1.28549300 & -4.75159000 \\
\hline $\mathrm{H}$ & -11.09733100 & 3.65553700 & -1.03342300 \\
\hline $\mathrm{H}$ & -8.87407900 & 4.48618700 & -0.16869200 \\
\hline $\mathrm{H}$ & -8.18717500 & 3.00261400 & -0.88600300 \\
\hline $\mathrm{H}$ & -7.70699200 & 1.54160400 & 0.68219500 \\
\hline 11 & -7.03628100 & -0.03928500 & 2.19989100 \\
\hline $\mathrm{H}$ & -10.32176300 & -0.20555700 & 4.10017400 \\
\hline & -10.71965400 & -1.79260600 & 5.96861700 \\
\hline & -6.81819200 & -3.20273900 & 5.76673300 \\
\hline & -6.27381600 & -1.66541000 & 3.89793300 \\
\hline
\end{tabular}

\section{Complex C2•7b -3}

$\begin{array}{cccc}\text { E }-5753.993798 \text { a.u. (zero imaginary freq.) } \\ \text { C } & -15.92780500 & 0.63846700 & -9.45461000 \\ \text { C } & -17.26829700 & -1.37622900 & -7.99092800 \\ \text { C } & -16.38817600 & -0.52574700 & -10.08616000 \\ \text { C } & -16.13449400 & 0.76555100 & -8.06949900 \\ \text { C } & -16.79801500 & -0.22484300 & -7.34615100 \\ \text { C } & -17.05038400 & -1.52140300 & -9.36435700 \\ \text { C } & -18.02288700 & -2.44021200 & -7.22550300 \\ \text { C } & -15.53044700 & -4.26800600 & -4.25430600 \\ \text { C } & -17.49496000 & -3.01191300 & -4.85545000 \\ \text { C } & -16.00678900 & -3.72490900 & -6.55296000 \\ \text { C } & -15.18826400 & -4.32321200 & -5.61678800 \\ \text { C } & -16.70751100 & -3.59335600 & -3.88529100 \\ \text { C } & -14.66527400 & -4.89866900 & -3.26278600 \\ \text { N } & -14.99843200 & -4.85720600 & -2.01243900 \\ \text { N } & -14.12365400 & -5.45559800 & -1.11844300\end{array}$

\begin{tabular}{|c|c|c|}
\hline-14.41566700 & -5.43506800 & 0.21166100 \\
\hline-7.80335700 & -3.73025100 & 9.98900100 \\
\hline-8.67306800 & -6.27978000 & 9.12782100 \\
\hline-8.65360700 & -4.51172000 & 10.78840900 \\
\hline-7.39388300 & -4.25301400 & 8.75329600 \\
\hline-7.81855400 & -5.51280600 & 8.32903300 \\
\hline-9.08503300 & -5.76926800 & 10.36478200 \\
\hline-9.17579800 & -7.62298100 & 8.64195300 \\
\hline-12.02533600 & -6.79846500 & 5.54888900 \\
\hline-9.85295600 & -7.57581700 & 6.23843500 \\
\hline-11.44999400 & -6.97916700 & 7.88150900 \\
\hline-12.37426700 & -6.67002500 & 6.90453000 \\
\hline-10.74199500 & -7.27590900 & 54500 \\
\hline-12.97966400 & -6.40327900 & 4.51829200 \\
\hline-12.64598600 & -6.49237200 & 3.27041200 \\
\hline-13.58048300 & -6.03527600 & 2.35227700 \\
\hline-13.29196800 & -6.07639200 & 1.02209700 \\
\hline-15.16078900 & 1.71904900 & -10.2 \\
\hline 03100 & 1.83489100 & 42900 \\
\hline-7.40188300 & -2.31112900 & 10.458 \\
\hline-6.90554500 & -2.38021000 & 11.937 \\
\hline-6.24794100 & -1.74875000 & 9.59084400 \\
\hline-4.09705800 & -0.73 & 8.05 \\
\hline 8200 & -2.36 & 9.61 \\
\hline 44100 & -0.61 & 600 \\
\hline-5.3 & -0.1 & 900 \\
\hline 550700 & -1.87 & 7200 \\
\hline-2.93867000 & -0.1762 & 7.25 \\
\hline-3.78 & -0.6 & 3.0 \\
\hline-3.6 & 0.7 & 100 \\
\hline & -1.5 & 5.16 \\
\hline-3.2 & -1.6 & 700 \\
\hline 7500 & 0.6 & 200 \\
\hline-4.11164700 & -0.82 & 1.66 \\
\hline-4.83380200 & 6500 & 8400 \\
\hline-5.08006000 & -0.19 & -0.29 \\
\hline-5.86822600 & 0.64977000 & -1.0 \\
\hline 193400 & 1.3 & 6800 \\
\hline-10 & 7100 & 4600 \\
\hline 54700 & 100 & 1800 \\
\hline-13.08233800 & 0.42670700 & -9.43410800 \\
\hline-11.72562000 & 0.10881200 & -9.50117900 \\
\hline-11.47059900 & 1.60931500 & -11.37072500 \\
\hline-9.42693700 & 0.37811000 & -10.53 \\
\hline 900 & 1.2 & -6.6 \\
\hline-8.1 & -0.28 & -8.4 \\
\hline-8.859 & 3900 & -8.73 \\
\hline-8.39062400 & 2.28175700 & -7.47268600 \\
\hline-7.70158000 & -0.01979300 & -7.208 \\
\hline-7.44876600 & 1.58317200 & -5.29 \\
\hline 2800 & 00 & -4.57 \\
\hline-6.6 & 000 & 1900 \\
\hline-5.91949800 & 8500 & -2.46 \\
\hline-8.63256900 & -1.37991100 & 10.34096300 \\
\hline-10.76457400 & 0.43936700 & 9.95918200 \\
\hline-8.73779600 & -0.21994400 & 11.12180100 \\
\hline-9.6 & -1.619 & \\
\hline-10 & -0.7 & 900 \\
\hline-9.7 & 0.68 & 10.92 \\
\hline 935700 & 57700 & 9.71670500 \\
\hline-11.81916900 & 2.95387900 & 5.69063800 \\
\hline-12.99847100 & 2.41022400 & 7.71866000 \\
\hline-10.66091500 & 2.06217200 & 7.60856400 \\
\hline & 200 & 2100 \\
\hline 2300 & 2.90068600 & 3000 \\
\hline-11.78021700 & 3.39959800 & 4.30222700 \\
\hline-12.84674300 & 3.90821400 & 3.76630300 \\
\hline-12.72947900 & 4.29213600 & 2.42707000 \\
\hline-13.82276600 & 4.81479700 & 1.81926900 \\
\hline 341400 & 3.09934600 & 70500 \\
\hline-15.72495800 & 5.65858800 & -8.42835200 \\
\hline 2156900 & 3.65226400 & -9.45611200 \\
\hline-14.25055700 & 3.84371900 & -9.08312300 \\
\hline-14.44213700 & 5.11073400 & -8.52540800 \\
\hline-16.81681200 & 4.91232400 & -8.89268800 \\
\hline-15.93382700 & 7.03090900 & -7.82121300 \\
\hline-15.83655800 & 6.52180000 & -3.56864100 \\
\hline-14.72893800 & 7.02100000 & -5.64617400 \\
\hline-17.07453500 & 6.69885000 & -5.62689900 \\
\hline-17.05868700 & 6.49893100 & -4.25927700 \\
\hline
\end{tabular}




\begin{tabular}{|c|c|c|c|}
\hline $\mathrm{C}$ & -14.66238000 & 6.81091600 & -4.28700500 \\
\hline $\mathrm{C}$ & -15.79649900 & 6.20325000 & -2.14282600 \\
\hline $\mathrm{N}$ & -14.65675500 & 5.92193100 & -1.59751800 \\
\hline $\mathrm{N}$ & -14.68014000 & 5.59858200 & -0.24592700 \\
\hline $\mathrm{C}$ & -13.55084400 & 5.14370000 & 0.35221600 \\
\hline $\mathrm{N}$ & -17.14475000 & -3.08487900 & -6.16947300 \\
\hline $\mathrm{O}$ & -15.43133400 & -4.95877100 & 0.74019800 \\
\hline $\mathrm{O}$ & -12.27144200 & -6.54439700 & 0.49589600 \\
\hline $\mathrm{N}$ & -10.20667500 & -7.41773100 & 7.54484000 \\
\hline $\mathrm{N}$ & -11.83282300 & 1.99066800 & 8.29217700 \\
\hline $\mathrm{N}$ & -15.92059200 & 6.94329100 & -6.30297800 \\
\hline $\mathrm{N}$ & -8.76829600 & 0.70177600 & -9.21117700 \\
\hline $\mathrm{N}$ & -3.19447700 & -0.31889400 & 5.76542600 \\
\hline $\mathrm{O}$ & -5.28704300 & -0.79234600 & -2.85143200 \\
\hline $\mathrm{O}$ & -6.47840200 & 1.64443800 & -0.56709000 \\
\hline $\mathrm{O}$ & -12.44329300 & 4.98706400 & -0.19226300 \\
\hline $\mathrm{O}$ & -14.94158800 & 5.01352300 & 2.32465900 \\
\hline $\mathrm{H}$ & -16.23296100 & -0.67024200 & -11.14725100 \\
\hline $\mathrm{H}$ & -15.77796000 & 1.64926900 & -7.55538400 \\
\hline $\mathrm{H}$ & -16.95300800 & -0.10167400 & -6.27946400 \\
\hline $\mathrm{H}$ & -17.40498200 & -2.41054800 & -9.87415200 \\
\hline $\mathrm{H}$ & -18.35936600 & -3.23847900 & -7.88938300 \\
\hline $\mathrm{H}$ & -18.88023500 & -2.02645300 & -6.69216300 \\
\hline $\mathrm{H}$ & -18.40911100 & -2.48729600 & -4.61963900 \\
\hline $\mathrm{H}$ & -15.78480000 & -3.73789500 & 11100 \\
\hline $\mathrm{H}$ & -14.29148800 & -4.83192600 & -5.94369200 \\
\hline $\mathrm{H}$ & -16.99950200 & -3.52714200 & -2.84774100 \\
\hline $\mathrm{H}$ & -13.76079100 & -5.38277200 & -3.62701700 \\
\hline $\mathrm{H}$ & -13.24619700 & -5.89175100 & -1.42763700 \\
\hline $\mathrm{H}$ & -8.99842400 & -4.13449800 & 7300 \\
\hline $\mathrm{H}$ & -6.74408600 & -3.67195600 & 54700 \\
\hline $\mathrm{H}$ & -7.49132700 & -5.89364100 & 09100 \\
\hline $\mathrm{H}$ & -9.74461300 & -6.35430500 & 10.99632700 \\
\hline $\mathrm{H}$ & -9.66688600 & -8.18006600 & 9.44079400 \\
\hline $\mathrm{H}$ & -8.37813600 & -8.22571000 & 8.20651700 \\
\hline $\mathrm{H}$ & -8.85454600 & -7.93781900 & 6.04144300 \\
\hline $\mathrm{H}$ & -11.66444100 & -6.87716500 & 3800 \\
\hline $\mathrm{H}$ & -13.35509700 & -6.31768000 & 62500 \\
\hline $\mathrm{H}$ & -10.44225500 & -7.39610200 & 93600 \\
\hline $\mathrm{H}$ & -13.94030100 & -6.01662400 & 4.85440900 \\
\hline $\mathrm{H}$ & -14.47135400 & -5.61858500 & 2.65032900 \\
\hline $\mathrm{H}$ & -15.33066900 & 2.70875200 & -12.20306400 \\
\hline $\mathrm{H}$ & -16.83 & 1.94901500 & -11.65101400 \\
\hline $\mathrm{H}$ & -15.50855100 & 0.94596600 & -12.28624800 \\
\hline $\mathrm{H}$ & -6.47470300 & -1.42309100 & 12.24258800 \\
\hline $\mathrm{H}$ & -6.13524200 & -3.14964500 & 12.03490600 \\
\hline $\mathrm{H}$ & -7.73215100 & -2.62407900 & 12.61002000 \\
\hline $\mathrm{H}$ & -4.82783800 & -3.24778600 & 10.22775600 \\
\hline $\mathrm{H}$ & -7.36864900 & -0.11995800 & 8.73472200 \\
\hline $\mathrm{H}$ & -5.50254000 & 0.77192300 & 7.41732000 \\
\hline $\mathrm{H}$ & -2.96025800 & -2.36486700 & 8400 \\
\hline $\mathrm{H}$ & -2.01244300 & -0.71061000 & 7.46935700 \\
\hline $\mathrm{H}$ & -2.79644000 & 0.88968300 & 7.44048300 \\
\hline $\mathrm{H}$ & -3.81364600 & 1.66720600 & 5.58906500 \\
\hline $\mathrm{H}$ & -2.59407800 & -2.31642900 & 5.78830700 \\
\hline $\mathrm{H}$ & -3.07198700 & -2.65914100 & 3.36 \\
\hline $\mathrm{H}$ & -4.37207500 & 1.45359200 & 3.17206700 \\
\hline $\mathrm{H}$ & -3.73495500 & -1.72774200 & 1.18819500 \\
\hline $\mathrm{H}$ & -4.65282500 & -0.98577500 & -0.78599600 \\
\hline $\mathrm{H}$ & -13.23512800 & 2.63035400 & -12.01392300 \\
\hline $\mathrm{H}$ & -13.69616700 & -0.03875100 & -8.67511800 \\
\hline $\mathrm{H}$ & -11.30606600 & -0.59995700 & -8.79507700 \\
\hline $\mathrm{H}$ & -10.84 & 2.08017400 & -12.12208200 \\
\hline $\mathrm{H}$ & -8.92872700 & 0.97339100 & -11.30456500 \\
\hline $\mathrm{H}$ & -9.24424600 & -0.68193200 & -10.72081400 \\
\hline $\mathrm{H}$ & -8.15067000 & -1.26938000 & -8.91147100 \\
\hline $\mathrm{H}$ & -9.33042700 & 2.70496300 & -9.37081300 \\
\hline $\mathrm{H}$ & -8.48952600 & 3.29294600 & -7.10235400 \\
\hline $\mathrm{H}$ & -7.26775800 & -0.81693900 & -6.62276100 \\
\hline $\mathrm{H}$ & -7.72810500 & 2.56011900 & -4.91917600 \\
\hline $\mathrm{H}$ & -7.25860600 & 1.79788500 & -2.82132200 \\
\hline $\mathrm{H}$ & -7.98896000 & 0.00702400 & 11.86905000 \\
\hline $\mathrm{H}$ & -9.53851300 & -2.47651400 & 8.72080200 \\
\hline $\mathrm{H}$ & -11.43049900 & -0.93763800 & 8.43678000 \\
\hline $\mathrm{H}$ & -9.82867400 & 1.59302500 & 11.51811600 \\
\hline $\mathrm{H}$ & -11.77979300 & 2.29476300 & 10.38974800 \\
\hline $\mathrm{H}$ & -12.85783200 & 0.99036200 & 9.83019800 \\
\hline $\mathrm{H}$ & -13.89379200 & 2.32375000 & 8.31715400 \\
\hline $\mathrm{H}$ & -9.77323700 & 1.72278900 & 8.11942200 \\
\hline $\mathrm{H}$ & -9.68945600 & 2.56611400 & 5.78112200 \\
\hline
\end{tabular}

\begin{tabular}{|c|c|c|c|}
\hline & -13.94799500 & 3.21795300 & 5.99069800 \\
\hline $\mathrm{H}$ & -10.85137600 & 3.25918500 & 3.75741700 \\
\hline $\mathrm{H}$ & -11.83642700 & 4.13227700 & 1.91312600 \\
\hline & -17.48131500 & 3.09398600 & -9.80659700 \\
\hline & -13.24868800 & 3.44360500 & -9.15255100 \\
\hline & -13.58496100 & 5.67320900 & -8.17122500 \\
\hline & -17.81837900 & 5.32228300 & -8.82200300 \\
\hline & -16.89871300 & 7.45180500 & -8.10478000 \\
\hline & -15.13387000 & 7.71974600 & 1200 \\
\hline & -13.85640900 & 7.24541700 & -6.24205800 \\
\hline & -17.98476300 & 6.65862200 & -6.20726600 \\
\hline & -17.98687300 & 6.30028300 & -3.74093800 \\
\hline & -13.70876200 & 6.86581200 & -3.78384900 \\
\hline & -16.74234200 & 6.18487100 & -1.60389300 \\
\hline & -15.5 & 00 & 1200 \\
\hline & -11.8 & 00 & 00 \\
\hline & -13.0 & 1800 & 4700 \\
\hline & -13.22743400 & 3.57913600 & -4.08967200 \\
\hline & -12.18908000 & 3.76703900 & 0800 \\
\hline & -10.9 & 3.10 & 800 \\
\hline & -10.8 & 2.27 & 000 \\
\hline & -9.78 & 325 & -2.4 \\
\hline $\mathrm{N}$ & -9.97 & 00 & 00 \\
\hline & -8.64 & 00 & -2.82 \\
\hline & -8.85 & 00 & -0.277 \\
\hline C & -9.25101 & 3.19 & 1.00 \\
\hline $\mathrm{N}$ & -8.37 & 2.23 & 1.40 \\
\hline & -10.3 & 3.4 & 300 \\
\hline $\mathrm{N}$ & -8.70 & 1.47 & 2.5 \\
\hline & -7.86 & 0.55 & 5400 \\
\hline & -8.18278700 & -0.3416 & 3.994 \\
\hline & -9.45910000 & -0.3524 & 4.58 \\
\hline & -9.71754900 & -1.24112500 & 5.61766100 \\
\hline & & & 6.09 \\
\hline C & -7.56 & -2.09 & 5.52 \\
\hline $\mathrm{C}$ & -7.22 & -1.23 & 4.48 \\
\hline & -11.6 & 8000 & -6.25079200 \\
\hline $\mathrm{H}$ & -13.86020800 & 2.62199000 & -5.91643800 \\
\hline & -14.16800100 & 4.09095100 & -3.93307900 \\
\hline & -12.35332000 & 4.42421700 & -2.33024200 \\
\hline & & 1.75 & -4.60 \\
\hline $\mathrm{H}$ & -10.8 & 4.12 & 7000 \\
\hline $\mathrm{H}$ & -8.66180300 & 4.96456500 & -0.02827300 \\
\hline $\mathrm{H}$ & -7.98044400 & 3.47304700 & -0.73533600 \\
\hline $\mathrm{H}$ & -7.53883400 & 1.99623600 & 0.83643600 \\
\hline $\mathrm{H}$ & -6.91827100 & 0.39488300 & 2.36181600 \\
\hline & -10.22882400 & 0.31581700 & 4.22358700 \\
\hline $\mathrm{H}$ & -10.69665200 & -1.26551900 & 6.08068400 \\
\hline 11 & -6.83626400 & -2.78900200 & 5.92169900 \\
\hline $\mathrm{H}$ & -6.22311600 & -1.26132800 & 4.0677760 \\
\hline
\end{tabular}


Table S2. The solubility of Products with or without the Cages in Water at $25{ }^{\circ} \mathrm{C}$

\begin{tabular}{ccccc}
\hline Entry & product & $\begin{array}{c}\text { Solubility } \\
(\mathrm{mM})^{a}\end{array}$ & $\begin{array}{c}\text { Solubility } \\
(\mathrm{mM})^{b}\end{array}$ & $\begin{array}{c}\text { Solubility } \\
(\mathrm{mM})^{c}\end{array}$ \\
\hline 1 & $\mathbf{7 a}$ & 0.21 & 1.1 & 1.2 \\
2 & $\mathbf{7 b}$ & 1.35 & 1.2 & 1.2 \\
3 & $\mathbf{9 a}$ & 0.026 & 1.2 & 1.1 \\
4 & $\mathbf{9 b}$ & 0.81 & 1.1 & 1.2 \\
5 & $\mathbf{9 c}$ & 0.52 & 1.2 & 1.2 \\
6 & $\mathbf{9 d}$ & 1.05 & 1.2 & 1.1 \\
7 & $\mathbf{9 e}$ & 0.008 & 1.1 & 1.2 \\
8 & $\mathbf{9 f}$ & 0.002 & 1.2 & 1.2 \\
9 & $\mathbf{9 g}$ & 0.003 & 1.1 & 1.1 \\
10 & $\mathbf{9 h}$ & - & 1.1 & 1.1 \\
11 & $\mathbf{9 i}$ & - & 1.2 & 1.2 \\
12 & $\mathbf{9 j}$ & - & 1.2 & 1.2 \\
13 & $\mathbf{9 k}$ & 0.004 & 1.2 & \\
\hline${ }^{a}$ Without the cages, ${ }^{\mathrm{b}}[\mathbf{C 1}]=1.25 \mathrm{mM}^{2}$ and $^{\mathrm{c}}[\mathbf{C 2}]=1.25 \mathrm{mM}$, determined by HPLC \\
using anisole as the internal standard.
\end{tabular}

